Argonne

\title{
Annual Report of Monitoring at Morrill, Kansas, in 2017
}

\author{
Environmental Science Division
}




\begin{abstract}
About Argonne National Laboratory
Argonne is a U.S. Department of Energy laboratory managed by UChicago Argonne, LLC under contract DE-AC02-06CH11357. The Laboratory's main facility is outside Chicago, at 9700 South Cass Avenue, Argonne, Illinois 60439. For information about Argonne and its pioneering science and technology programs, see www.anl.gov.
\end{abstract}

\title{
DOCUMENT AVAILABILITY
}

Online Access: U.S. Department of Energy (DOE) reports produced after 1991 and a growing number of pre-1991 documents are available free via DOE's SciTech Connect (http://www.osti.gov/scitech/)

Reports not in digital format may be purchased by the public from the National Technical Information Service (NTIS):

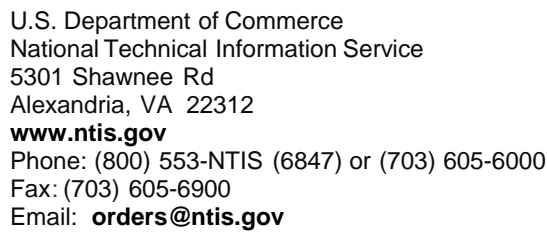

Reports not in digital format are available to DOE and DOE contractors from the Office of Scientific and Technical Information (OSTI):

U.S. Department of Energy

Office of Scientific and Technical Information

P.O. Box 62

Oak Ridge, TN 37831-0062

www.osti.gov

Phone: (865) 576-8401

Fax: (865) 576-5728

Email: reports@osti.gov

\section{Disclaimer}

This report was prepared as an account of work sponsored by an agency of the United States Government. Reference herein to any specific commercial product, process, or service by trade name, trademark, manufacturer, or otherwise, does not necessarily constitute or imply its endorsement, recommendation, or favoring by the United States Government or any agency thereof. The views and opinions of document authors expressed herein do not necessarily state or reflect those of the United States Government or any agency thereof, Argonne National Laboratory, or UChicago Argonne, LLC. 


\section{Annual Report of Monitoring at Morrill, Kansas, in 2017}

by

Applied Geosciences and Environmental Management Section Environmental Science Division, Argonne National Laboratory

August 2018

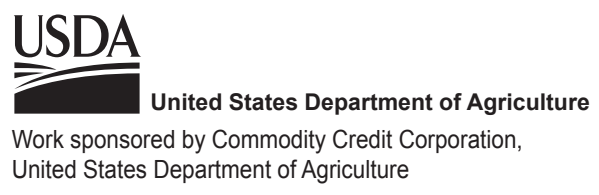




\section{Contents}

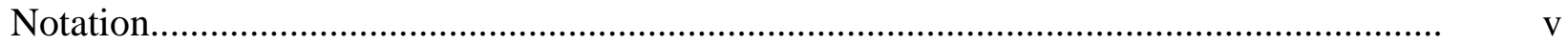

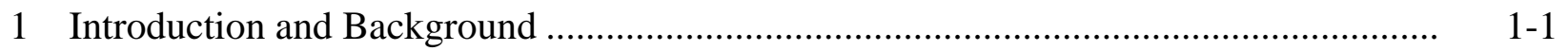

2 Sample Collection and Analysis Activities............................................................ $2-1$

2.1 Measurement of Groundwater Levels........................................................... 2-1

2.2 Monitoring Well and Private Well Sampling and Analyses ................................ 2-1

2.3 Surface Water and Sediment Sampling and Analyses........................................ 2-3

2.4 Vegetation Sampling and Analyses ................................................................ 2-3

2.5 Evaluation of Private Wells ........................................................................... 2-4

2.6 Handling and Disposal of Investigation-Derived Waste ................................... 2-4

2.7 Quality Control for Sample Collection, Handling, and Analysis ........................ 2 2-4

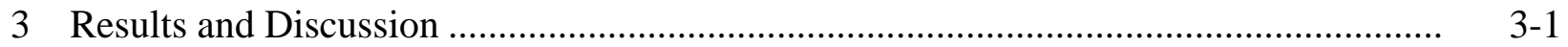

3.1 Groundwater Level Data...........................................................................

3.2 Groundwater Analysis Results..................................................................... $3-2$

3.3 Surface Water and Sediment Analysis Results ................................................ 3-2

3.4 Private Well Evaluation Results ......................................................................... 3-3

4 Conclusions, Future Activities, and Recommendations ........................................

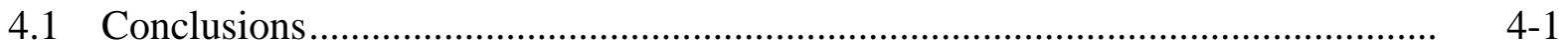

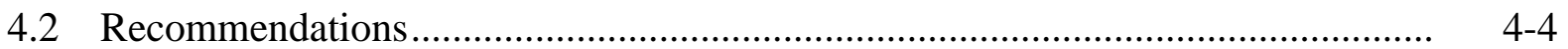

5 References .............................................................................................

Appendix A: $\quad$ Sampling Activities at Morrill in 2017................................................. A-1

Appendix B: Results from the AGEM Laboratory and TestAmerica for Quality

Control Samples Analyzed in 2017 ........................................................ B- B-1

Supplement 1: Sample Documentation from TestAmerica Laboratories, Inc., for Groundwater Verification Samples (on CD) ........................................... S1-1 


\section{Figures}

1.1 Location of Brown County and Morrill, Kansas ................................................... 1-5

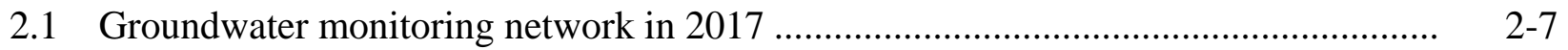

2.2 Locations of surface water and creek bed sediment sampling along Terrapin Creek in 2016

3.1 Potentiometric surface based on water levels measured manually on May 30-June 2, 2017

3.2 Potentiometric surface based on water levels measured manually on December 19, 2017

3.3 Interpretive south-north geologic section illustrating the stratigraphic relationships among screened intervals of wells near Terrapin Creek

3.4 Hydrographs summarizing results of long-term water level monitoring in 2017.

3.5 Carbon tetrachloride concentrations in groundwater, May 2016 and May-June 2017.

4.1 Historical carbon tetrachloride concentrations in groundwater, 1995-2017.

\section{Tables}

3.1 Hand-measured groundwater levels in January 2015-December 2017

3.2 Results of analyses at the AGEM Laboratory for VOCs in groundwater samples collected in 2003-2017.

3.3 Field measurements for groundwater samples collected in 2003-2017

3.4 Results of analyses at the AGEM Laboratory for VOCs in surface water and sediment samples collected in 2007-2016

3.5 Evaluation results for private wells in 2017

A.1 Sequence of sampling activities at Morrill, Kansas, in 2017.

B.1 Analytical results from the AGEM Laboratory for quality control samples collected to monitor sample collection and handling activities in 2017. 
B.2 Analytical results from the AGEM Laboratory for dual analyses of samples collected in 2017

B.3 Results for groundwater samples collected in 2017 and submitted for verification organic analyses..... 


\section{Notation}

AGEM Applied Geosciences and Environmental Management

AMSL above mean sea level

BGL below ground level

${ }^{\circ} \mathrm{C} \quad$ degree(s) Celsius

CCC Commodity Credit Corporation

CD compact disc

DO dissolved oxygen

EPA U.S. Environmental Protection Agency

$\mathrm{ft} \quad$ foot (feet)

gal gallon(s)

IGA Intergovernmental Agreement

in. inch(es)

KDHE Kansas Department of Health and Environment

L liter(s)

$\mu \mathrm{g} / \mathrm{kg} \quad$ microgram(s) per kilogram

$\mu \mathrm{g} / \mathrm{L} \quad$ microgram(s) per liter

$\mu \mathrm{S} / \mathrm{cm} \quad$ microsiemen(s) per centimeter

MCL maximum contaminant level

$\mathrm{mg} / \mathrm{L} \quad$ milligram(s) per liter

mi mile(s)

min minute(s)

$\mathrm{mV} \quad$ millivolt(s)

ORP oxidation-reduction potential

RBSL risk-based screening level

RMP Risk Management Program

SOP standard operating procedure

TOC top of casing

USDA U.S. Department of Agriculture

VOC volatile organic compound 


\section{Annual Report of Monitoring at Morrill, Kansas, in 2017}

\section{Introduction and Background}

Carbon tetrachloride contamination in groundwater at Morrill, Kansas, was initially identified in 1985 during statewide testing of public water supply wells for volatile organic compounds (VOCs). High levels of nitrate were also present in the public wells. The city of Morrill is located in Brown County in the northeastern corner of the state, about 7 mi east of Sabetha (Figure 1.1). The population of Morrill as of the 2010 Census was approximately 230 (down from 277 in 2000). All residents of Morrill now obtain their drinking water from the Sabetha municipal water system via a pipeline constructed in 1991.

From 1922 to 1991, eight different public water supply wells served the Morrill municipal system. Because of poor water quality, use of the public wells was discontinued over time. The water quality problems included high nitrate levels attributed to numerous animal feeding operations in the vicinity and application of fertilizer to surrounding agricultural lands, as well as naturally occurring excessive hardness and elevated iron, sulfate, and total dissolved solids concentrations above acceptable levels for potable water supply. At the recommendation of the Kansas Department of Health and Environment (KDHE 1989), use of local groundwater by the city of Morrill as a municipal water supply was terminated in 1991 because of the high nitrate levels and poor water quality. Public water was then obtained from the Sabetha municipal water system.

Investigations of the carbon tetrachloride and nitrate contamination by the KDHE in 1989, 1994, and 1996 (KDHE 1989; GeoCore 1994a-d, 1996) identified a localized plume of carbon tetrachloride in groundwater extending downgradient from a grain storage facility in the northwestern section of Morrill. No localized source of nitrate contamination was identified. The identified grain storage facility was operated by the Commodity Credit Corporation of the U.S. Department of Agriculture (CCC/USDA) from 1950 to 1971. After termination of the CCC/USDA grain storage operations in 1971, the property and existing grain bins continued to be used for private grain storage until the present time. Prior to 1986, commercial grain fumigants containing carbon tetrachloride were commonly used by the CCC/USDA and by private and commercial grain storage operations to preserve grain. 
Because the identified carbon tetrachloride contamination in groundwater could potentially be linked, in part, to historical use of carbon tetrachloride-based fumigants at its former facility, in 2003 the CCC/USDA assumed responsibility for investigation of the carbon tetrachloride contamination. The CCC/USDA involvement began with the development and implementation of a work plan for a Phase I expedited site characterization (Argonne 2003). That investigation and subsequent investigations (Argonne 2004, 2005a) were performed by the Environmental Science Division of Argonne National Laboratory.

The initial investigation by the CCC/USDA in 2003 determined that soils at the former facility were not impacted by grain fumigation activities. Neither carbon tetrachloride nor chloroform (the primary degradation product of carbon tetrachloride) was detected in near-surface soils or in subsurface soils collected to bedrock. Because no identifiable human health risk was associated with either carbon tetrachloride or chloroform in shallow soils, the conclusion was that no further threat of contamination to groundwater was present.

In September 2005, the CCC/USDA initiated periodic sampling of groundwater, in accord with a program (Argonne 2005b) approved by the KDHE (2005), to monitor carbon tetrachloride concentrations in the groundwater.

Under the KDHE-approved monitoring plan (Argonne 2005b), groundwater was sampled twice yearly for VOCs analyses through 2011. During the initial two years of monitoring, analysis for selected geochemical parameters was also conducted to aid in the evaluation of possible natural contaminant degradation (reductive dechlorination) processes in the subsurface environment. Consistently low levels of dissolved oxygen (DO) and oxidation-reduction potential (ORP) at monitoring well MW1D (in the deepest portion of the contaminated aquifer) and the presence of chloroform (the primary degradation product of carbon tetrachloride) suggested that some degree of reductive dechlorination was occurring.

The maximum carbon tetrachloride concentration reported in groundwater at the site was $390 \mu \mathrm{g} / \mathrm{L}$, in a sample collected from monitoring well MW3S on the former CCC/USDA property in 1995 (GeoCore 1996). The concentrations have declined significantly during long-term monitoring conducted initially by the KDHE and currently by the CCC/USDA. The present maximum levels of $<50 \mu \mathrm{g} / \mathrm{L}$ in groundwater confirm that no continuing soil source remains at the former CCC/USDA facility. Nevertheless, carbon tetrachloride concentrations exceeding the KDHE Tier 2 risk-based screening level (RBSL) of $5.0 \mu \mathrm{g} / \mathrm{L}$ are still present in some wells. 
The analytical results for groundwater sampling events at Morrill in 2005-2016 were documented previously (Argonne 2006, 2007a,b, 2008a,b, 2009, 2010, 2011, 2012, 2013, 2014, 2015, 2016, 2017). Those results consistently demonstrated the presence of carbon tetrachloride contamination, at concentrations exceeding the RBSL of $5.0 \mu \mathrm{g} / \mathrm{L}$, in a groundwater plume extending southward from the former CCC/USDA facility, toward Terrapin Creek at the southern edge of the town.

Terrapin Creek is identified by the KDHE (2001) as tributary segment 308 to Walnut Creek, which in turn is located in the Big Nemaha Subbasin of the Missouri Basin Walnut Creek. Terrapin Creek is classified by the KDHE (2001) as impaired because of high levels of fecal coliform bacteria. Prevention of further degradation of Terrapin Creek by carbon tetrachloride is the regulatory driver for ongoing monitoring of the carbon tetrachloride plume (KDHE 2007). No trend of increasing carbon tetrachloride concentrations near the creek has been indicated.

In 2006, the CCC/USDA recommended expansion of the approved monitoring program to include the collection and analysis of surface water samples along Terrapin Creek (Argonne 2007a). At the request of the KDHE (2007), locations for both surface water and shallow sediment sampling were discussed with the KDHE in January 2007. An addendum to the existing monitoring plan (dated May 2007; in Appendix A, Argonne 2010) and a standard operating procedure (SOP) for sediment sampling (SOP AGEM-15; in Appendix B, Argonne 2010) were submitted to the KDHE on the basis of these discussions and were subsequently approved (KDHE 2008a,b). To supplement the original scope of the monitoring, in 2007-2011 Argonne also sampled natural vegetation at locations in the contaminant plume and along Terrapin Creek for VOCs analyses.

In August 2010, indoor air sampling was conducted at seven residences, one church, and one business overlying the contaminant plume to evaluate the potential for vapor intrusion. Carbon tetrachloride contamination was not detected.

The KDHE (2012) subsequently approved an annual monitoring schedule and also approved discontinuation of vegetation sampling. Sampling of the Isch and Grimm irrigation (TD12) wells was discontinued in 2016 per KDHE (2015) approval. The KDHE (2016) also approved discontinuation of sampling at monitoring wells MW2S, MW4S and MW10S and a reduction in the frequency of surface water and sediment sampling to every other year. Most recently, the KDHE (2017) approved discontinuation of sample collection at MW1S using the low- 
flow method and passive diffusion bag samplers; samples at MW1S will continue to be collected using only the three-well-volume purging method.

The May-June 2017 groundwater and surface water and sediment sampling events reported here were conducted in accord with the monitoring plan (Argonne 2005b) and the addendum to that plan (Appendix A in Argonne 2010). Groundwater sampling occurred in a network of nine monitoring wells and two private wells, at locations approved by the KDHE (2008a, 2015, 2016). The current report presents the data generated in 2017. 


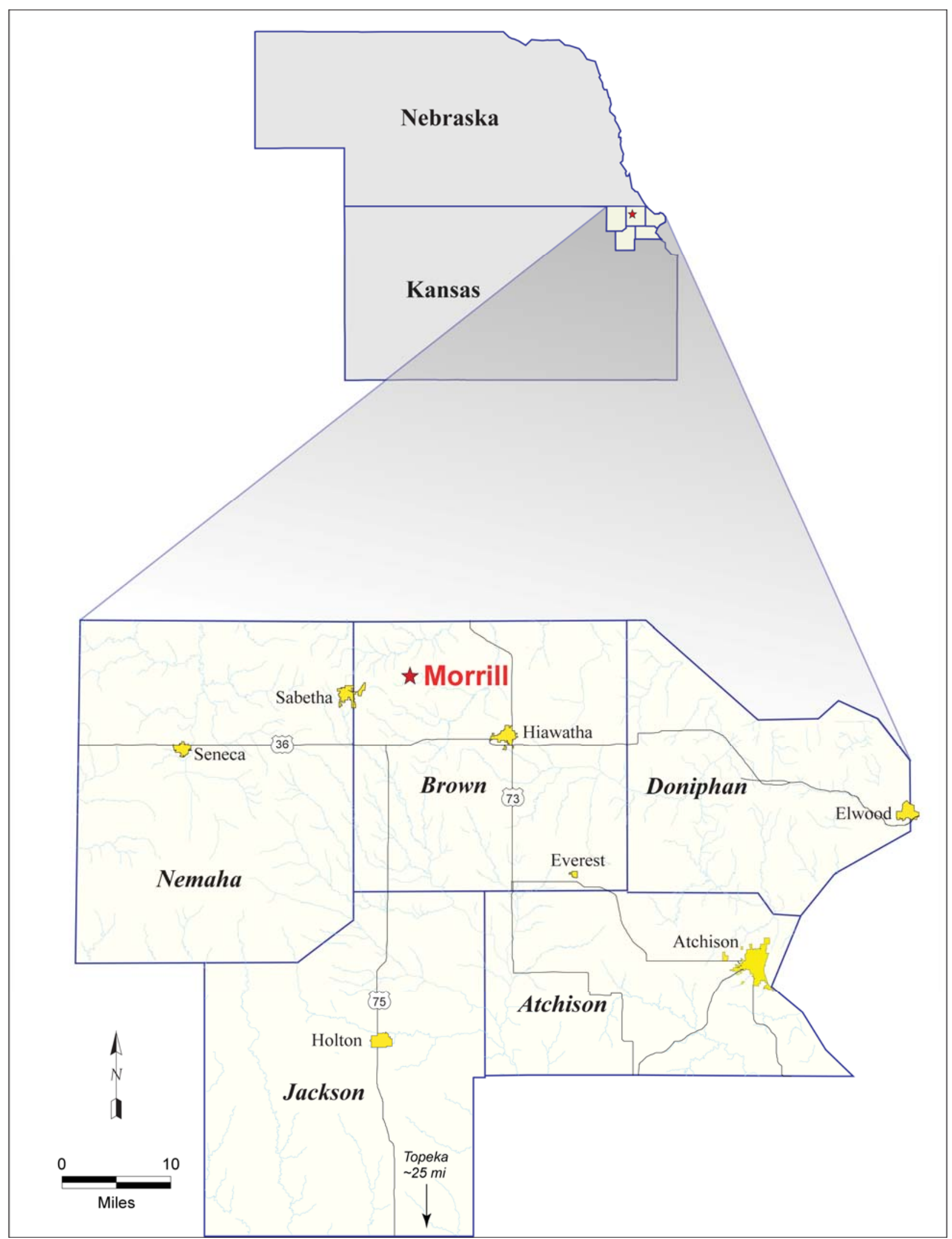

FIGURE 1.1 Location of Brown County and Morrill, Kansas. 


\section{Sample Collection and Analysis Activities}

\subsection{Measurement of Groundwater Levels}

Data recorders have been installed in wells MW1D, MW1S-MW4S, MW6S-MW8S, and MW11S to gather long-term data on the groundwater elevation and gradient at Morrill, in order to evaluate daily to seasonal variations. Recorders were installed in wells MW1D and MW11S in February 2013 to generate additional information on the apparent hydraulic impact of seasonal pumping of the Grimm irrigation well (TD12 location in Figures 3.6 and 4.1). This effect has been noted previously (Argonne 2012, 2013, 2014, 2015, 2016, 2017). To confirm the long-term data and to define the potentiometric surface, depths to groundwater and total well depths from the tops of the well casings are measured periodically in all monitoring wells in conjunction with the data recorder downloads, as well as during each groundwater sampling event, with an accuracy of $\pm 0.01 \mathrm{ft}$.

To document the current reporting period, data recorders were downloaded and water levels were measured manually on December 19, 2017. Data recorders were also downloaded and water levels measured manually in conjunction with the sampling event on May 30-June 2, 2017.

The groundwater level data are presented and discussed in Section 3.1.

\subsection{Monitoring Well and Private Well Sampling and Analyses}

Monitoring wells MW1D, MW1S, MW3S, MW5S-MW9S, and MW11S and the Stone and Rilinger private wells (Figure 2.1) were sampled on May 31-June 2, 2017. Sampling of the Isch and Grimm irrigation (TD12) wells was discontinued in 2016 per KDHE (2015) approval, and sampling of monitoring wells MW2S, MW4S and MW10S was discontinued in 2017 per KDHE (2016) approval.

Low-flow samples were collected from monitoring wells using a bladder pump. After measurement of water levels, each monitoring point was purged of a small volume, in accord with U.S. Environmental Protection Agency (EPA) procedure EPA/540/S-95/504 (Puls and 
Barcelona 1996; Yeskis and Zavala 2002) and the equipment manufacturers' instructions. Field measurements of temperature, $\mathrm{pH}$, conductivity, $\mathrm{DO}$, and ORP were taken during purging until the measurements stabilized. Field measurements of iron(II) were made as outlined in the monitoring plan (Argonne 2005b), in accord with procedures in the Master Work Plan (Argonne 2002).

Samples from the Rilinger private well was collected after a 5-min purge with a dedicated pump. The sample from the Stone private well was collected after purging of the well by bailing.

Prior sampling at well MW1S, which is located near the center of the contaminant plume and is screened over a 40 -ft interval, has indicated that a representative sample is not collected by the low-flow sampling procedure. Therefore, beginning in 2008 well MW1S was also sampled periodically after purging of three well volumes with a Redi-Flo pump. In addition, in 2013 and 2014 the well was sampled with passive diffusion bag samplers, placed at 2-ft intervals (20-52 ft below ground level [BGL]). The samplers were placed in the well on April 2, 2012, and subsequently retrieved for sampling on an annual basis in 2013, 2014, 2015, and 2016. This comparison sampling was discontinued in 2017 per KDHE (2017) approval, after which MW1S was sampled only after purging of three well volumes with a Redi-Flo pump in 2017.

The sequence of activities during the 2017 sampling event is summarized in Appendix A, Table A.1.

Groundwater samples for VOCs analyses were collected in appropriate laboratory containers, labeled, packaged, and chilled to $4^{\circ} \mathrm{C}$ by placement in ice-filled coolers. The samples were shipped overnight to the Applied Geosciences and Environmental Management (AGEM) Laboratory at Argonne for VOCs analyses with EPA Method 524.2 (EPA 1995). Separate aliquots of selected samples (chosen in the field) were shipped to TestAmerica Laboratories, Inc., South Burlington, Vermont, for verification VOCs analyses.

The results of the groundwater VOCs analyses are presented and discussed in Section 3.2. 


\subsection{Surface Water and Sediment Sampling and Analyses}

At the direction of the KDHE (2007), surface water samples and corresponding samples of the underlying shallow sediments in the creek bed are collected for VOCs analyses in conjunction with compliance monitoring events at five locations along Terrapin Creek (Figure 2.2), as outlined in the monitoring plan addendum (Appendix A in Argonne 2010). The KDHE (2016) approved a reduction in the frequency of surface water and sediment sampling; beginning in 2017, surface water and sediment sampling will be conducted every other year, rather than in conjunction with every annual monitoring event. The next surface water and sediment sampling event will be conducted in 2018.

The sampling is conducted in accord with procedures in the Master Work Plan (Argonne 2002) and SOP AGEM-15 (Appendix B in Argonne 2010). Surface water flow in Terrapin Creek south of Morrill originates at the outfall from an earthen dam and retention pond approximately 1,900 ft southwest of the former CCC/USDA facility (Figure 2.2). Surface water and sediment sampling location SMB, which is directly downstream from this outfall, is believed to lie upgradient, or cross-gradient, to groundwater flow (and possible contaminant migration) from the vicinity of the former CCC/USDA facility (see Section 3.1). Sampling locations SM1-SM4 lie downgradient and downstream from the carbon tetrachloride detections identified previously at MW8S and elsewhere in the monitoring well network.

The results of past surface water and sediment VOCs analyses are presented and discussed in Section 3.3.

\subsection{Vegetation Sampling and Analyses}

In 2007-2011, vegetation samples were collected at locations in the contaminant plume and along Terrapin Creek and its tributaries south and southwest of the former CCC/USDA facility. The locations were selected along the direction of groundwater flow from the former facility. Vegetation samples were collected at 18 locations in July 2007 (Argonne 2008a), at 25 locations in July 2008 (Argonne 2009), and at 22 locations in August 2009 (Argonne 2010). In July 2010 and July 2011, branch tissue samples were collected at 42 locations from mature ash, cottonwood, elm, hackberry, juniper, maple, mulberry, oak, Osage orange, pear, pine, walnut, and willow trees (Argonne 2011, 2012). 
Sporadic detections of carbon tetrachloride at trace levels in vegetation samples over time confirmed uptake of carbon tetrachloride in the tree tissues, but declining concentrations in the groundwater precluded identification of a trend in the vegetation results. With the approval of the KDHE (2012), sampling of native vegetation has been terminated as part of the overall monitoring program.

\subsection{Evaluation of Private Wells}

At the request of the KDHE (2014), Argonne began conducting an evaluation of the use, condition, and ownership of private wells for the 2014 reporting period. Evaluation of the private wells was performed for the 2017 monitoring period on February 27, 2018. This evaluation included the Allen, Edwards (formerly Manning), Rilinger, and Stone private wells. The Argonne representative conducting the evaluations also spoke with the current owner (Gruber) of the former Manning/Cain property on which the home was razed after a fire in 2015 and on which no visible sign of any well is present. Evaluation of the Isch and Grimm irrigation (TD12) wells was discontinued per KDHE (2015) approval.

The results of the private well evaluation are presented and discussed in Section 3.5.

\subsection{Handling and Disposal of Investigation-Derived Waste}

Purge water generated as potentially contaminated investigation-derived waste during the May-June 2017 annual monitoring was containerized on-site in 55-gal drums. A composite wastewater sample was not collected during the 2017 sampling event; the purge water remains containerized on-site. A composite sample will be collected for laboratory analysis during the next sampling event in 2018, and the analytical results will be submitted to the KDHE in conjunction with a request for approval to dispose of the accumulated wastewater.

\subsection{Quality Control for Sample Collection, Handling, and Analysis}

The quality control/quality assurance procedures followed during the 2017 monitoring events are described in detail in the Master Work Plan (Argonne 2002) and SOP AGEM-15 (Appendix B in Argonne 2010). These procedures are summarized as follows: 
- Sample collection and handling activities were monitored by the documentation of samples as they were collected and the use of chain-of-custody forms and custody seals to ensure sample integrity during handling and shipment.

- Samples designated for VOCs analyses were received with custody seals intact and at the appropriate preservation temperature. All samples were analyzed within the required holding times.

- Quality control samples (one field blank, three equipment rinsates, and two trip blanks) collected to monitor sample collection and handling activities were free of VOC contamination. Method blanks used to monitor analytical methodologies were free of carbon tetrachloride and chloroform contamination. Analytical results for quality control samples collected to monitor samplehandling activities are in Appendix B, Table B.1.

- Groundwater samples were analyzed for VOCs at the AGEM Laboratory by the purge-and-trap method on a gas chromatograph-mass spectrometer system. Calibration checks analyzed with each sample delivery group were required to be within $\pm 20 \%$ of the standard. Surrogate standard determinations performed on samples and blanks were within the specified range of $80-120 \%$ for all samples, in either the initial analysis or a successful reanalysis.

- Accuracy and precision of the analytical methodology were evident in the analysis of one replicate sample and duplicate analysis of one additional sample. The average relative percent difference value was approximately $4 \%$ between the initial analysis and the associated quality control analysis for carbon tetrachloride concentrations present above the method detection limit (Appendix B, Table B.2). The quality control range for relative percent difference between dual analyses is $\pm 20 \%$. The groundwater analytical data from the AGEM Laboratory are acceptable for quantitative determination of contaminant distribution.

- In accordance with the procedures defined in the Master Work Plan (Argonne 2002), the analyses of water samples at the AGEM Laboratory were verified by a second laboratory. Accordingly, groundwater samples collected during the 
2017 monitoring event were submitted to TestAmerica for analysis according to the EPA's Contract Laboratory Program methodology. Complete analytical results for one groundwater sample (from MW5S) and one trip blank collected in May-June 2017 are in sample delivery group 200-38792 in Supplement 1 (on compact disc [CD]). (Note: samples were inadvertently collected and shipped to TestAmerica from two additional wells, MW4S and MW10S, which had previously been eliminated from the monitoring network per the KDHE [2016]. Results for these samples are included in sample delivery group 200-38792 but are not summarized in this report.) The results are listed in Appendix B, Table B.3. Agreement in the results from the two laboratories is good; neither laboratory detected carbon tetrachloride or chloroform in either sample above the respective method detection limits, with the exception of a trace detection of chloroform in the trip blank analyzed at TestAmerica. The concentrations detected in groundwater in analyses at the AGEM Laboratory are supported by the verification analyses at TestAmerica. 


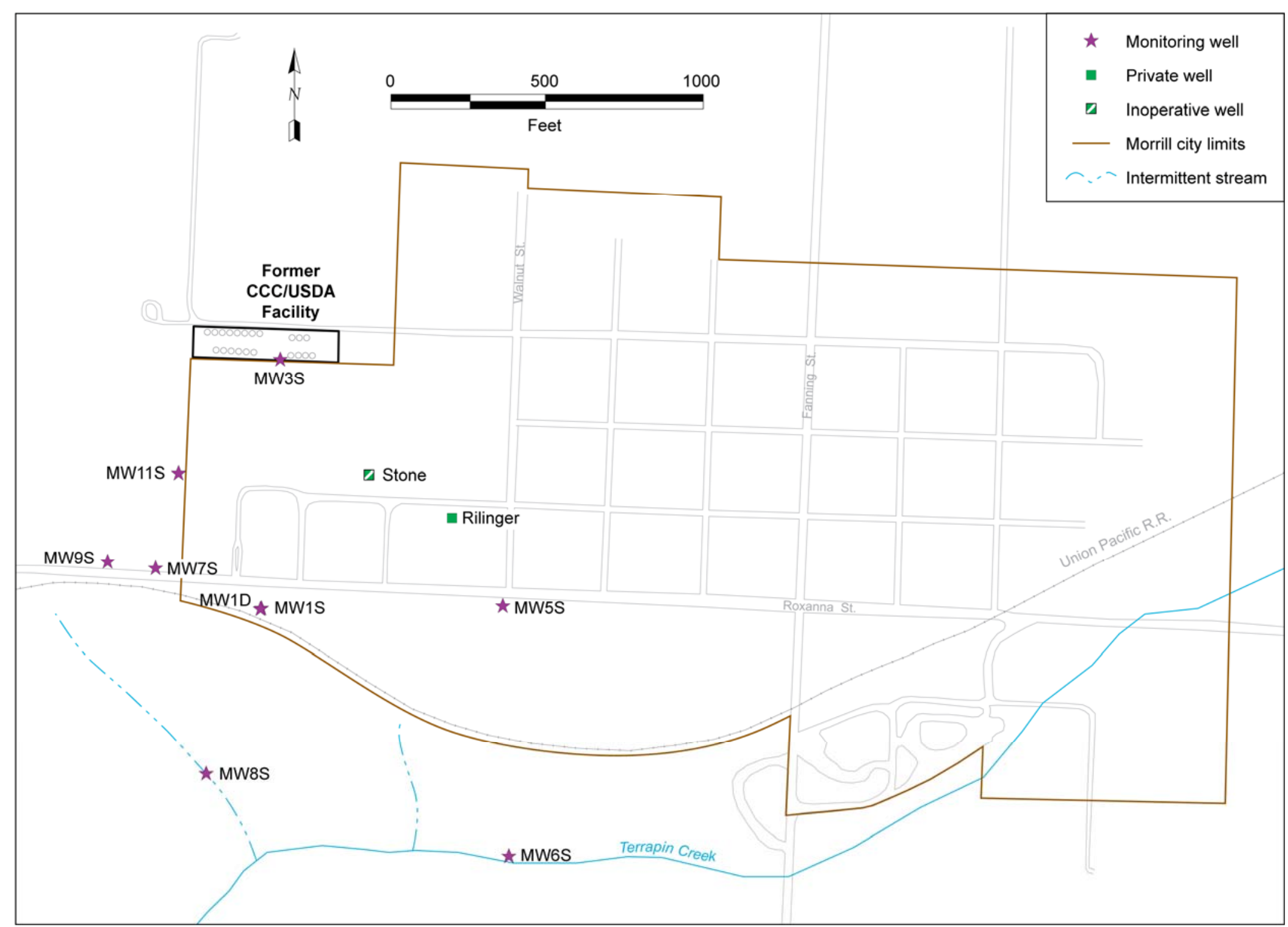




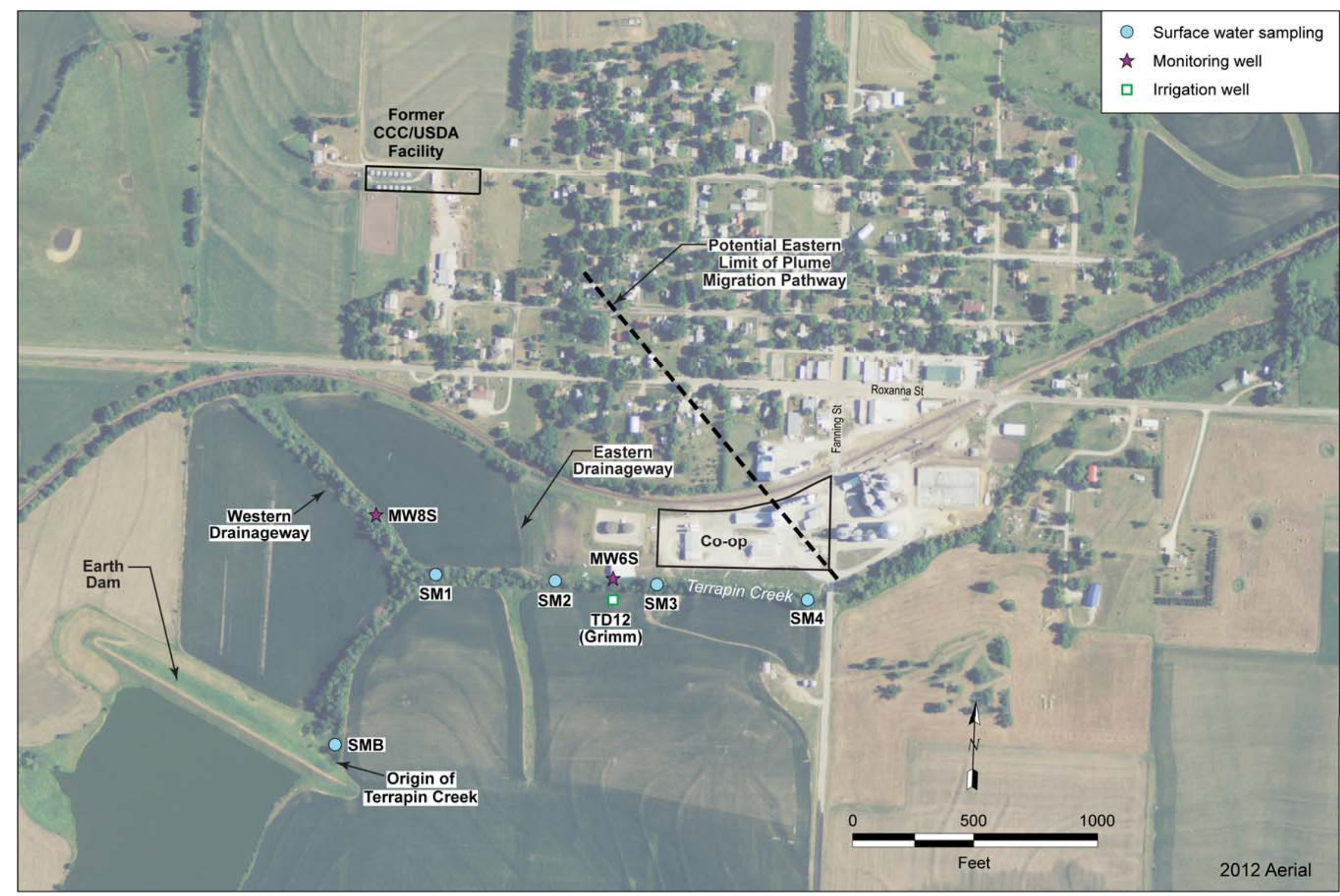

FIGURE 2.2 Locations of surface water and creek bed sediment sampling along Terrapin Creek in 2016. 


\section{Results and Discussion}

\subsection{Groundwater Level Data}

Depths to groundwater were measured manually in conjunction with the data recorder downloads on December 19, 2017, and during the annual groundwater sampling event on May 30-June 2, 2017. The manually measured data for 2015-2016 are in Table 3.1. Gaps appear in the automatically recorded water level data for several wells (MW1S, MW4S, and MW6S) because of battery or other electrical failures.

Potentiometric surface maps based on the water levels measured manually in May-June 2017 and December 2017 are in Figures 3.1 and 3.2, respectively. As in previous years, groundwater flow during spring 2017 (Figure 3.1, May 30-June 2, 2017) and winter 2017 (Figure 3.2, December 19, 2017) was predominantly to the south-southeast, from the vicinity of the former CCC/USDA facility toward Terrapin Creek.

Persistently low water levels observed at well MW11S (Argonne 2013, 2014, 2015, 2016, 2017) suggest the presence of a groundwater "sink" southwest of the former CCC/USDA facility, in the vicinity of this location. This observation is a reflection of the greater depth of completion of well MW11S in the aquifer unit and the local three-dimensional groundwater flow system at this location, relative to nearby wells MW3S, MW4S, and MW7S (Figure 3.3; see also Argonne 2014). To eliminate this influence on the interpreted lateral groundwater flow directions, the estimated potentiometric surface maps in Figures 3.1 and 3.2 were generated by excluding the water level measurements at MW11S.

The hydrographs in Figure 3.4 are based on the data generated in 2017. As in previous years, these hydrographs demonstrate that pumping at the Grimm irrigation well has a direct effect on the transient water levels at MW1D, MW06S, and MW11S. The recorder at MW11S was added late in 2013 to help document this effect.

In contrast with data from 2014, water levels showed a transient increase in the spring and early summer in 2017 (Figure 3.4), as in 2015-2016 and the years prior to 2014, which typically has occurred in response to periodic heavy rain events during this season. 


\subsection{Groundwater Analysis Results}

The analytical data for VOCs in the groundwater samples collected in May-June 2017 are in Table 3.2, together with data for the previous sampling events conducted under the KDHEapproved monitoring plan (Argonne 2005b). The results of field measurements for the groundwater samples are in Table 3.3. The May-June 2017 data for carbon tetrachloride in groundwater are illustrated in Figure 3.5, with the May 2016 data for comparison.

In May-June 2017 (Figure 3.5), carbon tetrachloride was detected at or above the maximum contaminant level (MCL) at three of the 11 locations sampled (MW1S, MW7S, and MW11S). Carbon tetrachloride concentrations in 2017 ranged from a minimum estimated concentration < $1.0 \mu \mathrm{g} / \mathrm{L}$ at the Rilinger private well to a maximum of $25 \mu \mathrm{g} / \mathrm{L}$ at well MW11S. The maximum carbon tetrachloride concentration at the former CCC/USDA facility (MW3S) occurred in 1995 at a level of $390 \mu \mathrm{g} / \mathrm{L}$. The concentration at MW3S remained notably lower in 2017 (2.2 $\mu \mathrm{g} / \mathrm{L})$, a decrease first observed in $2016(2.8 \mu \mathrm{g} / \mathrm{L})$, compared with 2015 (38 $\mu \mathrm{g} / \mathrm{L})$. The carbon tetrachloride concentration at MW1S was higher in 2017 (22 $\mu \mathrm{g} / \mathrm{L})$ compared with 2016 (9.4 $\mu \mathrm{g} / \mathrm{L})$. A low level of chloroform $(<1 \mu \mathrm{g} / \mathrm{L})$ was also detected in MW1S, but was not detected at any of the other 10 locations sampled (Table 3.2).

No quantifiable levels were detected in the wells monitoring drainage to Terrapin Creek (MW8S and MW6S). This has been the case since monitoring at these wells began in 2004 (Table $3.2)$.

\subsection{Surface Water and Sediment Analysis Results}

Table 3.4 presents the historical results of VOCs analyses of the surface water and shallow sediment samples collected along Terrapin Creek, which demonstrate that these surface waters remain uncontaminated by carbon tetrachloride. The Terrapin Creek surface waters and shallow sediments were not sampled in 2017, per an approved reduction in the frequency of sampling to every other year (KDHE 2016a). The next surface water and sediment sampling event will occur in 2018. 


\subsection{Private Well Evaluation Results}

Argonne conducted evaluations of the use and condition of the Allen, Manning, Manning/Cain, Rilinger, and Stone private wells on February 3, 2017. The current owners (or an individual familiar with the status of the wells) were contacted by telephone and surveyed regarding the use and condition of the wells. Table 3.5 summarizes the results of the private well evaluation. 
TABLE 3.1 Hand-measured groundwater levels in January 2015-December 2017.

\begin{tabular}{|c|c|c|c|c|c|c|c|c|c|}
\hline \multirow[b]{2}{*}{ Well } & \multirow[b]{2}{*}{$\begin{array}{l}\text { Top of Casing } \\
\text { Elevation } \\
\text { (ft AMSL) }\end{array}$} & \multicolumn{2}{|c|}{ January $14,2015^{a}$} & \multicolumn{2}{|c|}{$\begin{array}{c}\text { April 29-30 and May 6, } \\
2015\end{array}$} & \multicolumn{2}{|c|}{ September 21, 2015} & \multicolumn{2}{|c|}{ February 25, 2016} \\
\hline & & $\begin{array}{l}\text { Depth to } \\
\text { Water } \\
\text { (ft TOC) }\end{array}$ & $\begin{array}{l}\text { Groundwater } \\
\text { Elevation } \\
\text { (ft AMSL) }\end{array}$ & $\begin{array}{l}\text { Depth to } \\
\text { Water } \\
\text { (ft TOC) }\end{array}$ & $\begin{array}{l}\text { Groundwater } \\
\text { Elevation } \\
\text { (ft AMSL) }\end{array}$ & $\begin{array}{l}\text { Depth to } \\
\text { Water } \\
\text { (ft TOC) }\end{array}$ & $\begin{array}{l}\text { Groundwater } \\
\text { Elevation } \\
\text { (ft AMSL) }\end{array}$ & $\begin{array}{l}\text { Depth to } \\
\text { Water } \\
\text { (ft TOC) }\end{array}$ & $\begin{array}{c}\text { Groundwater } \\
\text { Elevation } \\
\text { (ft AMSL) }\end{array}$ \\
\hline MW1S & 1124.68 & 31.41 & 1093.27 & 29.60 & 1095.08 & 25.05 & 1099.63 & 22.35 & 1102.33 \\
\hline MW1D & 1124.63 & 33.92 & 1090.71 & 33.35 & 1091.28 & 59.47 & 1065.16 & 31.39 & 1093.24 \\
\hline MW2S & 1137.07 & 42.08 & 1094.99 & 38.80 & 1098.27 & 31.82 & 1105.25 & 31.16 & 1105.91 \\
\hline MW3S & 1135.76 & 37.78 & 1097.98 & 33.95 & 1101.81 & 24.37 & 1111.39 & 24.51 & 1111.25 \\
\hline MW4S & 1143.61 & 47.68 & 1095.93 & - & - & 34.06 & 1109.55 & 33.95 & 1109.66 \\
\hline MW5S & 1122.21 & 30.43 & 1091.78 & 26.50 & 1095.71 & 21.09 & 1101.12 & 19.72 & 1102.49 \\
\hline MW6S & 1090.97 & 6.24 & 1084.73 & 6.60 & 1084.37 & 17.48 & 1073.49 & 5.85 & 1085.12 \\
\hline MW7S & 1119.86 & 25.34 & 1094.52 & 23.40 & 1096.46 & 14.84 & 1105.02 & 14.62 & 1105.24 \\
\hline MW8S & 1098.53 & 5.78 & 1092.75 & 4.70 & 1093.83 & - & - & - & - \\
\hline MW9S & 1118.31 & 26.23 & 1092.08 & 25.70 & 1092.61 & 40.91 & 1077.40 & 21.60 & 1096.71 \\
\hline MW10S & 1110.78 & 16.91 & 1093.87 & 16.35 & 1094.43 & 28.37 & 1082.41 & 10.16 & 1100.62 \\
\hline \multirow[t]{2}{*}{ MW11S } & 1133.08 & 42.09 & 1090.99 & 40.90 & 1092.18 & 43.78 & 1089.30 & 34.44 & 1098.64 \\
\hline & & \multicolumn{2}{|c|}{ May 6, 2016} & \multicolumn{2}{|c|}{ December 15, 2016} & \multicolumn{2}{|c|}{ May 30-June 2, 2017} & \multicolumn{2}{|c|}{ December 19, 2017} \\
\hline Well & $\begin{array}{l}\text { Top of Casing } \\
\text { Elevation } \\
\text { (ft AMSL) }\end{array}$ & $\begin{array}{l}\text { Depth to } \\
\text { Water } \\
\text { (ft TOC) }\end{array}$ & $\begin{array}{l}\text { Groundwater } \\
\text { Elevation } \\
\text { (ft AMSL) }\end{array}$ & $\begin{array}{l}\text { Depth to } \\
\text { Water } \\
\text { (ft TOC) }\end{array}$ & $\begin{array}{l}\text { Groundwater } \\
\text { Elevation } \\
\text { (ft AMSL) }\end{array}$ & $\begin{array}{l}\text { Depth to } \\
\text { Water } \\
\text { (ft TOC) }\end{array}$ & $\begin{array}{c}\text { Groundwater } \\
\text { Elevation } \\
\text { (ft AMSL) }\end{array}$ & $\begin{array}{l}\text { Depth to } \\
\text { Water } \\
\text { (ft TOC) }\end{array}$ & $\begin{array}{l}\text { Groundwater } \\
\text { Elevation } \\
\text { (ft AMSL) }\end{array}$ \\
\hline MW1S & 1124.68 & 15.16 & 1109.52 & 23.56 & 1101.12 & 17.99 & 1106.69 & 29.82 & 1094.86 \\
\hline MW1D & 1124.63 & 29.72 & 1094.91 & 31.44 & 1093.19 & 29.19 & 1095.44 & 33.82 & 1090.81 \\
\hline MW2S & 1137.07 & 18.16 & 1118.91 & 32.73 & 1104.34 & 24.06 & 1113.01 & 40.82 & 1096.25 \\
\hline MW3S & 1135.76 & 12.60 & 1123.16 & 25.32 & 1110.44 & 17.79 & 1117.97 & 34.48 & 1101.28 \\
\hline MW4S & 1143.61 & 26.28 & 1117.33 & 34.13 & 1109.48 & 27.04 & 1116.57 & 42.03 & 1101.58 \\
\hline MW5S & 1122.21 & 8.27 & 1113.94 & 21.19 & 1101.02 & 12.02 & 1110.19 & 28.89 & 1093.32 \\
\hline MW6S & 1090.97 & 5.36 & 1085.61 & 5.76 & 1085.21 & 5.93 & 1085.04 & 6.02 & 1084.95 \\
\hline MW7S & 1119.86 & 10.03 & 1109.83 & 14.74 & 1105.12 & 10.02 & 1109.84 & 22.89 & 1096.97 \\
\hline MW8S & 1098.53 & 0.70 & 1097.83 & 2.55 & 1095.98 & 0.84 & 1097.69 & 5.03 & 1093.50 \\
\hline MW9S & 1118.31 & 19.00 & 1099.31 & 21.91 & 1096.40 & 18.65 & 1099.66 & 25.19 & 1093.12 \\
\hline MW10S & 1110.78 & 8.25 & 1102.53 & 10.89 & 1099.89 & 8.13 & 1102.65 & 15.68 & 1095.10 \\
\hline MW11S & 1133.08 & 29.75 & 1103.33 & 34.71 & 1098.37 & 30.06 & 1103.02 & 42.63 & 1090.45 \\
\hline
\end{tabular}

a Measurements planned for December 2014 were delayed until January 2015, because of Argonne commitments at other CCC/USDA sites. 
TABLE 3.2 Results of analyses at the AGEM Laboratory for VOCs in groundwater samples collected in 2003-2017.

\begin{tabular}{|c|c|c|c|c|c|c|c|c|c|c|c|c|}
\hline \multirow[b]{2}{*}{ Location } & \multirow{2}{*}{$\begin{array}{l}\text { Screen } \\
\text { Interval } \\
\text { (ft BGL) }\end{array}$} & \multirow[b]{2}{*}{$\begin{array}{l}\text { Sample } \\
\text { Date }\end{array}$} & \multicolumn{2}{|c|}{ Depth (ft TOC) } & \multirow[b]{2}{*}{$\begin{array}{l}\text { Volume } \\
\text { Purged }\end{array}$} & \multirow[b]{2}{*}{$\begin{array}{l}\text { Purge } \\
\text { Units }\end{array}$} & \multirow{2}{*}{$\begin{array}{l}\text { Pump } \\
\text { Intake } \\
\text { Position } \\
\text { (ft BGL) }\end{array}$} & \multicolumn{3}{|c|}{ Concentration $(\mu \mathrm{g} / \mathrm{L})$} & \multirow[b]{2}{*}{ Comment $^{\mathrm{a}}$} & \multirow[b]{2}{*}{ Sample } \\
\hline & & & To Water & Well & & & & $\begin{array}{c}\text { Carbon } \\
\text { Tetrachloride }\end{array}$ & Chloroform & $\begin{array}{l}\text { Methylene } \\
\text { Chloride }\end{array}$ & & \\
\hline MW1S & $11-51$ & $10 / 23 / 03$ & 30.36 & 54.04 & 70 & gal & - & 33 & 1.6 & $N D^{b}$ & TWV & MRMW1S-W-16422 \\
\hline MW1S & $11-51$ & $06 / 02 / 04$ & 26.97 & 53.94 & 53 & gal & - & 19 & 0.9 & ND & TWV & MRMW1S-W-16461 \\
\hline MW1S & $11-51$ & 09/13/05 & 24.16 & 53.9 & 57 & gal & - & 35 & 1.7 & ND & TWV & MRMW1S-W-19259 \\
\hline MW1S & $11-51$ & $03 / 22 / 06$ & 29.00 & 53.95 & 48 & gal & - & 40 & 1.8 & ND & TWV & MRMW1S-W-20008 \\
\hline MW1S & $11-51$ & 09/20/06 & 26.82 & 53.97 & 55 & gal & - & 23 & $0.9 \mathrm{Jc}^{\mathrm{c}}$ & ND & TWV & MRMW1S-W-22495 \\
\hline MW1S & $11-51$ & $03 / 21 / 07$ & 25.80 & 53.98 & 55 & gal & - & 23 & 1.1 & ND & TWV & MRMW1S-W-16488 \\
\hline MW1S & $11-51$ & $10 / 01 / 07$ & 21.65 & 53.95 & 63 & gal & - & 56 & 2.7 & ND & TWV & MRMW1S-W-16595 \\
\hline MW1S & $11-51$ & $04 / 14 / 08$ & 16.20 & 54 & 5.5 & $\mathrm{~L}$ & - & $0.3 \mathrm{~J}$ & ND & ND & LF & MRMW1S-W-23230 \\
\hline MW1S & $11-51$ & $04 / 22 / 08$ & 16.00 & 54 & 6.25 & $\mathrm{~L}$ & - & $0.2 \mathrm{~J}$ & ND & ND & LF & MRMW1S-W-23259 \\
\hline MW1S & $11-51$ & 05/01/08 & - & - & 3.24 & L & 22 & ND & ND & ND & LF-U & MRMW1S-22-W-23275 \\
\hline MW1S & $11-51$ & 05/01/08 & - & - & 3.24 & $\mathrm{~L}$ & 27 & ND & ND & ND & LF-M & MRMW1S-27-W-23276 \\
\hline MW1S & $11-51$ & 05/01/08 & - & - & 4.25 & $\mathrm{~L}$ & 48 & $0.3 \mathrm{~J}$ & ND & ND & LF-D & MRMW1S-48-W-23277 \\
\hline MW1S & $11-51$ & $10 / 20 / 08$ & 25.80 & 54 & 6 & L & 31 & $0.7 \mathrm{~J}$ & ND & ND & LF & MRMW1S-W-27620 \\
\hline MW1S & $11-51$ & $10 / 21 / 08$ & - & - & - & - & - & 35 & 1.8 & ND & TWV & MRMW1S-W-27649 \\
\hline MW1S & $11-51$ & $04 / 24 / 09$ & 24.40 & 54 & 5 & $\mathrm{~L}$ & 39.2 & ND & ND & ND & LF & MRMW1S-W-27652 \\
\hline MW1S & $11-51$ & 09/03/09 & 19.00 & 54 & 8 & $\mathrm{~L}$ & 35 & ND & ND & ND & LF & MRMW1S-W-29942 \\
\hline MW1S & $11-51$ & 09/04/09 & 19.30 & 51.2 & 244 & $\mathrm{~L}$ & 50 & 34 & 1.7 & ND & TWV & MRMW1S-W-29971 \\
\hline MW1S & $11-51$ & $04 / 07 / 10$ & 11.65 & 51.3 & 7 & $\mathrm{~L}$ & 16.6 & ND & ND & ND & LF-U & MRMW1ST-W-29981 \\
\hline MW1S & $11-51$ & $04 / 07 / 10$ & 11.57 & 51.31 & 6 & $\mathrm{~L}$ & 31.5 & ND & ND & ND & LF-M & MRMW1SM-W-29980 \\
\hline MW1S & $11-51$ & $04 / 07 / 10$ & 11.65 & 51.31 & 6 & $\mathrm{~L}$ & 46.3 & ND & ND & ND & LF-D & MRMW1SB-W-29979 \\
\hline MW1S & $11-51$ & $04 / 07 / 10$ & 11.48 & 51.3 & 80 & gal & 49 & 21 & 1.2 & ND & TWV & MRMW1S3X-W-29982 \\
\hline MW1S & $11-51$ & $09 / 22 / 10$ & 19.89 & 54 & 10 & $\mathrm{~L}$ & 31 & 1.6 & ND & ND & LF & MRMW1S-W-30010 \\
\hline MW1S & $11-51$ & $04 / 20 / 11$ & 23.45 & 54 & 6.5 & $\mathrm{~L}$ & 37 & $0.3 \mathrm{~J}$ & ND & ND & LF & MRMW1S-W-30038 \\
\hline MW1S & $11-51$ & $10 / 04 / 11$ & 21.80 & 54 & 8 & $\mathrm{~L}$ & 34.9 & 1.7 & ND & ND & LF & MRMW1S-W-30067 \\
\hline MW1S & $11-51$ & $10 / 12 / 11$ & 22.14 & 54 & 6 & L & 36.57 & 1.5 & ND & ND & LF & MRMW1S-W-30091 \\
\hline MW1S & $11-51$ & $10 / 12 / 11$ & 22.41 & 54 & 65 & gal & 49 & 23 & 1.2 & ND & TWV & MRMW1S3X-W-30093 \\
\hline MW1S & $11-51$ & $03 / 29 / 12$ & 20.10 & 54 & 8 & $\mathrm{~L}$ & 25 & ND & $\mathrm{ND}$ & ND & LF-U & MRMW1SU-W-30099 \\
\hline MW1S & $11-51$ & $03 / 29 / 12$ & 20.17 & 54 & 8 & $\mathrm{~L}$ & 35 & $0.3 \mathrm{~J}$ & ND & ND & LF-M & MRMW1SM-W-30100 \\
\hline MW1S & $11-51$ & $03 / 29 / 12$ & 20.17 & 54 & 5.5 & $\mathrm{~L}$ & 46 & ND & ND & ND & LF-D & MRMW1SL-W-30101 \\
\hline MW1S & $11-51$ & $03 / 29 / 12$ & 20.15 & 54 & 68 & gal & 50 & 22 & 1.2 & ND & TWV & MRMW1S3X-W-30102 \\
\hline MW1S & $11-51$ & $05 / 07 / 13$ & 20.94 & 54 & 6 & $\mathrm{~L}$ & 28.46 & ND & ND & ND & LF-U & MRMW1SU-W-30136 \\
\hline MW1S & $11-51$ & $05 / 07 / 13$ & 20.94 & 54 & 6 & $\mathrm{~L}$ & 35.97 & ND & ND & ND & LF-M & MRMW1SM-W-30137 \\
\hline MW1S & $11-51$ & $05 / 07 / 13$ & 20.94 & 54 & 6 & L & 43.49 & ND & ND & ND & LF-D & MRMW1SL-W-30138 \\
\hline MW1S & $11-51$ & $05 / 07 / 13$ & 20.94 & 54 & 65 & gal & - & 2.7 & ND & ND & TWV & MRMW1S3X-W-30139 \\
\hline MW1S & 20 & $05 / 06 / 13$ & - & - & - & - & - & ND & ND & ND & PDB & MRMW1SA-W-30169 \\
\hline MW1S & 22 & $05 / 06 / 13$ & - & - & - & - & - & ND & ND & ND & PDB & MRMW1SB-W-30170 \\
\hline MW1S & 24 & $05 / 06 / 13$ & - & - & - & - & - & ND & ND & ND & PDB & MRMW1SC-W-30171 \\
\hline MW1S & 26 & $05 / 06 / 13$ & - & - & - & - & - & ND & ND & ND & PDB & MRMW1SD-W-30172 \\
\hline MW1S & 28 & 05/06/13 & - & - & - & - & - & ND & $0.3 \mathrm{~J}$ & ND & PDB & MRMW1SE-W-30173 \\
\hline MW1S & 30 & $05 / 06 / 13$ & - & - & - & - & - & ND & ND & ND & PDB & MRMW1SF-W-30174 \\
\hline MW1S & 32 & $05 / 06 / 13$ & - & - & - & - & - & ND & ND & ND & PDB & MRMW1SG-W-30175 \\
\hline
\end{tabular}


TABLE 3.2 (Cont.)

\begin{tabular}{|c|c|c|c|c|c|c|c|c|c|c|c|c|}
\hline \multirow[b]{3}{*}{ Location } & \multirow{3}{*}{$\begin{array}{l}\text { Screen } \\
\text { Interval } \\
\text { (ft BGL) }\end{array}$} & \multirow{3}{*}{$\begin{array}{l}\text { Sample } \\
\text { Date }\end{array}$} & \multirow{2}{*}{\multicolumn{2}{|c|}{ Depth (ft TOC) }} & \multirow{3}{*}{$\begin{array}{l}\text { Volume } \\
\text { Purged }\end{array}$} & \multirow{3}{*}{$\begin{array}{l}\text { Purge } \\
\text { Units }\end{array}$} & \multirow{3}{*}{$\begin{array}{l}\text { Pump } \\
\text { Intake } \\
\text { Position } \\
\text { (ft BGL) }\end{array}$} & \multicolumn{3}{|c|}{ Concentration $(\mu \mathrm{g} / \mathrm{L})$} & \multirow[b]{3}{*}{ Comment $^{a}$} & \multirow[b]{3}{*}{ Sample } \\
\hline & & & & & & & & & & & & \\
\hline & & & To Water & Well & & & & Tetrachloride & Chloroform & Chloride & & \\
\hline MW1S & 34 & 05/06/13 & - & - & - & - & - & ND & ND & ND & PDB & MRMW1SH-W-30176 \\
\hline MW1S & 36 & 05/06/13 & - & - & - & - & - & ND & ND & ND & PDB & MRMW1SI-W-30177 \\
\hline MW1S & 38 & 05/06/13 & - & - & - & - & - & ND & ND & ND & PDB & MRMW1SJ-W-30178 \\
\hline MW1S & 40 & 05/06/13 & - & - & - & - & - & ND & ND & ND & PDB & MRMW1SK-W-30179 \\
\hline MW1S & 42 & 05/06/13 & - & - & - & - & - & ND & ND & ND & PDB & MRMW1SL-W-30180 \\
\hline MW1S & 44 & $05 / 06 / 13$ & - & - & - & - & - & ND & ND & ND & PDB & MRMW1SM-W-30181 \\
\hline MW1S & 46 & $05 / 06 / 13$ & - & - & - & - & - & ND & ND & ND & PDB & MRMW1SN-W-30182 \\
\hline MW1S & 48 & $05 / 06 / 13$ & - & - & - & - & - & ND & ND & ND & PDB & MRMW1SO-W-30183 \\
\hline MW1S & 50 & $05 / 06 / 13$ & - & - & - & - & - & ND & ND & ND & PDB & MRMW1SP-W-30184 \\
\hline MW1S & $11-51$ & $06 / 05 / 14$ & 27.38 & 54 & 7 & $\mathrm{~L}$ & 30.54 & ND & ND & ND & LF-U & MRMW1SU-W-31941 \\
\hline MW1S & $11-51$ & $06 / 05 / 14$ & 27.38 & 54 & 6 & $\mathrm{~L}$ & 37.38 & ND & ND & ND & LF-M & MRMW1SM-W-31942 \\
\hline MW1S & $11-51$ & $06 / 05 / 14$ & 27.38 & 54 & 6 & $\bar{L}$ & 44.18 & ND & ND & ND & LF-D & MRMW1SL-W-31943 \\
\hline MW1S & $11-51$ & $06 / 06 / 14$ & 27.38 & 54 & 65 & gal & 52 & 3.0 & $0.2 \mathrm{~J}$ & ND & TWV & MRMW1S3X-W-31944 \\
\hline MW1S & 20 & $06 / 04 / 14$ & - & - & - & - & - & ND & ND & ND & PDB & MRMW1SA-W-31925 \\
\hline MW1S & 22 & $06 / 04 / 14$ & - & - & - & - & - & ND & ND & ND & PDB & MRMW1SB-W-31926 \\
\hline MW1S & 24 & $06 / 04 / 14$ & - & - & - & - & - & ND & ND & ND & PDB & MRMW1SC-W-31927 \\
\hline MW1S & 26 & $06 / 04 / 14$ & - & - & - & - & - & ND & ND & ND & PDB & MRMW1SD-W-31928 \\
\hline MW1S & 28 & $06 / 04 / 14$ & - & - & - & - & - & ND & ND & ND & PDB & MRMW1SE-W-31929 \\
\hline MW1S & 30 & $06 / 04 / 14$ & - & - & - & - & - & ND & ND & ND & PDB & MRMW1SF-W-31930 \\
\hline MW1S & 32 & $06 / 04 / 14$ & - & - & - & - & - & ND & ND & ND & PDB & MRMW1SG-W-31931 \\
\hline MW1S & 34 & $06 / 04 / 14$ & - & - & - & - & - & ND & ND & ND & PDB & MRMW1SH-W-31932 \\
\hline MW1S & 36 & $06 / 04 / 14$ & - & - & - & - & - & ND & ND & ND & PDB & MRMW1SI-W-31933 \\
\hline MW1S & 38 & $06 / 04 / 14$ & - & - & - & - & - & ND & ND & ND & PDB & MRMW1SJ-W-31934 \\
\hline MW1S & 40 & $06 / 04 / 14$ & - & - & - & - & - & ND & ND & ND & PDB & MRMW1SK-W-31935 \\
\hline MW1S & 42 & $06 / 04 / 14$ & - & - & - & - & - & ND & ND & ND & PDB & MRMW1SL-W-31936 \\
\hline MW1S & 44 & $06 / 04 / 14$ & - & - & - & - & - & ND & ND & ND & PDB & MRMW1SM-W-31937 \\
\hline MW1S & 46 & $06 / 04 / 14$ & - & - & - & - & - & ND & ND & ND & PDB & MRMW1SN-W-31938 \\
\hline MW1S & 48 & $06 / 04 / 14$ & - & - & - & - & - & ND & ND & ND & PDB & MRMW1SO-W-31939 \\
\hline MW1S & 50 & $06 / 04 / 14$ & - & - & - & - & - & ND & ND & ND & PDB & MRMW1SP-W-31940 \\
\hline MW1S & $11-51$ & 04/30/15 & 29.6 & 54 & 6 & $\mathrm{~L}$ & 32.96 & ND & ND & ND & LF-U & MRMW1SU-W-37545 \\
\hline MW1S & $11-51$ & $04 / 30 / 15$ & 29.6 & 54 & 6 & $\mathrm{~L}$ & 38.98 & ND & ND & ND & LF-M & MRMW1SM-W-37546 \\
\hline MW1S & $11-51$ & 04/30/15 & 29.6 & 54 & 6 & $\mathrm{~L}$ & 44.99 & ND & ND & ND & LF-D & MRMW1SL-W-37547 \\
\hline MW1S & $11-51$ & $04 / 30 / 15$ & 29.6 & 54 & 60 & gal & 55 & 4.6 & ND & ND & TWV & MRMW1S3X-W-37548 \\
\hline MW1S & 20 & $04 / 28 / 15$ & - & - & - & - & - & ND & ND & ND & PDB & MRMW1S20-W-37529 \\
\hline MW1S & 22 & $04 / 28 / 15$ & - & - & - & - & - & ND & ND & ND & PDB & MRMW1S22-W-37530 \\
\hline MW1S & 24 & $04 / 28 / 15$ & - & - & - & - & - & ND & ND & ND & PDB & MRMW1S24-W-37531 \\
\hline MW1S & 26 & $04 / 28 / 15$ & - & - & - & - & - & ND & ND & ND & PDB & MRMW1S26-W-37532 \\
\hline MW1S & 28 & $04 / 28 / 15$ & - & - & - & - & - & ND & ND & ND & PDB & MRMW1S28-W-37533 \\
\hline MW1S & 30 & $04 / 28 / 15$ & - & - & - & - & - & ND & ND & ND & PDB & MRMW1S30-W-37534 \\
\hline MW1S & 32 & $04 / 28 / 15$ & - & - & - & - & - & ND & ND & ND & PDB & MRMW1S32-W-37535 \\
\hline MW1S & 34 & $04 / 28 / 15$ & - & - & - & - & - & ND & ND & ND & PDB & MRMW1S34-W-37536 \\
\hline
\end{tabular}


TABLE 3.2 (Cont.)

\begin{tabular}{|c|c|c|c|c|c|c|c|c|c|c|c|c|}
\hline \multirow[b]{2}{*}{ Location } & \multirow{2}{*}{$\begin{array}{l}\text { Screen } \\
\text { Interval } \\
\text { (ft BGL) }\end{array}$} & \multirow[b]{2}{*}{$\begin{array}{l}\text { Sample } \\
\text { Date }\end{array}$} & \multicolumn{2}{|c|}{ Depth (ft TOC) } & \multirow[b]{2}{*}{$\begin{array}{l}\text { Volume } \\
\text { Purged }\end{array}$} & \multirow[b]{2}{*}{$\begin{array}{l}\text { Purge } \\
\text { Units }\end{array}$} & \multirow{2}{*}{$\begin{array}{l}\text { Pump } \\
\text { Intake } \\
\text { Position } \\
\text { (ft BGL) }\end{array}$} & \multicolumn{3}{|c|}{ Concentration $(\mu \mathrm{g} / \mathrm{L})$} & \multirow[b]{2}{*}{ Comment $^{\mathrm{a}}$} & \multirow[b]{2}{*}{ Sample } \\
\hline & & & To Water & Well & & & & $\begin{array}{c}\text { Carbon } \\
\text { Tetrachloride }\end{array}$ & Chloroform & $\begin{array}{l}\text { Methylene } \\
\text { Chloride }\end{array}$ & & \\
\hline MW1S & 36 & $04 / 28 / 15$ & - & - & - & - & - & ND & ND & ND & PDB & MRMW1S28-W-37537 \\
\hline MW1S & 38 & $04 / 28 / 15$ & - & - & - & - & - & ND & ND & ND & PDB & MRMW1S30-W-37538 \\
\hline MW1S & 40 & $04 / 28 / 15$ & - & - & - & - & - & ND & ND & ND & PDB & MRMW1S32-W-37539 \\
\hline MW1S & 42 & $04 / 28 / 15$ & - & - & - & - & - & ND & ND & ND & PDB & MRMW1S34-W-37540 \\
\hline MW1S & 44 & $04 / 28 / 15$ & - & - & - & - & - & ND & ND & ND & PDB & MRMW1S28-W-37541 \\
\hline MW1S & 46 & $04 / 28 / 15$ & - & - & - & - & - & ND & ND & ND & PDB & MRMW1S30-W-37542 \\
\hline MW1S & 48 & $04 / 28 / 15$ & - & - & - & - & - & ND & ND & ND & PDB & MRMW1S32-W-37543 \\
\hline MW1S & 50 & $04 / 28 / 15$ & - & - & - & - & - & ND & ND & ND & PDB & MRMW1S34-W-37544 \\
\hline MW1S & $11-51$ & $05 / 08 / 16$ & 15.50 & 54 & 6.5 & $\mathrm{~L}$ & 34.11 & ND & ND & ND & LF-U & MRMW1SU-W-39163 \\
\hline MW1S & $11-51$ & 05/08/16 & 15.50 & 54 & 6.5 & $\mathrm{~L}$ & 40.13 & ND & ND & ND & LF-M & MRMW1SM-W-39164 \\
\hline MW1S & $11-51$ & $05 / 08 / 16$ & 15.50 & 54 & 6.5 & L & 46.14 & ND & ND & ND & LF-D & MRMW1SL-W-39165 \\
\hline MW1S & $11-51$ & $05 / 08 / 16$ & 15.50 & 54 & 80 & gal & 52 & 9.4 & $0.7 \mathrm{~J}$ & ND & TWV & MRMW1S3X-W-39166 \\
\hline MW1S & 20 & $05 / 08 / 16$ & - & - & - & - & - & ND & ND & ND & PDB & MRMW1S20-W-39147 \\
\hline MW1S & 22 & 05/08/16 & - & - & - & - & - & ND & ND & ND & PDB & MRMW1S22-W-39148 \\
\hline MW1S & 24 & $05 / 08 / 16$ & - & - & - & - & - & ND & ND & ND & PDB & MRMW1S24-W-39149 \\
\hline MW1S & 26 & $05 / 08 / 16$ & - & - & - & - & - & ND & ND & ND & PDB & MRMW1S26-W-39150 \\
\hline MW1S & 28 & $05 / 08 / 16$ & - & - & - & - & - & ND & ND & ND & PDB & MRMW1S28-W-39151 \\
\hline MW1S & 30 & $05 / 08 / 16$ & - & - & - & - & - & ND & ND & ND & PDB & MRMW1S30-W-39152 \\
\hline MW1S & 32 & $05 / 08 / 16$ & - & - & - & - & - & ND & ND & ND & PDB & MRMW1S32-W-39153 \\
\hline MW1S & 34 & $05 / 08 / 16$ & - & - & - & - & - & ND & ND & ND & PDB & MRMW1S34-W-39154 \\
\hline MW1S & 36 & 05/08/16 & - & - & - & - & - & ND & ND & ND & PDB & MRMW1S36-W-39155 \\
\hline MW1S & 38 & $05 / 08 / 16$ & - & - & - & - & - & ND & ND & ND & PDB & MRMW1S38-W-39156 \\
\hline MW1S & 40 & 05/08/16 & - & - & - & - & - & ND & ND & ND & PDB & MRMW1S40-W-39157 \\
\hline MW1S & 42 & $05 / 08 / 16$ & - & - & - & - & - & ND & ND & ND & PDB & MRMW1S42-W-39158 \\
\hline MW1S & 44 & 05/08/16 & - & - & - & - & - & ND & ND & ND & PDB & MRMW1S44-W-39159 \\
\hline MW1s & 46 & $05 / 08 / 16$ & - & - & - & - & - & ND & ND & ND & PDB & MRMW1S46-W-39160 \\
\hline MW1S & 48 & 05/08/16 & - & - & - & - & - & ND & ND & ND & PDB & MRMW1S48-W-39161 \\
\hline MW1S & 50 & $05 / 08 / 16$ & - & - & - & - & - & ND & ND & ND & PDB & MRMW1S50-W-39162 \\
\hline $\mathrm{MW} \mathrm{S}^{\mathrm{h}}$ & $11-51$ & $06 / 01 / 17$ & 17.55 & 54 & 72 & gal & 52 & 22 & $0.7 \mathrm{~J}$ & ND & TWV & MRMW1S50-W-39789 \\
\hline MW1D & $63-88$ & $10 / 22 / 03$ & 28.39 & 88.5 & 92 & gal & - & ND & ND & ND & TWV & MRMW1D-W-16421 \\
\hline MW1D & $63-88$ & $06 / 02 / 04$ & 26.82 & 88.6 & 140 & gal & - & ND & ND & ND & TWV & MRMW1D-W-16458 \\
\hline MW1D & $63-88$ & $09 / 13 / 05$ & 23.72 & 88.6 & 200 & gal & - & ND & ND & ND & TWV & MRMW1D-W-16518 \\
\hline MW1D & $63-88$ & 03/19/06 & 26.85 & 88.6 & 112 & gal & - & ND & ND & $0.4 \mathrm{~J} \mathrm{~B}^{\mathrm{d}}$ & TWV & MRMW1D-W-19986 \\
\hline MW1D & $63-88$ & 09/20/06 & 25.52 & 88.8 & 125 & gal & - & ND & ND & ND & TWV & MRMW1D-W-16532 \\
\hline MW1D & $63-88$ & 03/21/07 & 25.79 & 88.8 & 125 & gal & - & ND & ND & ND & TWV & MRMW1D-W-16487 \\
\hline MW1D & $63-88$ & $10 / 01 / 07$ & 22.75 & 89.38 & 130 & gal & - & ND & ND & ND & TWV & MRMW1D-W-16596 \\
\hline MW1D & $63-88$ & $04 / 14 / 08$ & 29.51 & 89 & 6 & $\mathrm{~L}$ & - & ND & ND & ND & LF & MRMW1D-W-23231 \\
\hline MW1D & $63-88$ & $10 / 20 / 08$ & 30.40 & 89 & 7 & $\mathrm{~L}$ & 75.5 & ND & ND & ND & LF & MRMW1D-W-27621 \\
\hline MW1D & $63-88$ & $04 / 24 / 09$ & 31.00 & 89 & 7 & L & 75.5 & ND & ND & ND & LF & MRMW1D-W-27653 \\
\hline MW1D & $63-88$ & 09/03/09 & 27.05 & 89 & 6.5 & $\mathrm{~L}$ & 75.5 & ND & ND & ND & LF & MRMW1D-W-29943 \\
\hline
\end{tabular}


TABLE 3.2 (Cont.)

\begin{tabular}{|c|c|c|c|c|c|c|c|c|c|c|c|c|}
\hline \multirow[b]{2}{*}{ Location } & \multirow{2}{*}{$\begin{array}{l}\text { Screen } \\
\text { Interval } \\
\text { (ft BGL) }\end{array}$} & \multirow[b]{2}{*}{$\begin{array}{l}\text { Sample } \\
\text { Date }\end{array}$} & \multicolumn{2}{|c|}{ Depth (ft TOC) } & \multirow[b]{2}{*}{$\begin{array}{l}\text { Volume } \\
\text { Purged }\end{array}$} & \multirow[b]{2}{*}{$\begin{array}{l}\text { Purge } \\
\text { Units }\end{array}$} & \multirow{2}{*}{$\begin{array}{l}\text { Pump } \\
\text { Intake } \\
\text { Position } \\
\text { (ft BGL) }\end{array}$} & \multicolumn{3}{|c|}{ Concentration $(\mu \mathrm{g} / \mathrm{L})$} & \multirow[b]{2}{*}{ Comment $^{\mathrm{a}}$} & \multirow[b]{2}{*}{ Sample } \\
\hline & & & To Water & Well & & & & $\begin{array}{c}\text { Carbon } \\
\text { Tetrachloride }\end{array}$ & Chloroform & $\begin{array}{l}\text { Methylene } \\
\text { Chloride }\end{array}$ & & \\
\hline MW1D & $63-88$ & 04/06/10 & 24.70 & 89 & 8.5 & $\mathrm{~L}$ & 75.5 & ND & ND & ND & LF & MRMW1D-W-29983 \\
\hline MW1D & $63-88$ & $09 / 22 / 10$ & 27.92 & 89 & 9 & $\mathrm{~L}$ & 75.5 & ND & ND & ND & LF & MRMW1D-W-30011 \\
\hline MW1D & $63-88$ & $04 / 20 / 11$ & 30.15 & 89 & 5.5 & $\mathrm{~L}$ & 75.5 & ND & ND & ND & LF & MRMW1D-W-30039 \\
\hline MW1D & $63-88$ & $10 / 04 / 11$ & 29.06 & 89 & 6.5 & L & 75.5 & ND & ND & ND & LF & MRMW1D-W-30068 \\
\hline MW1D & $63-88$ & $03 / 28 / 12$ & 30.50 & 89 & 5.8 & $\mathrm{~L}$ & 75.5 & ND & ND & ND & LF & MRMW1D-W-30103 \\
\hline MW1D & $63-88$ & $05 / 07 / 13$ & 28.98 & 89 & 6 & $\bar{L}$ & 75.5 & ND & ND & ND & LF & MRMW1D-W-30140 \\
\hline MW1D & $63-88$ & $06 / 06 / 14$ & 33.23 & 89 & 7 & $\mathrm{~L}$ & 75.5 & ND & ND & ND & LF & MRMW1D-W-31945 \\
\hline MW1D & $63-88$ & $05 / 06 / 15$ & 33.35 & 89 & 6 & $\mathrm{~L}$ & 75.5 & ND & ND & ND & LF & MRMW1D-W-37549 \\
\hline MW1D & $63-88$ & 05/07/16 & 29.72 & 89 & 5.25 & $\mathrm{~L}$ & 75.5 & ND & ND & ND & LF & MRMW1D-W-39167 \\
\hline MW1D & $63-88$ & $06 / 02 / 17$ & 29.19 & 89 & 6 & $\mathrm{~L}$ & 75.5 & ND & ND & ND & LF & MRMW1D-W-39790 \\
\hline MW2S & $13-53$ & $10 / 22 / 03$ & 42.21 & 53.35 & & & & ND & ND & ND & PDS & MRMW02-W-16419 \\
\hline MW2S & $13-53$ & $06 / 02 / 04$ & 37.44 & 53.3 & 31 & gal & - & ND & ND & ND & TWV & MRMW2S-W-16459 \\
\hline MW2S & $13-53$ & $09 / 14 / 05$ & 33.68 & 53.33 & 38 & gal & - & ND & ND & ND & TWV & MRMW2S-W-19264 \\
\hline MW2S & $13-53$ & $03 / 21 / 06$ & 40.87 & 53.32 & 27 & gal & - & ND & ND & ND & TWV & MRMW2S-W-19992 \\
\hline MW2S & $13-53$ & 09/18/06 & 36.53 & 53.3 & 28 & gal & - & ND & ND & ND & TWV & MRMW2S-W-22488 \\
\hline MW2S & $13-53$ & $03 / 22 / 07$ & 35.77 & 53.3 & 35 & gal & - & ND & ND & ND & TWV & MRMW2S-W-16559 \\
\hline MW2S & $13-53$ & $10 / 03 / 07$ & 31.17 & 53.4 & 44 & gal & - & ND & ND & ND & TWV & MRMW2S-W-16587 \\
\hline MW2S & $13-53$ & $04 / 15 / 08$ & 23.55 & 53.4 & 2.22 & $\mathrm{~L}$ & - & ND & ND & ND & LF & MRMW2S-W-23232 \\
\hline MW2S & $13-53$ & $10 / 21 / 08$ & 33.70 & 53.5 & 5 & $\mathrm{~L}$ & 33 & ND & ND & ND & LF & MRMW2S-W-27622 \\
\hline MW2S & $13-53$ & $10 / 21 / 08$ & - & - & - & - & - & ND & ND & ND & TWV & MRMW2S-W-27652 \\
\hline MW2S & $13-53$ & $04 / 23 / 09$ & 33.20 & 53.5 & 6.5 & L & 43.35 & ND & $0.6 \mathrm{~J}$ & ND & LF & MRMW2S-W-27654 \\
\hline MW2S & $13-53$ & 09/03/09 & 29.40 & 53.5 & 5.4 & $\mathrm{~L}$ & 41.2 & ND & ND & ND & LF & MRMW2S-W-29944 \\
\hline MW2S & $13-53$ & $04 / 06 / 10$ & 18.57 & 52.42 & 6.5 & $\mathrm{~L}$ & 36 & ND & ND & ND & LF & MRMW2S-W-29984 \\
\hline MW2S & $13-53$ & $09 / 22 / 10$ & 31.25 & 53 & 7 & $\mathrm{~L}$ & 33 & ND & ND & ND & LF & MRMW2S-W-30012 \\
\hline MW2S & $13-53$ & $04 / 20 / 11$ & 36.00 & 53.5 & 8 & $\mathrm{~L}$ & 44.5 & ND & ND & ND & LF & MRMW2S-W-30040 \\
\hline MW2S & $13-53$ & $10 / 04 / 11$ & 32.73 & 53.5 & 7.5 & $\mathrm{~L}$ & 42.6 & ND & ND & ND & LF & MRMW2S-W-30069 \\
\hline MW2S & $13-53$ & $03 / 28 / 12$ & 31.45 & 53.5 & 7 & L & 42.48 & ND & ND & ND & LF & MRMW2S-W-30104 \\
\hline MW2S & $13-53$ & $05 / 07 / 13$ & 32.86 & 53.5 & 5 & $\mathrm{~L}$ & 42.93 & ND & ND & ND & LF & MRMW2S-W-30141 \\
\hline MW2S & $13-53$ & $06 / 06 / 14$ & 37.03 & 53.5 & 8 & $\mathrm{~L}$ & 45.42 & ND & ND & ND & LF & MRMW2S-W-31946 \\
\hline MW2S & $13-53$ & 05/06/15 & 38.80 & 53.5 & 7 & L & 46.05 & ND & ND & ND & LF & MRMW2S-W-31950 \\
\hline MW2Si & $13-53$ & $05 / 07 / 16$ & 18.16 & 53.5 & 7 & $\overline{\mathrm{L}}$ & 46.05 & ND & ND & ND & LF & MRMW2S-W-39168 \\
\hline MW3S & $18-48$ & 10/23/03 & 36.47 & 47.79 & 73 & gal & - & 89 & 2.7 & ND & TWV & MRMW03-W-16423 \\
\hline MW3S & $18-48$ & $06 / 02 / 04$ & 30.67 & 47.5 & 34 & gal & - & 110 & 3.2 & ND & TWV & MRMW3S-W-16462 \\
\hline MW3S & $18-48$ & 09/13/05 & 25.60 & 47.6 & 50 & gal & - & 101 & 3.2 & ND & TWV & MRMW3S-W-19261 \\
\hline MW3S & $18-48$ & $03 / 23 / 06$ & 35.62 & 47.74 & 28 & gal & - & 91 & 2.6 & ND & TWV & MRMW3S-W-19994 \\
\hline MW3S & $18-48$ & 09/20/06 & 29.44 & 47.75 & 22 & gal & - & 49 & 1.5 & ND & TWV & MRMW3S-W-22496 \\
\hline MW3S & $18-48$ & $03 / 22 / 07$ & 26.19 & 47.75 & 45 & gal & - & 84 & 2.3 & ND & TWV & MRMW3S-W-16563 \\
\hline MW3S & $18-48$ & $10 / 03 / 07$ & 22.70 & 47.9 & 50 & gal & - & 61 & 2.0 & ND & TWV & MRMW3S-W-16585 \\
\hline MW3S & $18-48$ & $04 / 14 / 08$ & 16.95 & 47.8 & 3.31 & $\mathrm{~L}$ & - & 8.2 & $0.4 \mathrm{~J}$ & ND & LF & MRMW3S-W-23233 \\
\hline
\end{tabular}


TABLE 3.2 (Cont.)

\begin{tabular}{|c|c|c|c|c|c|c|c|c|c|c|c|c|}
\hline \multirow[b]{3}{*}{ Location } & \multirow{3}{*}{$\begin{array}{l}\text { Screen } \\
\text { Interval } \\
\text { (ft BGL) }\end{array}$} & \multirow{3}{*}{$\begin{array}{c}\text { Sample } \\
\text { Date }\end{array}$} & \multirow{2}{*}{\multicolumn{2}{|c|}{ Depth (ft TOC) }} & \multirow{3}{*}{$\begin{array}{l}\text { Volume } \\
\text { Purged }\end{array}$} & \multirow{3}{*}{$\begin{array}{l}\text { Purge } \\
\text { Units }\end{array}$} & \multirow{3}{*}{$\begin{array}{l}\text { Pump } \\
\text { Intake } \\
\text { Position } \\
\text { (ft BGL) }\end{array}$} & \multicolumn{3}{|c|}{ Concentration $(\mu \mathrm{g} / \mathrm{L})$} & \multirow[b]{3}{*}{ Comment $^{a}$} & \multirow[b]{3}{*}{ Sample } \\
\hline & & & & & & & & & & & & \\
\hline & & & To Water & Well & & & & Tetrachloride & Chloroform & Chloride & & \\
\hline MW3S & $18-48$ & $04 / 22 / 08$ & 15.80 & 47.8 & 6.5 & $\mathrm{~L}$ & _- & $0.7 \mathrm{~J}$ & ND & ND & LF & MRMW3S-W-23260 \\
\hline MW3S & $18-48$ & 05/01/08 & - & - & 2.62 & $\mathrm{~L}$ & 26 & $0.4 \mathrm{~J}$ & ND & ND & LF-U & MRMW3S-26-W-23269 \\
\hline MW3S & $18-48$ & 05/01/08 & - & - & 2.75 & $\mathrm{~L}$ & 38 & $0.4 \mathrm{~J}$ & ND & ND & LF-M & MRMW3S-38-W-23270 \\
\hline MW3S & $18-48$ & 05/01/08 & - & - & 3.17 & $\mathrm{~L}$ & 45 & $0.5 \mathrm{~J}$ & ND & ND & LF-D & MRMW3S-45-W-23271 \\
\hline MW3S & $18-48$ & $10 / 21 / 08$ & 27.00 & 47.8 & 4.2 & $\mathrm{~L}$ & 33 & 55 & 1.4 & ND & LF & MRMW3S-W-27623 \\
\hline MW3S & $18-48$ & 10/21/08 & - & - & - & - & - & 63 & 1.6 & ND & TWV & MRMW3S-W-27650 \\
\hline MW3S & $18-48$ & $04 / 23 / 09$ & 26.70 & 47.8 & 5 & $\mathrm{~L}$ & 37.3 & 29 & 1.4 & ND & LF & MRMW3S-W-27655 \\
\hline MW3S & $18-48$ & 09/03/09 & 22.40 & 47.8 & 5.5 & $\mathrm{~L}$ & 35.2 & 30 & 1.1 & ND & LF & MRMW3S-W-29945 \\
\hline MW3S & $18-48$ & 09/04/09 & 22.55 & 47.8 & 190 & L & 46 & 28 & $0.9 \mathrm{~J}$ & ND & TWV & MRMW3S-W-29972 \\
\hline MW3S & $18-48$ & $04 / 06 / 10$ & 12.14 & 47.8 & 6 & L & 33 & 3.5 & $0.2 \mathrm{~J}$ & ND & LF & MRMW3S-W-29985 \\
\hline MW3S & $18-48$ & 09/23/10 & 23.73 & 47.8 & 7 & $\mathrm{~L}$ & 33 & 47 & 1.6 & ND & LF & MRMW3S-W-30013 \\
\hline MW3S & $18-48$ & $04 / 20 / 11$ & 32.06 & 47.8 & 6.2 & L & 40 & 33 & 1.2 & ND & $\mathrm{LF}$ & MRMW3S-W-30041 \\
\hline MW3S & $18-48$ & $10 / 04 / 11$ & 25.95 & 47.8 & 7 & $\mathrm{~L}$ & 37 & 49 & 1.8 & ND & LF & MRMW3S-W-30070 \\
\hline MW3S & $18-48$ & $03 / 28 / 12$ & 23.95 & 47.8 & 6.5 & $\mathrm{~L}$ & 35.88 & 58 & 2.0 & ND & $\mathrm{LF}$ & MRMW3S-W-30105 \\
\hline MW3S & $18-48$ & $05 / 07 / 13$ & 25.65 & 47.8 & 7 & $\mathrm{~L}$ & 36.83 & 57 & 1.9 & ND & LF & MRMW3S-W-30142 \\
\hline MW3S & $18-48$ & $06 / 06 / 14$ & 34.46 & 47.8 & 7.25 & $\mathrm{~L}$ & 41.39 & 46 & 1.5 & ND & LF & MRMW3S-W-31947 \\
\hline MW3S & $18-48$ & $04 / 30 / 15$ & 33.95 & 47.8 & 7 & $\mathrm{~L}$ & 42.1 & 38 & 1.5 & ND & LF & MRMW3S-W-37551 \\
\hline MW3S & $18-48$ & $05 / 07 / 16$ & 12.60 & 12.60 & 7 & $\mathrm{~L}$ & 42.1 & 2.8 & ND & ND & LF & MRMW3S-W-39169 \\
\hline MW3S & $18-48$ & $06 / 01 / 17$ & 17.79 & 47.8 & 6 & $\mathrm{~L}$ & 33.04 & 2.2 & ND & ND & LF & MRMW3S-W-39792 \\
\hline MW4S & $17-47$ & $10 / 21 / 03$ & 46.40 & 47.8 & - & - & - & ND & ND & ND & PDS & MRMW04-W-16418 \\
\hline MW4S & $17-47$ & $06 / 04 / 04$ & 43.21 & 47.8 & 10 & gal & - & ND & ND & ND & TWV & MRMW4S-W-16470 \\
\hline MW4S & $17-47$ & $09 / 14 / 05$ & 36.21 & 47.81 & 8 & gal & - & ND & ND & ND & TWV & MRMW4S-W-19262 \\
\hline MW4S & $17-47$ & 03/21/06 & 44.55 & 47.72 & 6 & gal & - & ND & ND & ND & TWV & MRMW4S-W-19993 \\
\hline MW4S & $17-47$ & 09/18/06 & 41.56 & 47.75 & 5.25 & gal & - & ND & ND & ND & TWV & MRMW4S-W-22487 \\
\hline MW4S & $17-47$ & $03 / 22 / 07$ & 38.74 & 47.75 & 6 & gal & - & ND & ND & ND & TWV & MRMW4S-W-16562 \\
\hline MW4S & $17-47$ & $10 / 03 / 07$ & 31.11 & 47.73 & 30 & gal & - & $0.5 \mathrm{~J} \mathrm{R}^{\mathrm{e}}$ & ND & ND & TWV & MRMW4S-W-16586 \\
\hline MW4S & $17-47$ & $01 / 11 / 08$ & - & - & - & - & _- & ND & ND & ND & TWV & MOMW4S-W-011108 \\
\hline MW4S & $17-47$ & $04 / 14 / 08$ & 26.32 & 47.85 & 2.48 & $\mathrm{~L}$ & - & ND & ND & ND & LF & MRMW4S-W-23234 \\
\hline MW4S & $17-47$ & $10 / 20 / 08$ & 36.70 & 47.8 & 8 & gal & - & ND & ND & ND & TWV & MRMW4S-W-27624 \\
\hline MW4S & $17-47$ & $04 / 23 / 09$ & 41.50 & 47.8 & 5 & $\mathrm{~L}$ & 44.65 & ND & ND & ND & LF & MRMW4S-W-27656 \\
\hline MW4S & $17-47$ & 09/04/09 & 31.60 & 47.8 & 6 & $\mathrm{~L}$ & 39.3 & ND & ND & ND & LF & MRMW4S-W-29946 \\
\hline MW4S & $17-47$ & $04 / 06 / 10$ & 21.80 & 47.85 & 5.5 & $\mathrm{~L}$ & 34.83 & ND & ND & ND & LF & MRMW4S-W-29986 \\
\hline MW4S & $17-47$ & $09 / 22 / 10$ & 33.15 & 47.8 & 6 & $\mathrm{~L}$ & 32 & ND & ND & ND & LF & MRMW4S-W-30014 \\
\hline MW4S & $17-47$ & $04 / 21 / 11$ & 42.39 & 47.8 & 5 & $\mathrm{~L}$ & 44.7 & ND & ND & ND & LF & MRMW4S-W-30042 \\
\hline MW4S & $17-47$ & $10 / 04 / 11$ & 35.60 & 47.8 & 6 & $\mathrm{~L}$ & 40.9 & ND & ND & ND & LF & MRMW4S-W-30071 \\
\hline MW4S & $17-47$ & $03 / 28 / 12$ & 35.10 & 47.8 & 7.2 & $\mathrm{~L}$ & 41.45 & ND & ND & ND & LF & MRMW4S-W-30106 \\
\hline MW4S & $17-47$ & 05/07/13 & 44.2 & 47.8 & 9 & L & 45.6 & ND & ND & ND & LF & MRMW4S-W-30143 \\
\hline MW4S & - & - & - & - & - & - & - & - & - & - & NS & - \\
\hline MW4S & $17-47$ & 04/30/15 & - & - & - & - & - & - & - & - & NS & - \\
\hline MW4S ${ }^{i}$ & $17-47$ & 05/08/16 & 26.28 & 47.8 & - & L & 40.88 & ND & ND & ND & LF & MRMW4S-W-30170 \\
\hline
\end{tabular}


TABLE 3.2 (Cont.)

\begin{tabular}{|c|c|c|c|c|c|c|c|c|c|c|c|c|}
\hline \multirow[b]{2}{*}{ Location } & \multirow{2}{*}{$\begin{array}{l}\text { Screen } \\
\text { Interval } \\
\text { (ft BGL) }\end{array}$} & \multirow[b]{2}{*}{$\begin{array}{l}\text { Sample } \\
\text { Date }\end{array}$} & \multicolumn{2}{|c|}{ Depth (ft TOC) } & \multirow[b]{2}{*}{$\begin{array}{l}\text { Volume } \\
\text { Purged }\end{array}$} & \multirow[b]{2}{*}{$\begin{array}{l}\text { Purge } \\
\text { Units }\end{array}$} & \multirow{2}{*}{$\begin{array}{l}\text { Pump } \\
\text { Intake } \\
\text { Position } \\
\text { (ft BGL) }\end{array}$} & \multicolumn{3}{|c|}{ Concentration $(\mu \mathrm{g} / \mathrm{L})$} & \multirow[b]{2}{*}{ Comment $^{\mathrm{a}}$} & \multirow[b]{2}{*}{ Sample } \\
\hline & & & To Water & Well & & & & $\begin{array}{c}\text { Carbon } \\
\text { Tetrachloride }\end{array}$ & Chloroform & $\begin{array}{l}\text { Methylene } \\
\text { Chloride }\end{array}$ & & \\
\hline MW5S & $15-55$ & $10 / 22 / 03$ & 31.40 & 55.72 & 48 & gal & - & 5.8 & ND & ND & TWV & MRMW05-W-16420 \\
\hline MW5S & $15-55$ & $06 / 02 / 04$ & 26.33 & 55.65 & $>57$ & gal & - & 7.0 & ND & ND & TWV & MRMW5S-W-16460 \\
\hline MW5S & $15-55$ & 09/13/05 & 22.66 & 54.2 & 75 & gal & - & 6.3 & $0.2 \mathrm{~J}$ & ND & TWV & MRMW5S-W-19260 \\
\hline MW5S & $15-55$ & $03 / 22 / 06$ & 28.64 & 54.51 & 50 & gal & - & 7.3 & $0.2 \mathrm{~J}$ & ND & TWV & MRMW5S-W-19996 \\
\hline MW5S & $15-55$ & $09 / 20 / 06$ & 25.43 & 54.63 & 52 & gal & - & 6.4 & $0.3 \mathrm{~J}$ & ND & TWV & MRMW5S-W-22493 \\
\hline MW5S & $15-55$ & $03 / 22 / 07$ & 25.14 & 54.56 & 58 & gal & - & 6.5 & $0.4 \mathrm{~J}$ & ND & TWV & MRMW5S-W-16569 \\
\hline MW5S & $15-55$ & $10 / 03 / 07$ & 19.55 & 54.65 & 68 & gal & - & 4.0 & $0.3 \mathrm{~J}$ & ND & TWV & MRMW5S-W-16588 \\
\hline MW5S & $15-55$ & $04 / 14 / 08$ & 11.20 & 54.6 & 6 & $\mathrm{~L}$ & _- & ND & ND & ND & LF & MRMW5S-W-23235 \\
\hline MW5S & $15-55$ & $04 / 23 / 08$ & 11.30 & 54.6 & 6.5 & L & - & ND & ND & ND & LF & MRMW5S-W-23266 \\
\hline MW5S & $15-55$ & 05/01/08 & - & - & 3.7 & L & 20 & ND & ND & ND & LF-U & MRMW5S-20-W-23272 \\
\hline MW5S & $15-55$ & 05/01/08 & - & - & 3.4 & L & 28 & ND & ND & ND & LF-M & MRMW5S-28-W-23273 \\
\hline MW5S & $15-55$ & 05/01/08 & - & - & 4 & L & 52 & ND & ND & ND & LF-D & MRMW5S-52-W-23274 \\
\hline MW5S & $15-55$ & $10 / 21 / 08$ & 22.50 & 54.6 & 7 & L & 35 & 1.7 & ND & ND & LF & MRMW5S-W-27625 \\
\hline MW5S & $15-55$ & $04 / 24 / 09$ & 22.10 & 54.6 & 5.5 & $\mathrm{~L}$ & 38.35 & ND & ND & ND & LF & MRMW5S-W-27657 \\
\hline MW5S & $15-55$ & 09/03/09 & 17.60 & 54.6 & 5.5 & L & 36.3 & ND & ND & ND & LF & MRMW5S-W-29947 \\
\hline MW5S & $15-55$ & $04 / 07 / 10$ & 8.32 & 54.5 & 5.5 & $\mathrm{~L}$ & 35 & ND & ND & ND & LF & MRMW5S-W-29987 \\
\hline MW5S & $15-55$ & $09 / 22 / 10$ & 19.25 & 55 & 6.5 & L & 35 & ND & 9.4 & ND & LF & MRMW5S-W-30015 \\
\hline MW5S & $15-55$ & $04 / 20 / 11$ & 24.43 & 54.6 & 7 & L & 36 & 1.3 & ND & ND & LF & MRMW5S-W-30043 \\
\hline MW5S & $15-55$ & $10 / 04 / 11$ & 21.10 & 54.6 & 6.5 & L & 38.25 & ND & ND & ND & $\mathrm{LF}$ & MRMW5S-W-30072 \\
\hline MW5S & $15-55$ & $03 / 28 / 12$ & 20.60 & 54.6 & 7 & $\mathrm{~L}$ & 37.6 & $0.6 \mathrm{~J}$ & ND & ND & LF & MRMW5S-W-30107 \\
\hline MW5S & $15-55$ & $05 / 07 / 13$ & 21.9 & 54.6 & 5 & L & 38.45 & ND & ND & ND & $\mathrm{LF}$ & MRMW5S-W-30144 \\
\hline MW5S & $15-55$ & 06/06/14 & 25.04 & 54.6 & 8 & L & 40.19 & 1.2 & ND & ND & LF & MRMW5S-W-31949 \\
\hline MW5S & $15-55$ & $05 / 06 / 15$ & 26.5 & 54.6 & 5 & L & 40.88 & ND & ND & ND & $\mathrm{LF}$ & MRMW5S-W-37553 \\
\hline MW5S & $15-55$ & $05 / 07 / 16$ & 8.27 & 54.6 & 6 & $\mathrm{~L}$ & 35 & ND & ND & ND & LF & MRMW5S-W-39171 \\
\hline MW5S & $15-55$ & $06 / 01 / 17$ & 12.02 & 54.6 & 6 & L & 35 & ND & ND & ND & LF & MRMW5S-W-39794 \\
\hline MW6S & $10-25$ & $06 / 03 / 04$ & 3.34 & 26.9 & 45 & gal & - & ND & ND & ND & TWV & MRMW6S-W-16465 \\
\hline MW6S & $10-25$ & $09 / 14 / 05$ & 4.70 & 26.9 & 43 & gal & - & ND & ND & ND & TWV & MRMW6S-W-19263 \\
\hline MW6S & $10-25$ & $03 / 20 / 06$ & 5.35 & 26.91 & 43 & gal & - & ND & ND & ND & TWV & MRMW6S-W-19990 \\
\hline MW6S & $10-25$ & 09/18/06 & 5.48 & 26.92 & 27 & gal & - & ND & ND & ND & TWV & MRMW6S-W-22486 \\
\hline MW6S & $10-25$ & $03 / 21 / 07$ & 5.42 & 26.92 & 30 & gal & - & ND & ND & ND & TWV & MRMW6S-W-16486 \\
\hline MW6S & $10-25$ & $10 / 02 / 07$ & 5.00 & 26.9 & 31 & gal & - & ND & ND & ND & TWV & MRMW6S-W-16583 \\
\hline MW6S & $10-25$ & $04 / 15 / 08$ & 5.15 & 26.9 & 2.5 & $\mathrm{~L}$ & - & ND & ND & ND & LF & MRMW6S-W-23236 \\
\hline MW6S & $10-25$ & $10 / 20 / 08$ & 5.70 & 26.9 & 5 & L & 17.5 & ND & ND & ND & LF & MRMW6S-W-27626 \\
\hline MW6S & $10-25$ & $04 / 24 / 09$ & 6.20 & 26.9 & 12 & L & 17.5 & ND & ND & ND & LF & MRMW6S-W-27658 \\
\hline MW6S & $10-25$ & $09 / 04 / 09$ & 5.85 & 26.9 & 5.4 & L & 17.5 & ND & ND & ND & $\mathrm{LF}$ & MRMW6S-W-29948 \\
\hline MW6S & $10-25$ & $04 / 06 / 10$ & 6.15 & 26.9 & 8 & $\mathrm{~L}$ & 17.5 & ND & ND & ND & LF & MRMW6S-W-29988 \\
\hline MW6S & $10-25$ & $09 / 22 / 10$ & 5.53 & 26.9 & 8.75 & $\mathrm{~L}$ & 17.5 & ND & ND & ND & LF & MRMW6S-W-30016 \\
\hline MW6S & $10-25$ & $04 / 20 / 11$ & 6.52 & 24.88 & 8 & L & 17.5 & ND & ND & ND & LF & MRMW6S-W-30044 \\
\hline MW6S & $10-25$ & $10 / 04 / 11$ & 6.49 & 26.9 & 6 & $\mathrm{~L}$ & 17.5 & ND & ND & ND & LF & MRMW6S-W-30073 \\
\hline
\end{tabular}


TABLE 3.2 (Cont.)

\begin{tabular}{|c|c|c|c|c|c|c|c|c|c|c|c|c|}
\hline \multirow[b]{3}{*}{ Location } & \multirow{3}{*}{$\begin{array}{l}\text { Screen } \\
\text { Interval } \\
\text { (ft BGL) }\end{array}$} & \multirow{3}{*}{$\begin{array}{c}\text { Sample } \\
\text { Date }\end{array}$} & \multirow{2}{*}{\multicolumn{2}{|c|}{ Depth (ft TOC) }} & \multirow{3}{*}{$\begin{array}{l}\text { Volume } \\
\text { Purged }\end{array}$} & \multirow{3}{*}{$\begin{array}{l}\text { Purge } \\
\text { Units }\end{array}$} & \multirow{3}{*}{$\begin{array}{l}\text { Pump } \\
\text { Intake } \\
\text { Position } \\
\text { (ft BGL) }\end{array}$} & \multicolumn{3}{|c|}{ Concentration $(\mu \mathrm{g} / \mathrm{L})$} & \multirow[b]{3}{*}{ Comment $^{\mathrm{a}}$} & \multirow[b]{3}{*}{ Sample } \\
\hline & & & & & & & & & & & & \\
\hline & & & To Water & Well & & & & Tetrachloride & Chloroform & Chloride & & \\
\hline MW6S & $10-25$ & 03/28/12 & 6.38 & 26.9 & 7 & L & 17.5 & ND & ND & ND & LF & MRMW6S-W-30108 \\
\hline MW6S & $10-25$ & $05 / 07 / 13$ & 6.3 & 26.9 & 6.5 & L & 17.5 & ND & ND & ND & LF & MRMW6S-W-30145 \\
\hline MW6S & $10-25$ & $06 / 05 / 14$ & 6.54 & 26.9 & 9.25 & L & 16.01 & ND & ND & ND & LF & MRMW6S-W-31950 \\
\hline MW6S & $10-25$ & $04 / 29 / 15$ & 6.6 & 26.9 & 9 & L & 17.5 & ND & ND & ND & LF & MRMW6S-W-31954 \\
\hline MW6S & $10-25$ & 05/07/16 & 5.36 & 26.9 & 7.75 & L & 17.5 & ND & ND & ND & LF & MRMW6S-W-39172 \\
\hline MW6S & $10-25$ & $06 / 02 / 17$ & 5.93 & 26.9 & 6.5 & L & 17.5 & ND & ND & ND & LF & MRMW6S-W-39795 \\
\hline MW7S & $20-45$ & 06/03/04 & 26.68 & 46.98 & 40 & gal & - & 18 & ND & ND & TWV & MRMW7S-W-16466 \\
\hline MW7S & $20-45$ & $09 / 12 / 05$ & 17.57 & 46.94 & 55 & gal & - & 43 & 1.1 & ND & TWV & MRMW7S-W-19258 \\
\hline MW7S & $20-45$ & $03 / 22 / 06$ & 22.45 & 46.98 & 48 & gal & - & 21 & $0.4 \mathrm{~J}$ & ND & TWV & MRMW7S-W-20000 \\
\hline MW7S & $20-45$ & 09/19/06 & 20.94 & 47.02 & 56 & gal & - & 38 & $0.7 \mathrm{~J}$ & ND & TWV & MRMW7S-W-22490 \\
\hline MW7S & $20-45$ & $03 / 20 / 07$ & 18.01 & 47.02 & 50 & gal & - & 16 & $0.4 \mathrm{~J}$ & ND & TWV & MRMW7S-W-16481 \\
\hline MW7S & $20-45$ & $10 / 01 / 07$ & 12.40 & 47 & 70 & gal & - & 8.1 & $0.2 \mathrm{~J}$ & ND & TWV & MRMW7S-W-16581 \\
\hline MW7S & $20-45$ & $04 / 14 / 08$ & 7.72 & 47 & 1.82 & $\mathrm{~L}$ & - & 10 & $0.3 \mathrm{~J}$ & ND & $\mathrm{LF}$ & MRMW7S-W-23237 \\
\hline MW7S & $20-45$ & $04 / 23 / 08$ & 7.80 & 47 & 11 & L & - & 8.3 & $0.2 \mathrm{~J}$ & ND & LF & MRMW7S-W-23265 \\
\hline MW7S & $20-45$ & $10 / 20 / 08$ & 17.20 & 47 & 6.3 & L & 32.5 & 7.9 & ND & ND & LF & MRMW7S-W-27627 \\
\hline MW7S & $20-45$ & $04 / 23 / 09$ & 16.70 & 47 & 7 & L & 32.5 & 9.5 & ND & ND & LF & MRMW7S-W-27659 \\
\hline MW7S & $20-45$ & 09/03/09 & 13.80 & 47 & 9 & $\mathrm{~L}$ & 32.5 & 8.0 & ND & ND & LF & MRMW7S-W-29949 \\
\hline MW7S & $20-45$ & $04 / 06 / 10$ & 6.38 & 47 & 5.4 & L & 32.5 & 15 & $0.4 \mathrm{~J}$ & ND & LF & MRMW7S-W-29989 \\
\hline MW7S & $20-45$ & 09/23/10 & 14.15 & 45 & 6 & $\mathrm{~L}$ & 32.5 & 6.6 & ND & ND & LF & MRMW7S-W-30017 \\
\hline MW7S & $20-45$ & $04 / 20 / 11$ & 19.69 & 46.69 & 6 & $\bar{L}$ & 32.5 & 4.4 & $0.2 \mathrm{~J}$ & ND & $\mathrm{LF}$ & MRMW7S-W-30045 \\
\hline MW7S & $20-45$ & $10 / 04 / 11$ & 7.11 & 47 & 7 & $\mathrm{~L}$ & 32.5 & 11 & $0.3 \mathrm{~J}$ & ND & LF & MRMW7S-W-30074 \\
\hline MW7S & $20-45$ & $03 / 28 / 12$ & 15.35 & 47 & 8 & $\mathrm{~L}$ & 32.5 & 12 & $0.3 \mathrm{~J}$ & ND & $\mathrm{LF}$ & MRMW7S-W-30109 \\
\hline MW7S & $20-45$ & $05 / 07 / 13$ & 19.4 & 47 & 6.25 & L & 32.5 & 4.2 & $0.2 \mathrm{~J}$ & ND & LF & MRMW7S-W-30146 \\
\hline MW7S & $20-45$ & $06 / 06 / 14$ & 21.65 & 47 & 7.5 & $\bar{L}$ & 33.75 & 2.3 & ND & ND & $\mathrm{LF}$ & MRMW7S-W-31951 \\
\hline MW7S & $20-45$ & $04 / 29 / 15$ & 23.4 & 47 & 6 & $\mathrm{~L}$ & 34.53 & 2.3 & 1.5 & ND & LF & MRMW7S-W-37555 \\
\hline MW7S & $20-45$ & 05/06/16 & 10.03 & 47 & 7.5 & $\mathrm{~L}$ & 32.5 & 5.9 & ND & ND & LF & MRMW7S-W-39173 \\
\hline MW7S & $20-45$ & $05 / 31 / 17$ & 10.02 & 47 & 6 & $\mathrm{~L}$ & 32.5 & 5.0 & ND & ND & LF & MRMW7S-W-39796 \\
\hline MW8S & $10-25$ & 06/03/04 & 3.70 & 26.75 & 45 & gal & - & ND & ND & ND & TWV & MRMW8S-W-16464 \\
\hline MW8S & $10-25$ & 09/14/05 & 4.02 & 26.82 & 57 & gal & - & $0.9 \mathrm{~J}$ & ND & ND & TWV & MRMW8S-W-19265 \\
\hline MW8S & $10-25$ & 03/20/06 & 4.57 & 26.41 & 43 & gal & - & $0.6 \mathrm{~J}$ & ND & $0.4 \mathrm{~J} \mathrm{~B}$ & TWV & MRMW8S-W-19991 \\
\hline MW8S & $10-25$ & 09/19/06 & 4.76 & 26.83 & 45 & gal & - & 1.3 & ND & ND & TWV & MRMW8S-W-22492 \\
\hline MW8S & $10-25$ & $03 / 20 / 07$ & 2.63 & 26.82 & 49 & gal & _- & $0.6 \mathrm{~J}$ & ND & ND & TWV & MRMW8S-W-16483 \\
\hline MW8S & $10-25$ & $10 / 02 / 07$ & 2.20 & 26.8 & 48 & gal & - & $0.8 \mathrm{~J}$ & ND & ND & TWV & MRMW8S-W-16584 \\
\hline MW8S & $10-25$ & $04 / 15 / 08$ & 0.70 & 26.8 & 5.5 & $\mathrm{~L}$ & - & 1.1 & ND & ND & LF & MRMW8S-W-23238 \\
\hline MW8S & $10-25$ & $10 / 20 / 08$ & 3.60 & 26.8 & 8 & $\mathrm{~L}$ & 17.5 & 1.3 & ND & ND & LF & MRMW8S-W-27628 \\
\hline MW8S & $10-25$ & $04 / 23 / 09$ & 2.30 & 26.8 & 6 & L & 17.5 & ND & ND & ND & $\mathrm{LF}$ & MRMW8S-W-27660 \\
\hline MW8S & $10-25$ & 09/03/09 & 2.90 & 26.8 & 8.5 & $\mathrm{~L}$ & 17.5 & 1.9 & ND & ND & LF & MRMW8S-W-29950 \\
\hline MW8S & $10-25$ & $04 / 06 / 10$ & 1.10 & 26.8 & 8 & $\mathrm{~L}$ & 17.5 & 1.7 & ND & ND & LF & MRMW8S-W-29990 \\
\hline MW8S & $10-25$ & $09 / 22 / 10$ & 2.53 & 26.8 & 9 & L & 17.5 & 1.6 & ND & ND & LF & MRMW8S-W-30018 \\
\hline
\end{tabular}


TABLE 3.2 (Cont.)

\begin{tabular}{|c|c|c|c|c|c|c|c|c|c|c|c|c|}
\hline \multirow{2}{*}{ Location } & \multirow{2}{*}{$\begin{array}{l}\text { Screen } \\
\text { Interval } \\
\text { (ft BGL) }\end{array}$} & \multirow[b]{2}{*}{$\begin{array}{c}\text { Sample } \\
\text { Date }\end{array}$} & \multicolumn{2}{|c|}{ Depth (ft TOC) } & \multirow[b]{2}{*}{$\begin{array}{l}\text { Volume } \\
\text { Purged }\end{array}$} & \multirow[b]{2}{*}{$\begin{array}{l}\text { Purge } \\
\text { Units }\end{array}$} & \multirow{2}{*}{$\begin{array}{l}\text { Pump } \\
\text { Intake } \\
\text { Position } \\
\text { (ft BGL) }\end{array}$} & \multicolumn{3}{|c|}{ Concentration $(\mu \mathrm{g} / \mathrm{L})$} & \multirow[b]{2}{*}{ Comment $^{\mathrm{a}}$} & \multirow[b]{2}{*}{ Sample } \\
\hline & & & To Water & Well & & & & $\begin{array}{c}\text { Carbon } \\
\text { Tetrachloride }\end{array}$ & Chloroform & $\begin{array}{l}\text { Methylene } \\
\text { Chloride }\end{array}$ & & \\
\hline MW8S & $10-25$ & $04 / 20 / 11$ & 2.73 & 26.8 & 9 & $\mathrm{~L}$ & 17.5 & $0.2 \mathrm{~J}$ & ND & ND & LF & MRMW8S-W-30046 \\
\hline MW8S & $10-25$ & $10 / 04 / 11$ & 4.91 & 26.3 & 9 & L & 17.5 & $0.6 \mathrm{~J}$ & ND & ND & LF & MRMW8S-W-30075 \\
\hline MW8S & $10-25$ & $03 / 28 / 12$ & 1.38 & 26.8 & 6.25 & $\mathrm{~L}$ & 17 & $0.4 \mathrm{~J}$ & ND & ND & LF & MRMW8S-W-30110 \\
\hline MW8S & $10-25$ & $05 / 07 / 13$ & 1.42 & 26.8 & 7 & L & 17.5 & $0.9 \mathrm{~J}$ & ND & ND & LF & MRMW8S-W-30147 \\
\hline MW8S & $10-25$ & $06 / 05 / 14$ & 1.89 & 26.8 & 11 & $\mathrm{~L}$ & 17.5 & $0.5 \mathrm{~J}$ & ND & ND & LF & MRMW8S-W-31952 \\
\hline MW8S & $10-25$ & $04 / 29 / 15$ & 4.7 & 26.8 & 9 & L & 17.5 & $0.9 \mathrm{~J}$ & ND & ND & LF & MRMW8S-W-37556 \\
\hline MW8S & $10-25$ & $05 / 06 / 16$ & 26.8 & 26.8 & L & L & 17.5 & ND & ND & ND & LF & MRMW8S-W-39174 \\
\hline MW8S & $10-25$ & $05 / 31 / 17$ & 26.8 & 26.8 & L & L & 17.5 & ND & ND & ND & LF & MRMW8S-W-39797 \\
\hline MW9S & $38.83-53.83$ & $03 / 22 / 06$ & 20.20 & 58.62 & 20 & gal & - & ND & ND & ND & TWV & MRMW9S-W-20004 \\
\hline MW9S & $38.83-53.83$ & 09/19/06 & 18.87 & 59 & 22 & gal & - & ND & ND & ND & TWV & MRMW9S-W-22494 \\
\hline MW9S & $38.83-53.83$ & $03 / 20 / 07$ & 16.69 & 59 & 22 & gal & - & ND & ND & ND & TWV & MRMW9S-W-16480 \\
\hline MW9S & $38.83-53.83$ & 10/01/07 & 14.00 & 58.6 & 23 & gal & - & ND & ND & ND & TWV & MRMW9S-W-16582 \\
\hline MW9S & $38.83-53.83$ & $04 / 14 / 08$ & 16.58 & 58.63 & 2.29 & $\mathrm{~L}$ & - & $0.8 \mathrm{~J}$ & ND & ND & LF & MRMW9S-W-23239 \\
\hline MW9S & $38.83-53.83$ & $10 / 20 / 08$ & 21.50 & 58.5 & 11 & $\mathrm{~L}$ & 46.33 & 1.1 & ND & ND & LF & MRMW9S-W-27629 \\
\hline MW9S & $38.83-53.83$ & $04 / 23 / 09$ & 21.90 & 58.5 & 5.5 & $\mathrm{~L}$ & 46.33 & 1.0 & ND & ND & LF & MRMW9S-W-27661 \\
\hline MW9S & $38.83-53.83$ & 09/04/09 & 19.95 & 58.5 & 5 & $\mathrm{~L}$ & 46.33 & 1.4 & ND & ND & LF & MRMW9S-W-29951 \\
\hline MW9S & $38.83-53.83$ & $04 / 06 / 10$ & 16.50 & 58.5 & 6 & $\mathrm{~L}$ & 46.33 & 1.9 & ND & ND & LF & MRMW9S-W-29991 \\
\hline MW9S & $38.83-53.83$ & $09 / 22 / 10$ & 21.15 & 58.83 & 7 & L & 46.33 & 1.9 & ND & ND & LF & MRMW9S-W-30019 \\
\hline MW9S & $38.83-53.83$ & $04 / 20 / 11$ & 23.76 & 58.32 & 14 & $\mathrm{~L}$ & 46.33 & $0.9 \mathrm{~J}$ & ND & ND & LF & MRMW9S-W-30047 \\
\hline MW9S & $38.83-53.83$ & $10 / 04 / 11$ & 22.35 & 58.5 & 8 & L & 46.33 & $0.9 \mathrm{~J}$ & ND & ND & LF & MRMW9S-W-30076 \\
\hline MW9S & $38.83-53.83$ & $03 / 28 / 12$ & 23.19 & 58.5 & 6.25 & $\mathrm{~L}$ & 46.33 & 1.3 & ND & ND & LF & MRMW9S-W-30111 \\
\hline MW9S & $38.83-53.83$ & $05 / 07 / 13$ & 23.48 & 58.5 & 7 & L & 46.33 & 1.1 & ND & ND & LF & MRMW9S-W-30148 \\
\hline MW9S & $38.83-53.83$ & $06 / 06 / 14$ & 25.51 & 58.5 & 8 & $\mathrm{~L}$ & 46.33 & 1.1 & ND & ND & LF & MRMW9S-W-31953 \\
\hline MW9S & $38.83-53.83$ & $04 / 29 / 15$ & 25.7 & 58.5 & 6 & L & 46.33 & 1.0 & $0.5 \mathrm{~J}$ & ND & LF & MRMW9S-W-37557 \\
\hline MW9S & $38.83-53.83$ & 05/06/16 & 19.00 & 58.5 & 7.25 & $\mathrm{~L}$ & 46.33 & 1.0 & ND & ND & LF & MRMW9S-W-39175 \\
\hline MW9S & $38.83-53.83$ & $05 / 31 / 17$ & 18.65 & 58.8 & 5.5 & L & 46.33 & ND & ND & ND & LF & MRMW9S-W-39798 \\
\hline MW10S & $30-45$ & 03/21/06 & 12.30 & 49.6 & 19 & gal & - & ND & ND & ND & TWV & MRMW10S-W-19999 \\
\hline MW10S & $30-45$ & 09/18/06 & 11.08 & 49.61 & 20 & gal & - & ND & ND & ND & TWV & MRMW10S-W-22489 \\
\hline MW10S & $30-45$ & $03 / 21 / 07$ & 10.77 & 49.61 & 20 & gal & - & ND & ND & ND & TWV & MRMW10S-W-16485 \\
\hline MW10S & $30-45$ & $10 / 01 / 07$ & 6.95 & 49.65 & 20 & gal & - & ND & ND & ND & TWV & MRMW10S-W-16593 \\
\hline MW10S & $30-45$ & $04 / 14 / 08$ & 9.82 & 49.7 & 1.9 & $\mathrm{~L}$ & - & ND & ND & ND & LF & MRMW10S-W-23240 \\
\hline MW10S & $30-45$ & $10 / 20 / 08$ & 13.70 & 49.7 & 5.4 & $\mathrm{~L}$ & 37.5 & ND & ND & ND & LF & MRMW10S-W-27630 \\
\hline MW10S & $30-45$ & $04 / 23 / 09$ & 13.60 & 45 & 7.5 & $\mathrm{~L}$ & 37.5 & ND & ND & ND & LF & MRMW10S-W-27662 \\
\hline MW10S & $30-45$ & 09/03/09 & 12.00 & 49.7 & 7.5 & L & 37.5 & ND & ND & ND & LF & MRMW10S-W-29952 \\
\hline MW10S & $30-45$ & $04 / 06 / 10$ & 7.50 & 49.7 & 9 & $\mathrm{~L}$ & 37.5 & ND & ND & ND & LF & MRMW10S-W-29992 \\
\hline MW10S & $30-45$ & $09 / 22 / 10$ & 11.69 & 49.7 & 11 & L & 37.5 & ND & $0.3 \mathrm{~J}$ & ND & LF & MRMW10S-W-30020 \\
\hline MW10S & $30-45$ & $04 / 20 / 11$ & 13.69 & 49.3 & 7.25 & $\mathrm{~L}$ & 37.5 & ND & $0.2 \mathrm{~J}$ & ND & LF & MRMW10S-W-30048 \\
\hline MW10S & $30-45$ & $10 / 04 / 11$ & 12.54 & 49.7 & 8 & L & 37.5 & ND & ND & ND & LF & MRMW10S-W-30077 \\
\hline MW10S & $30-45$ & $03 / 28 / 12$ & 12.76 & 49.7 & 8 & L & 37.5 & ND & $0.3 \mathrm{~J}$ & ND & LF & MRMW10S-W-30112 \\
\hline
\end{tabular}


TABLE 3.2 (Cont.)

\begin{tabular}{|c|c|c|c|c|c|c|c|c|c|c|c|c|}
\hline \multirow[b]{2}{*}{ Location } & \multirow{2}{*}{$\begin{array}{l}\text { Screen } \\
\text { Interval } \\
\text { (ft BGL) }\end{array}$} & \multirow[b]{2}{*}{$\begin{array}{l}\text { Sample } \\
\text { Date }\end{array}$} & \multicolumn{2}{|c|}{ Depth (ft TOC) } & \multirow[b]{2}{*}{$\begin{array}{l}\text { Volume } \\
\text { Purged }\end{array}$} & \multirow[b]{2}{*}{$\begin{array}{l}\text { Purge } \\
\text { Units }\end{array}$} & \multirow{2}{*}{$\begin{array}{l}\text { Pump } \\
\text { Intake } \\
\text { Position } \\
\text { (ft BGL) }\end{array}$} & \multicolumn{3}{|c|}{ Concentration $(\mu \mathrm{g} / \mathrm{L})$} & \multirow[b]{2}{*}{ Comment $^{\mathrm{a}}$} & \multirow[b]{2}{*}{ Sample } \\
\hline & & & To Water & Well & & & & $\begin{array}{c}\text { Carbon } \\
\text { Tetrachloride }\end{array}$ & Chloroform & $\begin{array}{l}\text { Methylene } \\
\text { Chloride }\end{array}$ & & \\
\hline MW10S & $30-45$ & 05/07/13 & 13.78 & 49.7 & 6 & L & 37.5 & ND & ND & ND & LF & MRMW10S-W-30149 \\
\hline MW10S & $30-45$ & $06 / 05 / 14$ & 15.89 & 49.7 & 7 & $\mathrm{~L}$ & 37.5 & ND & $0.7 \mathrm{~J}$ & ND & LF & MRMW10S-W-31954 \\
\hline MW10S & $30-45$ & $04 / 29 / 15$ & 16.35 & 49.7 & 7 & $\mathrm{~L}$ & 37.5 & ND & 1.1 & ND & LF & MRMW10S-W-37558 \\
\hline MW10Si & $30-45$ & 05/07/16 & 8.25 & 49.7 & 7.75 & L & 37.5 & ND & 2.1 & ND & LF & MRMW10S-W-39176 \\
\hline MW11S & $53-68$ & $03 / 22 / 06$ & 35.20 & 72.5 & 20 & gal & - & 39 & $0.9 \mathrm{~J}$ & ND & TWV & MRMW11S-W-20001 \\
\hline MW11S & $53-68$ & 09/19/06 & 36.00 & 73.14 & 20 & gal & - & 53 & 1.0 & ND & TWV & MRMW11S-W-22491 \\
\hline MW11S & $53-68$ & 03/20/07 & 34.65 & 73.14 & 20 & gal & - & 37 & $0.8 \mathrm{~J}$ & ND & TWV & MRMW11S-W-16479 \\
\hline MW11S & $53-68$ & $10 / 01 / 07$ & 31.55 & 73 & 20 & gal & - & 54 & 1.2 & ND & TWV & MRMW11S-W-16594 \\
\hline MW11S & $53-68$ & 04/15/08 & 29.90 & 72.7 & 5.5 & $\mathrm{~L}$ & - & 35 & $0.8 \mathrm{~J}$ & ND & LF & MRMW11S-W-23241 \\
\hline MW11S & $53-68$ & $04 / 22 / 08$ & 30.20 & 72.7 & 7.2 & L & - & 42 & $0.9 \mathrm{~J}$ & ND & LF & MRMW11S-W-23261 \\
\hline MW11S & $53-68$ & 10/20/08 & 37.10 & 72.7 & 9 & $\mathrm{~L}$ & 60.5 & 42 & $0.9 \mathrm{~J}$ & ND & LF & MRMW11S-W-27631 \\
\hline MW11S & $53-68$ & $10 / 21 / 08$ & - & - & - & - & - & 45 & $0.9 \mathrm{~J}$ & ND & TWV & MRMW11S-W-27651 \\
\hline MW11S & $53-68$ & $04 / 23 / 09$ & 38.10 & 72.7 & 5 & $\mathrm{~L}$ & 60.5 & 46 & 1.0 & ND & LF & MRMW11S-W-27663 \\
\hline MW11S & $53-68$ & 09/03/09 & 34.70 & 72.7 & 7.5 & $\mathrm{~L}$ & 60.5 & 39 & $0.9 \mathrm{~J}$ & ND & LF & MRMW11S-W-29953 \\
\hline MW11S & $53-68$ & 09/04/09 & 34.95 & 72.7 & 72 & L & 67 & 41 & $0.9 \mathrm{~J}$ & ND & TWV & MRMW11S-W-29973 \\
\hline MW11S & $53-68$ & $04 / 06 / 10$ & 29.45 & 72.7 & 6.5 & $\mathrm{~L}$ & 60.5 & 38 & 1.0 & ND & LF & MRMW11S-W-29993 \\
\hline MW11S & $53-68$ & $09 / 23 / 10$ & 34.75 & 72.7 & 7 & $\mathrm{~L}$ & 60.5 & 28 & 1.0 & ND & LF & MRMW11S-W-30021 \\
\hline MW11S & $53-68$ & $04 / 20 / 11$ & 39.16 & 72.5 & 7.5 & L & 60.5 & 38 & 1.1 & ND & LF & MRMW11S-W-30049 \\
\hline MW11S & $53-68$ & $10 / 04 / 11$ & 36.83 & 72.7 & 6 & $\mathrm{~L}$ & 60.5 & 33 & $0.8 \mathrm{~J}$ & ND & LF & MRMW11S-W-30078 \\
\hline MW11S & $53-68$ & 03/28/12 & 36.46 & 72.7 & 6 & $\mathrm{~L}$ & 60.5 & 39 & 1.1 & ND & LF & MRMW11S-W-30113 \\
\hline MW11S & $53-68$ & $05 / 07 / 13$ & 38.46 & 72.7 & 6 & $\mathrm{~L}$ & 60.5 & 37 & ND & ND & LF & MRMW11S-W-30150 \\
\hline MW11S & $53-68$ & $06 / 05 / 14$ & 41.21 & 72.7 & 7 & $\mathrm{~L}$ & 60.5 & 28 & $0.7 \mathrm{~J}$ & ND & LF & MRMW11S-W-31955 \\
\hline MW11S & $53-68$ & $05 / 06 / 15$ & 40.90 & 72.7 & 6 & $\mathrm{~L}$ & 60.5 & 22 & $0.6 \mathrm{~J}$ & ND & LF & MRMW11S-W-37559 \\
\hline MW11S & $53-68$ & 05/06/16 & 29.75 & 72.7 & 8 & $\mathrm{~L}$ & 60.5 & 22 & $0.6 \mathrm{~J}$ & ND & LF & MRMW11S-W-39177 \\
\hline MW11S & $53-68$ & $06 / 02 / 17$ & 30.06 & 72.7 & 5.5 & L & 60.5 & 25 & ND & ND & LF & MRMW11S-W-39800 \\
\hline Isch & - & 02/19/04 & - & - & - & - & - & ND & ND & ND & PUMP & MRJR-W-16502 \\
\hline Isch & - & $09 / 14 / 05$ & - & - & - & - & - & ND & ND & ND & PUMP & MRPRISCH-W-16513 \\
\hline Isch & - & 03/23/06 & - & - & 20 & gal & - & ND & ND & ND & PUMP & MRISCH-W-19989 \\
\hline Isch & - & 09/19/06 & - & - & - & - & - & ND & ND & ND & PUMP & MRISCH-W-16531 \\
\hline Isch & - & $03 / 22 / 07$ & - & - & - & - & - & ND & ND & ND & PUMP & MRISCH-W-16564 \\
\hline Isch & - & $10 / 03 / 07$ & - & - & - & - & - & ND & ND & ND & PUMP & MRISCH-W-16590 \\
\hline Isch & - & 04/15/08 & - & - & - & - & - & $0.4 \mathrm{~J}$ & ND & ND & PUMP & MRISCH-W-23242 \\
\hline Isch & - & $04 / 22 / 08$ & - & - & - & - & - & ND & ND & ND & PUMP & MRISCH-W-23262 \\
\hline Isch & - & 10/21/08 & - & - & - & - & - & ND & ND & ND & PUMP & MRISCH-W-27632 \\
\hline Isch & - & $04 / 22 / 09$ & - & - & - & - & - & ND & ND & ND & PUMP & MRISCH-W-27664 \\
\hline Isch & - & 09/02/09 & - & - & - & - & - & ND & ND & ND & PUMP & MRISCH-W-29954 \\
\hline Isch & - & $04 / 07 / 10$ & - & - & - & - & - & ND & ND & ND & PUMP & MRISCH-W-29994 \\
\hline Isch & - & $09 / 22 / 10$ & - & - & - & - & - & ND & ND & ND & PUMP & MRISCH-W-30022 \\
\hline Isch & - & $04 / 21 / 11$ & - & - & - & - & - & ND & ND & ND & PUMP & MRISCH-W-30050 \\
\hline
\end{tabular}


TABLE 3.2 (Cont.)

\begin{tabular}{|c|c|c|c|c|c|c|c|c|c|c|c|c|}
\hline \multirow[b]{3}{*}{ Location } & \multirow{3}{*}{$\begin{array}{l}\text { Screen } \\
\text { Interval } \\
\text { (ft BGL) }\end{array}$} & \multirow{3}{*}{$\begin{array}{l}\text { Sample } \\
\text { Date }\end{array}$} & \multirow{2}{*}{\multicolumn{2}{|c|}{ Depth (ft TOC) }} & \multirow{3}{*}{$\begin{array}{l}\text { Volume } \\
\text { Purged }\end{array}$} & \multirow{3}{*}{$\begin{array}{l}\text { Purge } \\
\text { Units }\end{array}$} & \multirow{3}{*}{$\begin{array}{l}\text { Pump } \\
\text { Intake } \\
\text { Position } \\
\text { (ft BGL) }\end{array}$} & \multicolumn{3}{|c|}{ Concentration $(\mu \mathrm{g} / \mathrm{L})$} & \multirow[b]{3}{*}{ Comment $^{a}$} & \multirow[b]{3}{*}{ Sample } \\
\hline & & & & & & & & & & & & \\
\hline & & & To Water & Well & & & & Tetrachloride & Chloroform & Chloride & & \\
\hline Isch & - & $10 / 03 / 11$ & - & - & - & - & - & ND & ND & ND & PUMP & MRIsch-W-30079 \\
\hline Isch & - & 03/28/12 & - & - & - & - & - & ND & ND & ND & PUMP & MRISCH-W-30114 \\
\hline Isch & - & 05/07/13 & - & - & - & - & - & ND & ND & ND & PUMP & MRISCH-W-30151 \\
\hline Isch & - & 06/06/14 & - & - & - & - & - & ND & ND & ND & PUMP & MRISCH-W-31956 \\
\hline $\operatorname{Isch}^{\mathrm{j}}$ & - & $04 / 30 / 15$ & - & - & - & - & - & ND & ND & ND & PUMP & MRISCH-W-31960 \\
\hline Rilinger & - & 06/04/04 & - & - & - & - & - & ND & ND & ND & PUMP & MRPRIVRIL-W-16471 \\
\hline Rilinger & - & 09/14/05 & - & - & - & - & - & 2.6 & $0.1 \mathrm{~J}$ & ND & PUMP & MRPRILL-W-16512 \\
\hline Rilinger & - & 03/19/06 & - & - & - & - & - & ND & ND & $0.4 \mathrm{~J} \mathrm{~B}$ & PUMP & MRRILINGER-W-19988 \\
\hline Rilinger & - & 09/19/06 & - & - & - & - & - & ND & ND & ND & PUMP & MRRILI-W-16530 \\
\hline Rilinger & - & 03/29/07 & - & - & - & - & - & 1.3 & 1.1 & ND & PUMP & MRRILINGER-W-16561 \\
\hline Rilinger & - & $10 / 03 / 07$ & - & - & - & - & - & $13^{f}$ & $0.4 \mathrm{~J}$ & ND & PUMP & MRRILINGER-W-16591 \\
\hline Rilinger & - & $10 / 08 / 07$ & - & - & - & - & - & $0.4 \mathrm{~J}$ & ND & ND & PUMP & MRRILINGER-W-16592 \\
\hline Rilinger & - & 01/11/08 & - & - & - & - & - & 6.2 & $0.5 \mathrm{~J}$ & ND & PUMP & MORIL-W-11108 \\
\hline Rilinger & - & 04/15/08 & - & - & - & - & - & 9.9 & $0.4 \mathrm{~J}$ & ND & PUMP & MRRILINGER-W-23243 \\
\hline Rilinger & - & 10/21/08 & - & - & - & - & - & $0.9 \mathrm{~J}$ & ND & ND & PUMP & MRRILLINGER-W-27633 \\
\hline Rilinger & - & $04 / 22 / 09$ & - & - & - & - & - & 1.2 & ND & ND & PUMP & MRRILLINGER-W-27665 \\
\hline Rilinger & - & 09/02/09 & - & - & - & - & - & 1.0 & ND & ND & PUMP & MRRILLINGER-W-29955 \\
\hline Rilinger & - & 04/07/10 & - & - & - & - & - & $0.8 \mathrm{~J}$ & ND & ND & PUMP & MRRILLINGER-W-29995 \\
\hline Rilinger & - & 09/22/10 & - & - & - & - & - & 1.3 & ND & ND & PUMP & MRRILLINGER-W-30023 \\
\hline Rilinger & - & $04 / 21 / 11$ & - & - & - & - & - & $0.7 \mathrm{~J}$ & ND & ND & PUMP & MRRILLINGER-W-30051 \\
\hline Rilinger & - & 10/03/11 & - & - & - & - & - & $0.7 \mathrm{~J}$ & ND & ND & PUMP & MRRilinger-W-30080 \\
\hline Rilinger & - & 03/28/12 & - & - & - & - & - & $0.7 \mathrm{~J}$ & ND & ND & PUMP & MRRILLINGER-W-30115 \\
\hline Rilinger & - & $05 / 07 / 13$ & - & - & - & - & - & 2.5 & ND & ND & PUMP & MRRILLINGER-W-30152 \\
\hline Rilinger & - & $06 / 06 / 14$ & - & - & - & - & - & 8.0 & $0.3 \mathrm{~J}$ & ND & PUMP & MRRILLINGER-W-31957 \\
\hline Rilinger & - & 04/29/15 & - & - & - & - & - & 1.7 & ND & ND & PUMP & MRRILLINGER-W-37561 \\
\hline Rilinger & - & 05/08/16 & - & - & - & - & - & ND & ND & ND & PUMP & MRRILLINGER-W-39178 \\
\hline Rilinger & - & $06 / 01 / 17$ & - & - & - & - & - & $0.6 \mathrm{~J}$ & ND & ND & PUMP & MRRILLINGER-W-39801 \\
\hline Stone & $43^{g}$ & 06/04/04 & 23.35 & - & - & & - & 10 & ND & ND & PDS & MRPRIVSTON-W-16475 \\
\hline Stone & 43 & 09/14/05 & 17.18 & 40 & - & - & - & 2.6 & $0.3 \mathrm{~J}$ & ND & TWV & MRPRSTON-W-16511 \\
\hline Stone & 43 & 03/19/06 & 17.42 & 40 & 100 & gal & - & 14 & $0.8 \mathrm{~J}$ & $0.4 \mathrm{~J} \mathrm{~B}$ & TWV & MRSTONE-W-19987 \\
\hline Stone & 43 & 09/19/06 & 18.55 & 38.8 & 41 & gal & - & 2.1 & ND & ND & TWV & MRSTONE-W-16529 \\
\hline Stone & 43 & 03/22/07 & 20.62 & 38.8 & 56 & gal & - & 5.4 & $0.3 \mathrm{~J}$ & ND & TWV & MRSTONE-W-16560 \\
\hline Stone & 43 & $10 / 03 / 07$ & 14.60 & 38.6 & 72 & gal & - & 2.8 & ND & ND & TWV & MRSTONE-W-16589 \\
\hline Stone & 43 & 04/15/08 & - & 38.86 & - & - & - & $0.9 \mathrm{~J}$ & ND & ND & BAIL & MRSTONE-W-23244 \\
\hline Stone & 43 & 10/21/08 & - & - & 5 & gal & - & 3.0 & ND & ND & BAIL & MRSTONE-W-27634 \\
\hline Stone & 43 & 04/23/09 & - & - & 5 & gal & - & 1.1 & ND & ND & BAIL & MRSTONE-W-27666 \\
\hline Stone & 43 & 09/02/09 & - & - & 5 & gal & - & $0.9 \mathrm{~J}$ & ND & ND & BAIL & MRSTONE-W-29956 \\
\hline Stone & 43 & 04/07/10 & - & - & 5 & gal & - & $0.5 \mathrm{~J}$ & ND & ND & BAIL & MRSTONE-W-29996 \\
\hline Stone & 43 & $09 / 22 / 10$ & - & - & 5 & gal & - & $0.6 \mathrm{~J}$ & 1.8 & ND & BAIL & MRSTONE-W-30024 \\
\hline
\end{tabular}


TABLE 3.2 (Cont.)

\begin{tabular}{|c|c|c|c|c|c|c|c|c|c|c|c|c|}
\hline \multirow[b]{2}{*}{ Location } & \multirow{2}{*}{$\begin{array}{l}\text { Screen } \\
\text { Interval } \\
\text { (ft BGL) }\end{array}$} & \multirow[b]{2}{*}{$\begin{array}{l}\text { Sample } \\
\text { Date }\end{array}$} & \multicolumn{2}{|c|}{ Depth (ft TOC) } & \multirow[b]{2}{*}{$\begin{array}{l}\text { Volume } \\
\text { Purged }\end{array}$} & \multirow[b]{2}{*}{$\begin{array}{l}\text { Purge } \\
\text { Units }\end{array}$} & \multirow{2}{*}{$\begin{array}{l}\text { Pump } \\
\text { Intake } \\
\text { Position } \\
\text { (ft BGL) }\end{array}$} & \multicolumn{3}{|c|}{ Concentration $(\mu \mathrm{g} / \mathrm{L})$} & \multirow[b]{2}{*}{ Comment $^{a}$} & \multirow[b]{2}{*}{ Sample } \\
\hline & & & To Water & Well & & & & $\begin{array}{c}\text { Carbon } \\
\text { Tetrachloride }\end{array}$ & Chloroform & $\begin{array}{l}\text { Methylene } \\
\text { Chloride }\end{array}$ & & \\
\hline Stone & 43 & $04 / 21 / 11$ & - & - & 5 & gal & - & 1.8 & $0.9 \mathrm{~J}$ & ND & BAIL & MRSTONE-W-30052 \\
\hline Stone & 43 & $10 / 03 / 11$ & - & - & 5 & gal & - & 1.5 & ND & ND & BAIL & MRStone-W-30081 \\
\hline Stone & 43 & 03/28/12 & - & - & 5 & gal & - & $0.5 \mathrm{~J}$ & ND & ND & BAIL & MRSTONE-W-30116 \\
\hline Stone & 43 & $05 / 07 / 13$ & - & - & 5 & gal & - & $0.4 \mathrm{~J}$ & ND & ND & BAIL & MRSTONE-W-30153 \\
\hline Stone & 43 & 06/06/14 & 17.65 & - & 5 & gal & - & ND & ND & ND & BAIL & MRSTONE-W-31958 \\
\hline Stone & 43 & 05/06/15 & - & - & 5 & gal & - & $0.9 \mathrm{~J}$ & ND & ND & BAIL & MRSTONE-W-37562 \\
\hline Stone & 43 & 05/08/16 & - & - & 5 & gal & - & ND & ND & ND & BAIL & MRSTONE-W-39179 \\
\hline Stone & 43 & $06 / 01 / 17$ & - & - & 5 & gal & - & 1.2 & ND & ND & BAIL & MRSTONE-W-39802 \\
\hline TD12 & $27-67$ & $04 / 22 / 08$ & - & - & - & - & - & ND & ND & ND & GRIMM & MRTD12-W-23264 \\
\hline TD12 & $27-67$ & 10/20/08 & - & - & - & - & - & ND & ND & ND & GRIMM & MRTD12-W-27635 \\
\hline TD12 & $27-67$ & $04 / 23 / 09$ & - & - & - & - & - & ND & ND & ND & TD & MRTD12-W-27667 \\
\hline TD12 & $27-67$ & 09/03/09 & - & - & - & - & - & ND & ND & ND & OVER & MRTD12-W-29957 \\
\hline TD12 & $27-67$ & $04 / 06 / 10$ & - & - & - & - & - & ND & ND & ND & OVER & MRTD12-W-29997 \\
\hline TD12 & $27-67$ & $09 / 22 / 10$ & - & - & - & - & - & ND & ND & ND & OVER & MRTD12-W-30025 \\
\hline TD12 & $27-67$ & $04 / 20 / 11$ & - & - & - & - & - & ND & ND & ND & OVER & MRTD12-W-30053 \\
\hline TD12 & $27-67$ & 10/03/11 & - & - & - & - & - & ND & ND & ND & OVER & MRTD12-W-30082 \\
\hline TD12 & $27-67$ & 03/28/12 & - & - & - & - & - & ND & ND & ND & OVER & MRTD12-W-30117 \\
\hline TD12 & $27-67$ & $05 / 07 / 13$ & - & - & - & - & - & ND & ND & ND & OVER & MRTD12-W-30154 \\
\hline TD12 & $27-67$ & $06 / 06 / 14$ & - & - & - & - & - & ND & ND & ND & OVER & MRTD12-W-31959 \\
\hline $\mathrm{TD}^{\mathrm{j}} 2^{\mathrm{j}}$ & $27-67$ & $04 / 29 / 15$ & - & - & _- & - & _- & ND & ND & ND & OVER & MRTD12-W-37563 \\
\hline
\end{tabular}

Footnotes on following page. 
a Comments: BAIL; purged with bailer; GRIMM, Grimm irrigation well; LF, low flow (sometimes specified as upper -U, middle -M, or base -B); NS, not sampled (insufficient water); OVER, overflow before catchment; PDB, passive diffusion bag; PDS, purged dry and sampled; PUMP, purged with well's dedicated pump; TD, tile drain; TWV, three-well-volume purge.

b ND, not detected at instrument detection limit of $0.1 \mu \mathrm{g} / \mathrm{L}$.

c $\mathrm{J}$, estimated concentration below the method quantitation limit of $1 \mu \mathrm{g} / \mathrm{L}$.

d B, compound present in the associated method blank.

e R, contaminant present in the associated equipment rinsate. Resampling confirmed that the well was free of contamination.

f Sample collected after recent reactivation of well. Well resampled on 1/8/07 and 1/11/08.

g Total depth.

h Collection of samples using both the low-flow method and passive diffusion bag samplers at MW1S was discontinued in 2017 with KDHE approval (KDHE 2017)

i Sampling at MW2S, MW4S, and MW10S was discontinued in 2017 with KDHE approval (KDHE 2016a).

j Sampling at Isch and Grimm (TD12) wells was discontinued in 2017 with KDHE approval (KDHE 2015). 
TABLE 3.3 Field measurements for groundwater samples collected in 2003-2017.

\begin{tabular}{|c|c|c|c|c|c|c|c|c|c|c|c|c|c|c|c|}
\hline Location & $\begin{array}{l}\text { Screen } \\
\text { Interval } \\
\text { (ft BGL) }\end{array}$ & $\begin{array}{l}\text { Sample } \\
\text { Date }\end{array}$ & $\begin{array}{l}\text { Depth to } \\
\text { Water } \\
\text { (ft TOC) }\end{array}$ & $\begin{array}{l}\text { Depth of } \\
\text { Well } \\
\text { (ft TOC) }\end{array}$ & $\begin{array}{l}\text { Volume } \\
\text { Purged }\end{array}$ & $\begin{array}{l}\text { Purge } \\
\text { Units }\end{array}$ & $\begin{array}{l}\text { Pump } \\
\text { Intake } \\
\text { Position } \\
\text { (ft BGL) }\end{array}$ & $\begin{array}{c}\text { Temperature } \\
\left({ }^{\circ} \mathrm{C}\right)\end{array}$ & $\mathrm{pH}$ & $\begin{array}{c}\text { Conductivity } \\
(\mu \mathrm{S} / \mathrm{cm})\end{array}$ & $\begin{array}{l}\text { Dissolved } \\
\text { Oxygen } \\
\text { (mg/L) }\end{array}$ & $\begin{array}{l}\text { ORP } \\
(\mathrm{mV})\end{array}$ & $\begin{array}{l}\text { Iron II } \\
(\mathrm{mg} / \mathrm{L})\end{array}$ & $\begin{array}{l}\text { Carbon } \\
\text { Dioxide } \\
\text { (mg/L) }\end{array}$ & Sample \\
\hline MW1S & $11-51$ & $10 / 23 / 03$ & 30.36 & 54.04 & 70 & gal & - & 14.6 & 7.14 & 933 & $-^{a}$ & 13 & - & - & MRMW1S-W-16422 \\
\hline MW1S & $11-51$ & $06 / 02 / 04$ & 26.97 & 53.94 & 53 & gal & - & 14.4 & 7.16 & 970 & - & - & - & - & MRMW1S-W-16461 \\
\hline MW1S & $11-51$ & 09/13/05 & 24.16 & 53.9 & 57 & gal & - & 15.3 & 6.95 & 1174 & 7.17 & 200 & 0.00 & 55 & MRMW1S-W-19259 \\
\hline MW1S & $11-51$ & $03 / 22 / 06$ & 29 & 53.95 & 48 & gal & - & 15.5 & 7.23 & 927 & 9.94 & 220 & 0.01 & 40 & MRMW1S-W-20008 \\
\hline MW1S & $11-51$ & 09/20/06 & 26.82 & 53.97 & 55 & gal & - & 15.7 & 7.12 & 973 & 7.52 & - & 0.03 & 40 & MRMW1S-W-22495 \\
\hline MW1S & $11-51$ & 03/21/07 & 25.8 & 53.98 & 55 & gal & - & 16.6 & 6.48 & 960 & 5.45 & 88 & 0.00 & 40 & MRMW1S-W-16488 \\
\hline MW1S & $11-51$ & $10 / 01 / 07$ & 21.65 & 53.95 & 63 & gal & - & 16.0 & 6.80 & 886 & 6.79 & 128 & 0.00 & 30 & MRMW1S-W-16595 \\
\hline MW1S & $11-51$ & $04 / 14 / 08$ & 16.2 & 54 & 5.5 & L & - & 13.9 & 7.09 & 1237 & 6.38 & 118 & 0.02 & - & MRMW1S-W-23230 \\
\hline MW1S & $11-51$ & $04 / 22 / 08$ & 16 & 54 & 6.25 & L & - & 15.6 & 6.96 & 1230 & 6.10 & 133 & - & - & MRMW1S-W-23259 \\
\hline MW1S & $11-51$ & 05/01/08 & - & - & 3.24 & L & 22 & 17.1 & 7.11 & 801 & 3.34 & 104 & - & - & MRMW1S-22-W-23275 \\
\hline MW1S & $11-51$ & $05 / 01 / 08$ & - & - & 3.24 & $\mathrm{~L}$ & 27 & 17.0 & 7.10 & 820 & 3.40 & 102 & - & - & MRMW1S-27-W-23276 \\
\hline MW1S & $11-51$ & 05/01/08 & - & - & 4.25 & L & 48 & 16.3 & 7.00 & 1301 & 3.03 & 118 & - & - & MRMW1S-48-W-23277 \\
\hline MW1S & $11-51$ & 10/20/08 & 25.8 & 54 & 6 & L & 31 & 14.0 & 6.84 & 1265 & 5.40 & 103 & 0.00 & - & MRMW1S-W-27620 \\
\hline MW1S & $11-51$ & $10 / 21 / 08$ & - & - & $\mathrm{TWV}^{\mathrm{b}}$ & - & - & 14.8 & 7.02 & 978 & - & - & 0.00 & - & MRMW1S-W-27649 \\
\hline MW1S & $11-51$ & $04 / 24 / 09$ & 24.4 & 54 & 5 & L & 39.2 & 16.2 & 7.00 & 986 & 8.93 & 75 & 0.04 & - & MRMW1S-W-27652 \\
\hline MW1S & $11-51$ & 09/03/09 & 19 & 54 & 8 & L & 35 & 16.0 & 6.94 & 1334 & 7.08 & 28 & 0.04 & - & MRMW1S-W-29942 \\
\hline MW1S & $11-51$ & 09/04/09 & 19.3 & 51.2 & 244 & $\mathrm{~L}$ & 50 & 15.0 & 6.92 & 950 & 7.73 & 61 & - & - & MRMW1S-W-29971 \\
\hline MW1S & $11-51$ & $04 / 07 / 10$ & 11.65 & 51.3 & 7 & L & 16.6 & 9.6 & 7.01 & 695 & 8.66 & 233 & 0.00 & - & MRMW1ST-W-29981 \\
\hline MW1S & $11-51$ & $04 / 07 / 10$ & 11.57 & 51.31 & 6 & L & 31.5 & 11.9 & 6.96 & 918 & 8.42 & 246 & 0.00 & - & MRMW1SM-W-29980 \\
\hline MW1S & $11-51$ & 04/07/10 & 11.65 & 51.31 & 6 & L & 46.3 & 11.7 & 6.95 & 965 & 8.29 & 251 & 0.01 & - & MRMW1SB-W-29979 \\
\hline MW1S & $11-51$ & $04 / 07 / 10$ & 11.48 & 51.3 & 80 & gal & 49 & 14.0 & 7.18 & 687 & 8.86 & 211 & 0.03 & - & MRMW1S3X-W-29982 \\
\hline MW1S & $11-51$ & 09/22/10 & 19.89 & 54 & 10 & $\mathrm{~L}$ & 31 & 17.9 & 7.01 & 1337 & 6.17 & 134 & 0.07 & - & MRMW1S-W-30010 \\
\hline MW1S & $11-51$ & $04 / 20 / 11$ & 23.45 & 54 & 6.5 & $\mathrm{~L}$ & 37 & 13.8 & 6.96 & 866 & 6.39 & 55 & 0.00 & - & MRMW1S-W-30038 \\
\hline MW1S & $11-51$ & 10/04/11 & 21.80 & 54 & 8 & $\mathrm{~L}$ & 34.9 & 15.4 & 7.65 & 1166 & 5.72 & 98 & 0.00 & - & MRMW1S-W-30067 \\
\hline MW1S & $11-51$ & $10 / 12 / 11$ & 22.14 & 54 & 6 & L & 36.57 & 16.3 & 7.24 & 1279 & 5.76 & 323 & - & - & MRMW1S-W-30091 \\
\hline MW1S & $11-51$ & $10 / 12 / 11$ & 22.41 & 54 & 65 & gal & 49 & 16.7 & 7.36 & 905 & 6.18 & 270 & - & - & MRMW1S3X-W-30092 \\
\hline MW1S & $11-51$ & $03 / 29 / 12$ & 20.10 & 54 & 8 & $\mathrm{~L}$ & 25 & 16.0 & 7.00 & 862 & 6.66 & -3.2 & 0.00 & - & MRMW1SU-W-30099 \\
\hline MW1S & $11-51$ & $03 / 29 / 12$ & 20.17 & 54 & 8 & $\bar{L}$ & 35 & 15.6 & 7.00 & 955 & 6.55 & -5.2 & 0.03 & - & MRMW1SM-W-30100 \\
\hline MW1S & $11-51$ & 03/29/12 & 20.17 & 54 & 8 & $\mathrm{~L}$ & 46 & 15.6 & 7.02 & 953 & 6.55 & -2.3 & 0.00 & - & MRMW1SL-W-30101 \\
\hline MW1S & $11-51$ & 03/29/12 & 20.18 & 54 & 68 & gal & 50 & 16.6 & 7.14 & 837 & 6.61 & 12 & 0.01 & - & MRMW1S3X-W-30102 \\
\hline MW1S & $11-51$ & 05/07/13 & 20.94 & 54 & 6 & $\mathrm{~L}$ & 28.46 & 17.2 & 7.13 & 738 & 8.01 & 38 & 0.00 & - & MRMW1SU-W-30136 \\
\hline MW1S & $11-51$ & $05 / 07 / 13$ & 20.94 & 54 & 6 & L & 35.97 & 16.3 & 7.13 & 748 & 7.89 & 37 & 0.11 & - & MRMW1SM-W-30137 \\
\hline MW1S & $11-51$ & $05 / 07 / 13$ & 20.94 & 54 & 6 & L & 43.49 & 14.8 & 7.12 & 749 & 7.83 & 38 & 0.12 & - & MRMW1SL-W-30138 \\
\hline MW1S & $11-51$ & 05/07/13 & 20.94 & 54 & 65 & gal & - & 14.4 & 7.10 & 724 & 7.31 & 202 & 0.01 & - & MRMW1S3X-W-30139 \\
\hline MW1S & $11-51$ & 06/05/14 & 27.38 & 54 & 7 & L & 30.54 & 17.0 & 7.07 & 852 & 7.65 & 189 & 0.01 & - & MRMW1SU-W-31941 \\
\hline MW1S & $11-51$ & 06/05/14 & 27.38 & 54 & 6 & L & 37.38 & 15.9 & 7.07 & 890 & 7.52 & 176 & 0.04 & - & MRMW1SM-W-31942 \\
\hline MW1S & $11-51$ & $06 / 05 / 14$ & 27.38 & 54 & 6 & L & 44.18 & 15.0 & 7.06 & 895 & 7.48 & 171 & 0.03 & - & MRMW1SL-W-31943 \\
\hline MW1S & $11-51$ & $06 / 06 / 14$ & 27.38 & 54 & 65 & gal & 52 & 15.5 & 7.10 & 913 & 7.57 & 120 & 0.03 & - & MRMW1S3X-W-31944 \\
\hline MW1S & $11-51$ & 04/30/15 & 29.6 & 54 & 6 & L & 32.96 & 17.5 & 7.10 & 1009 & 8.23 & 184 & 0.12 & - & MRMW1S3X-W-37545 \\
\hline MW1S & $11-51$ & 04/30/15 & 29.6 & 54 & 6 & L & 38.98 & 17.3 & 7.12 & 1048 & 7.34 & 179 & 0.03 & - & MRMW1S3X-W-37546 \\
\hline MW1S & $11-51$ & 04/30/15 & 29.6 & 54 & 6 & L & 44.99 & 16.2 & 7.12 & 1048 & 7.25 & 181 & 0.08 & - & MRMW1S3X-W-37547 \\
\hline MW1S & $11-51$ & $04 / 30 / 15$ & 29.6 & 54 & 60 & gal & 52 & 15.4 & 7.18 & 1022 & 7.60 & 155 & 0.07 & - & MRMW1S3X-W-37548 \\
\hline MW1S & $11-51$ & 05/08/16 & 15.50 & 54 & 6.5 & L & 34.11 & 14.8 & 6.93 & 999 & 8.02 & 137 & 0.00 & - & MRMW1S3X-W-39163 \\
\hline
\end{tabular}


TABLE 3.3 (Cont.)

\begin{tabular}{|c|c|c|c|c|c|c|c|c|c|c|c|c|c|c|c|}
\hline Location & $\begin{array}{l}\text { Screen } \\
\text { Interval } \\
\text { (ft BGL) }\end{array}$ & $\begin{array}{l}\text { Sample } \\
\text { Date }\end{array}$ & $\begin{array}{l}\text { Depth to } \\
\text { Water } \\
\text { (ft TOC) }\end{array}$ & $\begin{array}{l}\text { Depth of } \\
\text { Well } \\
\text { (ft TOC) }\end{array}$ & $\begin{array}{l}\text { Volume } \\
\text { Purged }\end{array}$ & $\begin{array}{l}\text { Purge } \\
\text { Units }\end{array}$ & $\begin{array}{l}\text { Pump } \\
\text { Intake } \\
\text { Position } \\
\text { (ft BGL) }\end{array}$ & $\begin{array}{c}\text { Temperature } \\
\left({ }^{\circ} \mathrm{C}\right)\end{array}$ & $\mathrm{pH}$ & $\begin{array}{c}\text { Conductivity } \\
(\mu \mathrm{S} / \mathrm{cm})\end{array}$ & $\begin{array}{c}\text { Dissolved } \\
\text { Oxygen } \\
\text { (mg/L) }\end{array}$ & $\begin{array}{l}\text { ORP } \\
(\mathrm{mV})\end{array}$ & $\begin{array}{l}\text { Iron II } \\
\text { (mg/L) }\end{array}$ & $\begin{array}{l}\text { Carbon } \\
\text { Dioxide } \\
\text { (mg/L) }\end{array}$ & Sample \\
\hline MW1S & $11-51$ & 05/08/16 & 15.50 & 54 & 6.5 & L & 40.13 & 15.2 & 6.94 & 1004 & 7.94 & 139 & 0.03 & - & MRMW1S3X-W-39164 \\
\hline MW1S & $11-51$ & $05 / 08 / 16$ & 15.50 & 54 & 6.5 & $\bar{L}$ & 46.14 & 14.7 & 6.93 & 1006 & 8.07 & 142 & 0.05 & - & MRMW1S3X-W-39165 \\
\hline MW1S & $11-51$ & $05 / 08 / 16$ & 15.50 & 54 & 80 & gal & 52 & 14.3 & 7.04 & 913 & 7.49 & 141 & 0.08 & - & MRMW1S3X-W-39166 \\
\hline MW1SU & $11-51$ & $06 / 01 / 17$ & 17.99 & 54 & 6.5 & $\mathrm{~L}$ & 24.45 & 17.7 & 6.93 & 1170 & 6.94 & 195 & 0.13 & - & MRMW1SU-W-39786 \\
\hline MW1SM & $11-51$ & $06 / 01 / 17$ & 18.09 & 54 & 6 & L & 33.3 & 16.4 & 6.94 & 1173 & 6.93 & 196 & 0.04 & - & MRMW1SM-W-39787 \\
\hline MW1SL & $11-51$ & $06 / 01 / 17$ & 18.09 & 54 & 6 & L & 42.15 & 15.3 & 6.94 & 1173 & 7.07 & 200 & 0.11 & - & MRMW1SL-W-39788 \\
\hline MW1S3 & $11-51$ & $06 / 01 / 17$ & 17.55 & 54 & 72 & gal & 52 & 14.3 & 6.98 & 877 & 6.08 & 154 & 0.06 & - & MRMW1S3-W-39789 \\
\hline MW1D & $63-88$ & $10 / 22 / 03$ & 28.39 & 88.5 & 92 & gal & _- & 14.9 & 6.87 & 2620 & _- & 25 & _- & _- & MRMW1D-W-16421 \\
\hline MW1D & $63-88$ & $06 / 02 / 04$ & 26.82 & 88.6 & 140 & gal & - & 13.9 & 6.87 & 2460 & - & - & - & - & MRMW1D-W-16458 \\
\hline MW1D & $63-88$ & 09/13/05 & 23.72 & 88.6 & 200 & gal & - & 15.5 & 6.56 & 2470 & - & - & - & - & MRMW1D-W-16518 \\
\hline MW1D & $63-88$ & 03/19/06 & 26.85 & 88.6 & 112 & gal & - & 12.9 & 6.95 & 2460 & 5.11 & 230 & 0.00 & - & MRMW1D-W-19986 \\
\hline MW1D & $63-88$ & 09/20/06 & 25.52 & 88.8 & 125 & gal & _- & 12.5 & 6.93 & 2690 & - & - & - & - & MRMW1D-W-16532 \\
\hline MW1D & $63-88$ & $03 / 21 / 07$ & 25.79 & 88.8 & 125 & gal & - & 15.3 & 6.39 & 2540 & 0.08 & 12 & 0.39 & 40 & MRMW1D-W-16487 \\
\hline MW1D & $63-88$ & $10 / 01 / 07$ & 22.75 & 89.38 & 130 & gal & - & 16.3 & 6.60 & 2230 & 6.79 & 5 & 0.44 & 45 & MRMW1D-W-16596 \\
\hline MW1D & $63-88$ & $04 / 14 / 08$ & 29.51 & 89 & 6 & $\mathrm{~L}$ & - & 14.6 & 6.99 & 2637 & 0.50 & 32 & 0.73 & - & MRMW1D-W-23231 \\
\hline MW1D & $63-88$ & $10 / 20 / 08$ & 30.4 & 89 & 7 & L & 75.5 & 13.4 & 6.83 & 2556 & 0.24 & 21 & 0.37 & - & MRMW1D-W-27621 \\
\hline MW1D & $63-88$ & $04 / 24 / 09$ & 31 & 89 & 7 & L & 75.5 & 16.7 & 6.92 & 2419 & 0.37 & 22 & 0.28 & - & MRMW1D-W-27653 \\
\hline MW1D & $63-88$ & 09/03/09 & 27.05 & 89 & 6.5 & $\mathrm{~L}$ & 75.5 & 16.9 & 7.00 & 2200 & 0.59 & 16 & 0.27 & - & MRMW1D-W-29943 \\
\hline MW1D & $63-88$ & $04 / 06 / 10$ & 24.7 & 89 & 8.5 & L & 75.5 & 17.8 & 6.39 & 2274 & 0.15 & 33 & 0.01 & - & MRMW1D-W-29983 \\
\hline MW1D & $63-88$ & $09 / 22 / 10$ & 27.92 & 89 & 9 & L & 75.5 & 17.6 & 7.01 & 2492 & 0.31 & 61 & 0.04 & - & MRMW1D-W-30011 \\
\hline MW1D & $63-88$ & $04 / 20 / 11$ & 30.15 & 89 & 5.5 & L & 75.5 & 13.5 & 6.98 & 1951 & 0.33 & -2 & 0.20 & _- & MRMW1D-W-30039 \\
\hline MW1D & $63-88$ & $10 / 04 / 11$ & 29.06 & 89 & 6.5 & L & 75.5 & 14.9 & 7.66 & 2011 & 0.17 & 22 & 0.09 & - & MRMW1D-W-30068 \\
\hline MW1D & $63-88$ & $03 / 28 / 12$ & 30.50 & 89 & 5.8 & L & 75.5 & 16.0 & 7.01 & 2161 & 0.30 & -155 & 0.00 & - & MRMW1D-W-30103 \\
\hline MW1D & $63-88$ & $05 / 07 / 13$ & 28.98 & 89 & 6 & L & 75.5 & 17.6 & 7.12 & 2132 & 0.27 & 105 & 0.13 & - & MRMW1D-W-30140 \\
\hline MW1D & $63-88$ & 06/06/14 & 33.23 & 89 & 7 & L & 75.5 & 17.7 & 6.94 & 2240 & 0.23 & 64 & 0.10 & - & MRMW1D-W-31945 \\
\hline MW1D & $63-88$ & 05/06/15 & 33.35 & 89 & 6 & $\mathrm{~L}$ & 75.5 & 17.3 & 6.88 & 2458 & 0.23 & 101 & 0.32 & - & MRMW1D-W-37549 \\
\hline MW1D & $63-88$ & $05 / 07 / 16$ & 29.72 & 89 & 5.25 & L & 75.5 & 14.2 & 6.88 & 2670 & 0.27 & 60 & 0.27 & - & MRMW1D-W-39167 \\
\hline MW1D & $63-88$ & $06 / 02 / 17$ & 29.19 & 89 & 6 & L & 75.5 & 16.2 & 6.91 & 2543 & 0.31 & 55 & 0.15 & _- & MRMW1D-W-39790 \\
\hline MW2S & $13-53$ & $10 / 22 / 03$ & 42.21 & 53.35 & $\mathrm{PDS}^{\mathrm{c}}$ & _- & _- & 16.2 & 6.86 & 875 & _- & 20 & _- & _- & MRMW02-W-16419 \\
\hline MW2S & $13-53$ & $06 / 02 / 04$ & 37.44 & 53.3 & 31 & gal & - & 16.9 & 7.07 & 861 & _- & - & - & - & MRMW2S-W-16459 \\
\hline MW2S & $13-53$ & $09 / 14 / 05$ & 33.68 & 53.33 & 38 & gal & - & 15.2 & 6.94 & 801 & 7.85 & 142 & - & 65 & MRMW2S-W-19264 \\
\hline MW2S & $13-53$ & $03 / 21 / 06$ & 40.87 & 53.32 & 27 & gal & _- & 13.0 & 7.07 & 863 & 9.40 & 262 & 0.14 & 25 & MRMW2S-W-19992 \\
\hline MW2S & $13-53$ & 09/18/06 & 36.53 & 53.3 & 28 & gal & - & 13.6 & 6.99 & 844 & 6.81 & 69 & 0.00 & 80 & MRMW2S-W-22488 \\
\hline MW2S & $13-53$ & $03 / 22 / 07$ & 35.77 & 53.3 & 35 & gal & - & 15.2 & 6.40 & 790 & 5.82 & 69 & 0.00 & 30 & MRMW2S-W-16559 \\
\hline MW2S & $13-53$ & $10 / 03 / 07$ & 31.17 & 53.4 & 44 & gal & - & 16.8 & 6.97 & 703 & 6.70 & 269 & 0.01 & 30 & MRMW2S-W-16587 \\
\hline MW2S & $13-53$ & $04 / 15 / 08$ & 23.55 & 53.4 & 2.22 & $\mathrm{~L}$ & - & 11.7 & 7.23 & 742 & 3.22 & 75 & 0.00 & - & MRMW2S-W-23232 \\
\hline MW2S & $13-53$ & $10 / 21 / 08$ & 33.7 & 53.5 & 5 & L & 33 & 13.4 & 7.08 & 745 & 8.55 & 104 & 0.00 & - & MRMW2S-W-27622 \\
\hline MW2S & $13-53$ & $10 / 21 / 08$ & - & - & TWV & - & - & - & - & - & - & - & - & - & MRMW2S-W-27652 \\
\hline MW2S & $13-53$ & $04 / 23 / 09$ & 33.2 & 53.5 & 6.5 & $\mathrm{~L}$ & 43.35 & 15.2 & 6.97 & 755 & 9.19 & 26 & 0.00 & _- & MRMW2S-W-27654 \\
\hline MW2S & $13-53$ & 09/03/09 & 29.4 & 53.5 & 5.4 & L & 41.2 & 15.1 & 7.15 & 690 & 8.80 & 32 & 0.01 & - & MRMW2S-W-29944 \\
\hline MW2S & $13-53$ & $04 / 06 / 10$ & 18.57 & 52.42 & 6.5 & L & 36 & 17.1 & 6.95 & 681 & 6.85 & 37 & 0.12 & - & MRMW2S-W-29984 \\
\hline MW2S & $13-53$ & $09 / 22 / 10$ & 31.25 & 53 & 7 & L & 33 & 16.1 & 7.03 & 723 & 7.16 & 142 & 0.00 & - & MRMW2S-W-30012 \\
\hline MW2S & $13-53$ & 04/20/11 & 36.00 & 53.5 & 8 & L & 44.5 & 13.4 & 6.91 & 615 & 7.07 & 103 & 0.05 & - & MRMW2S-W-30040 \\
\hline MW2S & $13-53$ & $10 / 04 / 11$ & 32.73 & 53.5 & 7.5 & L & 42.6 & 15.3 & 7.71 & 592 & 6.89 & 120 & 0.01 & - & MRMW2S-W-30069 \\
\hline MW2S & $13-53$ & $03 / 28 / 12$ & 31.45 & 53.5 & 7 & L & 42.48 & 15.8 & 7.06 & 629 & 7.04 & -59 & 0.00 & - & MRMW2S-W-30104 \\
\hline MW2S & $13-53$ & $05 / 07 / 13$ & 32.86 & 53.5 & 5 & L & 42.93 & 14.9 & 7.09 & 633 & 7.64 & 37 & 0.00 & - & MRMW2S-W-30141 \\
\hline
\end{tabular}


TABLE 3.3 (Cont.)

\begin{tabular}{|c|c|c|c|c|c|c|c|c|c|c|c|c|c|c|c|}
\hline Location & $\begin{array}{l}\text { Screen } \\
\text { Interval } \\
\text { (ft BGL) }\end{array}$ & $\begin{array}{l}\text { Sample } \\
\text { Date }\end{array}$ & $\begin{array}{l}\text { Depth to } \\
\text { Water } \\
\text { (ft TOC) }\end{array}$ & $\begin{array}{l}\text { Depth of } \\
\text { Well } \\
\text { (ft TOC) }\end{array}$ & $\begin{array}{l}\text { Volume } \\
\text { Purged }\end{array}$ & $\begin{array}{l}\text { Purge } \\
\text { Units }\end{array}$ & $\begin{array}{l}\text { Pump } \\
\text { Intake } \\
\text { Position } \\
\text { (ft BGL) }\end{array}$ & $\begin{array}{c}\text { Temperature } \\
\left({ }^{\circ} \mathrm{C}\right)\end{array}$ & $\mathrm{pH}$ & $\begin{array}{c}\text { Conductivity } \\
(\mu \mathrm{S} / \mathrm{cm})\end{array}$ & $\begin{array}{l}\text { Dissolved } \\
\text { Oxygen } \\
\text { (mg/L) }\end{array}$ & $\begin{array}{l}\text { ORP } \\
(\mathrm{mV})\end{array}$ & $\begin{array}{l}\text { Iron II } \\
\text { (mg/L) }\end{array}$ & $\begin{array}{c}\text { Carbon } \\
\text { Dioxide } \\
\text { (mg/L) }\end{array}$ & Sample \\
\hline MW2S & $13-53$ & 06/06/14 & 37.03 & 53.5 & 8 & L & 45.42 & 15.3 & 7.06 & 777 & 7.55 & 134 & 0.00 & - & MRMW2S-W-31946 \\
\hline MW2S & $13-53$ & 05/06/15 & 38.80 & 53.5 & 7 & $\mathrm{~L}$ & 46.05 & 16.5 & 7.02 & 827 & 6.39 & 193 & 0.05 & - & MRMW2S-W-37550 \\
\hline MW2S & $13-53$ & 05/07/16 & 18.16 & 53.5 & 7 & $\mathrm{~L}$ & 46.05 & 17.1 & 6.94 & 756 & 7.40 & 161 & 0.10 & - & MRMW2S-W-39168 \\
\hline MW2S & $13-53$ & 06/01/17 & 24.06 & 53.5 & 5.5 & $\mathrm{~L}$ & 38.77 & 15.1 & 7.05 & 731 & 7.46 & 210 & 0.20 & - & MRMW2S-W-39791 \\
\hline MW3S & $18-48$ & $10 / 23 / 03$ & 36.47 & 47.79 & 73 & gal & - & 16.8 & 7.23 & 655 & - & 6 & - & - & MRMW03-W-16423 \\
\hline MW3S & $18-48$ & $06 / 02 / 04$ & 30.67 & 47.5 & 34 & gal & - & 14.2 & 7.23 & 664 & - & - & - & - & MRMW3S-W-16462 \\
\hline MW3S & $18-48$ & 09/13/05 & 25.6 & 47.6 & 50 & gal & - & 14.6 & 7.13 & 663 & 8.82 & 223 & 0.00 & 100 & MRMW3S-W-19261 \\
\hline MW3S & $18-48$ & 03/23/06 & 35.62 & 47.74 & 28 & gal & - & 8.9 & 7.16 & 662 & 6.74 & 269 & 0.08 & 25 & MRMW3S-W-19994 \\
\hline MW3S & $18-48$ & 09/20/06 & 29.44 & 47.75 & 22 & gal & - & 12.9 & 7.15 & 669 & 7.64 & 105 & 0.00 & - & MRMW3S-W-22496 \\
\hline MW3S & $18-48$ & 03/22/07 & 26.19 & 47.75 & 45 & gal & - & 15.0 & 6.44 & 578 & 5.90 & 261 & 0.17 & 30 & MRMW3S-W-16563 \\
\hline MW3S & $18-48$ & $10 / 03 / 07$ & 22.7 & 47.9 & 50 & gal & - & 15.3 & 6.97 & 594 & 0.38 & 282 & 0.00 & 20 & MRMW3S-W-16585 \\
\hline MW3S & $18-48$ & 04/14/08 & 16.95 & 47.8 & 3.31 & $\mathrm{~L}$ & - & 13.7 & 7.17 & 693 & 3.52 & 165 & 0.00 & - & MRMW3S-W-23233 \\
\hline MW3S & $18-48$ & $04 / 22 / 08$ & 15.8 & 47.8 & 6.5 & L & - & 16.0 & 6.99 & 685 & 6.71 & 155 & - & - & MRMW3S-W-23260 \\
\hline MW3S & $18-48$ & 05/01/08 & - & - & 2.62 & L & 26 & 13.2 & 7.17 & 675 & 3.83 & 161 & - & - & MRMW3S-26-W-23269 \\
\hline MW3S & $18-48$ & 05/01/08 & - & - & 2.75 & $\mathrm{~L}$ & 38 & 12.7 & 7.12 & 671 & 4.21 & 193 & - & - & MRMW3S-38-W-23270 \\
\hline MW3S & $18-48$ & 05/01/08 & - & - & 3.17 & L & 45 & 12.6 & 7.03 & 675 & 4.57 & 205 & - & - & MRMW3S-45-W-23271 \\
\hline MW3S & $18-48$ & $10 / 21 / 08$ & 27 & 47.8 & 4.2 & L & 33 & 12.6 & 7.17 & 673 & 6.42 & 115 & 0.00 & - & MRMW3S-W-27623 \\
\hline MW3S & $18-48$ & $10 / 21 / 08$ & - & - & TWV & - & - & 14.3 & 7.11 & 522 & - & - & 0.00 & - & MRMW3S-W-27650 \\
\hline MW3S & $18-48$ & $04 / 23 / 09$ & 26.7 & 47.8 & 5 & L & 37.3 & 17.0 & 7.06 & 662 & 9.40 & -63 & 0.01 & - & MRMW3S-W-27655 \\
\hline MW3S & $18-48$ & 09/03/09 & 22.4 & 47.8 & 5.5 & $\mathrm{~L}$ & 35.2 & 16.3 & 7.28 & 640 & 7.69 & 12 & 0.01 & - & MRMW3S-W-29945 \\
\hline MW3S & $18-48$ & 09/04/09 & 22.55 & 47.8 & 190 & L & 46 & 14.2 & 6.57 & 659 & 9.09 & 95 & - & - & MRMW3S-W-29972 \\
\hline MW3S & $18-48$ & $04 / 06 / 10$ & 12.14 & 47.8 & 6 & L & 33 & 16.0 & 6.99 & 611 & 8.20 & 8 & 0.00 & - & MRMW3S-W-29985 \\
\hline MW3S & $18-48$ & 09/23/10 & 23.73 & 47.8 & 7 & $\mathrm{~L}$ & 33 & 15.8 & 7.22 & 674 & 11.00 & 150 & 0.05 & - & MRMW3S-W-30013 \\
\hline MW3S & $18-48$ & $04 / 20 / 11$ & 32.06 & 47.8 & 6.2 & L & 40 & 13.3 & 7.11 & 514 & 6.64 & 61 & 0.02 & - & MRMW3S-W-30041 \\
\hline MW3S & $18-48$ & $10 / 04 / 11$ & 25.95 & 47.8 & 7 & $\mathrm{~L}$ & 37 & 15.8 & 7.78 & 563 & 5.99 & 115 & 0.00 & - & MRMW3S-W-30070 \\
\hline MW3S & $18-48$ & $03 / 28 / 12$ & 23.95 & 47.8 & 6.5 & L & 35.88 & 15.2 & 7.11 & 592 & 7.33 & -56 & 0.00 & - & MRMW3S-W-30105 \\
\hline MW3S & $18-48$ & $05 / 07 / 13$ & 25.65 & 47.8 & 7 & L & 36.83 & 14.8 & 7.16 & 547 & 8.68 & 38 & 0.00 & - & MRMW3S-W-30142 \\
\hline MW3S & $18-48$ & 06/06/14 & 34.46 & 47.80 & 7.25 & $\mathrm{~L}$ & 41.39 & 16.3 & 7.11 & 644 & 8.80 & 137 & 0.00 & - & MRMW3S-W-31947 \\
\hline MW3S & $18-48$ & 04/30/15 & 33.95 & 47.80 & 7 & L & 42.1 & 15.3 & 7.25 & 709 & 6.61 & 194 & 0.05 & - & MRMW3S-W-37551 \\
\hline MW3S & $18-48$ & 05/07/16 & 12.60 & 47.80 & 7 & $\mathrm{~L}$ & 42.1 & 18.6 & 6.99 & 673 & 8.61 & 151 & 0.00 & - & MRMW3S-W-39169 \\
\hline MW3S & $18-48$ & 06/01/17 & 17.79 & 47.8 & 6 & L & 33.04 & 15.6 & 7.08 & 662 & 7.35 & 214 & 0.04 & - & MRMW3S-W-39792 \\
\hline MW4S & $17-47$ & $10 / 21 / 03$ & 46.4 & 47.8 & PDS & - & - & - & 7.17 & 758 & - & - & - & - & MRMW04-W-16418 \\
\hline MW4S & $17-47$ & 06/04/04 & 43.21 & 47.8 & 10 & gal & - & 15.4 & 6.93 & 769 & - & - & - & - & MRMW4S-W-16470 \\
\hline MW4S & $17-47$ & $09 / 14 / 05$ & 36.21 & 47.81 & 8 & gal & - & 15.4 & 7.30 & 751 & 8.00 & 174 & 0.00 & 50 & MRMW4S-W-19262 \\
\hline MW4S & $17-47$ & 03/21/06 & 44.55 & 47.72 & 6 & gal & - & 6.7 & 7.25 & 729 & 10.90 & 154 & 0.00 & 25 & MRMW4S-W-19993 \\
\hline MW4S & $17-47$ & 09/18/06 & 41.56 & 47.75 & 5.25 & gal & - & 13.1 & 7.25 & 728 & 8.05 & 41 & 0.00 & 50 & MRMW4S-W-22487 \\
\hline MW4S & $17-47$ & 03/22/07 & 38.74 & 47.75 & 6 & gal & - & 14.2 & 6.53 & 765 & 5.91 & 78 & 0.10 & 25 & MRMW4S-W-16562 \\
\hline MW4S & $17-47$ & $10 / 03 / 07$ & 31.11 & 47.73 & 30 & gal & - & 16.4 & 6.95 & 715 & 7.40 & 281 & 0.10 & 30 & MRMW4S-W-16586 \\
\hline MW4S & $17-47$ & $01 / 11 / 08$ & - & - & - & - & - & 11.3 & 7.56 & 757 & - & - & - & - & MOMW4S-W-011108 \\
\hline MW4S & $17-47$ & $04 / 14 / 08$ & 26.32 & 47.85 & 2.48 & $\mathrm{~L}$ & - & 13.1 & 7.28 & 783 & 3.80 & 213 & 0.00 & - & MRMW4S-W-23234 \\
\hline MW4S & $17-47$ & $10 / 20 / 08$ & 36.7 & 47.8 & 8 & gal & - & 14.5 & 7.16 & 756 & 8.71 & 104 & 0.00 & - & MRMW4S-W-27624 \\
\hline MW4S & $17-47$ & $04 / 23 / 09$ & 41.5 & 47.8 & 5 & $\mathrm{~L}$ & 44.65 & 15.8 & 7.16 & 717 & 9.45 & 22 & 0.05 & - & MRMW4S-W-27656 \\
\hline MW4S & $17-47$ & $09 / 04 / 09$ & 31.6 & 47.8 & 6 & L & 39.3 & 15.0 & 7.13 & 731 & 8.23 & 80 & 0.04 & - & MRMW4S-W-29946 \\
\hline MW4S & $17-47$ & $04 / 06 / 10$ & 21.8 & 47.85 & 5.5 & L & 34.83 & 16.5 & 7.07 & 629 & 8.07 & -1 & 0.00 & - & MRMW4S-W-29986 \\
\hline MW4S & $17-47$ & $09 / 22 / 10$ & 33.15 & 47.8 & 6 & L & 32 & 16.3 & 7.07 & 732 & 7.28 & 90 & 0.00 & - & MRMW4S-W-30014 \\
\hline MW4S & $17-47$ & 04/21/11 & 42.39 & 47.8 & 5 & L & 44.7 & 12.6 & 7.15 & 595 & 7.25 & 138 & 0.00 & - & MRMW4S-W-30042 \\
\hline
\end{tabular}


TABLE 3.3 (Cont.)

\begin{tabular}{|c|c|c|c|c|c|c|c|c|c|c|c|c|c|c|c|}
\hline Location & $\begin{array}{l}\text { Screen } \\
\text { Interval } \\
\text { (ft BGL) }\end{array}$ & $\begin{array}{l}\text { Sample } \\
\text { Date }\end{array}$ & $\begin{array}{l}\text { Depth to } \\
\text { Water } \\
\text { (ft TOC) }\end{array}$ & $\begin{array}{l}\text { Depth of } \\
\text { Well } \\
\text { (ft TOC) }\end{array}$ & $\begin{array}{l}\text { Volume } \\
\text { Purged }\end{array}$ & $\begin{array}{l}\text { Purge } \\
\text { Units }\end{array}$ & $\begin{array}{l}\text { Pump } \\
\text { Intake } \\
\text { Position } \\
\text { (ft BGL) }\end{array}$ & $\begin{array}{c}\text { Temperature } \\
\left({ }^{\circ} \mathrm{C}\right)\end{array}$ & $\mathrm{pH}$ & $\begin{array}{c}\text { Conductivity } \\
(\mu \mathrm{S} / \mathrm{cm})\end{array}$ & $\begin{array}{l}\text { Dissolved } \\
\text { Oxygen } \\
\text { (mg/L) }\end{array}$ & $\begin{array}{l}\text { ORP } \\
(\mathrm{mV})\end{array}$ & $\begin{array}{l}\text { Iron II } \\
(\mathrm{mg} / \mathrm{L})\end{array}$ & $\begin{array}{l}\text { Carbon } \\
\text { Dioxide } \\
\text { (mg/L) }\end{array}$ & Sample \\
\hline MW4S & $17-47$ & $10 / 04 / 11$ & 35.6 & 47.8 & 6 & L & 40.9 & 16.0 & 7.95 & 606 & 7.62 & 115 & 0.00 & - & MRMW4S-W-30071 \\
\hline MW4S & $17-47$ & $03 / 28 / 12$ & 35.10 & 47.8 & 7.2 & L & 41.45 & 14.5 & 6.97 & 612 & 7.78 & -37 & 0.01 & - & MRMW4S-W-30106 \\
\hline MW4S & $17-47$ & $05 / 07 / 13$ & 44.2 & 47.8 & 9 & L & 45.6 & 15.3 & 7.46 & 582 & 8.45 & 39 & 0.03 & - & MRMW4S-W-30143 \\
\hline MW4S & $17-47$ & $06 / 06 / 14$ & $N S^{d}$ & - & - & - & - & - & - & - & - & - & - & - & - \\
\hline MW4S & $17-47$ & 04/30/15 & $N S^{d}$ & - & - & - & - & - & - & - & - & - & - & - & - \\
\hline MW4S & $17-47$ & 05/08/16 & 26.28 & 54.6 & NR & L & 40.88 & 14.0 & 7.10 & 774 & 9.11 & 116 & 0.00 & - & MRMW4S-W-39170 \\
\hline MW4S & $17-47$ & 06/02/17 & 27.04 & 47.8 & 6.5 & $\mathrm{~L}$ & 37.14 & 14.8 & 7.15 & 742 & 5.63 & 160 & 0.05 & - & MRMW4S-W-39793 \\
\hline MW5S & $15-55$ & $10 / 22 / 03$ & 31.4 & 55.72 & 48 & gal & - & 15.3 & 7.10 & 816 & - & 6 & - & - & MRMW05-W-16420 \\
\hline MW5S & $15-55$ & $06 / 02 / 04$ & 26.33 & 55.65 & $>57$ & gal & - & 14.3 & 7.21 & 817 & - & - & - & - & MRMW5S-W-16460 \\
\hline MW5S & $15-55$ & 09/13/05 & 22.66 & 54.2 & 75 & gal & - & 16.0 & 7.04 & 763 & 13.90 & 228 & 0.00 & 60 & MRMW5S-W-19260 \\
\hline MW5S & $15-55$ & $03 / 22 / 06$ & 28.64 & 54.51 & 50 & gal & - & 13.9 & 7.25 & 781 & 4.52 & 234 & 0.06 & 35 & MRMW5S-W-19996 \\
\hline MW5S & $15-55$ & 09/20/06 & 25.43 & 54.63 & 52 & gal & - & 13.9 & 7.19 & 787 & 5.82 & 73 & 0.00 & 35 & MRMW5S-W-22493 \\
\hline MW5S & $15-55$ & 03/22/07 & 25.14 & 54.56 & 58 & gal & - & 15.5 & 6.50 & 436 & 3.98 & 159 & 0.08 & 30 & MRMW5S-W-16569 \\
\hline MW5S & $15-55$ & $10 / 03 / 07$ & 19.55 & 54.65 & 68 & gal & - & 16.5 & 7.18 & 850 & 1.87 & 268 & 0.04 & 25 & MRMW5S-W-16588 \\
\hline MW5S & $15-55$ & $04 / 14 / 08$ & 11.2 & 54.6 & 6 & $\mathrm{~L}$ & - & 14.1 & 6.90 & 1008 & 3.73 & 143 & 0.02 & - & MRMW5S-W-23235 \\
\hline MW5S & $15-55$ & $04 / 23 / 08$ & 11.3 & 54.6 & 6.5 & L & - & 14.9 & 6.88 & 1009 & 3.27 & 184 & - & - & MRMW5S-W-23266 \\
\hline MW5S & $15-55$ & 05/01/08 & - & - & 3.7 & $\mathrm{~L}$ & 20 & 15.2 & 6.92 & 1014 & 2.99 & 126 & - & - & MRMW5S-20-W-23272 \\
\hline MW5S & $15-55$ & 05/01/08 & - & - & 3.4 & $\mathrm{~L}$ & 28 & 15.1 & 6.90 & 997 & 2.38 & 124 & - & - & MRMW5S-28-W-23273 \\
\hline MW5S & $15-55$ & $05 / 01 / 08$ & - & - & 4 & L & 52 & 15.1 & 6.89 & 989 & 2.06 & 128 & - & - & MRMW5S-52-W-23274 \\
\hline MW5S & $15-55$ & $10 / 21 / 08$ & 22.5 & 54.6 & 7 & L & 35 & 13.2 & 7.04 & 818 & 5.54 & 180 & 0.00 & - & MRMW5S-W-27625 \\
\hline MW5S & $15-55$ & $04 / 24 / 09$ & 22.1 & 54.6 & 5.5 & $\bar{L}$ & 38.35 & 15.2 & 6.98 & 817 & 6.68 & 77 & 0.00 & - & MRMW5S-W-27657 \\
\hline MW5S & $15-55$ & 09/03/09 & 17.6 & 54.6 & 5.5 & L & 36.3 & 16.5 & 7.10 & 873 & 3.07 & 23 & 0.02 & - & MRMW5S-W-29947 \\
\hline MW5S & $15-55$ & $04 / 07 / 10$ & 8.32 & 54.5 & 5.5 & $\mathrm{~L}$ & 35 & 12.6 & 6.76 & 844 & 4.46 & 149 & 0.00 & - & MRMW5S-W-29987 \\
\hline MW5S & $15-55$ & $09 / 22 / 10$ & 19.25 & 55 & 6.5 & L & 35 & 16.4 & 6.81 & 891 & 1.69 & 112 & 0.00 & - & MRMW5S-W-30015 \\
\hline MW5S & $15-55$ & $04 / 20 / 11$ & 24.43 & 54.6 & 7 & L & 36 & 14.6 & 7.17 & 617 & 5.34 & 89.4 & 0.00 & - & MRMW5S-W-30043 \\
\hline MW5S & $15-55$ & $10 / 04 / 11$ & 21.10 & 54.6 & 6.5 & $\mathrm{~L}$ & 38.25 & 16.7 & 7.72 & 740 & 4.37 & 118.4 & 0.03 & - & MRMW5S-W-30072 \\
\hline MW5S & $15-55$ & 03/28/12 & 20.60 & 54.6 & 7 & L & 37.6 & 16.9 & 7.11 & 724 & 5.29 & -65.8 & 0.00 & - & MRMW5S-W-30107 \\
\hline MW5S & $15-55$ & $05 / 07 / 13$ & 21.9 & 54.6 & 5 & L & 38.45 & 14.2 & 7.27 & 694 & 5.24 & 51 & 0.05 & - & MRMW5S-W-30144 \\
\hline MW5S & $15-55$ & $06 / 06 / 14$ & 25.04 & 54.6 & 8 & L & 40.19 & 16.4 & 7.16 & 730 & 6.41 & 133 & 0.02 & - & MRMW5S-W-31949 \\
\hline MW5S & $15-55$ & $05 / 06 / 15$ & 26.5 & 54.6 & 5 & L & 40.88 & 16.3 & 6.99 & 860 & 4.52 & 207 & 0.04 & - & MRMW5S-W-37553 \\
\hline MW5S & $15-55$ & 05/07/16 & 8.27 & 54.6 & 6 & $\mathrm{~L}$ & 35 & 14.7 & 6.97 & 881 & 6.18 & 75 & 0.00 & - & MRMW5S-W-39171 \\
\hline MW5S & $15-55$ & 06/01/17 & 12.02 & 54.6 & 6 & $\mathrm{~L}$ & 35 & 15.9 & 6.85 & 900 & 4.68 & 210 & 0.13 & - & MRMW5S-W-39794 \\
\hline MW6S & $10-25$ & 06/03/04 & 3.34 & 26.9 & 45 & gal & - & 15.1 & 6.89 & 2410 & - & - & - & - & MRMW6S-W-16465 \\
\hline MW6S & $10-25$ & 09/14/05 & 4.7 & 26.9 & 43 & gal & - & 14.1 & 7.06 & 2350 & 0.01 & 54 & 0.00 & 60 & MRMW6S-W-19263 \\
\hline MW6S & $10-25$ & 03/20/06 & 5.35 & 26.91 & 43 & gal & - & 9.8 & 6.91 & 2360 & 1.37 & 89 & 0.38 & 60 & MRMW6S-W-19990 \\
\hline MW6S & $10-25$ & 09/18/06 & 5.48 & 26.92 & 27 & gal & - & 12.5 & 6.96 & 2410 & 0.08 & -29 & 0.35 & 85 & MRMW6S-W-22486 \\
\hline MW6S & $10-25$ & $03 / 21 / 07$ & 5.42 & 26.92 & 30 & gal & - & 18.0 & 6.34 & 2450 & 0.12 & 75 & 0.78 & 40 & MRMW6S-W-16486 \\
\hline MW6S & $10-25$ & $10 / 02 / 07$ & 5 & 26.9 & 31 & gal & - & 17.1 & 7.33 & 2280 & 0.20 & 61 & 0.19 & 35 & MRMW6S-W-16583 \\
\hline MW6S & $10-25$ & 04/15/08 & 5.15 & 26.9 & 2.5 & L & - & 8.7 & 6.99 & 2485 & 0.31 & -76 & 0.41 & - & MRMW6S-W-23236 \\
\hline MW6S & $10-25$ & $10 / 20 / 08$ & 5.7 & 26.9 & 5 & L & 17.5 & 14.3 & 6.84 & 2380 & 0.36 & 18 & 0.28 & - & MRMW6S-W-27626 \\
\hline MW6S & $10-25$ & $04 / 24 / 09$ & 6.2 & 26.9 & 12 & L & 17.5 & 15.2 & 6.93 & 2270 & 0.19 & -39 & 0.63 & - & MRMW6S-W-27658 \\
\hline MW6S & $10-25$ & $09 / 04 / 09$ & 5.85 & 26.9 & 5.4 & L & 17.5 & 13.8 & 6.88 & 2302 & 0.64 & 79 & 0.32 & - & MRMW6S-W-29948 \\
\hline MW6S & $10-25$ & $04 / 06 / 10$ & 6.15 & 26.9 & 8 & L & 17.5 & 15.2 & 6.87 & 2141 & 0.05 & -19 & 0.21 & - & MRMW6S-W-29988 \\
\hline MW6S & $10-25$ & 09/22/10 & 5.53 & 26.9 & 8.75 & L & 17.5 & 15.6 & 6.92 & 2354 & 0.42 & 47 & 0.24 & - & MRMW6S-W-30016 \\
\hline MW6S & $10-25$ & 04/20/11 & 6.52 & 24.88 & 8 & L & 17.5 & 13.0 & 6.73 & 1867 & 0.18 & 28 & 0.09 & - & MRMW6S-W-30044 \\
\hline MW6S & $10-25$ & $10 / 04 / 11$ & 6.49 & 26.9 & 6 & L & 17.5 & 16.6 & 7.11 & 2020 & 0.14 & 14 & 0.34 & - & MRMW6S-W-30073 \\
\hline
\end{tabular}


TABLE 3.3 (Cont.)

\begin{tabular}{|c|c|c|c|c|c|c|c|c|c|c|c|c|c|c|c|}
\hline Location & $\begin{array}{l}\text { Screen } \\
\text { Interval } \\
\text { (ft BGL) }\end{array}$ & $\begin{array}{l}\text { Sample } \\
\text { Date }\end{array}$ & $\begin{array}{l}\text { Depth to } \\
\text { Water } \\
\text { (ft TOC) }\end{array}$ & $\begin{array}{l}\text { Depth of } \\
\text { Well } \\
\text { (ft TOC) }\end{array}$ & $\begin{array}{l}\text { Volume } \\
\text { Purged }\end{array}$ & $\begin{array}{l}\text { Purge } \\
\text { Units }\end{array}$ & $\begin{array}{l}\text { Pump } \\
\text { Intake } \\
\text { Position } \\
\text { (ft BGL) }\end{array}$ & $\begin{array}{c}\text { Temperature } \\
\left({ }^{\circ} \mathrm{C}\right)\end{array}$ & $\mathrm{pH}$ & $\begin{array}{c}\text { Conductivity } \\
(\mu \mathrm{S} / \mathrm{cm})\end{array}$ & $\begin{array}{l}\text { Dissolved } \\
\text { Oxygen } \\
\text { (mg/L) }\end{array}$ & $\begin{array}{l}\text { ORP } \\
(\mathrm{mV})\end{array}$ & $\begin{array}{l}\text { Iron II } \\
(\mathrm{mg} / \mathrm{L})\end{array}$ & $\begin{array}{l}\text { Carbon } \\
\text { Dioxide } \\
\text { (mg/L) }\end{array}$ & Sample \\
\hline MW6S & $10-25$ & 03/28/12 & 6.38 & 26.9 & 7 & L & 17.5 & 14.7 & 6.62 & 1928 & 0.20 & 0 & 0.00 & - & MRMW6S-W-30108 \\
\hline MW6S & $10-25$ & $05 / 07 / 13$ & 6.3 & 26.9 & 6.5 & L & 17.5 & 12.7 & 7.23 & 1672 & 0.52 & -11 & 0.63 & - & MRMW6S-W-30145 \\
\hline MW6S & $10-25$ & $06 / 05 / 14$ & 6.54 & 26.9 & 9.25 & L & 16.01 & 14.4 & 6.90 & 2042 & 0.55 & 16 & 0.15 & - & MRMW6S-W-31950 \\
\hline MW6S & $10-25$ & $04 / 29 / 15$ & 6.6 & 26.9 & 9 & L & 17.5 & 13.6 & 6.98 & 2023 & 0.15 & 46 & 0.53 & - & MRMW6S-W-37554 \\
\hline MW6S & $10-25$ & 05/07/16 & 5.36 & 26.9 & 7.75 & L & 17.5 & 14.3 & 6.90 & 2170 & 0.20 & -25 & 0.83 & - & MRMW6S-W-39172 \\
\hline MW6S & $10-25$ & $06 / 02 / 17$ & 5.93 & 26.9 & 6.5 & L & 17.5 & 14.9 & 6.97 & 2017 & 0.19 & 5 & 0.90 & - & MRMW6S-W-39795 \\
\hline MW7S & $20-45$ & 06/03/04 & 26.68 & 46.98 & 40 & gal & - & 13.8 & 7.19 & 763 & - & - & - & - & MRMW7S-W-16466 \\
\hline MW7S & $20-45$ & 09/12/05 & 17.57 & 46.94 & 55 & gal & - & 15.0 & 7.26 & 760 & 8.35 & 240 & 0.00 & 50 & MRMW7S-W-19258 \\
\hline MW7S & $20-45$ & 03/22/06 & 22.45 & 46.98 & 48 & gal & - & 15.2 & 7.32 & 740 & 5.52 & 268 & 0.03 & 25 & MRMW7S-W-20000 \\
\hline MW7S & $20-45$ & 09/19/06 & 20.94 & 47.02 & 56 & gal & - & 13.2 & 7.15 & 764 & 7.37 & 114 & 0.00 & 25 & MRMW7S-W-22490 \\
\hline MW7S & $20-45$ & 03/20/07 & 18.01 & 47.02 & 50 & gal & - & 14.6 & 6.43 & 750 & 5.31 & 95 & 0.00 & 30 & MRMW7S-W-16481 \\
\hline MW7S & $20-45$ & $10 / 01 / 07$ & 12.4 & 47 & 70 & gal & - & 15.6 & 6.99 & 725 & 7.76 & 269 & 0.01 & 35 & MRMW7S-W-16581 \\
\hline MW7S & $20-45$ & $04 / 14 / 08$ & 7.72 & 47 & 1.82 & $\mathrm{~L}$ & - & 13.4 & 7.21 & 811 & 2.50 & 276 & 0.00 & - & MRMW7S-W-23237 \\
\hline MW7S & $20-45$ & $04 / 23 / 08$ & 7.8 & 47 & 11 & L & - & 14.0 & 7.00 & 822 & 7.41 & 191 & - & - & MRMW7S-W-23265 \\
\hline MW7S & $20-45$ & $10 / 20 / 08$ & 17.2 & 47 & 6.3 & L & 32.5 & 14.9 & 7.02 & 802 & 6.38 & 87 & 0.00 & - & MRMW7S-W-27627 \\
\hline MW7S & $20-45$ & $04 / 23 / 09$ & 16.7 & 47 & 7 & L & 32.5 & 16.1 & 7.01 & 727 & 9.48 & -53 & 0.01 & - & MRMW7S-W-27659 \\
\hline MW7S & $20-45$ & $09 / 03 / 09$ & 13.8 & 47 & 9 & $\mathrm{~L}$ & 32.5 & 17.4 & 7.58 & 814 & 9.86 & 102 & 0.02 & - & MRMW7S-W-29949 \\
\hline MW7S & $20-45$ & $04 / 06 / 10$ & 6.38 & 47 & 5.4 & L & 32.5 & 14.7 & 7.04 & 718 & 7.92 & 23 & 0.00 & - & MRMW7S-W-29989 \\
\hline MW7S & $20-45$ & $09 / 23 / 10$ & 14.15 & 45 & 6 & $\mathrm{~L}$ & 32.5 & 16.0 & 6.91 & 772 & 8.39 & 201 & 0.00 & - & MRMW7S-W-30017 \\
\hline MW7S & $20-45$ & 04/20/11 & 19.69 & 46.69 & 6 & L & 32.5 & 13.0 & 7.00 & 598 & 7.91 & 128 & 0.00 & - & MRMW7S-W-30045 \\
\hline MW7S & $20-45$ & $10 / 04 / 11$ & 7.11 & 47 & 7 & L & 32.5 & 16.2 & 7.18 & 674 & 8.17 & 205 & 0.01 & - & MRMW7S-W-30074 \\
\hline MW7S & $20-45$ & 03/28/12 & 15.35 & 47 & 8 & L & 32.5 & 16.7 & 6.69 & 686 & 8.01 & 165 & 0.04 & - & MRMW7S-W-30109 \\
\hline MW7S & $20-45$ & $05 / 07 / 13$ & 19.4 & 47 & 6.25 & L & 32.5 & 13.4 & 7.30 & 855 & 5.98 & 228 & 0.02 & - & MRMW7S-W-30146 \\
\hline MW7S & $20-45$ & $06 / 06 / 14$ & 21.65 & 47 & 7.5 & L & 33.75 & 15.2 & 7.10 & 1053 & 6.91 & 117 & 0.02 & - & MRMW7S-W-31951 \\
\hline MW7S & $20-45$ & $04 / 29 / 15$ & 23.4 & 47 & 6 & L & 34.53 & 16.6 & 7.22 & 776 & 7.40 & 179 & 0.07 & - & MRMW7S-W-37555 \\
\hline MW7S & $20-45$ & 05/06/16 & 10.03 & 47 & 7.5 & L & 32.5 & 14.7 & 7.06 & 823 & 8.55 & 174 & 0.07 & - & MRMW7S-W-39173 \\
\hline MW7S & $20-45$ & 05/31/17 & 10.02 & 47 & 6 & $\mathrm{~L}$ & 32.5 & 14.7 & 7.17 & 778 & 7.56 & 125 & 0.08 & - & MRMW7S-W-39796 \\
\hline MW8S & $10-25$ & $06 / 03 / 04$ & 3.7 & 26.75 & 45 & gal & - & 12.8 & 7.12 & 941 & - & - & - & - & MRMW8S-W-16464 \\
\hline MW8S & $10-25$ & $09 / 14 / 05$ & 4.02 & 26.82 & 57 & gal & - & 14.1 & 7.30 & 853 & 0.02 & 65 & 0.00 & 40 & MRMW8S-W-19265 \\
\hline MW8S & $10-25$ & 03/20/06 & 4.57 & 26.41 & 43 & gal & - & 12.5 & 7.04 & 954 & 0.90 & 153 & 0.05 & 30 & MRMW8S-W-19991 \\
\hline MW8S & $10-25$ & 09/19/06 & 4.76 & 26.83 & 45 & gal & - & 11.8 & 7.09 & 903 & 0.58 & 284 & 0.13 & 50 & MRMW8S-W-22492 \\
\hline MW8S & $10-25$ & 03/20/07 & 2.63 & 26.82 & 49 & gal & - & 11.0 & 6.52 & 1026 & 0.77 & 76 & 0.00 & 30 & MRMW8S-W-16483 \\
\hline MW8S & $10-25$ & $10 / 02 / 07$ & 2.2 & 26.8 & 48 & gal & - & 15.2 & 6.76 & 607 & 2.66 & 209 & 0.02 & 25 & MRMW8S-W-16584 \\
\hline MW8S & $10-25$ & 04/15/08 & 0.7 & 26.8 & 5.5 & L & - & 10.2 & 7.27 & 1067 & 1.58 & 170 & 0.00 & - & MRMW8S-W-23238 \\
\hline MW8S & $10-25$ & $10 / 20 / 08$ & 3.6 & 26.8 & 8 & L & 17.5 & 14.0 & 6.91 & 1002 & 0.93 & 69 & 0.00 & - & MRMW8S-W-27628 \\
\hline MW8S & $10-25$ & $04 / 23 / 09$ & 2.3 & 26.8 & 6 & L & 17.5 & 11.4 & 6.88 & 825 & 1.76 & -35 & 0.02 & - & MRMW8S-W-27660 \\
\hline MW8S & $10-25$ & 09/03/09 & 2.9 & 26.8 & 8.5 & L & 17.5 & 14.1 & 7.52 & 890 & 2.09 & 115 & 0.00 & - & MRMW8S-W-29950 \\
\hline MW8S & $10-25$ & $04 / 06 / 10$ & 1.1 & 26.8 & 8 & L & 17.5 & 13.7 & 7.00 & 843 & 2.60 & 212 & 0.00 & - & MRMW8S-W-29990 \\
\hline MW8S & $10-25$ & 09/22/10 & 2.53 & 26.8 & 9 & L & 17.5 & 16.2 & 7.12 & 865 & 1.51 & 100 & 0.04 & - & MRMW8S-W-30018 \\
\hline MW8S & $10-25$ & 04/20/11 & 2.73 & 26.8 & 9 & L & 17.5 & 10.1 & 6.88 & 652 & 0.30 & 98 & 0.00 & - & MRMW8S-W-30046 \\
\hline MW8S & $10-25$ & $10 / 04 / 11$ & 4.91 & 26.3 & 9 & L & 17.5 & 14.4 & 7.16 & 711 & 1.22 & 191 & 0.04 & - & MRMW8S-W-30075 \\
\hline MW8S & $10-25$ & $03 / 28 / 12$ & 1.38 & 26.8 & 6.25 & L & 17 & 13.6 & 6.63 & 708 & 0.51 & 111 & 0.02 & - & MRMW8S-W-30110 \\
\hline MW8S & $10-25$ & $05 / 07 / 13$ & 1.42 & 26.8 & 7 & L & 17.5 & 11.1 & 7.30 & 601 & 3.47 & 230 & 0.00 & - & MRMW8S-W-30147 \\
\hline MW8S & $10-25$ & 06/05/14 & 1.89 & 26.8 & 11 & L & 17.5 & 12.3 & 7.06 & 800 & 1.99 & 67 & 0.03 & - & MRMW8S-W-31952 \\
\hline MW8S & $10-25$ & $04 / 29 / 15$ & 4.7 & 26.8 & 9 & L & 17.5 & 14.5 & 7.16 & 862 & 5.11 & 152 & 0.01 & - & MRMW8S-W-37556 \\
\hline MW8S & $10-25$ & 05/06/16 & 0.85 & 26.8 & 8 & $\bar{L}$ & 17.5 & 13.9 & 7.01 & 917 & 4.05 & 176 & 0.05 & - & MRMW8S-W-39174 \\
\hline
\end{tabular}


TABLE 3.3 (Cont.)

\begin{tabular}{|c|c|c|c|c|c|c|c|c|c|c|c|c|c|c|c|}
\hline Location & $\begin{array}{l}\text { Screen } \\
\text { Interval } \\
\text { (ft BGL) }\end{array}$ & $\begin{array}{l}\text { Sample } \\
\text { Date }\end{array}$ & $\begin{array}{l}\text { Depth to } \\
\text { Water } \\
\text { (ft TOC) }\end{array}$ & $\begin{array}{l}\text { Depth of } \\
\text { Well } \\
\text { (ft TOC) }\end{array}$ & $\begin{array}{l}\text { Volume } \\
\text { Purged }\end{array}$ & $\begin{array}{l}\text { Purge } \\
\text { Units }\end{array}$ & $\begin{array}{l}\text { Pump } \\
\text { Intake } \\
\text { Position } \\
\text { (ft BGL) }\end{array}$ & $\begin{array}{c}\text { Temperature } \\
\left({ }^{\circ} \mathrm{C}\right)\end{array}$ & $\mathrm{pH}$ & $\begin{array}{c}\text { Conductivity } \\
(\mu \mathrm{S} / \mathrm{cm})\end{array}$ & $\begin{array}{l}\text { Dissolved } \\
\text { Oxygen } \\
\text { (mg/L) }\end{array}$ & $\begin{array}{l}\text { ORP } \\
(\mathrm{mV})\end{array}$ & $\begin{array}{l}\text { Iron II } \\
\text { (mg/L) }\end{array}$ & $\begin{array}{l}\text { Carbon } \\
\text { Dioxide } \\
(\mathrm{mg} / \mathrm{L})\end{array}$ & Sample \\
\hline MW8S & $10-25$ & 05/31/17 & 0.84 & 26.8 & 6 & L & 17.5 & 13.9 & 7.05 & 881 & 3.11 & 113 & 0.04 & - & MRMW8S-W-39797 \\
\hline MW9S & $38.83-53.83$ & 03/22/06 & 20.2 & 58.62 & 20 & gal & - & 14.6 & 7.17 & 715 & 0.41 & 25 & 0.00 & 35 & MRMW9S-W-20004 \\
\hline MW9S & $38.83-53.83$ & 09/19/06 & 18.87 & 59 & 22 & gal & - & 13.0 & 7.08 & 707 & 0.10 & 113 & 0.00 & 55 & MRMW9S-W-22494 \\
\hline MW9S & $38.83-53.83$ & 03/20/07 & 16.69 & 59 & 22 & gal & - & 14.2 & 6.39 & 714 & 0.21 & 40 & 0.00 & 20 & MRMW9S-W-16480 \\
\hline MW9S & $38.83-53.83$ & $10 / 01 / 07$ & 14 & 58.6 & 23 & gal & - & 15.5 & 7.05 & 664 & 5.50 & 191 & 0.00 & 30 & MRMW9S-W-16582 \\
\hline MW9S & $38.83-53.83$ & 04/14/08 & 16.58 & 58.63 & 2.29 & L & - & 12.6 & 7.33 & 709 & 1.93 & 266 & 0.07 & - & MRMW9S-W-23239 \\
\hline MW9S & $38.83-53.83$ & $10 / 20 / 08$ & 21.5 & 58.5 & 11 & $\mathrm{~L}$ & 46.33 & 15.1 & 7.15 & 690 & 6.18 & 106 & 0.00 & - & MRMW9S-W-27629 \\
\hline MW9S & $38.83-53.83$ & $04 / 23 / 09$ & 21.9 & 58.5 & 5.5 & L & 46.33 & 16.0 & 7.17 & 669 & 5.99 & -65 & 0.07 & - & MRMW9S-W-27661 \\
\hline MW9S & $38.83-53.83$ & 09/04/09 & 19.95 & 58.5 & 5 & L & 46.33 & 17.3 & 7.18 & 684 & 5.33 & 52 & 0.03 & - & MRMW9S-W-29951 \\
\hline MW9S & $38.83-53.83$ & 04/06/10 & 16.5 & 58.5 & 6 & L & 46.33 & 15.3 & 7.19 & 650 & 5.50 & -10 & 0.02 & - & MRMW9S-W-29991 \\
\hline MW9S & $38.83-53.83$ & 09/22/10 & 21.15 & 53.83 & 7 & $\mathrm{~L}$ & 46.33 & 17.1 & 7.12 & 704 & 6.07 & 151 & 0.00 & - & MRMW9S-W-30019 \\
\hline MW9S & $38.83-53.83$ & 04/20/11 & 23.76 & 58.32 & 14 & L & 46.33 & 13.6 & 7.07 & 569 & 5.42 & 111 & 0.00 & - & MRMW9S-W-30047 \\
\hline MW9S & $38.83-53.83$ & $10 / 04 / 11$ & 22.35 & 58.5 & 8 & $\mathrm{~L}$ & 46.33 & 16.7 & 7.14 & 618 & 5.90 & 185 & 0.05 & - & MRMW9S-W-30076 \\
\hline MW9S & $38.83-53.83$ & $03 / 28 / 12$ & 23.19 & 58.5 & 6.25 & L & 46.33 & 16.6 & 6.73 & 619 & 5.45 & 156 & - & - & MRMW9S-W-30111 \\
\hline MW9S & $38.83-53.83$ & $05 / 07 / 13$ & 23.48 & 58.5 & 7 & $\mathrm{~L}$ & 46.33 & 13.9 & 7.42 & 620 & 4.62 & 218 & 0.04 & - & MRMW9S-W-30148 \\
\hline MW9S & $38.83-53.83$ & 06/06/14 & 25.51 & 58.5 & 8 & L & 46.33 & 16.0 & 7.17 & 748 & 6.01 & 106 & 0.03 & - & MRMW9S-W-31953 \\
\hline MW9S & $38.83-53.83$ & 04/29/15 & 25.7 & 58.5 & 6 & L & 46.33 & 16.8 & 7.28 & 834 & 4.38 & 175 & 0.07 & - & MRMW9S-W-37557 \\
\hline MW9S & $38.83-53.83$ & $05 / 06 / 16$ & 19.00 & 58.5 & 7.25 & L & 46.33 & 15.8 & 7.11 & 791 & 6.36 & 177 & 0.00 & - & MRMW9S-W-39175 \\
\hline MW9S & $38.83-53.83$ & $05 / 31 / 17$ & 18.65 & 58.8 & 5.5 & L & 46.33 & 14.0 & 7.28 & 729 & 5.70 & 131 & 0.10 & - & MRMW9S-W-39798 \\
\hline MW10S & $30-45$ & 03/21/06 & 12.3 & 49.6 & 19 & gal & - & 6.3 & 7.11 & 701 & 2.10 & 88 & 0.01 & 40 & MRMW10S-W-19999 \\
\hline MW10S & $30-45$ & 09/18/06 & 11.08 & 49.61 & 20 & gal & - & 14.3 & 7.17 & 701 & 0.04 & 24 & 0.08 & 60 & MRMW10S-W-22489 \\
\hline MW10S & $30-45$ & $03 / 21 / 07$ & 10.77 & 49.61 & 20 & gal & - & 14.5 & 6.51 & 720 & 0.88 & 11 & 0.00 & 30 & MRMW10S-W-16485 \\
\hline MW10S & $30-45$ & $10 / 01 / 07$ & 6.95 & 49.65 & 20 & gal & - & 16.3 & 6.97 & 664 & 0.35 & 248 & 0.04 & 35 & MRMW10S-W-16593 \\
\hline MW10S & $30-45$ & $04 / 14 / 08$ & 9.82 & 49.7 & 1.9 & $\mathrm{~L}$ & - & 16.0 & 7.25 & 723 & 1.25 & 181 & 0.00 & - & MRMW10S-W-23240 \\
\hline MW10S & $30-45$ & $10 / 20 / 08$ & 13.7 & 49.7 & 5.4 & $\mathrm{~L}$ & 37.5 & 14.3 & 7.03 & 710 & 1.02 & 56 & 0.00 & - & MRMW10S-W-27630 \\
\hline MW10S & $30-45$ & $04 / 23 / 09$ & 13.6 & 45 & 7.5 & L & 37.5 & 15.1 & 7.05 & 668 & 1.78 & -57 & 0.07 & - & MRMW10S-W-27662 \\
\hline MW10S & $30-45$ & 09/03/09 & 12 & 49.7 & 7.5 & $\mathrm{~L}$ & 37.5 & 14.3 & 7.59 & 731 & 2.03 & 86 & 0.07 & - & MRMW10S-W-29952 \\
\hline MW10S & $30-45$ & 04/06/10 & 7.5 & 49.7 & 9 & $\mathrm{~L}$ & 37.5 & 16.1 & 7.06 & 649 & 2.91 & 221 & 0.00 & - & MRMW10S-W-29992 \\
\hline MW10S & $30-45$ & 09/22/10 & 11.69 & 49.7 & 11 & $\mathrm{~L}$ & 37.5 & 16.7 & 7.29 & 723 & 5.05 & 131 & 0.00 & - & MRMW10S-W-30020 \\
\hline MW10S & $30-45$ & 04/20/11 & 13.69 & 49.3 & 7.25 & L & 37.5 & 12.8 & 7.06 & 573 & 2.09 & 98.7 & 0.10 & - & MRMW10S-W-30048 \\
\hline MW10S & $30-45$ & $10 / 04 / 11$ & 12.54 & 49.7 & 8 & L & 37.5 & 15.4 & 7.27 & 613 & 2.53 & 201 & 0.01 & - & MRMW10S-W-30077 \\
\hline MW10S & $30-45$ & 03/28/12 & 12.76 & 49.7 & 8 & $\mathrm{~L}$ & 37.5 & 14.7 & 6.73 & 606 & 2.94 & 139 & 0.06 & - & MRMW10S-W-30112 \\
\hline MW10S & $30-45$ & 05/07/13 & 13.78 & 49.7 & 6 & $\mathrm{~L}$ & 37.5 & 16.9 & 7.33 & 602 & 2.24 & 195 & 0.00 & - & MRMW10S-W-30149 \\
\hline MW10S & $30-45$ & $06 / 05 / 14$ & 15.89 & 49.7 & 7 & $\mathrm{~L}$ & 37.5 & 13.9 & 7.20 & 701 & 2.49 & 45 & 0.03 & - & MRMW10S-W-31954 \\
\hline MW10S & $30-45$ & $04 / 29 / 15$ & 16.35 & 49.7 & 7 & L & 37.5 & 15.5 & 7.26 & 749 & 1.87 & 162 & 0.10 & - & MRMW10S-W-37558 \\
\hline MW10S & $30-45$ & $05 / 07 / 16$ & 8.25 & 49.7 & 7.75 & L & 37.5 & 15.4 & 7.11 & 823 & 2.41 & 152 & 0.13 & - & MRMW10S-W-39176 \\
\hline MW10S & $30-45$ & 06/01/17 & 8.13 & 49.7 & 6 & L & 37.5 & 14.4 & 7.18 & 778 & 2.76 & 210 & 0.11 & - & MRMW10S-W-39799 \\
\hline MW11S & $53-68$ & 03/22/06 & 35.2 & 72.5 & 20 & gal & - & 14.8 & 7.33 & 762 & 9.40 & 237 & 0.06 & 30 & MRMW11S-W-20001 \\
\hline MW11S & $53-68$ & 09/19/06 & 36 & 73.14 & 20 & gal & - & 13.0 & 7.24 & 764 & 1.42 & 158 & 0.02 & 30 & MRMW11S-W-22491 \\
\hline MW11S & $53-68$ & 03/20/07 & 34.65 & 73.14 & 20 & gal & - & 14.6 & 6.33 & 782 & 3.90 & 76 & 0.00 & 30 & MRMW11S-W-16479 \\
\hline MW11S & $53-68$ & 10/01/07 & 31.55 & 73 & 20 & gal & - & 16.4 & 6.49 & 624 & 6.57 & 241 & 0.04 & 35 & MRMW11S-W-16594 \\
\hline MW11S & $53-68$ & $04 / 15 / 08$ & 29.9 & 72.7 & 5.5 & L & - & 13.9 & 7.30 & 785 & 6.14 & 152 & 0.00 & - & MRMW11S-W-23241 \\
\hline MW11S & $53-68$ & 04/22/08 & 30.2 & 72.7 & 7.2 & L & - & 15.1 & 7.25 & 790 & 6.22 & 163 & - & - & MRMW11S-W-23261 \\
\hline MW11S & $53-68$ & $10 / 20 / 08$ & 37.1 & 72.7 & 9 & L & 60.5 & 14.3 & 7.16 & 756 & 8.95 & 104 & 0.00 & - & MRMW11S-W-27631 \\
\hline MW11S & $53-68$ & $10 / 21 / 08$ & - & - & TWV & - & - & 14.8 & 7.19 & 766 & - & - & 0.00 & - & MRMW11S-W-27651 \\
\hline
\end{tabular}


TABLE 3.3 (Cont.)

\begin{tabular}{|c|c|c|c|c|c|c|c|c|c|c|c|c|c|c|c|}
\hline Location & $\begin{array}{l}\text { Screen } \\
\text { Interval } \\
\text { (ft BGL) }\end{array}$ & $\begin{array}{l}\text { Sample } \\
\text { Date }\end{array}$ & $\begin{array}{l}\text { Depth to } \\
\text { Water } \\
\text { (ft TOC) }\end{array}$ & $\begin{array}{l}\text { Depth of } \\
\text { Well } \\
\text { (ft TOC) }\end{array}$ & $\begin{array}{l}\text { Volume } \\
\text { Purged }\end{array}$ & $\begin{array}{l}\text { Purge } \\
\text { Units }\end{array}$ & $\begin{array}{l}\text { Pump } \\
\text { Intake } \\
\text { Position } \\
\text { (ft BGL) }\end{array}$ & $\begin{array}{c}\text { Temperature } \\
\left({ }^{\circ} \mathrm{C}\right)\end{array}$ & $\mathrm{pH}$ & $\begin{array}{c}\text { Conductivity } \\
(\mu \mathrm{S} / \mathrm{cm})\end{array}$ & $\begin{array}{c}\text { Dissolved } \\
\text { Oxygen } \\
(\mathrm{mg} / \mathrm{L})\end{array}$ & $\begin{array}{l}\text { ORP } \\
(\mathrm{mV})\end{array}$ & $\begin{array}{l}\text { Iron II } \\
(\mathrm{mg} / \mathrm{L})\end{array}$ & $\begin{array}{l}\text { Carbon } \\
\text { Dioxide } \\
\text { (mg/L) }\end{array}$ & Sample \\
\hline MW11S & $53-68$ & $04 / 23 / 09$ & 38.1 & 72.7 & 5 & L & 60.5 & 16.5 & 7.19 & 722 & 9.03 & -62 & 0.09 & - & MRMW11S-W-27663 \\
\hline MW11S & $53-68$ & 09/03/09 & 34.7 & 72.7 & 7.5 & L & 60.5 & 13.9 & 7.63 & 777 & 9.35 & 102 & 0.05 & - & MRMW11S-W-29953 \\
\hline MW11S & $53-68$ & $09 / 04 / 09$ & 34.95 & 72.7 & 72 & L & 67 & 16.2 & 6.69 & 721 & 8.78 & 100 & - & - & MRMW11S-W-29973 \\
\hline MW11S & $53-68$ & $04 / 06 / 10$ & 29.45 & 72.7 & 6.5 & L & 60.5 & 15.0 & 7.14 & 700 & 7.20 & -20 & 0.00 & - & MRMW11S-W-29993 \\
\hline MW11S & $53-68$ & 09/23/10 & 34.75 & 72.7 & 7 & L & 60.5 & 15.7 & 7.40 & 756 & 12.62 & 179 & 0.33 & - & MRMW11S-W-30021 \\
\hline MW11S & $53-68$ & 04/20/11 & 39.16 & 72.5 & 7.5 & L & 60.5 & 13.1 & 7.03 & 603 & 7.20 & 108 & 0.00 & - & MRMW11S-W-30049 \\
\hline MW11S & $53-68$ & $10 / 04 / 11$ & 36.83 & 72.7 & 6 & L & 60.5 & 16.1 & 7.26 & 647 & 7.56 & 202 & 0.03 & - & MRMW11S-W-30078 \\
\hline MW11S & $53-68$ & $03 / 28 / 12$ & 36.46 & 72.7 & 6 & L & 60.5 & 15.6 & 6.73 & 641 & 7.37 & 158 & 0.00 & - & MRMW11S-W-30113 \\
\hline MW11S & $53-68$ & $05 / 07 / 13$ & 38.46 & 72.7 & 6 & L & 60.5 & 14.4 & 7.37 & 583 & 5.94 & 218 & 0.00 & - & MRMW11S-W-30150 \\
\hline MW11S & $53-68$ & 06/05/14 & 41.21 & 72.7 & 7 & L & 60.5 & 13.9 & 7.23 & 712 & 8.02 & 93 & 0.09 & - & MRMW11S-W-31955 \\
\hline MW11S & $53-68$ & 05/06/15 & 40.9 & 72.7 & 6 & L & 60.5 & 16.7 & 7.19 & 751 & 5.90 & 219 & 0.03 & - & MRMW11S-W-37559 \\
\hline MW11S & $53-68$ & $05 / 06 / 16$ & 29.75 & 72.7 & 8 & L & 60.5 & 15.3 & 7.08 & 800 & 7.69 & 182 & 0.02 & _- & MRMW11S-W-39177 \\
\hline MW11S & $53-68$ & $06 / 02 / 17$ & 30.06 & 72.7 & 5.5 & $\mathrm{~L}$ & 60.5 & 15.2 & 7.21 & 757 & 4.90 & 171 & 0.08 & - & MRMW11S-W-39800 \\
\hline Isch & _- & $02 / 19 / 04$ & _ & _ & Pump ${ }^{e}$ & _ & _ & _- & _- & _- & _ & _- & _- & _- & MRJR-W-16502 \\
\hline Isch & - & 09/14/05 & - & - & Pump & - & - & 20.4 & 6.73 & 2300 & - & - & - & - & MRPRISCH-W-16513 \\
\hline Isch & - & $03 / 23 / 06$ & - & - & 20 & gal & - & 13.0 & 7.23 & 9400 & - & - & - & - & MRISCH-W-19989 \\
\hline Isch & - & 09/19/06 & - & - & Pump & - & - & - & - & - & - & _- & _- & - & MRISCH-W-16531 \\
\hline Isch & - & $03 / 22 / 07$ & - & - & Pump & - & - & - & - & - & - & - & - & - & MRISCH-W-16564 \\
\hline Isch & - & $10 / 03 / 07$ & - & - & Pump & - & - & - & - & - & - & - & - & - & MRISCH-W-16590 \\
\hline Isch & - & $04 / 15 / 08$ & - & - & Pump & - & - & 12.6 & 7.33 & 3160 & - & - & 0.28 & - & MRISCH-W-23242 \\
\hline Isch & - & $10 / 21 / 08$ & - & - & Pump & - & - & - & - & - & - & - & - & - & MRISCH-W-27632 \\
\hline Isch & - & $04 / 22 / 09$ & - & - & Pump & - & - & 15.3 & 6.70 & 2389 & - & - & 0.04 & - & MRISCH-W-27664 \\
\hline Isch & - & $09 / 02 / 09$ & - & - & Pump & - & - & 13.9 & 7.18 & 2600 & - & _- & - & - & MRISCH-W-29954 \\
\hline Isch & - & $04 / 07 / 10$ & - & - & Pump & - & - & 11.8 & 6.86 & 2326 & - & - & 0.05 & - & MRISCH-W-29994 \\
\hline Isch & _- & $04 / 21 / 11$ & - & - & Pump & - & _- & 13.3 & 7.03 & 1949 & _- & _- & 0.35 & _- & MRISCH-W-30050 \\
\hline Isch & - & $10 / 03 / 11$ & - & - & Pump & - & - & 14.5 & 8.10 & 2011 & - & - & 1.61 & - & MRIsch-W-30079 \\
\hline Isch & - & $03 / 28 / 12$ & - & - & Pump & - & - & 15.5 & 7.12 & 2210 & - & - & 0.00 & - & MRISCH-W-30114 \\
\hline Isch & - & $05 / 07 / 13$ & - & - & Pump & - & - & 15.2 & 7.24 & 2054 & 5.38 & -11 & 0.26 & - & MRISCH-W-30151 \\
\hline Isch & - & $06 / 06 / 14$ & - & - & Pump & - & - & 15.9 & 7.10 & 2293 & 3.20 & 38 & 0.08 & - & MRISCH-W-31956 \\
\hline Isch & - & 04/30/15 & - & - & Pump & - & - & 15.8 & 7.05 & 2613 & 4.61 & 91 & 0.11 & - & MRISCH-W-37560 \\
\hline Rillinger & _- & $06 / 04 / 04$ & _- & _- & Pump & _- & _- & 15.9 & 6.99 & 2450 & _- & _- & _- & _- & MRPRIVRIL-W-16471 \\
\hline Rillinger & - & 09/14/05 & - & - & Pump & - & - & - & - & 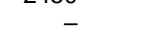 & - & - & _- & - & MRPRILL-W-16512 \\
\hline Rillinger & _- & 03/19/06 & - & _- & Pump & _- & _- & 11.9 & 7.05 & 2550 & _- & _ & _- & - & MRRILINGER-W-19988 \\
\hline Rillinger & - & 09/19/06 & - & - & Pump & _- & - & - & - & 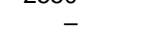 & - & - & - & - & MRRILI-W-16530 \\
\hline Rillinger & - & 03/29/07 & - & - & Pump & - & - & - & - & - & - & - & - & - & MRRILINGER-W-16561 \\
\hline Rillinger & - & $10 / 03 / 07$ & - & - & Pump & - & - & - & - & - & - & - & - & - & MRRILINGER-W-16591 \\
\hline Rillinger & - & $01 / 11 / 08$ & - & - & Pump & - & - & 12.2 & 7.46 & 884 & - & - & - & - & MORIL-W-11108 \\
\hline Rillinger & - & $04 / 15 / 08$ & - & - & Pump & - & - & 12.0 & 7.56 & 868 & - & - & 0.00 & - & MRRILINGER-W-23243 \\
\hline Rillinger & - & $10 / 21 / 08$ & - & - & Pump & - & - & - & - & - & - & - & - & - & MRRILLINGER-W-27633 \\
\hline Rillinger & - & $04 / 22 / 09$ & - & - & Pump & - & - & 14.5 & 7.14 & 2279 & - & - & 0.01 & - & MRRILLINGER-W-27665 \\
\hline Rillinger & - & $09 / 02 / 09$ & - & - & Pump & - & - & 14.8 & 7.60 & 809 & - & - & - & - & MRRILLINGER-W-29955 \\
\hline Rillinger & _- & $04 / 07 / 10$ & - & _- & Pump & _- & _- & 13.1 & 7.08 & 2287 & _- & _ & _- & - & MRRILLINGER-W-29995 \\
\hline Rillinger & - & $09 / 22 / 10$ & - & - & Pump & - & - & 14.9 & 7.04 & 2337 & - & - & 0.07 & - & MRRILLINGER-W-30023 \\
\hline Rillinger & - & $04 / 21 / 11$ & - & - & Pump & - & - & 13.5 & 7.15 & 1851 & - & - & - & - & MRRILLINGER-W-30051 \\
\hline Rillinger & - & $10 / 03 / 11$ & - & - & Pump & - & - & 14.4 & 7.37 & 1944 & - & - & 0.00 & - & MRRillinger-W-30080 \\
\hline Rillinger & _- & $03 / 28 / 12$ & - & _- & Pump & _- & _- & 14.6 & 7.10 & 1950 & - & - & 0.00 & - & MRRILLINGER-W-30115 \\
\hline
\end{tabular}


TABLE 3.3 (Cont.)

Footnotes on following page. 
a Not measured.

b TWV, three well volumes.

c PDS, purged dry and then sampled.

d NS, insufficient water to collect a sample.

e The well's pump was used for sampling. The pump was allowed to run before the sample was collected.

f Total depth. 
TABLE 3.4 Results of analyses at the AGEM Laboratory for VOCs in surface water and sediment samples collected in 2007-2016. ${ }^{a}$

\begin{tabular}{|c|c|c|c|c|c|c|}
\hline \multirow[b]{2}{*}{ Location } & \multirow[b]{2}{*}{ Sample } & \multirow[b]{2}{*}{$\begin{array}{l}\text { Sample } \\
\text { Date }\end{array}$} & \multirow[b]{2}{*}{ Medium } & \multicolumn{3}{|c|}{$\begin{array}{c}\text { Concentration ( } \mu \mathrm{g} / \mathrm{L} \text { in water; } \\
\mu \mathrm{g} / \mathrm{kg} \text { in sediment) }\end{array}$} \\
\hline & & & & $\begin{array}{c}\text { Carbon } \\
\text { Tetrachloride }\end{array}$ & Chloroform & $\begin{array}{l}\text { Methylene } \\
\text { Chloride }\end{array}$ \\
\hline SM1 & MRSM1-W-16572 & $03 / 22 / 07$ & Water & $N D^{b}$ & ND & ND \\
\hline SM1 & MRSM1-S-16573 & $03 / 22 / 07$ & Sediment & ND & ND & ND \\
\hline SM1 & MRSM1-W-16583 & $10 / 08 / 07$ & Water & ND & ND & ND \\
\hline SM1 & MRSM1-S-16584 & $10 / 08 / 07$ & Sediment & ND & ND & ND \\
\hline SM1 & MRSM1-W-23254 & $04 / 14 / 08$ & Water & ND & ND & ND \\
\hline SM1 & MRSM1-S-23254 & $04 / 14 / 08$ & Sediment & ND & ND & ND \\
\hline SM1 & MRSM1-W-27644 & $10 / 20 / 08$ & Water & ND & ND & ND \\
\hline SM1 & MRSM1-S-27644 & $10 / 20 / 08$ & Sediment & ND & ND & ND \\
\hline SM1 & MRSM1-W-27676 & $04 / 22 / 09$ & Water & ND & ND & ND \\
\hline SM1 & MRSM1-S-27676 & $04 / 22 / 09$ & Sediment & ND & ND & ND \\
\hline SM1 & MRSM1-W-29966 & 09/02/09 & Water & ND & ND & ND \\
\hline SM1 & MRSM1-S-29966 & $09 / 02 / 09$ & Sediment & ND & ND & ND \\
\hline SM1 & MRSM1-W-29974 & 04/06/10 & Water & ND & ND & ND \\
\hline SM1 & MRSM1-S-29974 & $04 / 06 / 10$ & Sediment & ND & ND & ND \\
\hline SM1 & MRSM1-W-30005 & $09 / 22 / 10$ & Water & ND & ND & ND \\
\hline SM1 & MRSM1-S-30005 & $09 / 22 / 10$ & Sediment & ND & ND & ND \\
\hline SM1 & MRSM1-W-29974 & $04 / 06 / 10$ & Water & ND & ND & ND \\
\hline SM1 & MRSM1-S-29974 & 04/06/10 & Sediment & ND & ND & ND \\
\hline SM1 & MRSM1-W-30062 & $10 / 03 / 11$ & Water & ND & ND & ND \\
\hline SM1 & MRSM1-S-30062 & $10 / 03 / 11$ & Sediment & ND & ND & ND \\
\hline SM1 & MRSM1-W-30094 & $03 / 27 / 12$ & Water & ND & ND & ND \\
\hline SM1 & MRSM1-S-30094 & $03 / 27 / 12$ & Sediment & ND & ND & ND \\
\hline SM1 & MRSM1-W-30131 & $05 / 06 / 13$ & Water & ND & ND & ND \\
\hline SM1 & MRSM1-S-30131 & $05 / 06 / 13$ & Sediment & ND & ND & ND \\
\hline SM1 & MRSM1-W-31920 & $06 / 06 / 14$ & Water & ND & ND & ND \\
\hline SM1 & MRSM1-S-31920 & $06 / 06 / 14$ & Sediment & ND & ND & ND \\
\hline SM1 & MRSM1-W-37524 & $04 / 28 / 15$ & Water & ND & ND & ND \\
\hline SM1 & MRSM1-S-37577 & 05/05/15 & Sediment & ND & ND & ND \\
\hline SM1 & MRSM1-W-39141 & $05 / 05 / 16$ & Water & ND & ND & ND \\
\hline SM1 & MRSM1-S-39141 & 05/0516 & Sediment & ND & ND & ND \\
\hline $\mathrm{SM} 2$ & MRSM2-W-16574 & 03/22/07 & Water & ND & ND & ND \\
\hline $\mathrm{SM} 2$ & MRSM2-S-16575 & $03 / 22 / 07$ & Sediment & ND & ND & ND \\
\hline SM2 & MRSM2-W-16585 & $10 / 08 / 07$ & Water & ND & ND & ND \\
\hline SM2 & MRSM2-S-16586 & $10 / 08 / 07$ & Sediment & ND & ND & ND \\
\hline SM2 & MRSM2-W-23255 & 04/14/08 & Water & ND & ND & ND \\
\hline SM2 & MRSM2-S-23255 & $04 / 14 / 08$ & Sediment & ND & ND & ND \\
\hline $\mathrm{SM} 2$ & MRSM2-W-27645 & $10 / 20 / 08$ & Water & ND & ND & ND \\
\hline SM2 & MRSM2-S-27645 & $10 / 20 / 08$ & Sediment & ND & ND & ND \\
\hline $\mathrm{SM} 2$ & MRSM2-W-27677 & 04/22/09 & Water & ND & ND & ND \\
\hline SM2 & MRSM2-S-27677 & $04 / 22 / 09$ & Sediment & ND & ND & ND \\
\hline $\mathrm{SM} 2$ & MRSM2-W-29967 & $09 / 02 / 09$ & Water & ND & ND & ND \\
\hline $\mathrm{SM} 2$ & MRSM2-S-29967 & 09/02/09 & Sediment & ND & ND & ND \\
\hline SM2 & MRSM2-W-29975 & 04/06/10 & Water & ND & ND & ND \\
\hline SM2 & MRSM2-S-29975 & 04/06/10 & Sediment & ND & ND & ND \\
\hline SM2 & MRSM2-W-30006 & $09 / 22 / 10$ & Water & ND & ND & ND \\
\hline SM2 & MRSM2-S-30006 & $09 / 22 / 10$ & Sediment & ND & ND & ND \\
\hline $\mathrm{SM} 2$ & MRSM2-W-29975 & 04/06/10 & Water & ND & ND & ND \\
\hline $\mathrm{SM} 2$ & MRSM2-S-29975 & 04/06/10 & Sediment & ND & ND & ND \\
\hline $\mathrm{SM} 2$ & MRSM2-W-30063 & $10 / 03 / 11$ & Water & ND & ND & ND \\
\hline SM2 & MRSM2-S-30063 & $10 / 03 / 11$ & Sediment & ND & ND & ND \\
\hline
\end{tabular}


TABLE 3.4 (Cont.)

\begin{tabular}{|c|c|c|c|c|c|c|}
\hline \multirow[b]{2}{*}{ Location } & \multirow[b]{2}{*}{ Sample } & \multirow[b]{2}{*}{$\begin{array}{l}\text { Sample } \\
\text { Date }\end{array}$} & \multirow[b]{2}{*}{ Medium } & \multicolumn{3}{|c|}{$\begin{array}{c}\text { Concentration ( } \mu \mathrm{g} / \mathrm{L} \text { in water; } \\
\mu \mathrm{g} / \mathrm{kg} \text { in sediment) }\end{array}$} \\
\hline & & & & $\begin{array}{l}\text { Carbon } \\
\text { Tetrachloride }\end{array}$ & Chloroform & $\begin{array}{l}\text { Methylene } \\
\text { Chloride }\end{array}$ \\
\hline SM2 & MRSM2-W-30095 & 03/27/12 & Water & ND & ND & ND \\
\hline SM2 & MRSM2-S-30095 & 03/27/12 & Sediment & ND & ND & ND \\
\hline SM2 & MRSM2-W-30132 & 05/06/13 & Water & ND & ND & ND \\
\hline SM2 & MRSM2-S-30132 & 05/06/13 & Sediment & ND & ND & ND \\
\hline SM2 & MRSM2-W-31921 & $06 / 06 / 14$ & Water & ND & ND & ND \\
\hline SM2 & MRSM2-S-31921 & $06 / 06 / 14$ & Sediment & ND & ND & ND \\
\hline SM2 & MRSM2-W-37525 & 04/28/15 & Water & ND & ND & ND \\
\hline SM2 & MRSM2-S-37578 & $05 / 05 / 15$ & Sediment & ND & ND & ND \\
\hline SM2 & MRSM2-W-39142 & $05 / 05 / 16$ & Water & ND & ND & ND \\
\hline SM2 & MRSM2-S-39142 & 05/05/16 & Sediment & ND & ND & ND \\
\hline SM3 & MRSM3-W-16576 & 03/22/07 & Water & ND & ND & ND \\
\hline SM3 & MRSM3-S-16577 & 03/22/07 & Sediment & ND & ND & ND \\
\hline SM3 & MRSM3-W-16587 & $10 / 08 / 07$ & Water & ND & ND & ND \\
\hline SM3 & MRSM3-S-16588 & 10/08/07 & Sediment & ND & ND & ND \\
\hline SM3 & MRSM3-W-23256 & 04/14/08 & Water & ND & ND & ND \\
\hline SM3 & MRSM3-S-23256 & 04/14/08 & Sediment & ND & ND & ND \\
\hline SM3 & MRSM3-W-27646 & 10/20/08 & Water & ND & ND & ND \\
\hline SM3 & MRSM3-S-27646 & 10/20/08 & Sediment & ND & ND & ND \\
\hline SM3 & MRSM3-W-27678 & $04 / 22 / 09$ & Water & ND & ND & ND \\
\hline SM3 & MRSM3-S-27678 & $04 / 22 / 09$ & Sediment & ND & ND & ND \\
\hline SM3 & MRSM3-W-29968 & 09/02/09 & Water & ND & ND & ND \\
\hline SM3 & MRSM3-S-29968 & 09/02/09 & Sediment & ND & ND & ND \\
\hline SM3 & MRSM3-W-29976 & 04/06/10 & Water & ND & ND & ND \\
\hline SM3 & MRSM3-S-29976 & 04/06/10 & Sediment & ND & ND & ND \\
\hline SM3 & MRSM3-W-30007 & 09/22/10 & Water & ND & ND & ND \\
\hline SM3 & MRSM3-S-30007 & 09/22/10 & Sediment & ND & ND & ND \\
\hline SM3 & MRSM3-W-29976 & $04 / 06 / 10$ & Water & ND & ND & ND \\
\hline SM3 & MRSM3-S-29976 & 04/06/10 & Sediment & ND & ND & ND \\
\hline SM3 & MRSM3-W-30064 & 10/03/11 & Water & ND & ND & ND \\
\hline SM3 & MRSM3-S-30064 & 10/03/11 & Sediment & ND & ND & ND \\
\hline SM3 & MRSM3-W-30096 & 03/27/12 & Water & ND & ND & ND \\
\hline SM3 & MRSM3-S-30096 & 03/27/12 & Sediment & ND & ND & ND \\
\hline SM3 & MRSM3-W-30133 & 05/06/13 & Water & ND & ND & ND \\
\hline SM3 & MRSM3-S-30133 & 05/06/13 & Sediment & ND & ND & ND \\
\hline SM3 & MRSM3-W-31922 & $06 / 06 / 14$ & Water & ND & ND & ND \\
\hline SM3 & MRSM3-S-31922 & $06 / 06 / 14$ & Sediment & ND & ND & ND \\
\hline SM3 & MRSM3-W-37526 & 04/28/15 & Water & ND & ND & ND \\
\hline SM3 & MRSM3-S-37579 & 05/05/15 & Sediment & ND & ND & ND \\
\hline SM3 & MRSM3-W-39143 & 05/05/16 & Water & ND & ND & ND \\
\hline SM3 & MRSM3-S-39143 & 05/05/16 & Sediment & ND & ND & ND \\
\hline SM4 & MRSM4-W-16578 & $03 / 22 / 07$ & Water & ND & ND & ND \\
\hline SM4 & MRSM4-S-16579 & 03/22/07 & Sediment & ND & ND & ND \\
\hline SM4 & MRSM4-W-16589 & 10/08/07 & Water & ND & ND & ND \\
\hline SM4 & MRSM4-S-16590 & 10/08/07 & Sediment & ND & ND & ND \\
\hline SM4 & MRSM4-W-23257 & 04/14/08 & Water & ND & ND & ND \\
\hline SM4 & MRSM4-S-23257 & 04/14/08 & Sediment & ND & ND & ND \\
\hline SM4 & MRSM4-W-27647 & 10/20/08 & Water & ND & ND & ND \\
\hline SM4 & MRSM4-S-27647 & 10/20/08 & Sediment & ND & ND & ND \\
\hline SM4 & MRSM4-W-27679 & $04 / 22 / 09$ & Water & ND & ND & ND \\
\hline SM4 & MRSM4-S-27679 & 04/22/09 & Sediment & ND & ND & ND \\
\hline
\end{tabular}


TABLE 3.4 (Cont.)

\begin{tabular}{|c|c|c|c|c|c|c|}
\hline \multirow[b]{2}{*}{ Location } & \multirow[b]{2}{*}{ Sample } & \multirow[b]{2}{*}{$\begin{array}{l}\text { Sample } \\
\text { Date }\end{array}$} & \multirow[b]{2}{*}{ Medium } & \multicolumn{3}{|c|}{$\begin{array}{c}\text { Concentration ( } \mu \mathrm{g} / \mathrm{L} \text { in water; } \\
\mu \mathrm{g} / \mathrm{kg} \text { in sediment) }\end{array}$} \\
\hline & & & & $\begin{array}{c}\text { Carbon } \\
\text { Tetrachloride }\end{array}$ & Chloroform & $\begin{array}{c}\text { Methylene } \\
\text { Chloride }\end{array}$ \\
\hline SM4 & MRSM4-W-29969 & 09/02/09 & Water & ND & ND & ND \\
\hline SM4 & MRSM4-S-29969 & 09/02/09 & Sediment & ND & ND & ND \\
\hline SM4 & MRSM4-W-29977 & $04 / 06 / 10$ & Water & ND & ND & ND \\
\hline SM4 & MRSM4-S-29977 & $04 / 06 / 10$ & Sediment & ND & ND & ND \\
\hline SM4 & MRSM4-W-30008 & $09 / 22 / 10$ & Water & ND & ND & ND \\
\hline SM4 & MRSM4-S-30008 & $09 / 22 / 10$ & Sediment & ND & ND & ND \\
\hline SM4 & MRSM4-W-29977 & $04 / 06 / 10$ & Water & ND & ND & ND \\
\hline SM4 & MRSM4-S-29977 & $04 / 06 / 10$ & Sediment & ND & ND & ND \\
\hline SM4 & MRSM4-W-30065 & $10 / 03 / 11$ & Water & ND & ND & ND \\
\hline SM4 & MRSM4-S-30065 & $10 / 03 / 11$ & Sediment & ND & ND & ND \\
\hline SM4 & MRSM4-W-30097 & $03 / 27 / 12$ & Water & ND & ND & ND \\
\hline SM4 & MRSM4-S-30097 & $03 / 27 / 12$ & Sediment & ND & ND & ND \\
\hline SM4 & MRSM4-W-30134 & $05 / 06 / 13$ & Water & ND & ND & ND \\
\hline SM4 & MRSM4-S-30134 & $05 / 06 / 13$ & Sediment & $5.4 \mathrm{Jc}$ & ND & ND \\
\hline SM4 & MRSM4-W-31923 & $06 / 06 / 14$ & Water & ND & ND & ND \\
\hline SM4 & MRSM4-S-31923 & $06 / 06 / 14$ & Sediment & ND & ND & ND \\
\hline SM4 & MRSM4-W-37527 & $04 / 28 / 15$ & Water & ND & ND & ND \\
\hline SM4 & MRSM4-S-37580 & $05 / 05 / 15$ & Sediment & ND & ND & ND \\
\hline SM4 & MRSM4-W-37527 & $04 / 28 / 15$ & Water & ND & ND & ND \\
\hline SM4 & MRSM4-S-37580 & $05 / 05 / 15$ & Sediment & ND & ND & ND \\
\hline SM4 & MRSM4-W-39144 & $05 / 05 / 16$ & Water & ND & ND & ND \\
\hline SM4 & MRSM4-S-39144 & $05 / 05 / 16$ & Sediment & ND & ND & ND \\
\hline SMB & MRSMB-W-16570 & 03/22/07 & Water & ND & ND & ND \\
\hline SMB & MRSMB-S-16571 & $03 / 22 / 07$ & Sediment & ND & ND & ND \\
\hline SMB & MRSMB-W-16581 & $10 / 08 / 07$ & Water & ND & ND & ND \\
\hline SMB & MRSMB-S-16582 & $10 / 08 / 07$ & Sediment & ND & ND & ND \\
\hline SMB & MRSMB-W-23258 & $04 / 14 / 08$ & Water & ND & ND & ND \\
\hline SMB & MRSMB-S-23258 & $04 / 14 / 08$ & Sediment & ND & ND & ND \\
\hline SMB & MRSMB-W-27648 & $10 / 20 / 08$ & Water & ND & ND & ND \\
\hline SMB & MRSMB-S-27648 & $10 / 20 / 08$ & Sediment & ND & ND & ND \\
\hline SMB & MRSMB-W-27680 & $04 / 22 / 09$ & Water & ND & ND & ND \\
\hline SMB & MRSMB-S-27680 & $04 / 22 / 09$ & Sediment & ND & ND & ND \\
\hline SMB & MRSMB-W-29970 & 09/02/09 & Water & ND & ND & ND \\
\hline SMB & MRSMB-S-29970 & $09 / 02 / 09$ & Sediment & ND & ND & ND \\
\hline SMB & MRSMB-W-29978 & $04 / 06 / 10$ & Water & ND & ND & ND \\
\hline SMB & MRSMB-S-29978 & $04 / 06 / 10$ & Sediment & ND & ND & ND \\
\hline SMB & MRSMB-W-30009 & 09/22/10 & Water & ND & ND & ND \\
\hline SMB & MRSMB-S-30009 & $09 / 22 / 10$ & Sediment & ND & ND & ND \\
\hline SMB & MRSMB-W-29978 & $04 / 06 / 10$ & Water & ND & ND & ND \\
\hline SMB & MRSMB-S-29978 & $04 / 06 / 10$ & Sediment & ND & ND & ND \\
\hline SMB & MRSMB-W-30066 & $10 / 03 / 11$ & Water & ND & ND & ND \\
\hline SMB & MRSMB-S-30066 & $10 / 03 / 11$ & Sediment & ND & ND & ND \\
\hline SMB & MRSMB-W-30098 & $03 / 27 / 12$ & Water & ND & ND & ND \\
\hline SMB & MRSMB-S-30098 & $03 / 27 / 12$ & Sediment & ND & ND & ND \\
\hline SMB & MRSMB-W-30135 & $05 / 06 / 13$ & Water & ND & ND & ND \\
\hline SMB & MRSMB-S-30135 & $05 / 06 / 13$ & Sediment & ND & ND & ND \\
\hline SMB & MRSMB-W-31924 & $06 / 06 / 14$ & Water & ND & ND & ND \\
\hline SMB & MRSMB-S-31924 & $06 / 06 / 14$ & Sediment & ND & ND & ND \\
\hline SMB & MRSMB-W-37528 & $04 / 28 / 15$ & Water & ND & ND & ND \\
\hline
\end{tabular}


TABLE 3.4 (Cont.)

\begin{tabular}{|c|c|c|c|c|c|c|}
\hline \multirow[b]{2}{*}{ Location } & \multirow[b]{2}{*}{ Sample } & \multirow[b]{2}{*}{$\begin{array}{l}\text { Sample } \\
\text { Date }\end{array}$} & \multirow[b]{2}{*}{ Medium } & \multicolumn{3}{|c|}{$\begin{array}{c}\text { Concentration ( } \mu \mathrm{g} / \mathrm{L} \text { in water; } \\
\mu \mathrm{g} / \mathrm{kg} \text { in sediment) }\end{array}$} \\
\hline & & & & $\begin{array}{l}\text { Carbon } \\
\text { Tetrachloride }\end{array}$ & Chloroform & $\begin{array}{l}\text { Methylene } \\
\text { Chloride }\end{array}$ \\
\hline SMB & MRSMB-S-37581 & 05/05/15 & Sediment & ND & ND & ND \\
\hline SMB & MRSMB-W-39140 & 05/05/16 & Water & ND & ND & ND \\
\hline SMB & MRSMB-S-39140 & $05 / 05 / 15$ & Sediment & ND & ND & ND \\
\hline
\end{tabular}

a Analyses conducted at the AGEM Laboratory with EPA Method 524.2 for surface water samples or modified EPA Method 5030B/8260B for sediment samples.

b ND, compound analyzed for but not detected at a level greater than or equal to the method detection limit (<1 $\mu \mathrm{g} / \mathrm{L}$ for surface water samples; $10 \mu \mathrm{g} / \mathrm{kg}$ for sediment samples).

c J, compound identified at an estimated concentration between the instrument detection limit and the method detection limit. 
TABLE 3.5 Evaluation results for private wells in 2017.

\begin{tabular}{|c|c|c|c|c|c|c|}
\hline Well & $\begin{array}{l}\text { Depth } \\
\text { (ft BGL) }\end{array}$ & $\begin{array}{l}\text { Date of } \\
\text { Last } \\
\text { Sampling }\end{array}$ & $\begin{array}{l}\text { Owner on Date of } \\
\text { Last Sampling }\end{array}$ & $\begin{array}{l}\text { Owner during Report } \\
\text { Monitoring Period }\end{array}$ & Owner Address & Use and Condition \\
\hline Allen & - & $08 / 27 / 04$ & C.L. Allen & $\begin{array}{l}\text { No change in } \\
\text { ownership }\end{array}$ & $\begin{array}{l}90 \text { Roxanna St. } \\
\text { Morrill, KS } 66515\end{array}$ & $\begin{array}{l}\text { The hand pump on the hand-dug well has } \\
\text { not been functional for several years, and } \\
\text { the well has not been used for any purpose } \\
\text { for many years. The owner confirmed that } \\
\text { he has no plans to use the well again. }\end{array}$ \\
\hline $\begin{array}{l}\text { Edwards } \\
\text { (formerly } \\
\text { Manning) }\end{array}$ & - & $\begin{array}{l}\text { Never } \\
\text { sampled }\end{array}$ & D. and R. Manning & A. Edwards & $\begin{array}{l}207 \text { Roxanna St. } \\
\text { Morrill, KS } 66515\end{array}$ & $\begin{array}{l}\text { The property was purchased by A. Edwards, } \\
\text { who now resides in the home. The lawn and } \\
\text { garden well is hand-dug and operated by a } \\
\text { hand pump. The previous owners used the } \\
\text { well for only non-drinking purposes. }\end{array}$ \\
\hline $\begin{array}{l}\text { Gruber } \\
\text { (formerly } \\
\text { Manning/Cain) }\end{array}$ & - & 06/04/04 & T. Gruber & $\begin{array}{l}\text { No change in } \\
\text { ownership }\end{array}$ & $\begin{array}{l}202 \text { Roxanna St. } \\
\text { Morrill, KS } 66515\end{array}$ & $\begin{array}{l}\text { The home on the property was destroyed in } \\
\text { a fire and razed in early } 2015 \text {. T. Gruber } \\
\text { purchased in property in } 2015 \text { and removed } \\
\text { the fire debris. He stated that he has no } \\
\text { plans to build a new home on the property, } \\
\text { which remains a grass lot. There is no } \\
\text { visible sign of any well on the property. }\end{array}$ \\
\hline Rilinger & - & 06/01/17 & L. Rilinger & $\begin{array}{l}\text { No change in } \\
\text { ownership }\end{array}$ & $\begin{array}{l}106 \text { Walnut Street, } \\
\text { Morrill, KS } 66515\end{array}$ & $\begin{array}{l}\text { Well used by owner for lawn and garden } \\
\text { purposes. }\end{array}$ \\
\hline Stone & 43 & 06/01/17 & J. Stone & $\begin{array}{l}\text { No change in } \\
\text { ownership }\end{array}$ & $\begin{array}{l}102 \text { Virginia Street, } \\
\text { Morrill, KS } 66515^{a}\end{array}$ & $\begin{array}{l}\text { The well has not been used by the owner for } \\
\text { several years and lacks a pump. }\end{array}$ \\
\hline
\end{tabular}

a Reported address is the well's location. The owner's mailing address is 406 Walnut Street, Morrill, Kansas 66515. 


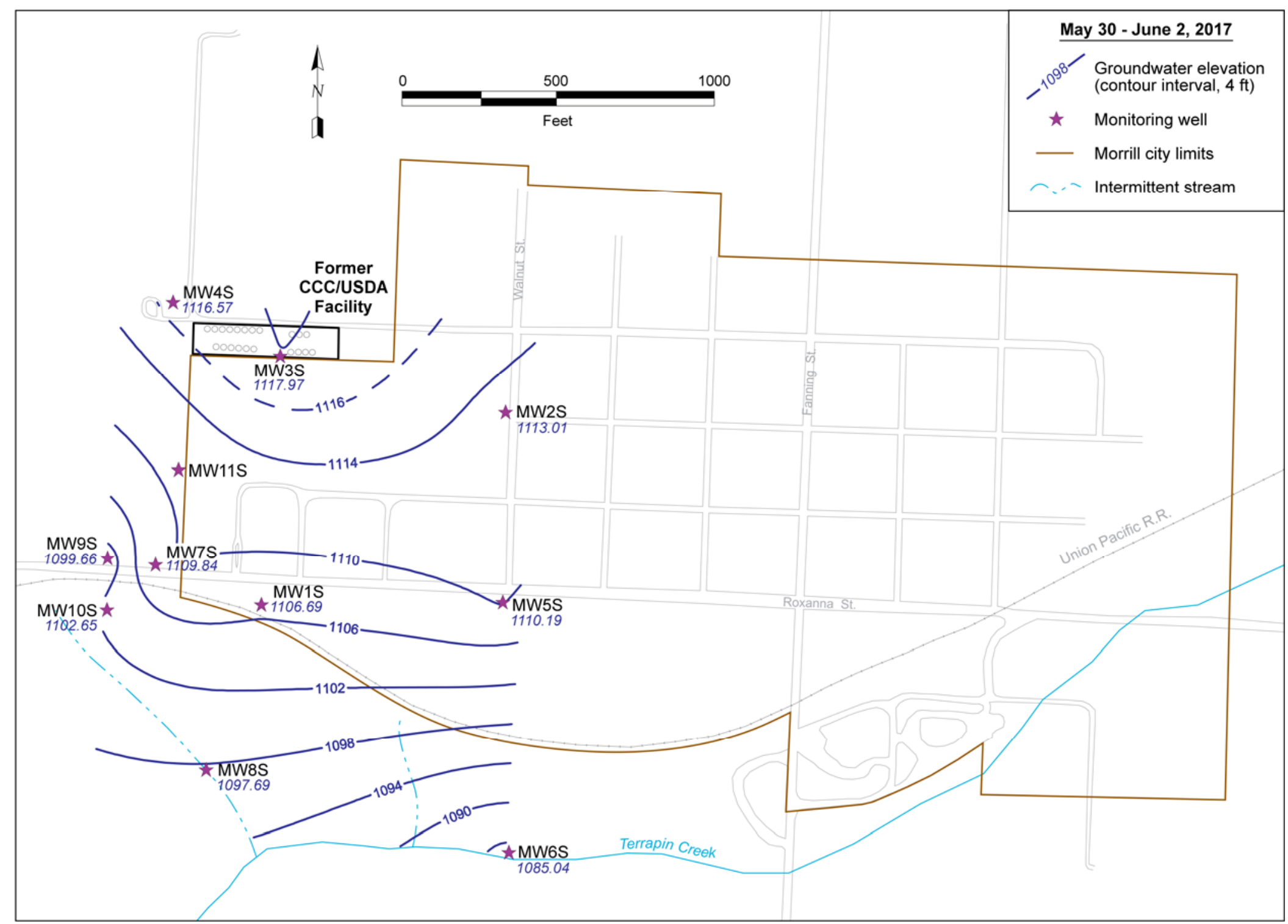

FIGURE 3.1 Potentiometric surface based on water levels measured manually on May 30-June 2, 2017. 


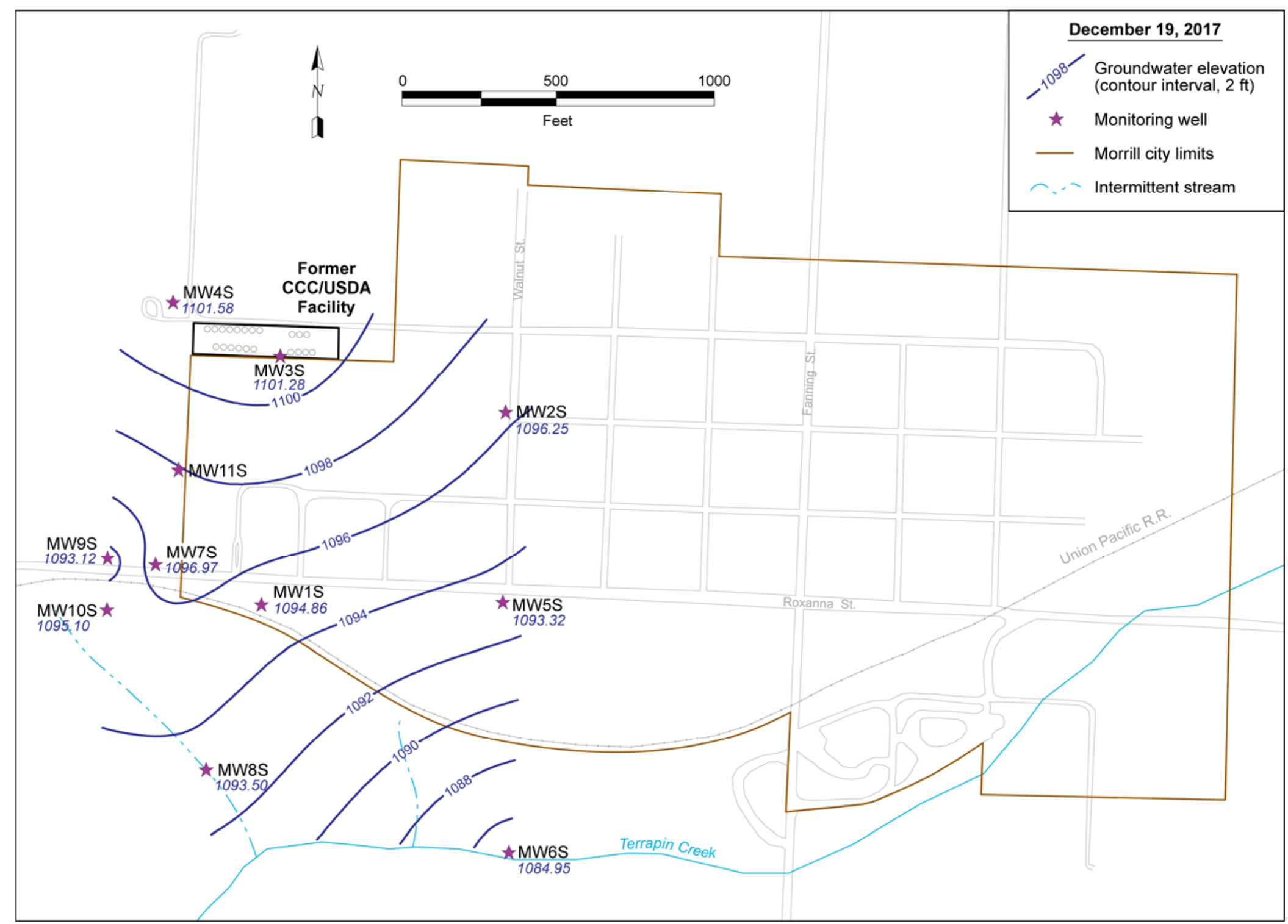

FIGURE 3.2 Potentiometric surface based on water levels measured manually on December 19, 2017. 
(1) $8 / 30 / 18$

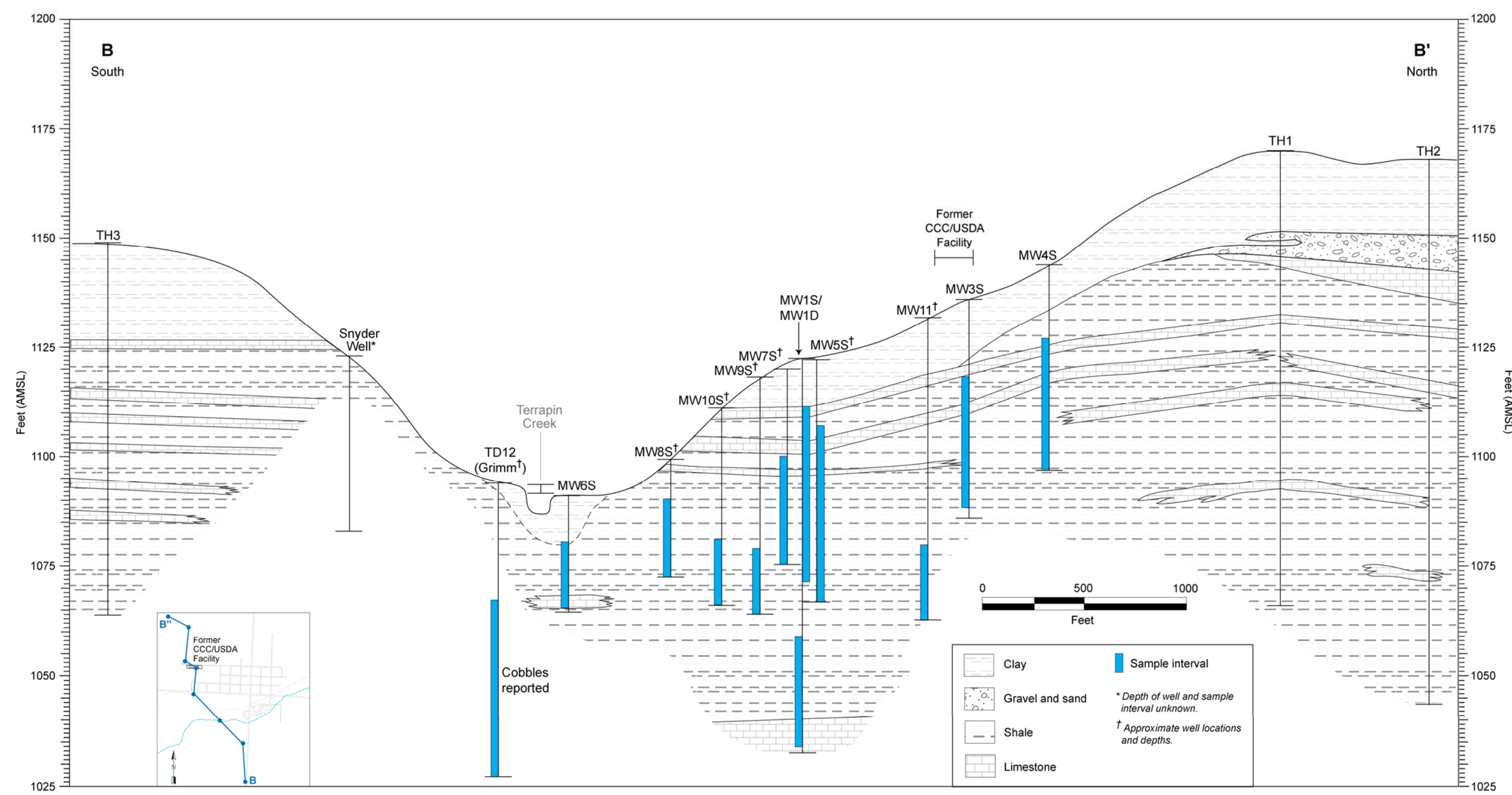

FIGURE 3.3 Interpretive south-north geologic section illustrating the stratigraphic relationships among screened intervals of wells near Terrapin Creek. 


\section{Morrill Water Levels \\ 2017}

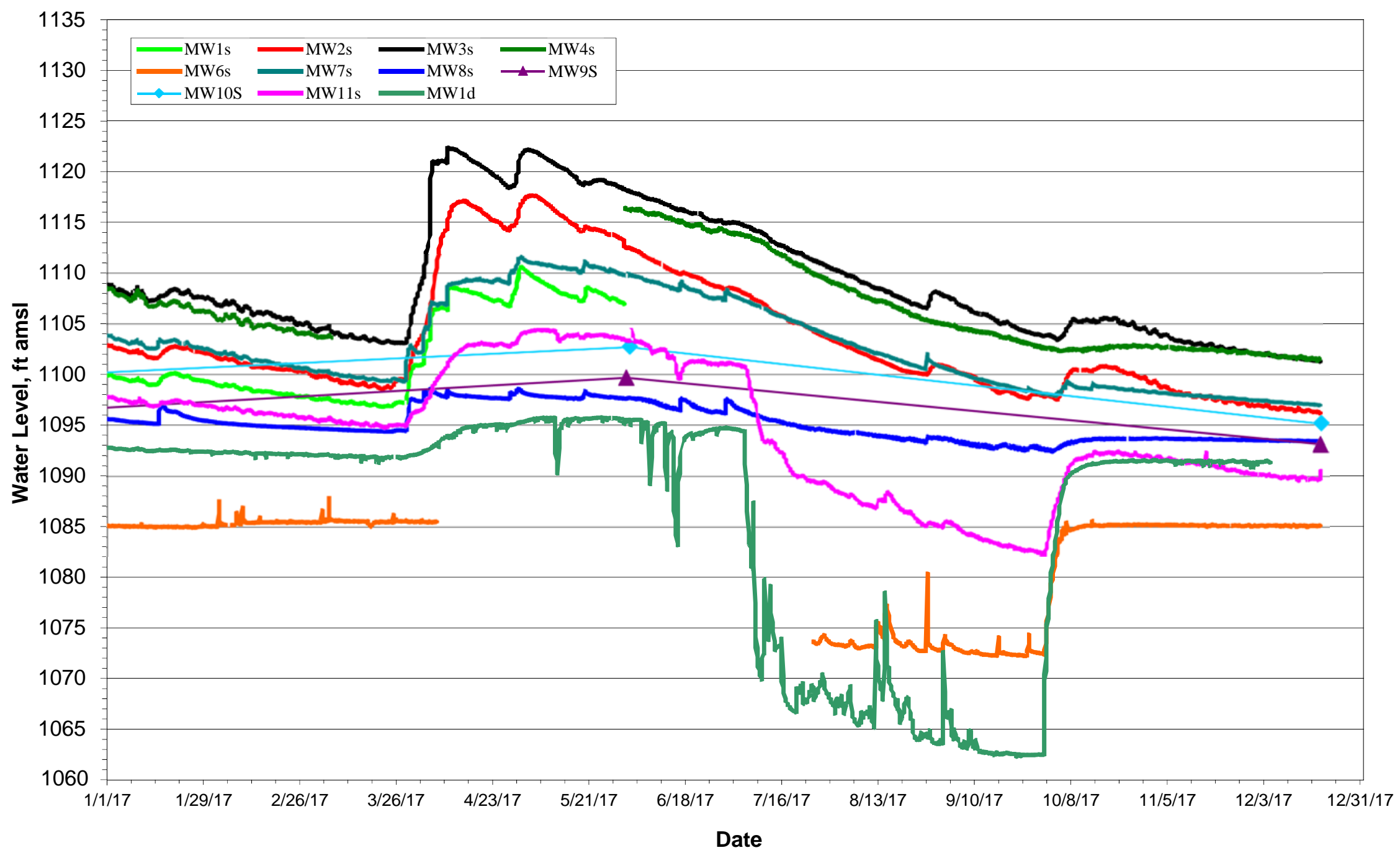

FIGURE 3.4 Hydrographs summarizing results of long-term water level monitoring in 2017. 


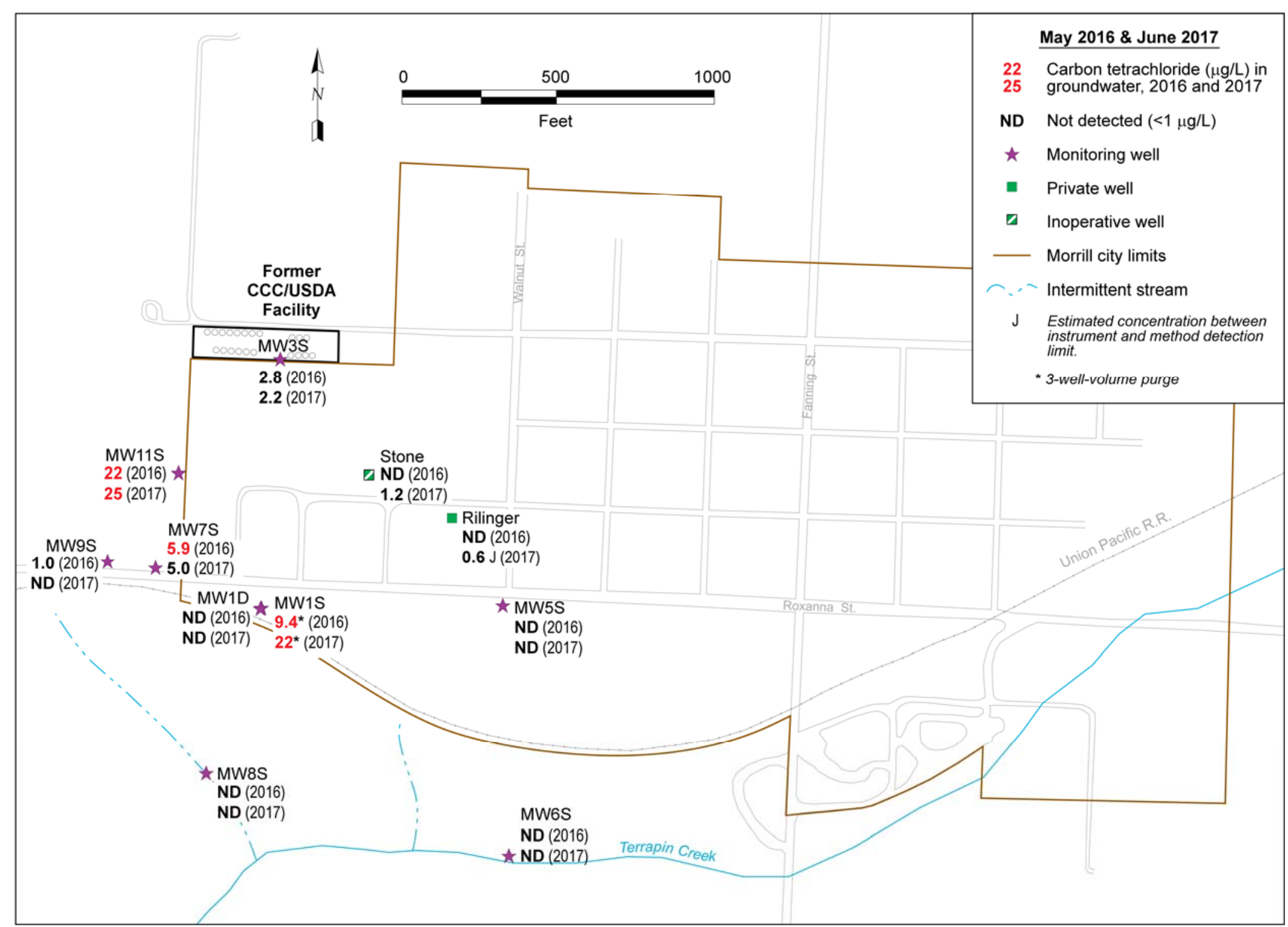

FIGURE 3.5 Carbon tetrachloride concentrations in groundwater, May 2016 and May-June 2017. 


\section{Conclusions, Future Activities, and Recommendations}

\subsection{Conclusions}

The findings of the May-June 2017 monitoring event at Morrill support the following conclusions:

- As in prior years, groundwater flow in 2017 was predominantly to the south, from the vicinity of the former CCC/USDA facility toward Terrapin Creek. Automatic water level monitoring data indicate that, historically, spring precipitation and recharge are the dominant factors affecting the local groundwater level patterns during much of the year.

- The hydrographs for MW1D, MW6S, and MW11S continue to show the clear influence of the Grimm irrigation well pumping in the stratigraphically deeper part of the aquifer during the late spring and summer. The water level recorder at MW11S was installed in late 2013 to help document this influence.

- The distribution of carbon tetrachloride in groundwater during the May-June 2017 monitoring event was generally consistent with that seen in the May 2016 monitoring event. In May-June 2017, a maximum carbon tetrachloride concentration of $25 \mu \mathrm{g} / \mathrm{L}$ was identified in groundwater at well MW11S, with concentrations decreasing downgradient toward Terrapin Creek. Chloroform concentrations were also consistent with recent previous values, with a maximum trace concentration of $<1 \mu \mathrm{g} / \mathrm{L}$ at well MW1S.

- The carbon tetrachloride concentration at the former CCC/USDA facility (MW3S, where the maximum level of $390 \mu \mathrm{g} / \mathrm{L}$ occurred in 1995) was notably lower in 2017 (2.2 $\mu \mathrm{g} / \mathrm{L})$ and 2016 (2.8 $\mu \mathrm{g} / \mathrm{L})$, compared with 2015 (38 $\mu \mathrm{g} / \mathrm{L})$. An increase in the carbon tetrachloride concentration observed at MW1S in $2016(9.4 \mu \mathrm{g} / \mathrm{L})$ persisted in $2017(22 \mu \mathrm{g} / \mathrm{L})$; the value in 2015 was $4.6 \mu \mathrm{g} / \mathrm{L}$.

- Since 2004, the accumulated results of many sampling events have demonstrated a significant decline in the maximum detected concentration of 
carbon tetrachloride in groundwater. In 1995, the maximum occurred at the former CCC/USDA facility (390 $\mu \mathrm{g} / \mathrm{L}$ at MW3S), whereas the maximum in 2017 was $25 \mu \mathrm{g} / \mathrm{L}$ (at MW11S). Chloroform concentrations have decreased from $9.6 \mu \mathrm{g} / \mathrm{L}$ in 1995 (at MW3S) to < $1 \mu \mathrm{g} / \mathrm{L}$ (at MW1S) in 2017. These are decreases of $94 \%$ for carbon tetrachloride and $>89 \%$ for chloroform. The changes in carbon tetrachloride concentrations at wells sampled in 1995-2017 are illustrated in Figure 4.1.

- The residual contaminant plume extending from the former CCC/USDA facility southward toward Terrapin Creek is well-defined and is slowly declining in concentration naturally (Sections 3.2 and 3.3).

- Since 2007, the accumulated results of many monitoring events for surface water and sediment in Terrapin Creek have demonstrated no impact to the sediment and surface waters of the creek by carbon tetrachloride, aside from a trace detection in 1 of 5 shallow streambed sediment samples collected in 2013. Risk for further degradation of the creek continues to be monitored.

- Terrapin Creek (tributary segment 308 to Walnut Creek) receives discharge from the Morrill wastewater treatment plant and several confined animal feeding operations regulated by the KDHE. The Walnut Creek watershed is designated by the KDHE as impaired by fecal coliform bacteria. Terrapin Creek is classified by the KDHE as not open to or accessible by the public for contact recreation; it does not support the food procurement designated use (KDHE 2010).

- Use of the public water supply wells that had served Morrill in 1922-1991 was terminated because of high nitrate levels and poor water quality. Public water was subsequently obtained from the Sabetha municipal water system.

- The evaluations of private wells indicated the following:

- T. Gruber continues to own the former Manning/Cain property. The area where the former home burned down is planted with grass. There is no visible sign of any well on the property. This is consistent with the statement 
by the previous owners (the Mannings), who maintained that they had no knowledge of the existence, location, or use of a well on this property. The current owner stated that he has no plans for the property other than to mow the grass cover.

- $\quad$ The Allen private well has not been in use for years and is not expected to be used in the future.

- The former Manning property was purchased by A. Edwards, who now resides in the home where the lawn and garden well is located. The well is used only for non-drinking purposes.

- The ownership, use, and condition of the Rilinger and Stone wells remain the same as reported in previous years (Table 3.5).

\subsection{Recommendation}

Given our current understanding of the size, nature, and behavior of the groundwater carbon tetrachloride plume, the CCC/USDA recommends the Morrill site for entry into KDHE's Risk Management Program (RMP) in accordance with the eligibility criteria in KDHE's Risk Management Program Guidance, Policy and Scope of Work, \#BER-RS-057 (KDHE 2016b). The following paragraphs discuss how the former CCC/USDA facility meets each of the criteria.

- $\quad$ Subject to an agreement or order under the authority of KDHE BER. The site investigation and subsequent KDHE-approved long-term monitoring program have been conducted as part of the Intergovernmental Agreement (IGA) between the KDHE and the CCC/USDA. The original agreement was signed in 1999, and has been renewed every three years since that time. Per the IGA, the KDHE provides technical oversight and review/approval of the investigation activities at former CCC/USDA facilities in Kansas. For the Morrill site, the CCC/USDA assumed responsibility for investigation of the carbon tetrachloride contamination in 2003. 
The CCC/USDA involvement began in 2003 with the development and implementation of a work plan for a Phase I site investigation. That investigation and subsequent investigations in 2004 and 2005 were approved by KDHE, per the IGA. The investigations were performed on behalf of the CCC/USDA by Argonne National Laboratory.

The initial (2003) investigation by the CCC/USDA determined that soils at the former facility were not affected by grain fumigation activities. Carbon tetrachloride was not detected in near surface soils or in subsurface soils collected to bedrock. Because no identifiable human health risk was associated with carbon tetrachloride in shallow soils, the conclusion was that no further threat of contamination to groundwater was present.

Per the KDHE, in September 2005, the CCC/USDA initiated periodic sampling of groundwater, in accord with a KDHE-approved groundwater monitoring program, to monitor carbon tetrachloride concentrations in the groundwater. Every year since the outset of the long-term monitoring program, the CCC/USDA has provided the KDHE with an annual groundwater monitoring report with the results of the long-term monitoring program. KDHE has approved all of the monitoring reports per the IGA.

- Poses a low risk to human health and the environment. Historic groundwater and soil characterization sampling of the former grain silo site and groundwater plume beginning in 1989, summarized in Section 1, combined with long-term annual groundwater sampling has established that carbon tetrachloride contamination is confined to a small, well-defined, low-level groundwater plume that poses low risk to human health and the environment. There are no drinking water wells within the plume; all residents of Morrill obtain their drinking water from a municipal water supply. Sampling of indoor air, surface water, and vegetation has established that no other exposure pathways are complete for carbon tetrachloride groundwater contamination.

- $\quad$ The extent of environmental contamination has been clearly defined. Long-term groundwater sampling of a monitoring well network that was designed in consideration of site history, release locations, and local stratigraphy and 
hydrology has clearly defined the extent and migration of groundwater contamination.

- Source reduction has been completed, if necessary. The original source of carbon tetrachloride contamination, the grain silos, has long since been removed. Initial investigations by the CCC/USDA of soils underlying and adjacent to the silos were completed in 2003 and found no residual carbon tetrachloride or its breakdown product chloroform in surface soils or subsurface soils from the surface down to bedrock (see Section 1).

- Contaminant concentration trends are not dependent on continued operation and maintenance of active remediation systems. There are no active remediation systems in place for the groundwater plume, nor are any needed to reduce plume levels. Plume concentrations are stable and decreasing overall as the plume migrates downgradient.

- The associated groundwater contaminant plume is stable or shrinking. As observed in long-term monitoring, the groundwater plume is stable and shrinking in a predictable manner.

- Imminent future exposure to contaminated media is not likely. As noted for the second criterion, there are no complete exposure pathways for human exposure to plume contaminants.

- All current complete exposure pathways have been addressed. There are no complete exposure pathways. The plume is not a source of drinking water. 


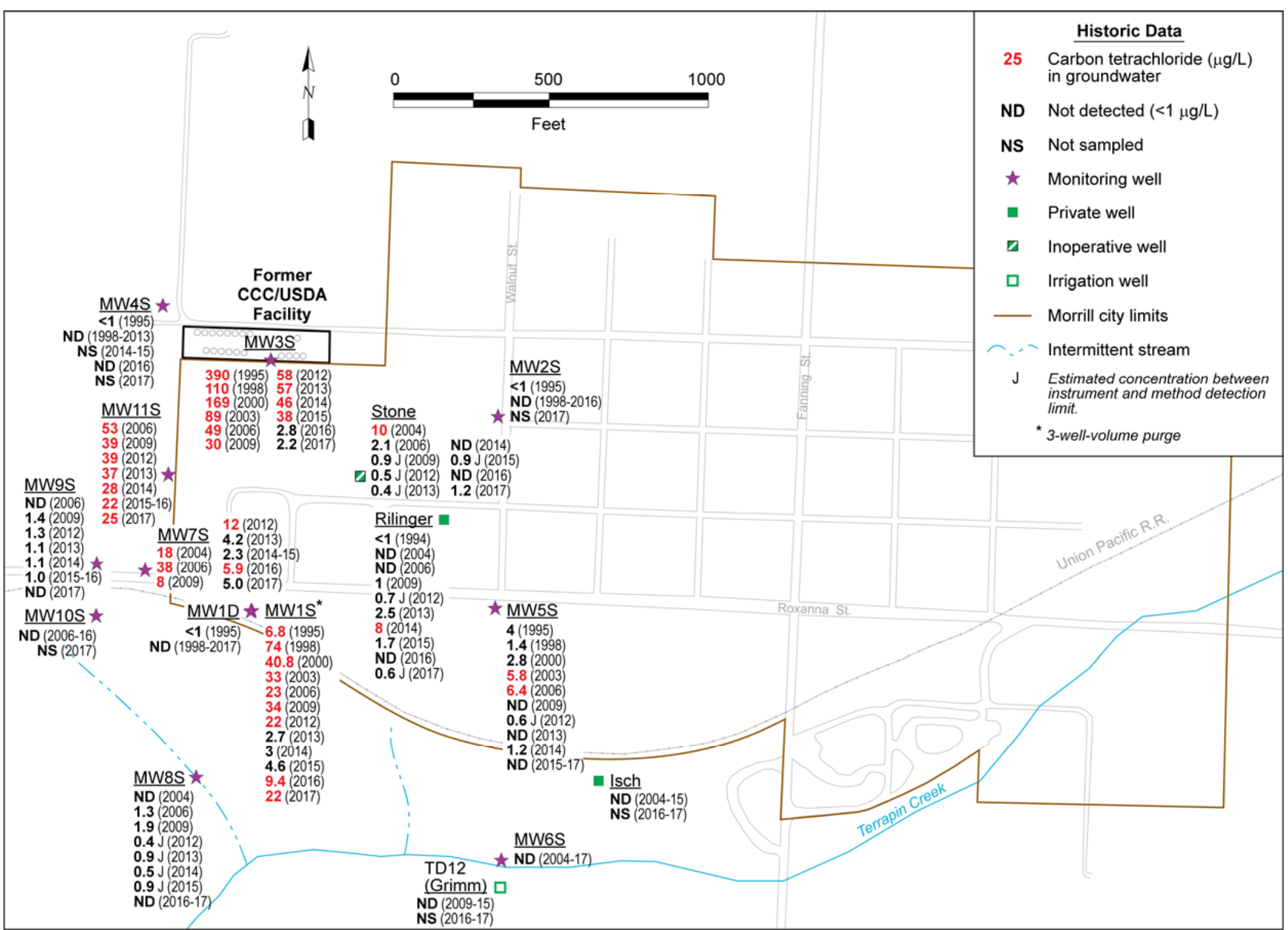

FIGURE 4.1 Historical carbon tetrachloride concentrations in groundwater, 1995-2017. 


\section{References}

Argonne, 2002, Final Master Work Plan: Environmental Investigations at Former CCC/USDA Facilities in Kansas, 2002 Revision, ANL/ER/TR-02/004, prepared for the Commodity Credit Corporation, U.S. Department of Agriculture, Washington, D.C., by Argonne National Laboratory, Argonne, Illinois, December.

Argonne, 2003, Final Work Plan: Phase I Expedited Site Characterization, Morrill, Kansas, ANL/ER/TR-03/001, prepared for the Commodity Credit Corporation, U.S. Department of Agriculture, Washington, D.C., by Argonne National Laboratory, Argonne, Illinois, September.

Argonne, 2004, Final Phase I-Phase II Interim Report: Expedited Site Characterization, Morrill, Kansas, ANL/ER/TR-04/001, prepared for the Commodity Credit Corporation, U.S. Department of Agriculture, Washington, D.C., by Argonne National Laboratory, Argonne, Illinois, December.

Argonne, 2005a, Final Report, Monitoring Well Installation and Sampling, 2004, at Morrill, Kansas, ANL/ER/TR-04/010, prepared for the Commodity Credit Corporation, U.S. Department of Agriculture, Washington, D.C., by Argonne National Laboratory, Argonne, Illinois, November.

Argonne, 2005b, Final Work Plan: Groundwater Monitoring at Morrill, Kansas, ANL/ER/TR05/003, prepared for the Commodity Credit Corporation, U.S. Department of Agriculture, Washington, D.C., by Argonne National Laboratory, Argonne, Illinois, August.

Argonne, 2006, September 2006 Monitoring Results for Morrill, Kansas, ANL/EVS/AGEM/CHRON-1016, prepared for the Commodity Credit Corporation, U.S. Department of Agriculture, Washington, D.C., by Argonne National Laboratory, Argonne, Illinois, December.

Argonne, 2007a, Final Report: Groundwater Monitoring at Morrill, Kansas, in September 2005 and March 2006, with Expansion of the Monitoring Network in January 2006, ANL/EVS/AGEM/TR-06-09, prepared for the Commodity Credit Corporation, U.S. Department of Agriculture, Washington, D.C., by Argonne National Laboratory, Argonne, Illinois, June. 
Argonne, 2007b, March-April 2007 Monitoring Results for Morrill, Kansas, ANL/EVS/AGEM/TR-07-06, prepared for the Commodity Credit Corporation, U.S. Department of Agriculture, by Argonne National Laboratory, Argonne, Illinois, May.

Argonne, 2008a, October 2007 Monitoring Results for Morrill, Kansas, ANL/EVS/AGEM/TR08-02, prepared for the Commodity Credit Corporation, U.S. Department of Agriculture, Washington, D.C., by Argonne National Laboratory, Argonne, Illinois, March.

Argonne, 2008b, April 2008 Monitoring Results for Morrill, Kansas, ANL/EVS/AGEM/TR-0815, prepared for the Commodity Credit Corporation, U.S. Department of Agriculture, Washington, D.C., by Argonne National Laboratory, Argonne, Illinois, July.

Argonne, 2009, October 2008 Monitoring Results for Morrill, Kansas, ANL/EVS/AGEM/TR-0903, prepared for the Commodity Credit Corporation, U.S. Department of Agriculture, Washington, D.C., by Argonne National Laboratory, Argonne, Illinois, March.

Argonne, 2010, Annual Report of Monitoring at Morrill, Kansas, in 2009, ANL/EVS/AGEM/TR10-04, prepared for the Commodity Credit Corporation, U.S. Department of Agriculture, Washington, D.C., by Argonne National Laboratory, Argonne, Illinois, August.

Argonne, 2011, Annual Report of Monitoring at Morrill, Kansas, in 2010, ANL/EVS/AGEM/TR11-06, prepared for the Commodity Credit Corporation, U.S. Department of Agriculture, Washington, D.C., by Argonne National Laboratory, Argonne, Illinois, June.

Argonne, 2012, Annual Report of Monitoring at Morrill, Kansas, in 2011, ANL/EVS/AGEM/TR12-05, prepared for the Commodity Credit Corporation, U.S. Department of Agriculture, Washington, D.C., by Argonne National Laboratory, Argonne, Illinois, March.

Argonne, 2013, Annual Report of Monitoring at Morrill, Kansas, in 2012, ANL/EVS/AGEM/TR13-03, prepared for the Commodity Credit Corporation, U.S. Department of Agriculture, Washington, D.C., by Argonne National Laboratory, Argonne, Illinois, May.

Argonne, 2014, Annual Report of Monitoring at Morrill, Kansas, in 2013, ANL/EVS/AGEM/TR13-09, prepared for the Commodity Credit Corporation, U.S. Department of Agriculture, Washington, D.C., by Argonne National Laboratory, Argonne, Illinois, March. 
Argonne, 2015, Annual Report of Monitoring at Morrill, Kansas, in 2014, ANL/EVS/AGEM/TR15-05, prepared for the Commodity Credit Corporation, U.S. Department of Agriculture, Washington, D.C., by Argonne National Laboratory, Argonne, Illinois, May.

Argonne, 2016, Annual Report of Monitoring at Morrill, Kansas, in 2015, ANL/EVS/AGEM/TR16-06, prepared for the Commodity Credit Corporation, U.S. Department of Agriculture, Washington, D.C., by Argonne National Laboratory, Argonne, Illinois, June.

Argonne, 2017, Annual Report of Monitoring at Morrill, Kansas, in 2016, ANL/EVS/AGEM/ TR17-06, prepared for the Commodity Credit Corporation, U.S. Department of Agriculture, Washington, D.C., by Argonne National Laboratory, Argonne, Illinois, May.

EPA, 1995, Method 524.2: Measurement of Purgeable Organic Compounds in Water by Capillary Column Gas Chromatography/Mass Spectrometry, Revision 4.1, edited by J.W. Munch, National Exposure Research Laboratory, Office of Research and Development, U.S. Environmental Protection Agency, Cincinnati, Ohio.

GeoCore, 1994a, Work Plan: Phase I Sampling and Site Visit: Brown County Groundwater Investigation, prepared for the Kansas Department of Health and Environment, Bureau of Environmental Remediation, Topeka, Kansas, by GeoCore Services, Inc., Salina, Kansas, April 12.

GeoCore, 1994b, Well Survey Material: Brown County Groundwater Investigation: Morrill PWS Well \#5 Site, field notes made by GeoCore Services, Inc., Salina, Kansas, during Phase I sampling of private wells, May 19.

GeoCore, 1994c, Work Plan: Phase II Drilling and Sampling: Brown County Groundwater Investigation, prepared for the Kansas Department of Health and Environment, Bureau of Environmental Remediation, Topeka, Kansas, by GeoCore Services, Inc., Salina, Kansas, July 14.

GeoCore, 1994d, Preliminary Report: Phase I Sampling: Brown County Groundwater Investigation, prepared for the Kansas Department of Health and Environment, Bureau of Environmental Remediation, Topeka, Kansas, by GeoCore Services, Inc., Salina, Kansas, July 19. 
GeoCore, 1996, Environmental Site Investigation Report: Phase II Drilling and Sampling: Morrill Public Water Supply Well \#5, Morrill, Kansas, prepared for the Kansas Department of Health and Environment, Bureau of Environmental Remediation, Topeka, Kansas, by GeoCore Services, Inc., Salina, Kansas, February 29.

KDHE, 1989, Preliminary Assessment of the Morrill Public Water Supply Well \#5, Morrill, Kansas (Site Identification Number: KS D981710288), Kansas Department of Health and Environment, Bureau of Environmental Remediation, Topeka, Kansas, June 6.

KDHE 2001, Missouri Basin Total Maximum Daily Load: Waterbody: Walnut Creek: Water Quality Impairment: Fecal Coliform Bacteria, Bureau of Water, Kansas Department of Health and Environment, Topeka, Kansas, August 28.

KDHE, 2005, letter from C. Carey (Bureau of Environmental Remediation, Kansas Department of Health and Environment, Topeka, Kansas) to C. Roe (Commodity Credit Corporation, U.S. Department of Agriculture, Washington, D.C.) regarding Final Work Plan: Groundwater Monitoring at Morrill, Kansas, October 31.

KDHE, 2007, letter from C. Carey (Bureau of Environmental Remediation, Kansas Department of Health and Environment, Topeka, Kansas) to C. Roe (Commodity Credit Corporation, U.S. Department of Agriculture, Washington, D.C.) regarding draft Morrill sampling report, January 5.

KDHE, 2008a, letter from C. Carey (Bureau of Environmental Remediation, Kansas Department of Health and Environment, Topeka, Kansas) to C. Roe (Commodity Credit Corporation, U.S. Department of Agriculture, Washington, D.C.) regarding revisions to the Morrill sampling report for September 2005 and March 2006, February 6.

KDHE, 2008b, letter from E. McWilliams (Bureau of Environmental Remediation, Kansas Department of Health and Environment, Topeka, Kansas) to C. Roe (Commodity Credit Corporation, U.S. Department of Agriculture, Washington, D.C.) regarding April 2008 Monitoring Report for Morrill, Kansas, August 6.

KDHE, 2010, Kansas Surface Water Register, Bureau of Environmental Field Services, Kansas Department of Health and Environment, Topeka, Kansas, December 15. 
KDHE, 2012, letter from H. Burke (Bureau of Environmental Remediation, Kansas Department of Health and Environment, Topeka, Kansas) to C. Roe (Commodity Credit Corporation, U.S. Department of Agriculture, Washington, D.C.) regarding Annual Report of Monitoring at Morrill, Kansas, in 2011, July 3.

KDHE, 2014, letter from H. Burke (Bureau of Environmental Remediation, Kansas Department of Health and Environment, Topeka, Kansas) to C. Roe (Commodity Credit Corporation, U.S. Department of Agriculture, Washington, D.C.) regarding Annual Report of Monitoring at Morrill, Kansas, in 2013, February 10.

KDHE, 2015, letter from D. Newman (Bureau of Environmental Remediation, Kansas Department of Health and Environment, Topeka, Kansas) to C. Roe (Commodity Credit Corporation, U.S. Department of Agriculture, Washington, D.C.) regarding Annual Report of Monitoring at Morrill, Kansas, in 2014, May 14.

KDHE, 2016a, letter from D. Newman (Bureau of Environmental Remediation, Kansas Department of Health and Environment, Topeka, Kansas) to C. Roe (Commodity Credit Corporation, U.S. Department of Agriculture, Washington, D.C.) regarding Annual Report of Monitoring at Morrill, Kansas, in 2015, May 16.

KDHE, 2016b, Risk Management Program Guidance, Policy and Scope of Work, BER-RS-057, Bureau of Environmental Remediation, Remedial Section, May 23.

KDHE, 2017, letter from K. Diediker (Bureau of Environmental Remediation, Department of Health and Environment, Topeka, Kansas) to C. Roe (Commodity Credit Corporation, U.S. Department of Agriculture, Washington, D.C.) regarding the Annual Report of Monitoring at Morrill, Kansas, in 2016, May 30.

Puls, R.W., and Barcelona, M.J., 1996, "Low-Flow (Minimal Drawdown) Ground-Water Sampling Procedures,” EPA/540/S-95/504, in Ground Water Issue, Superfund Technology Support Center for Ground Water, National Risk Management Research Laboratory, Ada, Oklahoma, April. 
Yeskis, D., and B. Zavala, 2002, Ground-Water Sampling Guidelines for Superfund and RCRA Project Managers: Ground Water Forum Issue Paper, EPA 542-S-02-001, Technology Innovative Office, Office of Solid Waste and Emergency Response, U.S. Environmental Protection Agency, Washington, D.C., May. 
Appendix A:

Sampling Activities at Morrill in 2017 
TABLE A.1 Sequence of sampling activities at Morrill, Kansas, in 2017.

\begin{tabular}{|c|c|c|c|c|c|c|c|c|}
\hline \multicolumn{2}{|c|}{ Sample } & Location & Sample & $\begin{array}{l}\text { Sampl } \\
\text { e } \\
\text { Type }^{\mathrm{a}}\end{array}$ & $\begin{array}{l}\text { Depth } \\
\text { (ft BGL) }\end{array}$ & $\begin{array}{l}\text { Sample } \\
\text { Matrix }\end{array}$ & $\begin{array}{c}\text { Shipment } \\
\text { Date }\end{array}$ & Sample Description \\
\hline $5 / 31 / 17$ & $17: 40$ & MW8S & MRMW8S-W-39797 & $\mathrm{N}$ & $10-25$ & WG & $6 / 1 / 2017$ & $\begin{array}{l}\text { Depth to water }=0.84 \mathrm{ft} \text { TOC. Depth of } 4 \text {-in. well }=26.8 \mathrm{ft} \text {. } \\
\text { Sample collected using low flow bladder pump after purging } \\
6 \mathrm{~L} \text {. Pump intake positioned at } 17.5 \mathrm{ft} \text {. }\end{array}$ \\
\hline $5 / 31 / 17$ & $18: 35$ & MW7S & MRMW7S-W-39796 & $\mathrm{N}$ & $20-45$ & WG & $6 / 1 / 2017$ & $\begin{array}{l}\text { Depth to water }=10.02 \mathrm{ft} \mathrm{TOC} \text {. Depth of } 4 \text {-in. well }=47 \mathrm{ft} \text {. } \\
\text { Sample collected using low flow bladder pump after purging } \\
6 \mathrm{~L} \text {. Pump intake positioned at } 32.5 \mathrm{ft} \text {. }\end{array}$ \\
\hline $5 / 31 / 17$ & 19:10 & MW9S & MRMW9S-W-39798 & $\mathrm{N}$ & $38.83-53.83$ & WG & $6 / 1 / 2017$ & $\begin{array}{l}\text { Depth to water }=18.65 \mathrm{ft} \text { TOC. Depth of } 2 \text {-in. well }=58.5 \mathrm{ft} \text {. } \\
\text { Sample collected using low flow bladder pump after purging } \\
5.5 \mathrm{~L} \text {. Pump intake positioned at } 46.33 \mathrm{ft} \text {. }\end{array}$ \\
\hline $6 / 1 / 17$ & $10: 00$ & QC & MRQCTB-W-39812 & TB & - & WQC & $6 / 1 / 2017$ & $\begin{array}{l}\text { Trip blank with water samples for VOC analysis shipped to } \\
\text { AGEM Laboratory. }\end{array}$ \\
\hline $6 / 1 / 17$ & $15: 00$ & MW5S & MRMW5S-W-39794 & $\mathrm{N}$ & $15-55$ & WG & $6 / 1 / 2017$ & $\begin{array}{l}\text { Depth to water }=12.02 \mathrm{ft} \text { TOC. Depth of } 4 \text {-in. well }=54.6 \mathrm{ft} \text {. } \\
\text { Sample collected using low flow bladder pump after purging } \\
6 \mathrm{~L} \text {. Pump intake positioned at } 35 \mathrm{ft} \text {. }\end{array}$ \\
\hline $6 / 1 / 17$ & $15: 00$ & MW5S & MRMW5S-W-39794VER & VER & $15-55$ & WG & $6 / 2 / 2017$ & Verification sample sent to Test America, Inc., Burlington, VT. \\
\hline $6 / 1 / 17$ & $15: 30$ & QC & MRQCIR-W-39808 & $\mathrm{RI}$ & - & WQC & $6 / 1 / 2017$ & $\begin{array}{l}\text { Rinsate of decontaminated sampling line after collection of } \\
\text { sample MRMW5S-W-39794. }\end{array}$ \\
\hline $6 / 1 / 17$ & $16: 20$ & MW3S & MRMW3S-W-39792 & $\mathrm{N}$ & $18-48$ & WG & $6 / 1 / 2017$ & $\begin{array}{l}\text { Depth to water }=17.79 \mathrm{ft} \text { TOC. Depth of } 4 \text {-in. well }=47.8 \mathrm{ft} \text {. } \\
\text { Sample collected using low flow bladder pump after purging } \\
6 \mathrm{~L} \text {. Pump intake positioned at } 33.04 \mathrm{ft} \text {. }\end{array}$ \\
\hline $6 / 1 / 17$ & $18: 18$ & MW1S & MRMW1S3X-W-39789 & N & $11-51$ & WG & $6 / 1 / 2017$ & $\begin{array}{l}\text { Depth to water }=17.55 \mathrm{ft} \mathrm{TOC} \text {. Depth of } 4 \text {-in. well }=54 \mathrm{ft} \text {. } \\
\text { Sample collected using Redi-Flow pump after purging } \\
72 \text { gal ( } 3 \text { well volume purge). Pump intake positioned at } \\
52 \mathrm{ft} .\end{array}$ \\
\hline $6 / 1 / 17$ & $18: 18$ & MW1S & MRMW1S3X-W-39789DUP & DUP-L & $11-51$ & WG & 6/1/2017 & Duplicate laboratory analysis. \\
\hline $6 / 1 / 17$ & $18: 30$ & $\mathrm{QC}$ & MRQCIR-W-39807 & $\mathrm{RI}$ & - & WQC & $6 / 1 / 2017$ & $\begin{array}{l}\text { Rinsate of decontaminated sampling line after collection of } \\
\text { sample MRMW1S3X-W-39789. }\end{array}$ \\
\hline $6 / 1 / 17$ & $18: 30$ & STONE & MRSTONE-W-39802 & $\mathrm{N}$ & 43 & WG & $6 / 1 / 2017$ & $\begin{array}{l}\text { Stone private well. Bail } 5 \mathrm{gal} \text {, then collect a sample using the } \\
\text { bailer. }\end{array}$ \\
\hline $6 / 1 / 17$ & $18: 55$ & $\begin{array}{l}\text { RILLINGE } \\
\quad \mathrm{R}\end{array}$ & MRRILLINGER-W-39801 & N & - & WG & $6 / 1 / 2017$ & $\begin{array}{l}\text { Rilinger private well. Let pump run for } 5 \text { min, then collect a } \\
\text { sample. }\end{array}$ \\
\hline $6 / 2 / 17$ & $10: 00$ & $\mathrm{QC}$ & MRDIH2O-W-39810 & FB & - & WQC & 6/2/2017 & Field blank of water used for equipment decontamination. \\
\hline $6 / 2 / 17$ & $10: 00$ & QC & MRQCTB-W-39813 & TB & - & WQC & $6 / 2 / 2017$ & $\begin{array}{l}\text { Trip blank with water samples for VOCs analyses shipped to } \\
\text { AGEM Laboratory. }\end{array}$ \\
\hline $6 / 2 / 17$ & $10: 00$ & $\mathrm{QC}$ & MRQCTB-W-39813VER & VER & - & WQC & 6/2/2017 & Verification sample sent to Test America, Inc., Burlington, VT. \\
\hline $6 / 2 / 17$ & $12: 00$ & MW11S & MRMW11SDUP-W-39804 & DUP-F & $53-68$ & WG & 6/2/2017 & Field replicate. \\
\hline $6 / 2 / 17$ & $12: 00$ & MW11S & MRMW11S-W-39800 & $\mathrm{N}$ & $53-68$ & WG & $6 / 2 / 2017$ & $\begin{array}{l}\text { Depth to water }=30.06 \mathrm{ft} \text { TOC. Depth of } 2 \text {-in. well }=72.7 \mathrm{ft} \text {. } \\
\text { Sample collected using low flow bladder pump after purging } \\
5.5 \mathrm{~L} \text {. Pump intake positioned at } 60.5 \mathrm{ft} \text {. }\end{array}$ \\
\hline $6 / 2 / 17$ & $12: 15$ & QC & MRQCIR-W-39809 & RI & - & WQC & $6 / 2 / 2017$ & $\begin{array}{l}\text { Rinsate of decontaminated sampling line after collection of } \\
\text { sample MRMW11S-W-39800. }\end{array}$ \\
\hline
\end{tabular}


TABLE A.1 (Cont.)

\begin{tabular}{|c|c|c|c|c|c|c|c|c|}
\hline \multicolumn{2}{|c|}{ Sample } & Location & Sample & $\begin{array}{l}\text { Sampl } \\
\text { e } \\
\text { Type }^{\mathrm{a}}\end{array}$ & $\begin{array}{l}\text { Depth } \\
\text { (ft BGL) }\end{array}$ & $\begin{array}{l}\text { Sample } \\
\text { Matrix }^{b}\end{array}$ & $\begin{array}{c}\text { Shipment } \\
\text { Date }\end{array}$ & Sample Description \\
\hline $6 / 2 / 17$ & $13: 00$ & MW6S & MRMW6S-W-39795 & $\mathrm{N}$ & $10-25$ & WG & 6/2/2017 & $\begin{array}{l}\text { Depth to water }=5.93 \mathrm{ft} \mathrm{TOC} \text {. Depth of } 4 \text {-in. well }=26.9 \mathrm{ft} \text {. } \\
\text { Sample collected using low flow bladder pump after purging }\end{array}$ \\
\hline $6 / 2 / 17$ & $14: 00$ & MW1D & MRMW1D-W-39790 & $\mathrm{N}$ & $63-88$ & WG & $6 / 2 / 2017$ & $\begin{array}{l}\text { Depth to water }=29.19 \mathrm{ft} \mathrm{TOC} \text {. Depth of } 4 \text {-in. well }=89 \mathrm{ft} \text {. } \\
\text { Sample collected using low flow bladder pump after purging } \\
6 \mathrm{~L} \text {. Pump intake positioned at } 75.5 \mathrm{ft} \text {. }\end{array}$ \\
\hline
\end{tabular}

a Sample types: DUP-F, field replicate; DUP-L, duplicate lab analysis; FB, field blank; N, primary sample; RI, rinsate; TB, trip blank; VER, verification sample; WASTE, investigation-derived waste sample.

b Matrix codes: SEDIMENT, surface water sediment; WG, groundwater; WQC, QA/QC water sample, e.g., trip blank; WS, surface water. 


\section{Appendix B:}

Results from the AGEM Laboratory and TestAmerica for Quality Control Samples Analyzed in 2017 
TABLE B.1 Analytical results from the AGEM Laboratory for quality control samples collected to monitor sample collection and handling activities in 2017.

\begin{tabular}{cccccc}
\hline & & & \multicolumn{2}{c}{ Concentration $(\mu \mathrm{g} / \mathrm{L})$} \\
\cline { 4 - 6 } $\begin{array}{c}\text { Sample } \\
\text { Date }\end{array}$ & Sample & $\begin{array}{c}\text { Sample } \\
\text { Type }\end{array}$ & $\begin{array}{c}\text { Carbon } \\
\text { Tetrachloride }\end{array}$ & $\begin{array}{c}\text { Methylene } \\
\text { Chloride }\end{array}$ \\
\hline $06 / 02 / 17$ & MRDIH2O-W-39810 & FB & ND & ND & ND \\
$06 / 01 / 17$ & MRQCIR-W-39808 & RI & ND & ND & ND \\
$06 / 01 / 17$ & MRQCIR-W-39807 & RI & ND & ND & ND \\
$06 / 02 / 17$ & MRQCIR-W-39809 & RI & ND & ND & ND \\
$06 / 01 / 17$ & MRQCTB-W-39812 & TB & ND & ND & ND \\
$06 / 02 / 17$ & MRQCTB-W-39813 & TB & ND & ND & ND \\
& & & & & \\
\hline
\end{tabular}

a Sample types: FB, field blank; RI, rinsate; TB, trip blank.

b ND, compound analyzed for but not detected at a level greater than or equal to the method detection limit $(<1 \mu \mathrm{g} / \mathrm{L})$. 
TABLE B.2 Analytical results from the AGEM Laboratory for dual analyses of samples collected in 2017.

\begin{tabular}{|c|c|c|c|c|c|c|c|c|c|}
\hline $\begin{array}{l}\text { Sample } \\
\text { Date }\end{array}$ & Location & Sample & $\begin{array}{c}\text { Sample } \\
\text { Type }^{a}\end{array}$ & $\begin{array}{c}\text { Depth } \\
\text { (ft (BGL) }\end{array}$ & $\begin{array}{l}\text { Sample } \\
\text { Matrix }^{b}\end{array}$ & $\begin{array}{c}\text { Carbon } \\
\text { Tetrachloride }\end{array}$ & Chloroform & $\begin{array}{c}\text { Methylene } \\
\text { Chloride }\end{array}$ & $\begin{array}{c}\text { Concentration } \\
\text { Units }\end{array}$ \\
\hline MW1S & MRMW1S3X-W-39789 & $6 / 1 / 17$ & $\mathrm{~N}$ & $11-51$ & WG & 22 & $0.7 \mathrm{Jc}$ & $N^{d}$ & $\mu \mathrm{g} / \mathrm{L}$ \\
\hline MW1S & MRMW1S3X-W-39789DUP & $6 / 1 / 17$ & DUP-L & $11-5$ & WG & 22 & 1.1 & ND & $\mu \mathrm{g} / \mathrm{L}$ \\
\hline MW11S & MRMW11S-W-39800 & $6 / 2 / 17$ & $\mathrm{~N}$ & $53-68$ & WG & 25 & ND & ND & $\mu \mathrm{g} / \mathrm{L}$ \\
\hline MW11S & MRMW11SDUP-W-39804 & $6 / 2 / 17$ & DUP-F & $53-68$ & WG & 23 & ND & ND & $\mu \mathrm{g} / \mathrm{L}$ \\
\hline
\end{tabular}

a Sample types: DUP-L, duplicate lab analysis; DUP-F, field replicate; N, primary sample.

b Matrix codes: WG, groundwater.

c J, compound identified with an estimated concentration between the instrument detection limit and the method detection limit.

d ND, compound analyzed for but not detected at a level greater than or equal to the method detection limit $(<1 \mu \mathrm{g} / \mathrm{L}$ for water). 
TABLE B.3 Results for groundwater samples collected in 2017 and submitted for verification organic analyses. ${ }^{\text {a }}$

\begin{tabular}{|c|c|c|c|c|c|c|c|c|}
\hline \multirow[b]{2}{*}{ Location } & \multirow[b]{2}{*}{ Sample } & \multirow[b]{2}{*}{$\begin{array}{l}\text { Sample } \\
\text { Date }\end{array}$} & \multirow[b]{2}{*}{$\begin{array}{l}\text { Depth } \\
\text { (ft BGL) }\end{array}$} & \multirow[b]{2}{*}{$\begin{array}{c}\text { Analytic } \\
\text { Laboratory }\end{array}$} & \multicolumn{4}{|c|}{ Concentration $(\mu \mathrm{g} / \mathrm{L})$} \\
\hline & & & & & $\begin{array}{c}\text { Carbon } \\
\text { Tetrachloride }\end{array}$ & Chloroform & $\begin{array}{l}\text { Methylene } \\
\text { Chloride }\end{array}$ & $\begin{array}{c}\text { Method Detection } \\
\text { Limit }\end{array}$ \\
\hline MW5S & MRMW1D-W-39794 & $6 / 1 / 17$ & $15-55$ & AGEM & $N D^{b}$ & ND & ND & 1 \\
\hline MW5S & MRMW1D-W-39794VER & $6 / 1 / 17$ & $15-55$ & TestAmerica & ND & ND & ND & 0.5 \\
\hline $\begin{array}{l}\mathrm{QC} \\
\mathrm{OC}\end{array}$ & $\begin{array}{l}\text { MRQCTB-W-39813 } \\
\text { MROCTB-W-39813VER }\end{array}$ & $6 / 2 / 17$ & - & $\begin{array}{c}\text { AGEM } \\
\text { TestAmerica }\end{array}$ & ND & ND & ND & 1 \\
\hline QC & MRQCTB-W-39813VER & $6 / 2 / 17$ & - & TestAmerica & ND & $0.2 \mathrm{Jc}^{\mathrm{c}}$ & ND & 0.5 \\
\hline
\end{tabular}

a TestAmerica verification data are in sample delivery group 200-38792 in Supplement 1 (on CD).

b ND, compound analyzed for but not detected at a level greater than or equal to the indicated method detection limit.

c J, compound identified with an estimated concentration between the instrument detection limit and the method detection limit. 
Supplement 1:

Sample Documentation from TestAmerica Laboratories, Inc., for Groundwater Verification Samples 


\section{TestAmerica}

THE LEADER IN ENVIRONMENTAL TESTING

\section{ANALYTICAL REPORT}

Job Number: 200-38792-1

SDG Number: 200-38792-1

Job Description: Morrill, KS (200-38792)

Contract Number: 6E-30541

For:

Argonne National Laboratory

9700 South Cass Avenue

Building 203

Office B-141

Argonne, IL 60439

Attention: Ms. Esther Bowen

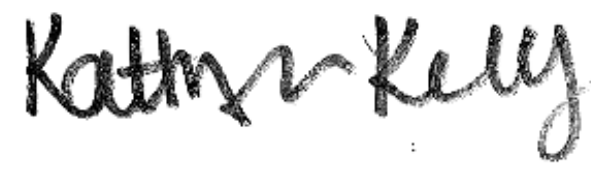

\section{Kathryn A Kelly, Project Manager I \\ 30 Community Drive, South Burlington, VT, 05403 \\ kathryn.kelly@testamericainc.com \\ 06/30/2017}

The test results in this report relate only to sample(s) as received by the laboratory. These test results were derived under a quality system that adheres to the requirements of NELAC. Pursuant to NELAC, this report may not be produced in full without written approval from the laboratory 


\section{Table of Contents}

Cover Title Page . . . . . . . . . . . . . . . . . 1

Data Summaries . . . . . . . . . . . . . . . . . . . 4

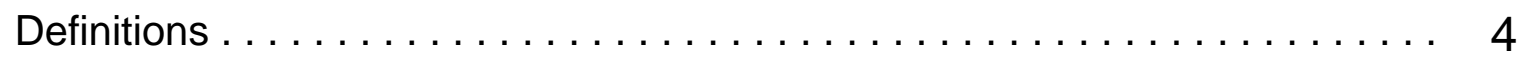

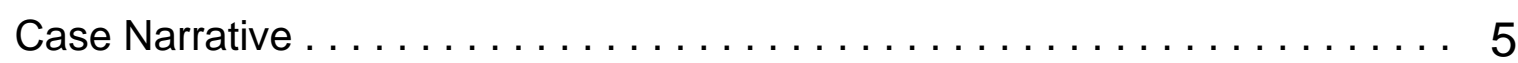

Detection Summary $\ldots \ldots \ldots \ldots \ldots \ldots \ldots \ldots \ldots \ldots \ldots \ldots \ldots \ldots \ldots \ldots$

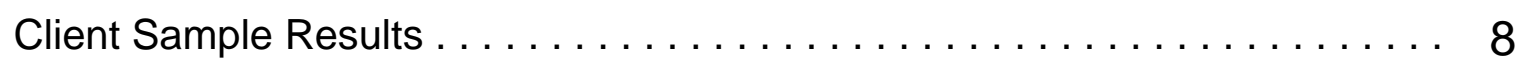

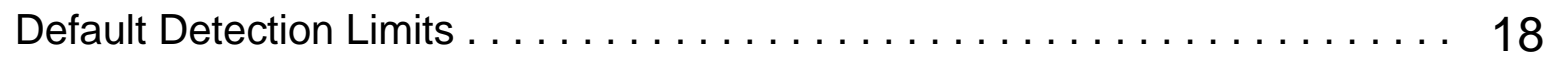

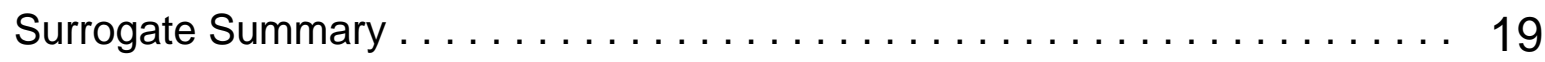

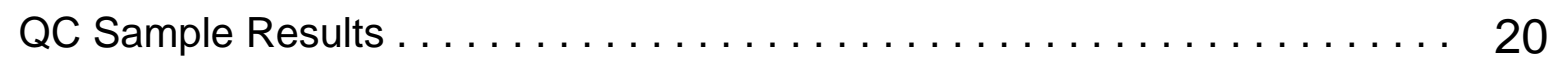

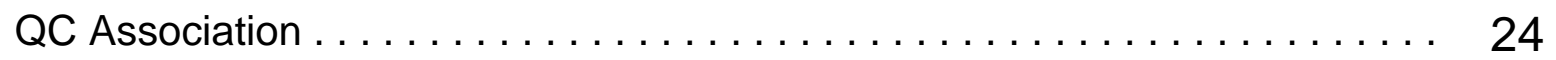

Chronicle ................................. 25

Certification Summary $\ldots \ldots \ldots \ldots \ldots \ldots \ldots \ldots \ldots \ldots \ldots \ldots \ldots \ldots \ldots \ldots \ldots$

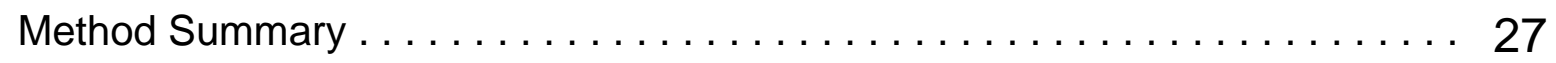

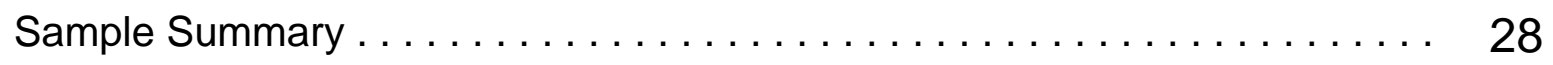

Manual Integration Summary . . . . . . . . . . . . . . . . 29

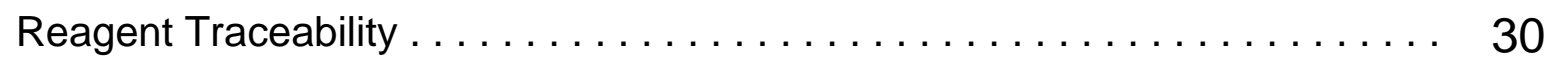

Organic Sample Data . . . . . . . . . . . . . . . . . . . . 37

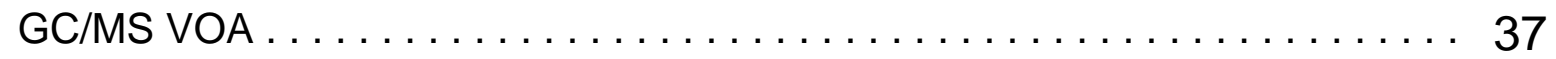

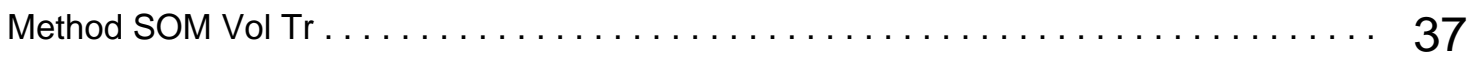

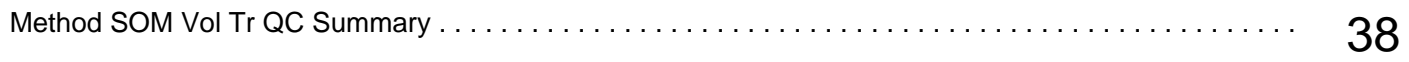

Method SOM Vol Tr Sample Data $\ldots \ldots \ldots \ldots \ldots \ldots \ldots \ldots \ldots \ldots \ldots \ldots \ldots \ldots \ldots \ldots, \quad 48$

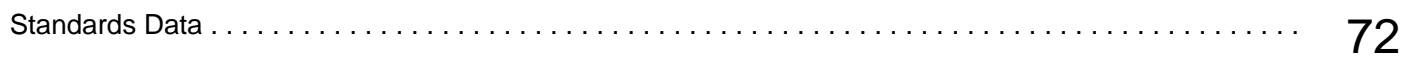

Method SOM Vol Tr ICAL Data $\ldots \ldots \ldots \ldots \ldots \ldots \ldots \ldots \ldots \ldots \ldots \ldots \ldots \ldots \ldots, 72$

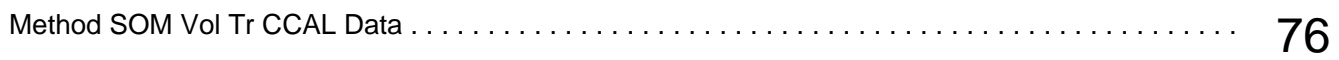

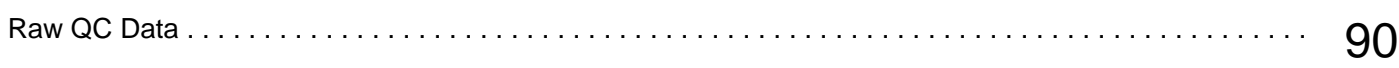

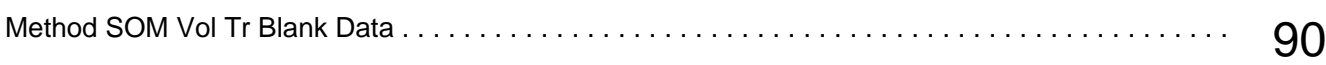




\section{Table of Contents}

Method SOM Vol Tr Run Logs $\ldots \ldots \ldots \ldots \ldots \ldots \ldots \ldots \ldots \ldots \ldots \ldots \ldots \ldots \ldots \ldots \ldots \ldots \ldots \ldots \ldots, 98$

Shipping and Receiving Documents $\ldots \ldots \ldots \ldots \ldots \ldots \ldots \ldots 101$

Client Chain of Custody ............................... 102

Sample Receipt Checklist ................................... 104 


\title{
Definitions/Glossary
}

\section{Qualifiers}

\section{GC/MS VOA}

Qualifier

Qualifier Description

$\overline{\mathrm{U}}$ Analyzed for but not detected.

B The analyte was found in an associated blank, as well as in the sample.

$\mathrm{J} \quad$ Indicates an estimated value.

* Surrogate is outside acceptance limits.

\section{GC/MS VOA TICs}

Qualifier

Qualifier Description

J Indicates an Estimated Value for TICs

$\mathrm{N} \quad$ This flag indicates the presumptive evidence of a compound.

B The analyte was found in an associated blank, as well as in the sample.

$X \quad$ See case narrative notes for explanation of the ' $X$ ' flag

\section{Glossary}

\author{
Abbreviation \\ These commonly used abbreviations may or may not be present in this report. \\ $\overline{\mathrm{a}}$ Listed under the "D" column to designate that the result is reported on a dry weight basis \\ $\% \mathrm{R} \quad$ Percent Recovery \\ CFL Contains Free Liquid \\ CNF Contains No Free Liquid \\ DER Duplicate Error Ratio (normalized absolute difference) \\ Dil Fac Dilution Factor \\ DL Detection Limit (DoD/DOE) \\ $\mathrm{DL}, \mathrm{RA}, \mathrm{RE}, \mathrm{IN}$ Indicates a Dilution, Re-analysis, Re-extraction, or additional Initial metals/anion analysis of the sample \\ DLC Decision Level Concentration (Radiochemistry) \\ EDL Estimated Detection Limit (Dioxin) \\ LOD Limit of Detection (DoD/DOE) \\ LOQ Limit of Quantitation (DoD/DOE) \\ MDA Minimum Detectable Activity (Radiochemistry) \\ MDC Minimum Detectable Concentration (Radiochemistry) \\ MDL Method Detection Limit \\ $\mathrm{ML} \quad$ Minimum Level (Dioxin) \\ NC Not Calculated \\ ND Not Detected at the reporting limit (or MDL or EDL if shown) \\ PQL Practical Quantitation Limit \\ QC Quality Control \\ RER Relative Error Ratio (Radiochemistry) \\ RL Reporting Limit or Requested Limit (Radiochemistry) \\ RPD Relative Percent Difference, a measure of the relative difference between two points \\ TEF Toxicity Equivalent Factor (Dioxin) \\ TEQ Toxicity Equivalent Quotient (Dioxin)
}




\title{
CASE NARRATIVE
}

\section{Client: Argonne National Laboratory}

\author{
Project: Morrill, KS (200-38792)
}

Report Number: 200-38792-1

With the exceptions noted as flags or footnotes, standard analytical protocols were followed in the analysis of the samples and no problems were encountered or anomalies observed. In addition all laboratory quality control samples were within established control limits, with any exceptions noted below. Each sample was analyzed to achieve the lowest possible reporting limit within the constraints of the method. In some cases, due to interference or analytes present at high concentrations, samples were diluted. For diluted samples, the reporting limits are adjusted relative to the dilution required.

Calculations are performed before rounding to avoid round-off errors in calculated results.

All holding times were met and proper preservation noted for the methods performed on these samples, unless otherwise detailed in the individual sections below.

\section{RECEIPT}

The samples were received on 06/03/2017; the samples arrived in good condition, properly preserved and on ice. The temperature of the coolers at receipt was $4.2 \mathrm{C}$.

\section{VOLATILE ORGANIC COMPOUNDS - TRACE}

Samples MRMW5S-W-39794, MRMW10S-W-39799, MRMW4S-W-39793, MRQCTB-W-39813 and VHBLK01 were analyzed for Volatile Organic Compounds - Trace in accordance with SOM02.3_Trace Volatiles. The samples were analyzed on 06/06/2017 and 06/08/2017.

Internal standard (ISTD) response for the following sample was outside of acceptance limits: MRQCTB-W-39813 (200-38792-4). The sample(s) was not re-analyzed due to insufficient volume. The internal standard 1,4-Dichlorobenzene-d4 fails marginally low due to carried over interference on the trap from previous samples. The data is presented as a secondary confirmation of results because the initial analysis contained an excess number of surrogate outages.

1,2,3-Trichlorobenzene, 1,2,4-Trichlorobenzene, 2-Butanone, Acetone and Carbon disulfide were detected in method blank MB $200-117296 / 7$ at levels that were above the method detection limit but below the reporting limit. The values should be considered estimates, and have been flagged. If the associated sample reported a result above the MDL and/or RL, the result has been flagged. Several analytes were detected in method blank MB 200-117409/6 at levels that were above the method detection limit but below the reporting limit. The values should be considered estimates, and have been flagged. If the associated sample reported a result above the MDL and/or RL, the result has been flagged. Refer to the QC report for details.

13 surrogates are used for this analysis. The laboratory's SOP allows 3 of these surrogates to be outside acceptance criteria without performing re-extraction/re-analysis. The following samples contained an allowable number of surrogate compounds outside limits: MRMW5S-W-39794 (200-38792-1), MRMW10S-W-39799 (200-38792-2), MRMW4S-W-39793 (200-38792-3) and VHBLK01

(200-38792-5). These results have been reported and qualified.

The continuing calibration verification (CCV) associated with batch 200-117296 recovered above the upper control limit for Styrene. The samples associated with this CCV were non-detects for the affected analytes; therefore, the data have been reported. The following samples are impacted: MRMW5S-W-39794 (200-38792-1), MRMW10S-W-39799 (200-38792-2), MRMW4S-W-39793 (200-38792-3), VHBLK01 (200-38792-5) and (CCVIS 200-117296/6).

The method allows two analytes and/or deuterated monitoring compounds (DMCs) to be outside of continuing calibration verification (CCV) technical acceptance criteria without performing re-analysis. The following sample is associated with a CCV which contains an allowable number of analytes outside \% Difference limits: MRQCTB-W-39813 (200-38792-4). The results have been reported.

A non-target constituent that represents a compound related to the DMC formulation is present in the following samples: MRMW5S-W-39794 (200-38792-1), MRMW10S-W-39799 (200-38792-2), MRMW4S-W-39793 (200-38792-3), MRQCTB-W-39813 (200-38792-4) and VHBLK01 (200-38792-5). The derived results for that compound have been qualified with an "X" qualifier to reflect the source of the contamination.

No additional analytical or quality issues were noted, other than those described above or in the Definitions/Glossary page. 


\begin{tabular}{|c|c|c|c|c|c|c|c|c|}
\hline Analyte & Result & Qualifier & RL & MDLE & Unit & Dil Fac D & Method & Prep Type \\
\hline Carbon disulfide & 0.15 & $\overline{J B}$ & 0.50 & & $\overline{\mathrm{ug} / \mathrm{L}}$ & $\overline{1}$ & $\begin{array}{l}\text { SOM02.2/NOA_ } \\
\mathrm{Tr}\end{array}$ & Total/NA \\
\hline 1,2,4-Trichlorobenzene & 0.068 & $J B$ & 0.50 & & $u g / L$ & 1 & $\begin{array}{l}\text { SOM02.2/NOA_ } \\
\text { Tr }\end{array}$ & Total/NA \\
\hline 1,2,3-Trichlorobenzene & 0.068 & $J B$ & 0.50 & & ug/L & 1 & $\begin{array}{l}\text { SOM02.2/NOA_ } \\
\text { Tr }\end{array}$ & Total/NA \\
\hline
\end{tabular}

Client Sample ID: MRMW10S-W-39799

Lab Sample ID: 200-38792-2

\begin{tabular}{|c|c|c|c|c|c|c|c|c|}
\hline Analyte & Result & Qualifier & $\mathbf{R L}$ & MDLE & Unit & Dil Fac D & Method & Prep Type \\
\hline Carbon disulfide & 0.11 & $\mathrm{JB}$ & 0.50 & & $\overline{u g / L}$ & $\overline{1}$ & $\begin{array}{l}\text { SOM02.2/VOA } \\
\mathrm{Tr}\end{array}$ & Total/NA \\
\hline Chloroform & 2.6 & & 0.50 & & ug/L & 1 & $\begin{array}{l}\text { SOM02.2/VOA_ } \\
\mathrm{Tr}\end{array}$ & Total/NA \\
\hline Carbon tetrachloride & 0.31 & $\mathrm{~J}$ & 0.50 & & ug/L & 1 & $\begin{array}{l}\text { SOM02.2/NOA_ } \\
\mathrm{Tr}\end{array}$ & Total/NA \\
\hline
\end{tabular}

Client Sample ID: MRMW4S-W-39793

Lab Sample ID: 200-38792-3

\begin{tabular}{|c|c|c|c|c|c|c|c|c|}
\hline Analyte & Result & Qualifier & $\mathbf{R L}$ & MDLE & Unit & Dil Fac $D$ & Method & Prep Type \\
\hline Carbon disulfide & 0.11 & $\overline{J B}$ & 0.50 & & $\overline{u g / L}$ & $\overline{1}$ & $\begin{array}{l}\text { SOM02.2/VOA_ } \\
\text { Tr }\end{array}$ & Total/NA \\
\hline Carbon tetrachloride & 0.11 & $\mathrm{~J}$ & 0.50 & & ug/L & 1 & $\begin{array}{l}\text { SOM02.2/NOA_ } \\
\text { Tr }\end{array}$ & Total/NA \\
\hline
\end{tabular}

Client Sample ID: MRQCTB-W-39813

Lab Sample ID: 200-38792-4

\begin{tabular}{|c|c|c|c|c|c|c|c|c|}
\hline Analyte & Result & Qualifier & $\mathbf{R L}$ & MDLE & Unit & Dil Fac D & Method & Prep Type \\
\hline Acetone & 5.1 & $\bar{B}$ & 5.0 & & $\overline{\mathrm{ug} / \mathrm{L}}$ & 1 & $\begin{array}{l}\text { SOM02.2/VOA_ } \\
\mathrm{Tr}\end{array}$ & Total/NA \\
\hline Acetone & 4.9 & $J B$ & 5.0 & & ug/L & 1 & $\begin{array}{l}\text { SOM02.2/NOA_ } \\
\text { Tr }\end{array}$ & Total/NA \\
\hline Carbon disulfide & 0.10 & $J B$ & 0.50 & & ug/L & 1 & $\begin{array}{l}\text { SOM02.2/NOA_ } \\
\text { Tr }\end{array}$ & Total/NA \\
\hline Carbon disulfide & 0.15 & $\mathrm{JB}$ & 0.50 & & ug/L & 1 & $\begin{array}{l}\text { SOM02.2/VOA } \\
\text { Tr }\end{array}$ & Total/NA \\
\hline Chloroform & 0.21 & $\mathrm{~J}$ & 0.50 & & $u g / L$ & 1 & $\begin{array}{l}\text { SOM02.2/NOA_ } \\
\mathrm{Tr}\end{array}$ & Total/NA \\
\hline Benzene & 0.23 & $\mathrm{~J}$ & 0.50 & & ug/L & 1 & $\begin{array}{l}\text { SOM02.2/VOA } \\
\mathrm{Tr}\end{array}$ & Total/NA \\
\hline Benzene & 0.23 & $\mathrm{~J}$ & 0.50 & & ug/L & 1 & $\begin{array}{l}\text { SOM02.2/VOA } \\
\mathrm{Tr}\end{array}$ & Total/NA \\
\hline Toluene & 1.2 & & 0.50 & & ug/L & 1 & $\begin{array}{l}\text { SOM02.2/VOA_ } \\
\text { Tr }\end{array}$ & Total/NA \\
\hline Toluene & 1.2 & & 0.50 & & $u g / L$ & 1 & $\begin{array}{l}\text { SOM02.2/NOA_ } \\
\mathrm{Tr}\end{array}$ & Total/NA \\
\hline Ethylbenzene & 0.079 & $\mathrm{~J}$ & 0.50 & & ug/L & 1 & $\begin{array}{l}\text { SOM02.2/VOA } \\
\text { Tr }\end{array}$ & Total/NA \\
\hline Ethylbenzene & 0.087 & $\mathrm{~J}$ & 0.50 & & ug/L & 1 & $\begin{array}{l}\text { SOM02.2/NOA_ } \\
\mathrm{Tr}\end{array}$ & Total/NA \\
\hline o-Xylene & 0.12 & $\mathrm{~J}$ & 0.50 & & ug/L & 1 & $\begin{array}{l}\text { SOM02.2/VOA } \\
\text { Tr }\end{array}$ & Total/NA \\
\hline o-Xylene & 0.12 & $\mathrm{~J}$ & 0.50 & & ug/L & 1 & $\begin{array}{l}\text { SOM02.2/VOA } \\
\mathrm{Tr}\end{array}$ & Total/NA \\
\hline $\mathrm{m}, \mathrm{p}$-Xylene & 0.31 & $\mathrm{~J}$ & 0.50 & & ug/L & 1 & $\begin{array}{l}\text { SOM02.2/NOA_ } \\
\mathrm{Tr}\end{array}$ & Total/NA \\
\hline
\end{tabular}

This Detection Summary does not include radiochemical test results. 
Client Sample ID: MRQCTB-W-39813 (Continued) Lab Sample ID: 200-38792-4

\begin{tabular}{|c|c|c|c|c|c|c|c|c|c|}
\hline Analyte & Result & Qualifier & RL & MDLE & Unit & Dil Fac & D & Method & Prep Type \\
\hline m,p-Xylene & 0.35 & $\bar{J}$ & 0.50 & & $\overline{u g} / \mathrm{L}$ & $\overline{1}$ & & $\begin{array}{l}\text { SOM02.2/VOA_ } \\
\mathrm{Tr}\end{array}$ & Total/NA \\
\hline
\end{tabular}

\section{Client Sample ID: VHBLK01}

Lab Sample ID: 200-38792-5

\begin{tabular}{|c|c|c|c|c|c|c|c|c|c|}
\hline Analyte & Result & Qualifier & $\mathbf{R L}$ & MDLE & Unit & Dil Fac & D & Method & Prep Type \\
\hline Acetone & 2.9 & $\overline{J B}$ & 5.0 & & $\overline{u g} / \mathrm{L}$ & $\overline{1}$ & & $\begin{array}{l}\text { SOM02.2/VOA_ } \\
\text { Tr }\end{array}$ & Total/NA \\
\hline Carbon disulfide & 0.10 & J B & 0.50 & & ug/L & 1 & & $\begin{array}{l}\text { SOM02.2/VOA_ } \\
\text { Tr }\end{array}$ & Total/NA \\
\hline
\end{tabular}




\section{Method: SOM02.2/VOA_Tr - Trace Water}

\section{Analyte}

Dichlorodifluoromethane

Chloromethane

Vinyl chloride

Bromomethane

Chloroethane

Trichlorofluoromethane

1,1-Dichloroethene

1,1,2-Trichloro-1,2,2-trifluoroethane

Acetone

Carbon disulfide

Methyl acetate

Methylene Chloride

trans-1,2-Dichloroethene

Methyl tert-butyl ether

1,1-Dichloroethane

cis-1,2-Dichloroethene

2-Butanone

Bromochloromethane

Chloroform

1,1,1-Trichloroethane

Cyclohexane

Carbon tetrachloride

Benzene

1,2-Dichloroethane

Trichloroethene

Methylcyclohexane

1,2-Dichloropropane

Bromodichloromethane

cis-1,3-Dichloropropene

4-Methyl-2-pentanone

Toluene

trans-1,3-Dichloropropene

1,1,2-Trichloroethane

Tetrachloroethene

2-Hexanone

Dibromochloromethane

1,2-Dibromoethane

Chlorobenzene

Ethylbenzene

o-Xylene

m,p-Xylene

Styrene

Bromoform

Isopropylbenzene

1,1,2,2-Tetrachloroethane

1,3-Dichlorobenzene

1,4-Dichlorobenzene

1,2-Dichlorobenzene

1,2-Dibromo-3-chloropropane
Result Qualifier

$0.50 \mathrm{U}$

$0.50 \mathrm{U}$

$0.50 \mathrm{U}$

$0.50 \mathrm{U}$

$0.50 \mathrm{U}$

$0.50 \mathrm{U}$

$0.50 \mathrm{U}$

$0.50 \mathrm{U}$

$5.0 \mathrm{U}$

$0.15 \mathrm{~J} \mathrm{~B}$

$0.50 \mathrm{U}$

$0.50 \mathrm{U}$

$0.50 \mathrm{U}$

$0.50 \mathrm{U}$

$0.50 \mathrm{U}$

$0.50 \mathrm{U}$

$5.0 \mathrm{U}$

$0.50 \mathrm{U}$

$0.50 \mathrm{U}$

$0.50 \mathrm{U}$

$0.50 \mathrm{U}$

$0.50 \mathrm{U}$

$0.50 \mathrm{U}$

$0.50 \mathrm{U}$

$0.50 \mathrm{U}$

$0.50 \mathrm{U}$

$0.50 \mathrm{U}$

$0.50 \mathrm{U}$

$0.50 \mathrm{U}$

$5.0 \mathrm{U}$

$0.50 \mathrm{U}$

$0.50 \mathrm{U}$

$0.50 \mathrm{U}$

$0.50 \mathrm{U}$

$5.0 \mathrm{U}$

$0.50 \mathrm{U}$

$0.50 \mathrm{U}$

$0.50 \mathrm{U}$

$0.50 \mathrm{U}$

$0.50 \mathrm{U}$

$0.50 \mathrm{U}$

$0.50 \mathrm{U}$

$0.50 \mathrm{U}$

$0.50 \mathrm{U}$

$0.50 \mathrm{U}$

$0.50 \mathrm{U}$

$0.50 \mathrm{U}$

$0.50 \mathrm{U}$

$0.50 \mathrm{U}$

\begin{tabular}{|c|c|c|c|c|}
\hline $\mathbf{R L}$ & MDLE & Unit & D & Prepared \\
\hline 0.50 & & $\overline{\mathrm{ug} / \mathrm{L}}$ & & \\
\hline 0.50 & & ug/L & & \\
\hline
\end{tabular}

ug/L

ug/L

ug/L

ug/L

ug/L

ug/L

ug/L

ug/L

ug/L

ug/L

ug/L

ug/L

ug/L

ug/L

ug/L

ug/L

ug/L

ug/L

$u g / L$

ug/L

ug/L

ug/L

ug/L

ug/L

ug/L

ug/L

ug/L

ug/L

ug/L

ug/L

ug/L

ug/L

ug/L

ug/L

ug/L

ug/L

ug/L

ug/L

ug/L

ug/L

ug/L

ug/L

ug/L

ug/L

ug/L

ug/L

ug/L

\begin{tabular}{|c|c|}
\hline Analyzed & \\
\hline 06/06/17 17:59 & \\
\hline 06/06/17 17:59 & \\
\hline 06/06/17 17:59 & \\
\hline 06/06/17 17:59 & \\
\hline 06/06/17 17:59 & \\
\hline 06/06/17 17:59 & \\
\hline 06/06/17 17:59 & \\
\hline 06/06/17 17:59 & \\
\hline 06/06/17 17:59 & \\
\hline 06/06/17 17:59 & \\
\hline 06/06/17 17:59 & \\
\hline 06/06/17 17:59 & \\
\hline 06/06/17 17:59 & \\
\hline 06/06/17 17:59 & \\
\hline 06/06/17 17:59 & \\
\hline 06/06/17 17:59 & \\
\hline 06/06/17 17:59 & \\
\hline 06/06/17 17:59 & \\
\hline 06/06/17 17:59 & \\
\hline 06/06/17 17:59 & \\
\hline 06/06/17 17:59 & \\
\hline 06/06/17 17:59 & \\
\hline 06/06/17 17:59 & \\
\hline 06/06/17 17:59 & \\
\hline 06/06/17 17:59 & \\
\hline 06/06/17 17:59 & \\
\hline 06/06/17 17:59 & \\
\hline 06/06/17 17:59 & \\
\hline 06/06/17 17:59 & \\
\hline 06/06/17 17:59 & \\
\hline 06/06/17 17:59 & \\
\hline 06/06/17 17:59 & \\
\hline 06/06/17 17:59 & \\
\hline $06 / 06 / 1717: 59$ & \\
\hline 06/06/17 17:59 & \\
\hline 06/06/17 17:59 & \\
\hline 06/06/17 17:59 & \\
\hline 06/06/17 17:59 & \\
\hline 06/06/17 17:59 & \\
\hline 06/06/17 17:59 & \\
\hline 06/06/17 17:59 & \\
\hline 06/06/17 17:59 & \\
\hline 06/06/17 17:59 & \\
\hline 06/06/17 17:59 & \\
\hline 06/06/17 17:59 & \\
\hline 06/06/17 17:59 & \\
\hline 06/06/17 17:59 & \\
\hline 06/06/17 17:59 & \\
\hline 06/06/17 17:59 & \\
\hline
\end{tabular}

TestAmerica Burlington 


\begin{tabular}{|c|c|c|c|c|c|c|c|c|c|}
\hline Analyte & Result & Qualifier & $\mathbf{R L}$ & & MDLE Uni & D & Prepared & Analyzed & Dil Fac \\
\hline 1,2,4-Trichlorobenzene & 0.068 & $\bar{J} \mathbf{B}$ & 0.50 & & $\overline{u g} / \mathrm{L}$ & & & $\overline{06 / 06 / 1717: 59}$ & $\overline{1}$ \\
\hline 1,2,3-Trichlorobenzene & 0.068 & J B & 0.50 & & ug/L & & & 06/06/17 17:59 & 1 \\
\hline Tentatively Identified Compound & Est. Result & Qualifier & Unit & $D$ & $R T$ & CAS No. & Prepared & Analyzed & Dil Fac \\
\hline Isoprene & 0.57 & $\overline{J N}$ & $\overline{u g / L}$ & & 2.83 & $78-79-5$ & & $\overline{06 / 06 / 17 ~ 17: 59}$ & $\overline{1}$ \\
\hline Unknown & 3.1 & $J B X$ & $u g / L$ & & 7.28 & & & 06/06/17 17:59 & 1 \\
\hline Cyclotrisiloxane, hexamethyl- & 0.54 & $J N$ & $u g / L$ & & 8.18 & $541-05-9$ & & 06/06/17 17:59 & 1 \\
\hline Cyclotetrasiloxane, octamethyl- & 1.9 & JN & $u g / L$ & & 11.01 & $556-67-2$ & & $06 / 06 / 1717: 59$ & 1 \\
\hline Total Alkanes & & & $u g / L$ & & & STL00989 & & 06/06/17 17:59 & 1 \\
\hline Surrogate & \%Recovery & Qualifier & Limits & & & & Prepared & Analyzed & Dil Fac \\
\hline Vinyl chloride-d3 & 106 & & $40-130$ & & & & & $\overline{06 / 06 / 17 ~ 17: 59}$ & $\overline{1}$ \\
\hline Chloroethane-d5 & 124 & & $65-130$ & & & & & 06/06/17 17:59 & 1 \\
\hline 1,1-Dichloroethene-d2 & 100 & & $60-125$ & & & & & 06/06/17 17:59 & 1 \\
\hline 2-Butanone-d5 & 150 & * & $40-130$ & & & & & $06 / 06 / 1717: 59$ & 1 \\
\hline Chloroform-d & 121 & & $70-125$ & & & & & 06/06/17 17:59 & 1 \\
\hline 1,2-Dichloroethane-d4 & 137 & * & $70-130$ & & & & & 06/06/17 17:59 & 1 \\
\hline Benzene-d6 & 118 & & $70-125$ & & & & & $06 / 06 / 1717: 59$ & 1 \\
\hline 1,2-Dichloropropane-d6 & 95 & & $60-140$ & & & & & 06/06/17 17:59 & 1 \\
\hline Toluene-d8 & 117 & & $70-130$ & & & & & 06/06/17 17:59 & 1 \\
\hline trans-1,3-Dichloropropene-d4 & 110 & & $55-130$ & & & & & $06 / 06 / 1717: 59$ & 1 \\
\hline 2-Hexanone-d5 & 145 & * & $45-130$ & & & & & 06/06/17 17:59 & 1 \\
\hline 1,1,2,2-Tetrachloroethane-d2 & 110 & & $65-120$ & & & & & 06/06/17 17:59 & 1 \\
\hline 1,2-Dichlorobenzene-d4 & 116 & & $80-120$ & & & & & $06 / 06 / 1717: 59$ & 1 \\
\hline
\end{tabular}

\section{Client Sample ID: MRMW10S-W-39799}

Lab Sample ID: 200-38792-2

Date Collected: 06/01/17 17:15

Matrix: Water

Date Received: 06/03/17 09:20

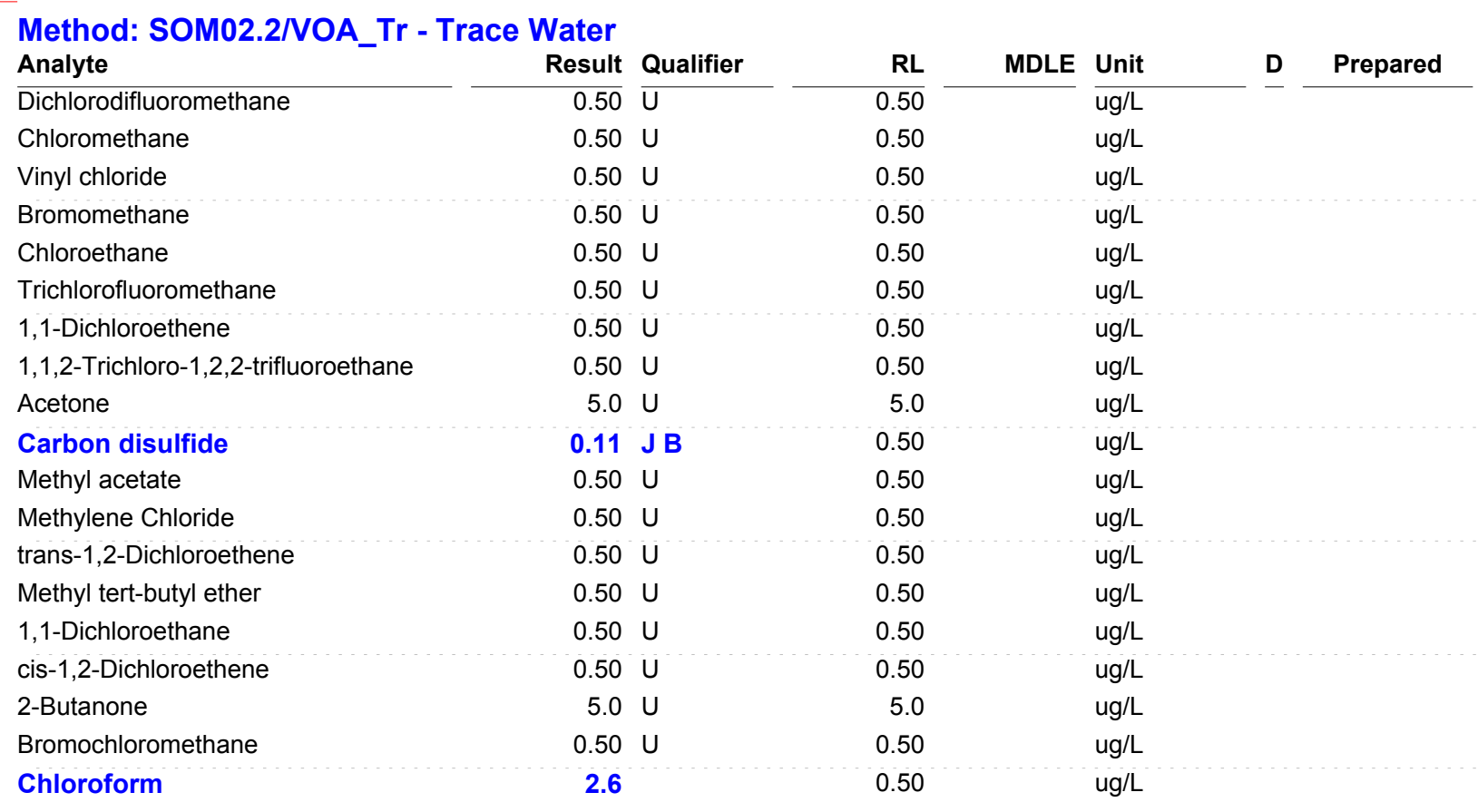

\begin{tabular}{|c|c|}
\hline Analyzed & Dil Fac \\
\hline$\overline{06 / 06 / 17 ~ 18: 25}$ & 1 \\
\hline 06/06/17 18:25 & \\
\hline 06/06/17 18:25 & \\
\hline $06 / 06 / 1718: 25$ & \\
\hline $06 / 06 / 17$ 18:25 & \\
\hline 06/06/17 18:25 & \\
\hline $06 / 06 / 1718: 25$ & \\
\hline 06/06/17 18:25 & \\
\hline 06/06/17 18:25 & \\
\hline $06 / 06 / 1718: 25$ & \\
\hline 06/06/17 18:25 & \\
\hline 06/06/17 18:25 & \\
\hline $06 / 06 / 1718: 25$ & \\
\hline 06/06/17 18:25 & \\
\hline 06/06/17 18:25 & \\
\hline $06 / 06 / 1718: 25$ & \\
\hline 06/06/17 18:25 & \\
\hline 06/06/17 18:25 & \\
\hline $06 / 06 / 1718: 25$ & \\
\hline
\end{tabular}




\begin{tabular}{|c|c|c|c|c|c|c|c|c|}
\hline Analyte & Result & Qualifier & $\mathbf{R L}$ & MDLE & Unit & D & Prepared & Analyzed \\
\hline 1,1,1-Trichloroethane & 0.50 & $\overline{\mathrm{U}}$ & 0.50 & & $\overline{u g} / \mathrm{L}$ & & & $\overline{06 / 06 / 17 ~ 18: 25}$ \\
\hline Cyclohexane & 0.50 & $U$ & 0.50 & & ug/L & & & 06/06/17 18:25 \\
\hline Carbon tetrachloride & 0.31 & $J$ & 0.50 & & $\mathrm{ug} / \mathrm{L}$ & & & 06/06/17 18:25 \\
\hline Benzene & 0.50 & $\mathrm{U}$ & 0.50 & & $u g / L$ & & & 06/06/17 18:25 \\
\hline 1,2-Dichloroethane & 0.50 & $\mathrm{U}$ & 0.50 & & ug/L & & & 06/06/17 18:25 \\
\hline Trichloroethene & 0.50 & $\mathrm{U}$ & 0.50 & & $\mathrm{ug} / \mathrm{L}$ & & & $06 / 06 / 1718: 25$ \\
\hline Methylcyclohexane & 0.50 & $U$ & 0.50 & & ug/L & & & 06/06/17 18:25 \\
\hline 1,2-Dichloropropane & 0.50 & $U$ & 0.50 & & ug/L & & & 06/06/17 18:25 \\
\hline Bromodichloromethane & 0.50 & $\mathrm{U}$ & 0.50 & & $\mathrm{ug} / \mathrm{L}$ & & & $06 / 06 / 1718: 25$ \\
\hline cis-1,3-Dichloropropene & 0.50 & $U$ & 0.50 & & ug/L & & & 06/06/17 18:25 \\
\hline 4-Methyl-2-pentanone & 5.0 & $U$ & 5.0 & & ug/L & & & 06/06/17 18:25 \\
\hline Toluene & 0.50 & $\mathrm{U}$ & 0.50 & & $\mathrm{ug} / \mathrm{L}$ & & & $06 / 06 / 1718: 25$ \\
\hline trans-1,3-Dichloropropene & 0.50 & $U$ & 0.50 & & ug/L & & & 06/06/17 18:25 \\
\hline 1,1,2-Trichloroethane & 0.50 & $U$ & 0.50 & & ug/L & & & 06/06/17 18:25 \\
\hline Tetrachloroethene & 0.50 & $\mathrm{U}$ & 0.50 & & $\mathrm{ug} / \mathrm{L}$ & & & $06 / 06 / 1718: 25$ \\
\hline 2-Hexanone & 5.0 & $U$ & 5.0 & & ug/L & & & 06/06/17 18:25 \\
\hline Dibromochloromethane & 0.50 & $U$ & 0.50 & & ug/L & & & 06/06/17 18:25 \\
\hline 1,2-Dibromoethane & 0.50 & $\mathrm{U}$ & 0.50 & & $\mathrm{ug} / \mathrm{L}$ & & & $06 / 06 / 1718: 25$ \\
\hline Chlorobenzene & 0.50 & $\mathrm{U}$ & 0.50 & & $u g / L$ & & & 06/06/17 18:25 \\
\hline Ethylbenzene & 0.50 & $U$ & 0.50 & & ug/L & & & 06/06/17 18:25 \\
\hline o-Xylene & 0.50 & $\mathrm{U}$ & 0.50 & & $\mathrm{ug} / \mathrm{L}$ & & & 06/06/17 18:25 \\
\hline m,p-Xylene & 0.50 & $U$ & 0.50 & & ug/L & & & 06/06/17 18:25 \\
\hline Styrene & 0.50 & $U$ & 0.50 & & ug/L & & & 06/06/17 18:25 \\
\hline Bromoform & 0.50 & $\mathrm{U}$ & 0.50 & & $\mathrm{ug} / \mathrm{L}$ & & & $06 / 06 / 1718: 25$ \\
\hline Isopropylbenzene & 0.50 & $U$ & 0.50 & & ug/L & & & 06/06/17 18:25 \\
\hline 1,1,2,2-Tetrachloroethane & 0.50 & $\mathrm{U}$ & 0.50 & & $u g / L$ & & & 06/06/17 18:25 \\
\hline 1,3-Dichlorobenzene & 0.50 & $\mathrm{U}$ & 0.50 & & $\mathrm{ug} / \mathrm{L}$ & & & $06 / 06 / 1718: 25$ \\
\hline 1,4-Dichlorobenzene & 0.50 & $U$ & 0.50 & & ug/L & & & 06/06/17 18:25 \\
\hline 1,2-Dichlorobenzene & 0.50 & $\mathrm{U}$ & 0.50 & & ug/L & & & 06/06/17 18:25 \\
\hline 1,2-Dibromo-3-chloropropane & 0.50 & $\mathrm{U}$ & 0.50 & & $\mathrm{ug} / \mathrm{L}$ & & & $06 / 06 / 1718: 25$ \\
\hline 1,2,4-Trichlorobenzene & 0.50 & $U$ & 0.50 & & ug/L & & & 06/06/17 18:25 \\
\hline 1,2,3-Trichlorobenzene & 0.50 & $U$ & 0.50 & & $u g / L$ & & & 06/06/17 18:25 \\
\hline
\end{tabular}

\begin{tabular}{|c|c|c|c|c|c|c|c|c|c|}
\hline Tentatively Identified Compound & Est. Result & Qualifier & Unit & $D$ & $R T$ & CAS No. & Prepared & Analyzed & Dil Fac \\
\hline Unknown & 3.0 & $\overline{J B X}$ & $\overline{u g / L}$ & & 7.28 & & & $\overline{06 / 06 / 17 ~ 18: 25}$ & 1 \\
\hline Total Alkanes & & & $u g / L$ & & & STL00989 & & 06/06/17 18:25 & \\
\hline Surrogate & \%Recovery & Qualifier & Limits & & & & Prepared & Analyzed & Dil Fac \\
\hline Vinyl chloride-d3 & 103 & & $40-130$ & & & & & $\overline{06 / 06 / 17 ~ 18: 25}$ & \\
\hline Chloroethane-d5 & 122 & & $65-130$ & & & & & 06/06/17 18:25 & \\
\hline 1,1-Dichloroethene-d2 & 96 & & $60-125$ & & & & & 06/06/17 18:25 & \\
\hline 2-Butanone-d5 & 149 & * & $40-130$ & & & & & $06 / 06 / 1718: 25$ & \\
\hline Chloroform-d & 124 & & $70-125$ & & & & & 06/06/17 18:25 & \\
\hline 1,2-Dichloroethane-d4 & 139 & * & $70-130$ & & & & & 06/06/17 18:25 & \\
\hline Benzene-d6 & 117 & & $70-125$ & & & & & $06 / 06 / 1718: 25$ & \\
\hline 1,2-Dichloropropane-d6 & 96 & & $60-140$ & & & & & 06/06/17 18:25 & \\
\hline Toluene-d8 & 117 & & $70-130$ & & & & & 06/06/17 18:25 & \\
\hline trans-1,3-Dichloropropene-d4 & 112 & & $55-130$ & & & & & $06 / 06 / 1718: 25$ & \\
\hline 2-Hexanone-d5 & 148 & * & $45-130$ & & & & & 06/06/17 18:25 & \\
\hline 1,1,2,2-Tetrachloroethane-d2 & 110 & & $65-120$ & & & & & $06 / 06 / 1718: 25$ & \\
\hline
\end{tabular}


Method: SOM02.2/VOA_Tr - Trace Water (Continued)

Surrogate
1,2 -Dichlorobenzene-d4 $\frac{\text { \%Recovery }}{119} \frac{\text { Qualifier }}{80-120} \frac{\text { Limits }}{80} \quad$ Prepared $\frac{\text { Analyzed }}{06 / 06 / 1718: 25} \frac{\text { Dil Fac }}{1}$

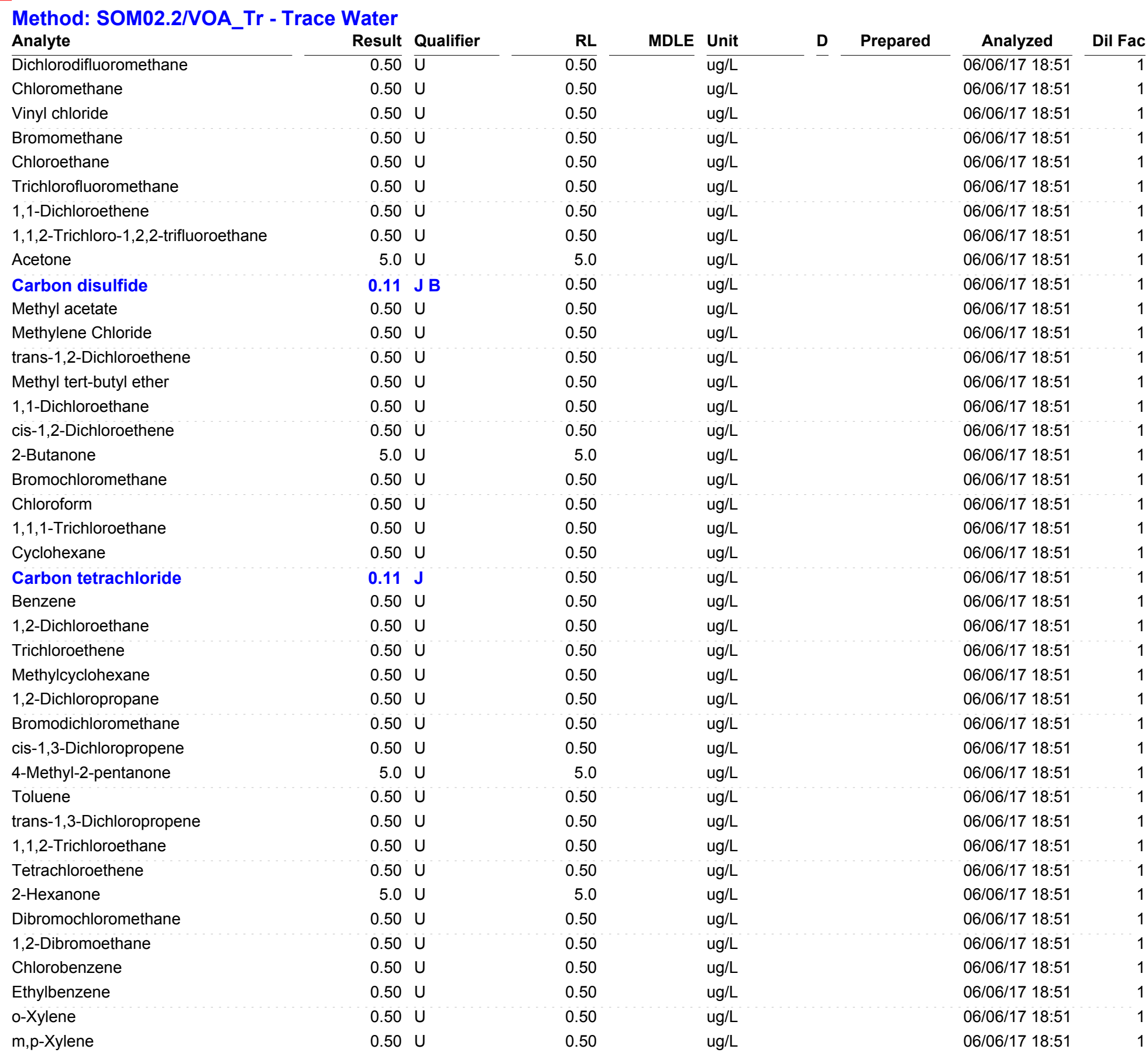




\begin{tabular}{|c|c|c|c|c|c|c|c|}
\hline Analyte & Result & Qualifier & RL & MDLE & Unit & D & Prepared \\
\hline Styrene & 0.50 & $\bar{U}$ & 0.50 & & $\overline{u g} / \mathrm{L}$ & & \\
\hline Bromoform & 0.50 & $\mathrm{U}$ & 0.50 & & $\mathrm{ug} / \mathrm{L}$ & & \\
\hline Isopropylbenzene & 0.50 & $\mathrm{U}$ & 0.50 & & $\mathrm{ug} / \mathrm{L}$ & & \\
\hline 1,1,2,2-Tetrachloroethane & 0.50 & $\mathrm{U}$ & 0.50 & & ug/L & & \\
\hline 1,3-Dichlorobenzene & 0.50 & $\mathrm{U}$ & 0.50 & & $\mathrm{ug} / \mathrm{L}$ & & \\
\hline 1,4-Dichlorobenzene & 0.50 & $U$ & 0.50 & & ug/L & & \\
\hline 1,2-Dichlorobenzene & 0.50 & $\mathrm{U}$ & 0.50 & & $\mathrm{ug} / \mathrm{L}$ & & \\
\hline 1,2-Dibromo-3-chloropropane & 0.50 & $\mathrm{U}$ & 0.50 & & $\mathrm{ug} / \mathrm{L}$ & & \\
\hline 1,2,4-Trichlorobenzene & 0.50 & $U$ & 0.50 & & $\mathrm{ug} / \mathrm{L}$ & & \\
\hline 1,2,3-Trichlorobenzene & 0.50 & $U$ & 0.50 & & $\mathrm{ug} / \mathrm{L}$ & & \\
\hline
\end{tabular}

Tentatively Identified Compound

Isoprene

Unknown

Total Alkanes

Unit

\section{Surrogate}

Vinyl chloride-d3

\%Recovery Qualifier

Chloroethane-d5

1,1-Dichloroethene-d2

2-Butanone-d5

Chloroform-d

1,2-Dichloroethane-d4

Benzene-d6

1,2-Dichloropropane-d6

Toluene-d8

trans-1,3-Dichloropropene-d4

2-Hexanone-d5

1,1,2,2-Tetrachloroethane-d2

1,2-Dichlorobenzene-d4
Est. Result Qualifier

\begin{tabular}{rll} 
Est. Result & Qualifier \\
\hline 0.51 & JN \\
3.0 & J BX
\end{tabular}

$3.0 J B X$

$u g / L$

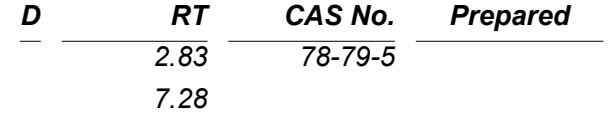

STL00989

\section{Prepared}

$65-130$

$60-125$

$40-130$

$70-125$

$70-130$

$70-125$

$60-140$

$70-130$

$55-130$

$45-130$

$65-120$

$80-120$

\begin{tabular}{|c|c|}
\hline Analyzed & Dil Fac \\
\hline$\overline{06 / 06 / 17 ~ 18: 51}$ & \\
\hline $06 / 06 / 17$ 18:51 & \\
\hline 06/06/17 18:51 & \\
\hline 06/06/17 18:51 & \\
\hline $06 / 06 / 17$ 18:51 & \\
\hline 06/06/17 18:51 & \\
\hline 06/06/17 18:51 & \\
\hline $06 / 06 / 1718: 51$ & \\
\hline 06/06/17 18:51 & \\
\hline 06/06/17 18:51 & \\
\hline
\end{tabular}

$\begin{array}{cr}\text { Analyzed } & \text { Dil Fac } \\ \text { 06/06/17 18:51 } & 1 \\ 06 / 06 / 17 \text { 18:51 } & 1 \\ 06 / 06 / 17 \text { 18:51 } & 1\end{array}$

\begin{tabular}{|c|c|}
\hline Analyzed & Dil Fa \\
\hline$\overline{06 / 06 / 17 ~ 18: 51}$ & \\
\hline 06/06/17 18:51 & \\
\hline 06/06/17 18:51 & \\
\hline $06 / 06 / 1718: 51$ & \\
\hline 06/06/17 18:51 & \\
\hline 06/06/17 18:51 & \\
\hline $06 / 06 / 1718: 51$ & \\
\hline 06/06/17 18:51 & \\
\hline 06/06/17 18:51 & \\
\hline 06/06/17 18:51 & \\
\hline 06/06/17 18:51 & \\
\hline 06/06/17 18:51 & \\
\hline $06 / 06 / 1718: 51$ & \\
\hline
\end{tabular}

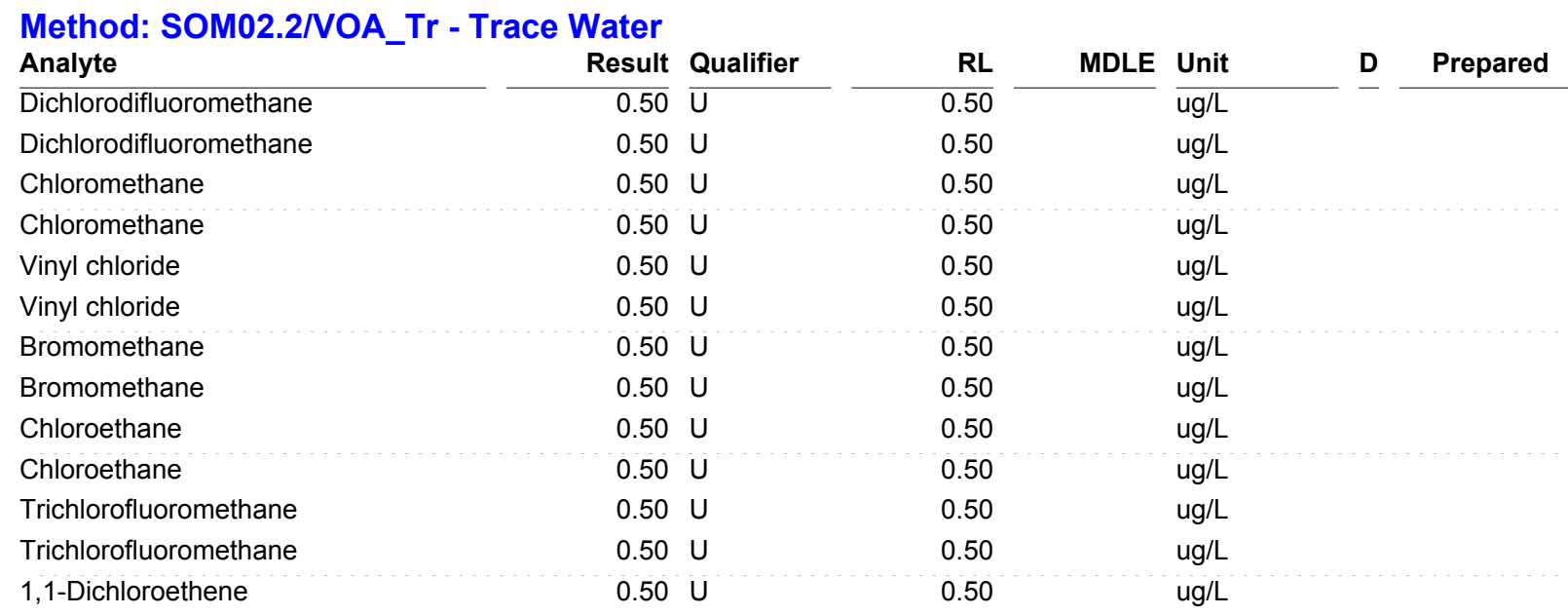

\begin{tabular}{|c|c|}
\hline Analyzed & Dil Fac \\
\hline$\overline{06 / 06 / 17 ~ 19: 17}$ & \\
\hline 06/08/17 22:50 & \\
\hline 06/06/17 19:17 & \\
\hline $06 / 08 / 1722: 50$ & \\
\hline 06/06/17 19:17 & \\
\hline $06 / 08 / 1722: 50$ & \\
\hline 06/06/17 19:17 & \\
\hline $06 / 08 / 1722: 50$ & \\
\hline 06/06/17 19:17 & \\
\hline $06 / 08 / 1722: 50$ & \\
\hline 06/06/17 19:17 & \\
\hline $06 / 08 / 1722: 50$ & \\
\hline $06 / 06 / 1719: 17$ & \\
\hline
\end{tabular}




\begin{tabular}{|c|c|c|c|c|c|c|c|c|c|}
\hline Analyte & Result & Qualifier & $\mathbf{R L}$ & MDLE & Unit & $\mathbf{D}$ & Prepared & Analyzed & Dil Fac \\
\hline 1,1-Dichloroethene & 0.50 & $\overline{\mathrm{U}}$ & 0.50 & & $\overline{u g} / \mathrm{L}$ & & & $\overline{06 / 08 / 1722: 50}$ & $\overline{1}$ \\
\hline 1,1,2-Trichloro-1,2,2-trifluoroethane & 0.50 & $\mathrm{U}$ & 0.50 & & $\mathrm{ug} / \mathrm{L}$ & & & 06/06/17 19:17 & 1 \\
\hline 1,1,2-Trichloro-1,2,2-trifluoroethane & 0.50 & $\mathrm{U}$ & 0.50 & & $\mathrm{ug} / \mathrm{L}$ & & & $06 / 08 / 1722: 50$ & 1 \\
\hline Acetone & 5.1 & B & 5.0 & & ug/L & & & 06/06/17 19:17 & 1 \\
\hline Acetone & 4.9 & J B & 5.0 & & ug/L & & & $06 / 08 / 1722: 50$ & 1 \\
\hline Carbon disulfide & 0.10 & J B & 0.50 & & $\mathrm{ug} / \mathrm{L}$ & & & 06/06/17 19:17 & 1 \\
\hline Carbon disulfide & 0.15 & J B & 0.50 & & ug/L & & & 06/08/17 22:50 & 1 \\
\hline Methyl acetate & 0.50 & $U$ & 0.50 & & ug/L & & & 06/06/17 19:17 & 1 \\
\hline Methyl acetate & 0.50 & $\mathrm{U}$ & 0.50 & & $\mathrm{ug} / \mathrm{L}$ & & & $06 / 08 / 1722: 50$ & 1 \\
\hline Methylene Chloride & 0.50 & $\mathrm{U}$ & 0.50 & & $\mathrm{ug} / \mathrm{L}$ & & & 06/06/17 19:17 & 1 \\
\hline Methylene Chloride & 0.50 & $\mathrm{U}$ & 0.50 & & ug/L & & & 06/08/17 22:50 & 1 \\
\hline trans-1,2-Dichloroethene & 0.50 & $\mathrm{U}$ & 0.50 & & $\mathrm{ug} / \mathrm{L}$ & & & 06/06/17 19:17 & 1 \\
\hline trans-1,2-Dichloroethene & 0.50 & $U$ & 0.50 & & ug/L & & & $06 / 08 / 1722: 50$ & 1 \\
\hline Methyl tert-butyl ether & 0.50 & $U$ & 0.50 & & ug/L & & & 06/06/17 19:17 & 1 \\
\hline Methyl tert-butyl ether & 0.50 & $\mathrm{U}$ & 0.50 & & $\mathrm{ug} / \mathrm{L}$ & & & $06 / 08 / 1722: 50$ & 1 \\
\hline 1,1-Dichloroethane & 0.50 & $\mathrm{U}$ & 0.50 & & ug/L & & & 06/06/17 19:17 & 1 \\
\hline 1,1-Dichloroethane & 0.50 & $\mathrm{U}$ & 0.50 & & ug/L & & & 06/08/17 22:50 & 1 \\
\hline cis-1,2-Dichloroethene & 0.50 & $\mathrm{U}$ & 0.50 & & $\mathrm{ug} / \mathrm{L}$ & & & 06/06/17 19:17 & 1 \\
\hline cis-1,2-Dichloroethene & 0.50 & $\mathrm{U}$ & 0.50 & & ug/L & & & 06/08/17 22:50 & 1 \\
\hline 2-Butanone & 5.0 & $\mathrm{U}$ & 5.0 & & ug/L & & & 06/06/17 19:17 & 1 \\
\hline 2-Butanone & 5.0 & $\mathrm{U}$ & 5.0 & & $\mathrm{ug} / \mathrm{L}$ & & & $06 / 08 / 1722: 50$ & 1 \\
\hline Bromochloromethane & 0.50 & $U$ & 0.50 & & ug/L & & & 06/06/17 19:17 & 1 \\
\hline Bromochloromethane & 0.50 & $U$ & 0.50 & & ug/L & & & 06/08/17 22:50 & 1 \\
\hline Chloroform & 0.21 & J & 0.50 & & $\mathrm{ug} / \mathrm{L}$ & & & 06/06/17 19:17 & 1 \\
\hline Chloroform & 0.50 & $U$ & 0.50 & & ug/L & & & 06/08/17 22:50 & 1 \\
\hline 1,1,1-Trichloroethane & 0.50 & $U$ & 0.50 & & ug/L & & & 06/06/17 19:17 & 1 \\
\hline 1,1,1-Trichloroethane & 0.50 & $\mathrm{U}$ & 0.50 & & $\mathrm{ug} / \mathrm{L}$ & & & $06 / 08 / 1722: 50$ & 1 \\
\hline Cyclohexane & 0.50 & $U$ & 0.50 & & ug/L & & & 06/06/17 19:17 & 1 \\
\hline Cyclohexane & 0.50 & $U$ & 0.50 & & ug/L & & & $06 / 08 / 1722: 50$ & 1 \\
\hline Carbon tetrachloride & 0.50 & $\mathrm{U}$ & 0.50 & & $\mathrm{ug} / \mathrm{L}$ & & & 06/06/17 19:17 & 1 \\
\hline Carbon tetrachloride & 0.50 & $U$ & 0.50 & & ug/L & & & 06/08/17 22:50 & 1 \\
\hline Benzene & 0.23 & $J$ & 0.50 & & ug/L & & & 06/06/17 19:17 & 1 \\
\hline Benzene & 0.23 & $\mathbf{J}$ & 0.50 & & $\mathrm{ug} / \mathrm{L}$ & & & $06 / 08 / 1722: 50$ & 1 \\
\hline 1,2-Dichloroethane & 0.50 & $U$ & 0.50 & & ug/L & & & 06/06/17 19:17 & 1 \\
\hline 1,2-Dichloroethane & 0.50 & $\mathrm{U}$ & 0.50 & & $\mathrm{ug} / \mathrm{L}$ & & & 06/08/17 22:50 & 1 \\
\hline Trichloroethene & 0.50 & $\mathrm{U}$ & 0.50 & & $\mathrm{ug} / \mathrm{L}$ & & & $06 / 06 / 1719: 17$ & 1 \\
\hline Trichloroethene & 0.50 & $U$ & 0.50 & & ug/L & & & 06/08/17 22:50 & 1 \\
\hline Methylcyclohexane & 0.50 & $U$ & 0.50 & & $u g / L$ & & & 06/06/17 19:17 & 1 \\
\hline Methylcyclohexane & 0.50 & $\mathrm{U}$ & 0.50 & & $\mathrm{ug} / \mathrm{L}$ & & & $06 / 08 / 1722: 50$ & 1 \\
\hline 1,2-Dichloropropane & 0.50 & $U$ & 0.50 & & ug/L & & & 06/06/17 19:17 & 1 \\
\hline 1,2-Dichloropropane & 0.50 & $U$ & 0.50 & & ug/L & & & 06/08/17 22:50 & 1 \\
\hline Bromodichloromethane & 0.50 & $\mathrm{U}$ & 0.50 & & $\mathrm{ug} / \mathrm{L}$ & & & 06/06/17 19:17 & 1 \\
\hline Bromodichloromethane & 0.50 & $\mathrm{U}$ & 0.50 & & ug/L & & & 06/08/17 22:50 & 1 \\
\hline cis-1,3-Dichloropropene & 0.50 & $\mathrm{U}$ & 0.50 & & ug/L & & & 06/06/17 19:17 & 1 \\
\hline cis-1,3-Dichloropropene & 0.50 & $\mathrm{U}$ & 0.50 & & $\mathrm{ug} / \mathrm{L}$ & & & $06 / 08 / 1722: 50$ & 1 \\
\hline 4-Methyl-2-pentanone & 5.0 & $U$ & 5.0 & & ug/L & & & 06/06/17 19:17 & 1 \\
\hline 4-Methyl-2-pentanone & 5.0 & $U$ & 5.0 & & ug/L & & & $06 / 08 / 1722: 50$ & 1 \\
\hline Toluene & 1.2 & & 0.50 & & $\mathrm{ug} / \mathrm{L}$ & & & 06/06/17 19:17 & 1 \\
\hline Toluene & 1.2 & & 0.50 & & ug/L & & & 06/08/17 22:50 & 1 \\
\hline
\end{tabular}




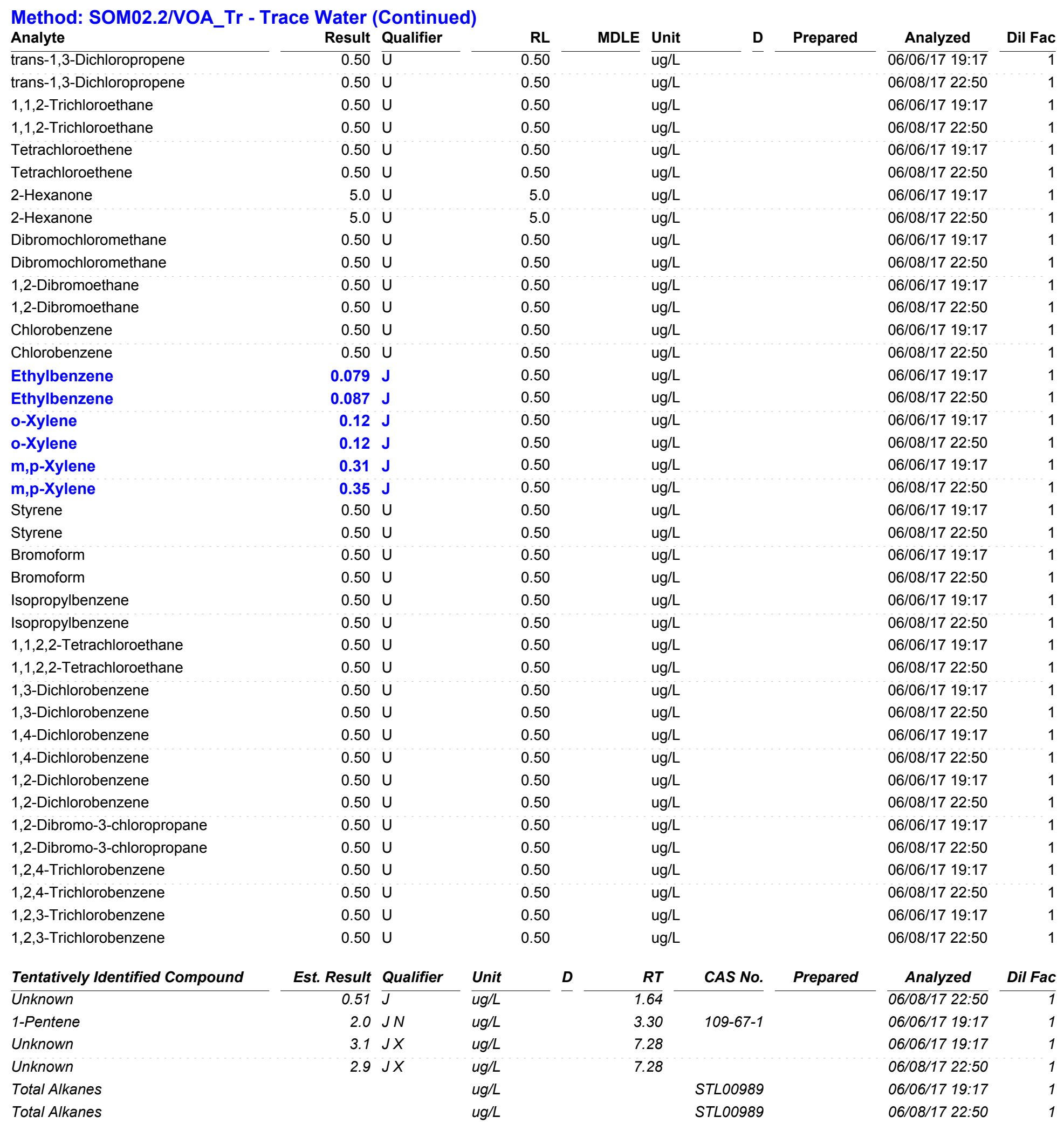




\begin{tabular}{|c|c|c|c|}
\hline Surrogate & $\%$ Recovery & Qualifier & Limits \\
\hline Vinyl chloride-d3 & 108 & & $40-130$ \\
\hline Vinyl chloride-d3 & 78 & & $40-130$ \\
\hline Chloroethane-d5 & 125 & & $65-130$ \\
\hline Chloroethane-d5 & 109 & & $65-130$ \\
\hline 1,1-Dichloroethene-d2 & 96 & & $60-125$ \\
\hline 1,1-Dichloroethene-d2 & 89 & & $60-125$ \\
\hline 2-Butanone-d5 & 155 & * & $40-130$ \\
\hline 2-Butanone-d5 & 124 & & $40-130$ \\
\hline Chloroform-d & 127 & * & $70-125$ \\
\hline Chloroform-d & 117 & & $70-125$ \\
\hline 1,2-Dichloroethane-d4 & 142 & * & $70-130$ \\
\hline 1,2-Dichloroethane-d4 & 137 & * & $70-130$ \\
\hline Benzene-d6 & 120 & & $70-125$ \\
\hline Benzene-d6 & 112 & & $70-125$ \\
\hline 1,2-Dichloropropane-d6 & 97 & & $60-140$ \\
\hline 1,2-Dichloropropane-d6 & 89 & & $60-140$ \\
\hline Toluene-d8 & 119 & & $70-130$ \\
\hline Toluene-d8 & 112 & & $70-130$ \\
\hline trans-1,3-Dichloropropene-d4 & 115 & & $55-130$ \\
\hline trans-1,3-Dichloropropene-d4 & 110 & & $55-130$ \\
\hline 2-Hexanone-d5 & 158 & * & $45-130$ \\
\hline 2-Hexanone-d5 & 132 & * & $45-130$ \\
\hline 1,1,2,2-Tetrachloroethane-d2 & 115 & & $65-120$ \\
\hline 1,1,2,2-Tetrachloroethane-d2 & 109 & & $65-120$ \\
\hline 1,2-Dichlorobenzene-d4 & 119 & & $80-120$ \\
\hline 1,2-Dichlorobenzene-d4 & 117 & & $80-120$ \\
\hline
\end{tabular}

\section{Client Sample ID: VHBLK01}

Date Collected: 06/03/17 11:08

Date Received: 06/03/17 09:20

\begin{tabular}{|c|c|c|}
\hline Prepared & Analyzed & Dil Fac \\
\hline & $\overline{06 / 06 / 17 ~ 19: 17}$ & 1 \\
\hline & $06 / 08 / 1722: 50$ & 1 \\
\hline & 06/06/17 19:17 & 1 \\
\hline & $06 / 08 / 1722: 50$ & 1 \\
\hline & 06/06/17 19:17 & 1 \\
\hline & $06 / 08 / 1722: 50$ & 1 \\
\hline & $06 / 06 / 1719: 17$ & 1 \\
\hline & $06 / 08 / 1722: 50$ & 1 \\
\hline & 06/06/17 19:17 & 1 \\
\hline & $06 / 08 / 1722: 50$ & 1 \\
\hline & 06/06/17 19:17 & 1 \\
\hline & $06 / 08 / 1722: 50$ & 1 \\
\hline & $06 / 06 / 1719: 17$ & 1 \\
\hline & $06 / 08 / 1722: 50$ & 1 \\
\hline & 06/06/17 19:17 & 1 \\
\hline & $06 / 08 / 1722: 50$ & 1 \\
\hline & 06/06/17 19:17 & 1 \\
\hline & $06 / 08 / 1722: 50$ & 1 \\
\hline & 06/06/17 19:17 & 1 \\
\hline & $06 / 08 / 1722: 50$ & 1 \\
\hline & 06/06/17 19:17 & 1 \\
\hline & $06 / 08 / 1722: 50$ & 1 \\
\hline & 06/06/17 19:17 & 1 \\
\hline & $06 / 08 / 1722: 50$ & 1 \\
\hline & $06 / 06 / 1719: 17$ & 1 \\
\hline & $06 / 08 / 1722: 50$ & 1 \\
\hline
\end{tabular}

Lab Sample ID: 200-38792-5 Matrix: Water

\begin{tabular}{|c|c|c|c|c|c|c|c|c|c|}
\hline . & Result & Qualifier & $\mathbf{R L}$ & MDLE & Unit & D & Prepared & Analyzed & Dil Fac \\
\hline Dichlorodifluoromethane & 0.50 & $\overline{\mathrm{U}}$ & 0.50 & & $\overline{u g} / \mathrm{L}$ & & & $\overline{06 / 06 / 17 ~ 19: 43}$ & 1 \\
\hline Chloromethane & 0.50 & $U$ & 0.50 & & ug/L & & & 06/06/17 19:43 & 1 \\
\hline Vinyl chloride & 0.50 & $U$ & 0.50 & & ug/L & & & 06/06/17 19:43 & 1 \\
\hline Bromomethane & 0.50 & $\mathrm{U}$ & 0.50 & & $\mathrm{ug} / \mathrm{L}$ & & & $06 / 06 / 1719: 43$ & 1 \\
\hline Chloroethane & 0.50 & $U$ & 0.50 & & ug/L & & & 06/06/17 19:43 & 1 \\
\hline Trichlorofluoromethane & 0.50 & $U$ & 0.50 & & ug/L & & & 06/06/17 19:43 & 1 \\
\hline 1,1-Dichloroethene & 0.50 & $U$ & 0.50 & & $\mathrm{ug} / \mathrm{L}$ & & & $06 / 06 / 1719: 43$ & 1 \\
\hline 1,1,2-Trichloro-1,2,2-trifluoroethane & 0.50 & $U$ & 0.50 & & ug/L & & & 06/06/17 19:43 & 1 \\
\hline Acetone & 2.9 & J B & 5.0 & & ug/L & & & 06/06/17 19:43 & 1 \\
\hline Carbon disulfide & 0.10 & J B & 0.50 & & $\mathrm{ug} / \mathrm{L}$ & & & 06/06/17 19:43 & 1 \\
\hline Methyl acetate & 0.50 & $U$ & 0.50 & & ug/L & & & 06/06/17 19:43 & 1 \\
\hline Methylene Chloride & 0.50 & $U$ & 0.50 & & ug/L & & & 06/06/17 19:43 & 1 \\
\hline trans-1,2-Dichloroethene & 0.50 & U & 0.50 & & $\mathrm{ug} / \mathrm{L}$ & & & 06/06/17 19:43 & 1 \\
\hline Methyl tert-butyl ether & 0.50 & $U$ & 0.50 & & ug/L & & & 06/06/17 19:43 & 1 \\
\hline 1,1-Dichloroethane & 0.50 & $U$ & 0.50 & & ug/L & & & 06/06/17 19:43 & 1 \\
\hline cis-1,2-Dichloroethene & 0.50 & $\mathrm{U}$ & 0.50 & & $\mathrm{ug} / \mathrm{L}$ & & & $06 / 06 / 1719: 43$ & 1 \\
\hline 2-Butanone & 5.0 & $U$ & 5.0 & & ug/L & & & 06/06/17 19:43 & 1 \\
\hline
\end{tabular}




\begin{tabular}{|c|c|c|c|c|}
\hline Analyte & Result & Qualifier & $\mathbf{R L}$ & MDLE \\
\hline Bromochloromethane & 0.50 & $\bar{U}$ & 0.50 & \\
\hline Chloroform & 0.50 & $\mathrm{U}$ & 0.50 & \\
\hline 1,1,1-Trichloroethane & 0.50 & $\mathrm{U}$ & 0.50 & \\
\hline Cyclohexane & 0.50 & $U$ & 0.50 & \\
\hline Carbon tetrachloride & 0.50 & $\mathrm{U}$ & 0.50 & \\
\hline Benzene & 0.50 & $U$ & 0.50 & \\
\hline 1,2-Dichloroethane & 0.50 & $U$ & 0.50 & \\
\hline Trichloroethene & 0.50 & $\mathrm{U}$ & 0.50 & \\
\hline Methylcyclohexane & 0.50 & $\mathrm{U}$ & 0.50 & \\
\hline 1,2-Dichloropropane & 0.50 & $\mathrm{U}$ & 0.50 & \\
\hline Bromodichloromethane & 0.50 & $\mathrm{U}$ & 0.50 & \\
\hline cis-1,3-Dichloropropene & 0.50 & $\mathrm{U}$ & 0.50 & \\
\hline 4-Methyl-2-pentanone & 5.0 & $\mathrm{U}$ & 5.0 & \\
\hline Toluene & 0.50 & $\mathrm{U}$ & 0.50 & \\
\hline trans-1,3-Dichloropropene & 0.50 & $U$ & 0.50 & \\
\hline 1,1,2-Trichloroethane & 0.50 & $U$ & 0.50 & \\
\hline Tetrachloroethene & 0.50 & $\mathrm{U}$ & 0.50 & \\
\hline 2-Hexanone & 5.0 & $\mathrm{U}$ & 5.0 & \\
\hline Dibromochloromethane & 0.50 & $U$ & 0.50 & \\
\hline 1,2-Dibromoethane & 0.50 & $\mathrm{U}$ & 0.50 & \\
\hline Chlorobenzene & 0.50 & $U$ & 0.50 & \\
\hline Ethylbenzene & 0.50 & $U$ & 0.50 & \\
\hline o-Xylene & 0.50 & $\mathrm{U}$ & 0.50 & \\
\hline m,p-Xylene & 0.50 & $U$ & 0.50 & \\
\hline Styrene & 0.50 & $U$ & 0.50 & \\
\hline Bromoform & 0.50 & $\mathrm{U}$ & 0.50 & \\
\hline Isopropylbenzene & 0.50 & $U$ & 0.50 & \\
\hline 1,1,2,2-Tetrachloroethane & 0.50 & $U$ & 0.50 & \\
\hline 1,3-Dichlorobenzene & 0.50 & $\mathrm{U}$ & 0.50 & \\
\hline 1,4-Dichlorobenzene & 0.50 & $\mathrm{U}$ & 0.50 & \\
\hline 1,2-Dichlorobenzene & 0.50 & $U$ & 0.50 & \\
\hline 1,2-Dibromo-3-chloropropane & 0.50 & $\mathrm{U}$ & 0.50 & \\
\hline 1,2,4-Trichlorobenzene & 0.50 & $U$ & 0.50 & \\
\hline 1,2,3-Trichlorobenzene & 0.50 & $U$ & 0.50 & \\
\hline
\end{tabular}

\begin{tabular}{|c|c|c|c|c|c|c|c|c|c|}
\hline Tentatively Identified Compound & Est. Result & Qualifier & Unit & $D$ & $R T$ & CAS No. & Prepared & Analyzed & Dil Fac \\
\hline Unknown & 2.9 & $J B X$ & $\overline{u g / L}$ & & 7.28 & & & $\overline{06 / 06 / 17 ~ 19: 43}$ & 1 \\
\hline Total Alkanes & & & $u g / L$ & & & STL00989 & & 06/06/17 19:43 & 1 \\
\hline Surrogate & $\%$ Recovery & Qualifier & Limits & & & & Prepared & Analyzed & Dil Fac \\
\hline Vinyl chloride-d3 & 102 & & $40-130$ & & & & & $\overline{06 / 06 / 17 ~ 19: 43}$ & $\overline{1}$ \\
\hline Chloroethane-d5 & 118 & & $65-130$ & & & & & 06/06/17 19:43 & 1 \\
\hline 1,1-Dichloroethene-d2 & 93 & & $60-125$ & & & & & 06/06/17 19:43 & 1 \\
\hline 2-Butanone-d5 & 123 & & $40-130$ & & & & & $06 / 06 / 1719: 43$ & 1 \\
\hline Chloroform-d & 122 & & $70-125$ & & & & & 06/06/17 19:43 & 1 \\
\hline 1,2-Dichloroethane-d4 & 137 & * & $70-130$ & & & & & 06/06/17 19:43 & 1 \\
\hline Benzene-d6 & 119 & & $70-125$ & & & & & $06 / 06 / 1719: 43$ & 1 \\
\hline 1,2-Dichloropropane-d6 & 96 & & $60-140$ & & & & & 06/06/17 19:43 & 1 \\
\hline Toluene-d8 & 116 & & $70-130$ & & & & & 06/06/17 19:43 & 1 \\
\hline trans-1,3-Dichloropropene-d4 & 108 & & $55-130$ & & & & & $06 / 06 / 1719: 43$ & \\
\hline
\end{tabular}




\section{Client Sample Results}

Client: Argonne National Laboratory

TestAmerica Job ID: 200-38792-1

Project/Site: Morrill, KS (200-38792)

SDG: 200-38792-1

Client Sample ID: VHBLK01

Lab Sample ID: 200-38792-5

Date Collected: 06/03/17 11:08

Matrix: Water

Date Received: 06/03/17 09:20

Method: SOM02.2/VOA_Tr - Trace Water (Continued)

\begin{tabular}{|c|c|c|c|}
\hline Surrogate & \%Recovery & Qualifier & Limits \\
\hline 2-Hexanone-d5 & $\overline{125}$ & & $45-130$ \\
\hline 1,1,2,2-Tetrachloroethane-d2 & 110 & & $65-120$ \\
\hline 1,2-Dichlorobenzene-d4 & 121 & * & $80-120$ \\
\hline
\end{tabular}

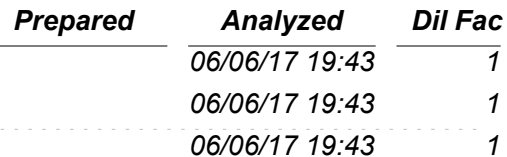

TestAmerica Burlington 


\section{Default Detection Limits}

\section{Method: SOM02.2/VOA_Tr - Trace Water}

\begin{tabular}{|c|c|c|}
\hline Analyte & $\mathbf{R L}$ & MDLE \\
\hline 1,1,1-Trichloroethane & 0.50 & 0.10 \\
\hline 1,1,2,2-Tetrachloroethane & 0.50 & 0.079 \\
\hline 1,1,2-Trichloro-1,2,2-trifluoroethane & 0.50 & 0.12 \\
\hline 1,1,2-Trichloroethane & 0.50 & 0.085 \\
\hline 1,1-Dichloroethane & 0.50 & 0.10 \\
\hline 1,1-Dichloroethene & 0.50 & 0.090 \\
\hline 1,2,3-Trichlorobenzene & 0.50 & 0.059 \\
\hline 1,2,4-Trichlorobenzene & 0.50 & 0.068 \\
\hline 1,2-Dibromo-3-chloropropane & 0.50 & 0.13 \\
\hline 1,2-Dibromoethane & 0.50 & 0.10 \\
\hline 1,2-Dichlorobenzene & 0.50 & 0.075 \\
\hline 1,2-Dichloroethane & 0.50 & 0.092 \\
\hline 1,2-Dichloropropane & 0.50 & 0.19 \\
\hline 1,3-Dichlorobenzene & 0.50 & 0.069 \\
\hline 1,4-Dichlorobenzene & 0.50 & 0.078 \\
\hline 2-Butanone & 5.0 & 1.0 \\
\hline 2-Hexanone & 5.0 & 0.64 \\
\hline 4-Methyl-2-pentanone & 5.0 & 0.98 \\
\hline Acetone & 5.0 & 1.3 \\
\hline Benzene & 0.50 & 0.17 \\
\hline Bromochloromethane & 0.50 & 0.10 \\
\hline Bromodichloromethane & 0.50 & 0.072 \\
\hline Bromoform & 0.50 & 0.091 \\
\hline Bromomethane & 0.50 & 0.097 \\
\hline Carbon disulfide & 0.50 & 0.091 \\
\hline Carbon tetrachloride & 0.50 & 0.088 \\
\hline Chlorobenzene & 0.50 & 0.085 \\
\hline Chloroethane & 0.50 & 0.17 \\
\hline Chloroform & 0.50 & 0.10 \\
\hline Chloromethane & 0.50 & 0.10 \\
\hline cis-1,2-Dichloroethene & 0.50 & 0.094 \\
\hline cis-1,3-Dichloropropene & 0.50 & 0.12 \\
\hline Cyclohexane & 0.50 & 0.11 \\
\hline Dibromochloromethane & 0.50 & 0.079 \\
\hline Dichlorodifluoromethane & 0.50 & 0.096 \\
\hline Ethylbenzene & 0.50 & 0.058 \\
\hline Isopropylbenzene & 0.50 & 0.040 \\
\hline m,p-Xylene & 0.50 & 0.058 \\
\hline Methyl acetate & 0.50 & 0.12 \\
\hline Methyl tert-butyl ether & 0.50 & 0.080 \\
\hline Methylcyclohexane & 0.50 & 0.19 \\
\hline Methylene Chloride & 0.50 & 0.098 \\
\hline o-Xylene & 0.50 & 0.040 \\
\hline Styrene & 0.50 & 0.041 \\
\hline Tetrachloroethene & 0.50 & 0.070 \\
\hline Toluene & 0.50 & 0.14 \\
\hline trans-1,2-Dichloroethene & 0.50 & 0.11 \\
\hline trans-1,3-Dichloropropene & 0.50 & 0.034 \\
\hline Trichloroethene & 0.50 & 0.18 \\
\hline Trichlorofluoromethane & 0.50 & 0.12 \\
\hline Vinyl chloride & 0.50 & 0.097 \\
\hline
\end{tabular}

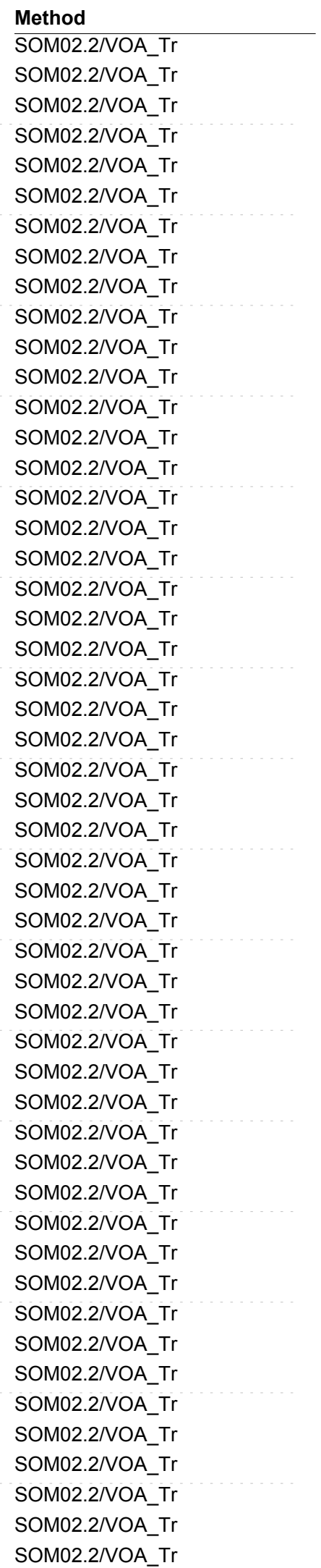




\section{Method: SOM02.2/VOA_Tr - Trace Water}

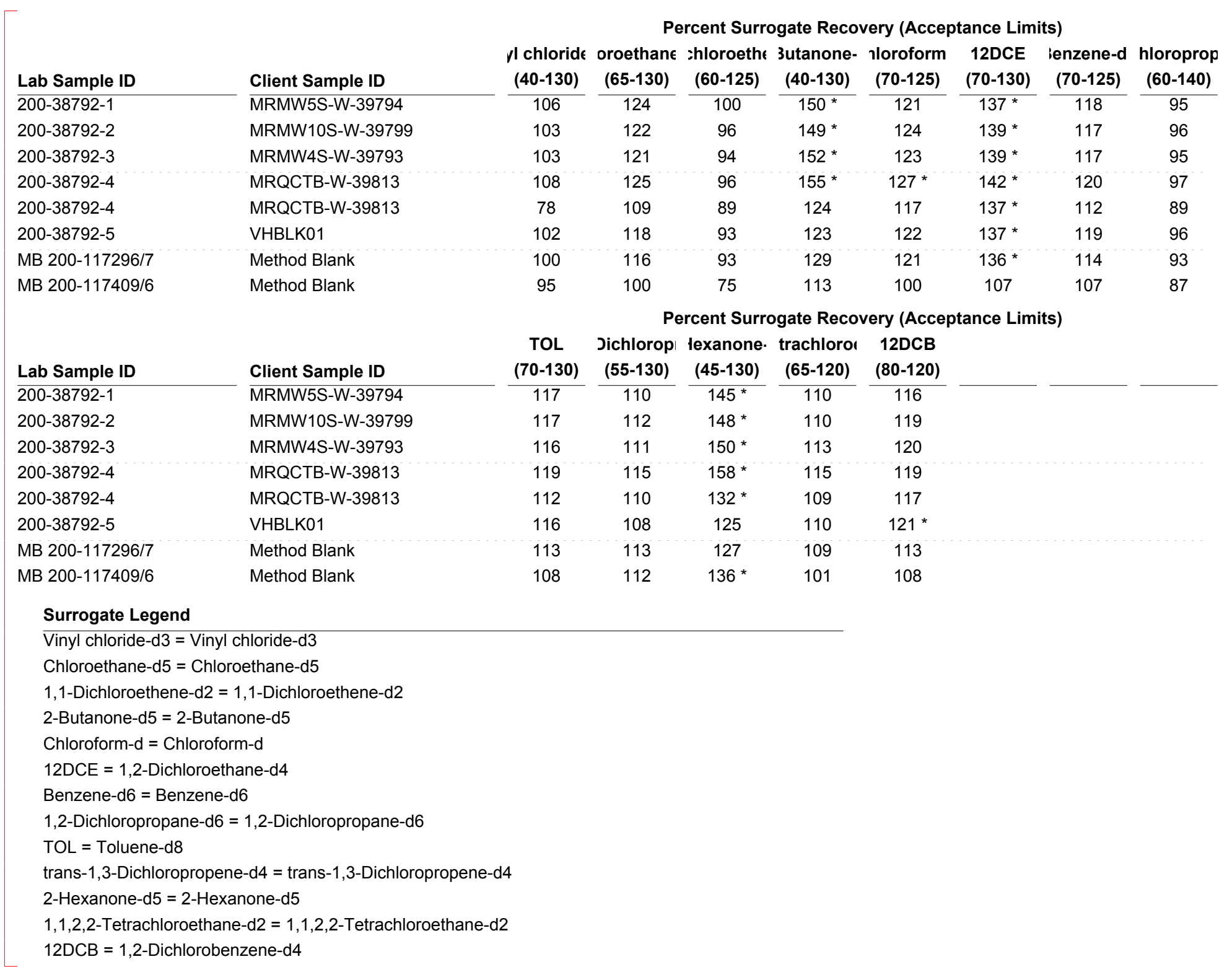


Lab Sample ID: MB 200-117296/7

Matrix: Water

Analysis Batch: 117296

\begin{tabular}{|c|c|c|c|c|c|c|c|c|}
\hline Analyte & $\begin{array}{r}\text { MB } \\
\text { Result }\end{array}$ & $\begin{array}{l}\text { MB } \\
\text { Qualifier }\end{array}$ & $\mathbf{R L}$ & MDLE & Unit & Prepared & Analyzed & Dil Fac \\
\hline Dichlorodifluoromethane & 0.50 & $\overline{\mathrm{U}}$ & 0.50 & & $\overline{u g} / \mathrm{L}$ & & $\overline{06 / 06 / 1715: 43}$ & $\overline{1}$ \\
\hline Chloromethane & 0.50 & $U$ & 0.50 & & ug/L & & 06/06/17 15:43 & 1 \\
\hline Vinyl chloride & 0.50 & $U$ & 0.50 & & ug/L & & 06/06/17 15:43 & 1 \\
\hline Bromomethane & 0.50 & $\mathrm{U}$ & 0.50 & & $\mathrm{ug} / \mathrm{L}$ & & $06 / 06 / 1715: 43$ & 1 \\
\hline Chloroethane & 0.50 & $U$ & 0.50 & & ug/L & & 06/06/17 15:43 & 1 \\
\hline Trichlorofluoromethane & 0.50 & $U$ & 0.50 & & ug/L & & 06/06/17 15:43 & 1 \\
\hline 1,1-Dichloroethene & 0.50 & $\mathrm{U}$ & 0.50 & & $\mathrm{ug} / \mathrm{L}$ & & $06 / 06 / 1715: 43$ & 1 \\
\hline 1,1,2-Trichloro-1,2,2-trifluoroethane & 0.50 & $U$ & 0.50 & & ug/L & & 06/06/17 15:43 & 1 \\
\hline Acetone & 4.8 & $\mathrm{~J}$ & 5.0 & & ug/L & & 06/06/17 15:43 & 1 \\
\hline Carbon disulfide & 0.14 & $\mathrm{~J}$ & 0.50 & & $\mathrm{ug} / \mathrm{L}$ & & $06 / 06 / 1715: 43$ & 1 \\
\hline Methyl acetate & 0.50 & $U$ & 0.50 & & ug/L & & 06/06/17 15:43 & 1 \\
\hline Methylene Chloride & 0.50 & $U$ & 0.50 & & ug/L & & 06/06/17 15:43 & 1 \\
\hline trans-1,2-Dichloroethene & 0.50 & $\mathrm{U}$ & 0.50 & & $\mathrm{ug} / \mathrm{L}$ & & $06 / 06 / 1715: 43$ & 1 \\
\hline Methyl tert-butyl ether & 0.50 & $U$ & 0.50 & & ug/L & & 06/06/17 15:43 & 1 \\
\hline 1,1-Dichloroethane & 0.50 & $U$ & 0.50 & & ug/L & & 06/06/17 15:43 & 1 \\
\hline cis-1,2-Dichloroethene & 0.50 & $\mathrm{U}$ & 0.50 & & $\mathrm{ug} / \mathrm{L}$ & & $06 / 06 / 1715: 43$ & 1 \\
\hline 2-Butanone & 1.0 & $J$ & 5.0 & & ug/L & & 06/06/17 15:43 & 1 \\
\hline Bromochloromethane & 0.50 & $U$ & 0.50 & & ug/L & & 06/06/17 15:43 & 1 \\
\hline Chloroform & 0.50 & $\mathrm{U}$ & 0.50 & & $\mathrm{ug} / \mathrm{L}$ & & $06 / 06 / 1715: 43$ & 1 \\
\hline 1,1,1-Trichloroethane & 0.50 & $U$ & 0.50 & & ug/L & & 06/06/17 15:43 & 1 \\
\hline Cyclohexane & 0.50 & $U$ & 0.50 & & ug/L & & 06/06/17 15:43 & 1 \\
\hline Carbon tetrachloride & 0.50 & $U$ & 0.50 & & $\mathrm{ug} / \mathrm{L}$ & & $06 / 06 / 1715: 43$ & 1 \\
\hline Benzene & 0.50 & $U$ & 0.50 & & ug/L & & 06/06/17 15:43 & 1 \\
\hline 1,2-Dichloroethane & 0.50 & $U$ & 0.50 & & ug/L & & 06/06/17 15:43 & 1 \\
\hline Trichloroethene & 0.50 & U & 0.50 & & $\mathrm{ug} / \mathrm{L}$ & & $06 / 06 / 1715: 43$ & 1 \\
\hline Methylcyclohexane & 0.50 & $U$ & 0.50 & & ug/L & & 06/06/17 15:43 & 1 \\
\hline 1,2-Dichloropropane & 0.50 & $U$ & 0.50 & & ug/L & & 06/06/17 15:43 & 1 \\
\hline Bromodichloromethane & 0.50 & $\mathrm{U}$ & 0.50 & & $\mathrm{ug} / \mathrm{L}$ & & $06 / 06 / 1715: 43$ & 1 \\
\hline cis-1,3-Dichloropropene & 0.50 & $U$ & 0.50 & & ug/L & & 06/06/17 15:43 & 1 \\
\hline 4-Methyl-2-pentanone & 5.0 & $U$ & 5.0 & & ug/L & & 06/06/17 15:43 & 1 \\
\hline Toluene & 0.50 & $U$ & 0.50 & & $\mathrm{ug} / \mathrm{L}$ & & $06 / 06 / 1715: 43$ & 1 \\
\hline trans-1,3-Dichloropropene & 0.50 & $U$ & 0.50 & & ug/L & & 06/06/17 15:43 & 1 \\
\hline 1,1,2-Trichloroethane & 0.50 & $U$ & 0.50 & & ug/L & & 06/06/17 15:43 & 1 \\
\hline Tetrachloroethene & 0.50 & $\mathrm{U}$ & 0.50 & & $\mathrm{ug} / \mathrm{L}$ & & $06 / 06 / 1715: 43$ & 1 \\
\hline 2-Hexanone & 5.0 & $U$ & 5.0 & & ug/L & & 06/06/17 15:43 & 1 \\
\hline Dibromochloromethane & 0.50 & $U$ & 0.50 & & ug/L & & 06/06/17 15:43 & 1 \\
\hline 1,2-Dibromoethane & 0.50 & $\mathrm{U}$ & 0.50 & & $\mathrm{ug} / \mathrm{L}$ & & $06 / 06 / 1715: 43$ & 1 \\
\hline Chlorobenzene & 0.50 & $U$ & 0.50 & & ug/L & & 06/06/17 15:43 & 1 \\
\hline Ethylbenzene & 0.50 & $U$ & 0.50 & & ug/L & & 06/06/17 15:43 & 1 \\
\hline o-Xylene & 0.50 & $U$ & 0.50 & & $\mathrm{ug} / \mathrm{L}$ & & $06 / 06 / 1715: 43$ & 1 \\
\hline m,p-Xylene & 0.50 & $U$ & 0.50 & & ug/L & & 06/06/17 15:43 & 1 \\
\hline Styrene & 0.50 & $U$ & 0.50 & & ug/L & & 06/06/17 15:43 & 1 \\
\hline Bromoform & 0.50 & $\mathrm{U}$ & 0.50 & & $\mathrm{ug} / \mathrm{L}$ & & $06 / 06 / 1715: 43$ & 1 \\
\hline Isopropylbenzene & 0.50 & $U$ & 0.50 & & ug/L & & 06/06/17 15:43 & 1 \\
\hline 1,1,2,2-Tetrachloroethane & 0.50 & $U$ & 0.50 & & ug/L & & 06/06/17 15:43 & 1 \\
\hline 1,3-Dichlorobenzene & 0.50 & $\mathrm{U}$ & 0.50 & & $\mathrm{ug} / \mathrm{L}$ & & $06 / 06 / 1715: 43$ & 1 \\
\hline 1,4-Dichlorobenzene & 0.50 & $U$ & 0.50 & & ug/L & & 06/06/17 15:43 & 1 \\
\hline 1,2-Dichlorobenzene & 0.50 & $U$ & 0.50 & & ug/L & & 06/06/17 15:43 & 1 \\
\hline
\end{tabular}

Client Sample ID: Method Blank Prep Type: Total/NA

TestAmerica Burlington 


\section{Method: SOM02.2/VOA_Tr - Trace Water (Continued)}

Lab Sample ID: MB 200-117296/7

Matrix: Water

Analysis Batch: 117296
Client Sample ID: Method Blank Prep Type: Total/NA

\begin{tabular}{|c|c|c|c|c|c|c|c|c|c|}
\hline Analyte & $\begin{array}{r}\text { MB } \\
\text { Result }\end{array}$ & $\begin{array}{l}\text { MB } \\
\text { Qualifier }\end{array}$ & $\mathbf{R L}$ & MDLE & Unit & D & Prepared & Analyzed & Dil Fac \\
\hline 1,2-Dibromo-3-chloropropane & 0.50 & $\overline{\mathrm{U}}$ & 0.50 & & $\overline{\mathrm{ug} / \mathrm{L}}$ & & & $\overline{06 / 06 / 17 ~ 15: 43}$ & \\
\hline $1,2,4$-Trichlorobenzene & 0.16 & $\mathrm{~J}$ & 0.50 & & $\mathrm{ug} / \mathrm{L}$ & & & $06 / 06 / 1715: 43$ & \\
\hline 1,2,3-Trichlorobenzene & 0.29 & $\mathrm{~J}$ & 0.50 & & $\mathrm{ug} / \mathrm{L}$ & & & 06/06/17 15:43 & \\
\hline
\end{tabular}

MB MB

Tentatively Identified Compound

Unknown Est. Result Qualifier

Total Alkanes

Unit
ug/L $/ L$

$\underline{D}$

$\frac{R T}{7.28} \frac{\text { CAS No. }}{\text { STLO0989 }} \stackrel{\text { Prepared }}{ }$

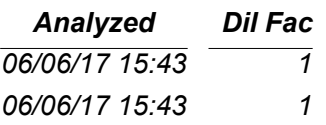

MB MB

Surrogate \%Recovery Qualifie 100 Limits

Vinyl chloride-d3

116

Chloroethane-d5

93

129

$60-125$

2-Butanone-d5

121

$40-130$

Chloroform-d

1,2-Dichloroethane-d4

Benzene-d6

1,2-Dichloropropane-d6

136 *

114

$70-125$

$70-130$

$70-125$

$93 \quad 60-140$

$113 \quad 70-130$

trans-1,3-Dichloropropene-d4

113

$55-130$

2-Hexanone-d5

127

$45-130$

1,1,2,2-Tetrachloroethane-d2

109

$65-120$

1,2-Dichlorobenzene-d4

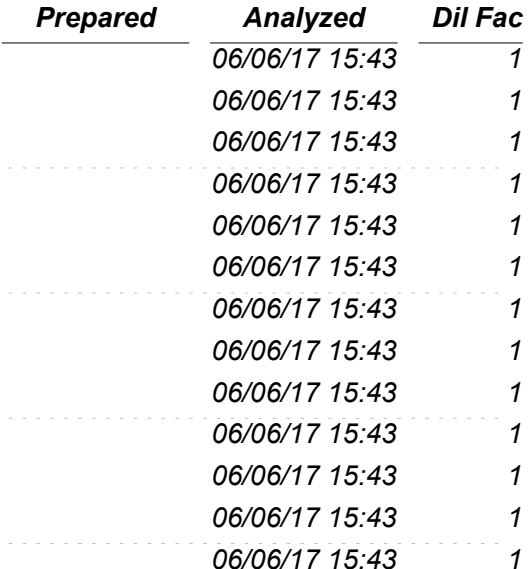

Client Sample ID: Method Blank

Prep Type: Total/NA

Matrix: Water

Analysis Batch: 117409

\begin{tabular}{|c|c|c|c|c|c|c|c|c|c|}
\hline Analyte & $\begin{array}{r}\text { MB } \\
\text { Result }\end{array}$ & $\begin{array}{l}\text { MB } \\
\text { Qualifier }\end{array}$ & RL & MDLE & Unit & D & Prepared & Analyzed & Dil Fac \\
\hline Dichlorodifluoromethane & 0.50 & $\overline{\mathrm{U}}$ & 0.50 & & $\overline{u g} / \mathrm{L}$ & & & $\overline{06 / 08 / 17 ~ 16: 19}$ & 1 \\
\hline Chloromethane & 0.50 & $\mathrm{U}$ & 0.50 & & $\mathrm{ug} / \mathrm{L}$ & & & 06/08/17 16:19 & \\
\hline Vinyl chloride & 0.50 & $\mathrm{U}$ & 0.50 & & $\mathrm{ug} / \mathrm{L}$ & & & 06/08/17 16:19 & \\
\hline Bromomethane & 0.13 & $\mathrm{~J}$ & 0.50 & & $\mathrm{ug} / \mathrm{L}$ & & & $06 / 08 / 1716: 19$ & \\
\hline Chloroethane & 0.50 & $U$ & 0.50 & & ug/L & & & 06/08/17 16:19 & \\
\hline Trichlorofluoromethane & 0.50 & $\mathrm{U}$ & 0.50 & & $\mathrm{ug} / \mathrm{L}$ & & & 06/08/17 16:19 & \\
\hline 1,1-Dichloroethene & 0.50 & $\mathrm{U}$ & 0.50 & & $\mathrm{ug} / \mathrm{L}$ & & & $06 / 08 / 1716: 19$ & \\
\hline 1,1,2-Trichloro-1,2,2-trifluoroethane & 0.50 & $U$ & 0.50 & & ug/L & & & 06/08/17 16:19 & \\
\hline Acetone & 2.5 & $\mathrm{~J}$ & 5.0 & & ug/L & & & 06/08/17 16:19 & \\
\hline Carbon disulfide & 0.13 & $\mathrm{~J}$ & 0.50 & & $\mathrm{ug} / \mathrm{L}$ & & & $06 / 08 / 1716: 19$ & \\
\hline Methyl acetate & 0.50 & $\mathrm{U}$ & 0.50 & & ug/L & & & 06/08/17 16:19 & \\
\hline Methylene Chloride & 0.50 & $U$ & 0.50 & & $\mathrm{ug} / \mathrm{L}$ & & & 06/08/17 16:19 & \\
\hline trans-1,2-Dichloroethene & 0.50 & $\mathrm{U}$ & 0.50 & & $\mathrm{ug} / \mathrm{L}$ & & & $06 / 08 / 1716: 19$ & \\
\hline Methyl tert-butyl ether & 0.50 & $U$ & 0.50 & & ug/L & & & 06/08/17 16:19 & \\
\hline 1,1-Dichloroethane & 0.50 & $U$ & 0.50 & & ug/L & & & 06/08/17 16:19 & \\
\hline cis-1,2-Dichloroethene & 0.50 & $\mathrm{U}$ & 0.50 & & $\mathrm{ug} / \mathrm{L}$ & & & $06 / 08 / 1716: 19$ & \\
\hline 2-Butanone & 5.0 & $\mathrm{U}$ & 5.0 & & $\mathrm{ug} / \mathrm{L}$ & & & 06/08/17 16:19 & \\
\hline Bromochloromethane & 0.50 & $\mathrm{U}$ & 0.50 & & $\mathrm{ug} / \mathrm{L}$ & & & 06/08/17 16:19 & \\
\hline Chloroform & 0.50 & $\mathrm{U}$ & 0.50 & & $\mathrm{ug} / \mathrm{L}$ & & & $06 / 08 / 1716: 19$ & \\
\hline
\end{tabular}




\section{Method: SOM02.2/VOA_Tr - Trace Water (Continued)}

Lab Sample ID: MB 200-117409/6

Matrix: Water

Analysis Batch: 117409

\begin{tabular}{|c|c|c|c|c|c|c|c|}
\hline Analyte & $\begin{array}{r}\text { MB } \\
\text { Result }\end{array}$ & $\begin{array}{l}\text { MB } \\
\text { Qualifier }\end{array}$ & $\mathbf{R L}$ & MDLE & Unit & D & Prepared \\
\hline 1,1,1-Trichloroethane & 0.50 & $\bar{U}$ & 0.50 & & $\overline{u g / L}$ & & \\
\hline Cyclohexane & 0.50 & $\mathrm{U}$ & 0.50 & & $\mathrm{ug} / \mathrm{L}$ & & \\
\hline Carbon tetrachloride & 0.50 & $\mathrm{U}$ & 0.50 & & $\mathrm{ug} / \mathrm{L}$ & & \\
\hline Benzene & 0.50 & $\mathrm{U}$ & 0.50 & & $\mathrm{ug} / \mathrm{L}$ & & \\
\hline 1,2-Dichloroethane & 0.50 & $U$ & 0.50 & & ug/L & & \\
\hline Trichloroethene & 0.50 & $\mathrm{U}$ & 0.50 & & $\mathrm{ug} / \mathrm{L}$ & & \\
\hline Methylcyclohexane & 0.50 & $U$ & 0.50 & & $\mathrm{ug} / \mathrm{L}$ & & \\
\hline 1,2-Dichloropropane & 0.50 & $U$ & 0.50 & & ug/L & & \\
\hline Bromodichloromethane & 0.50 & $\mathrm{U}$ & 0.50 & & $\mathrm{ug} / \mathrm{L}$ & & \\
\hline cis-1,3-Dichloropropene & 0.50 & $U$ & 0.50 & & ug/L & & \\
\hline 4-Methyl-2-pentanone & 5.0 & $U$ & 5.0 & & ug/L & & \\
\hline Toluene & 0.50 & $\mathrm{U}$ & 0.50 & & $\mathrm{ug} / \mathrm{L}$ & & \\
\hline trans-1,3-Dichloropropene & 0.50 & $U$ & 0.50 & & ug/L & & \\
\hline 1,1,2-Trichloroethane & 0.50 & $U$ & 0.50 & & ug/L & & \\
\hline Tetrachloroethene & 0.50 & $\mathrm{U}$ & 0.50 & & $\mathrm{ug} / \mathrm{L}$ & & \\
\hline 2-Hexanone & 5.0 & $U$ & 5.0 & & ug/L & & \\
\hline Dibromochloromethane & 0.50 & $U$ & 0.50 & & ug/L & & \\
\hline 1,2-Dibromoethane & 0.50 & $\mathrm{U}$ & 0.50 & & $\mathrm{ug} / \mathrm{L}$ & & \\
\hline Chlorobenzene & 0.50 & $U$ & 0.50 & & ug/L & & \\
\hline Ethylbenzene & 0.50 & $U$ & 0.50 & & ug/L & & \\
\hline o-Xylene & 0.50 & $\mathrm{U}$ & 0.50 & & $\mathrm{ug} / \mathrm{L}$ & & \\
\hline m,p-Xylene & 0.50 & $U$ & 0.50 & & ug/L & & \\
\hline Styrene & 0.50 & $\mathrm{U}$ & 0.50 & & ug/L & & \\
\hline Bromoform & 0.50 & $\mathrm{U}$ & 0.50 & & $\mathrm{ug} / \mathrm{L}$ & & \\
\hline Isopropylbenzene & 0.50 & $U$ & 0.50 & & ug/L & & \\
\hline 1,1,2,2-Tetrachloroethane & 0.50 & $U$ & 0.50 & & ug/L & & \\
\hline 1,3-Dichlorobenzene & 0.50 & $\mathrm{U}$ & 0.50 & & $\mathrm{ug} / \mathrm{L}$ & & \\
\hline 1,4-Dichlorobenzene & 0.078 & $\mathrm{~J}$ & 0.50 & & ug/L & & \\
\hline 1,2-Dichlorobenzene & 0.50 & $U$ & 0.50 & & ug/L & & \\
\hline 1,2-Dibromo-3-chloropropane & 0.50 & $\mathrm{U}$ & 0.50 & & $\mathrm{ug} / \mathrm{L}$ & & \\
\hline 1,2,4-Trichlorobenzene & 0.30 & $\mathrm{~J}$ & 0.50 & & ug/L & & \\
\hline 1,2,3-Trichlorobenzene & 0.48 & $\mathrm{~J}$ & 0.50 & & ug/L & & \\
\hline
\end{tabular}

Client Sample ID: Method Blank Prep Type: Total/NA

\begin{tabular}{|c|c|}
\hline Analyzed & Dil Fac \\
\hline$\overline{06 / 08 / 17 ~ 16: 19}$ & 1 \\
\hline 06/08/17 16:19 & 1 \\
\hline 06/08/17 16:19 & 1 \\
\hline 06/08/17 16:19 & 1 \\
\hline 06/08/17 16:19 & 1 \\
\hline 06/08/17 16:19 & 1 \\
\hline 06/08/17 16:19 & 1 \\
\hline 06/08/17 16:19 & 1 \\
\hline 06/08/17 16:19 & 1 \\
\hline 06/08/17 16:19 & 1 \\
\hline 06/08/17 16:19 & 1 \\
\hline 06/08/17 16:19 & 1 \\
\hline 06/08/17 16:19 & 1 \\
\hline 06/08/17 16:19 & 1 \\
\hline 06/08/17 16:19 & 1 \\
\hline 06/08/17 16:19 & 1 \\
\hline 06/08/17 16:19 & 1 \\
\hline 06/08/17 16:19 & 1 \\
\hline 06/08/17 16:19 & 1 \\
\hline 06/08/17 16:19 & 1 \\
\hline 06/08/17 16:19 & 1 \\
\hline 06/08/17 16:19 & 1 \\
\hline 06/08/17 16:19 & 1 \\
\hline 06/08/17 16:19 & 1 \\
\hline 06/08/17 16:19 & 1 \\
\hline 06/08/17 16:19 & 1 \\
\hline 06/08/17 16:19 & 1 \\
\hline 06/08/17 16:19 & 1 \\
\hline 06/08/17 16:19 & 1 \\
\hline 06/08/17 16:19 & 1 \\
\hline 06/08/17 16:19 & 1 \\
\hline 06/08/17 16:19 & 1 \\
\hline
\end{tabular}

Tentatively Identified Compound

Unknown

Total Alkanes

Est. Result Qualifier

$3.1 \mathrm{~J}$

MB MB

Surrogate

Vinyl chloride-d3

Chloroethane-d5

1,1-Dichloroethene-d2

2-Butanone-d5

Chloroform-d

1,2-Dichloroethane-d4

Benzene-d6

1,2-Dichloropropane-d6

Toluene-d8

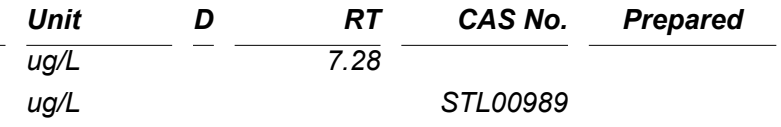

$\begin{array}{lr}\frac{\text { Analyzed }}{06 / 08 / 17 ~ 16: 19} & \text { Dil Fac } \\ & 1\end{array}$

\begin{tabular}{|c|c|}
\hline Prepared & Analyzed \\
\hline & $\overline{06 / 08 / 1716: 19}$ \\
\hline & 06/08/17 16:19 \\
\hline & $06 / 08 / 17$ 16:19 \\
\hline & $06 / 08 / 1716: 19$ \\
\hline & 06/08/17 16:19 \\
\hline & 06/08/17 16:19 \\
\hline & $06 / 08 / 1716: 19$ \\
\hline & $06 / 08 / 17$ 16:19 \\
\hline & 06/08/17 16:19 \\
\hline
\end{tabular}

\begin{tabular}{|c|c|c|}
\hline very & Qualifier & Limits \\
\hline 95 & & $40-130$ \\
\hline 100 & & $65-130$ \\
\hline 75 & & $60-125$ \\
\hline 113 & & $40-130$ \\
\hline 100 & & $70-125$ \\
\hline 107 & & $70-130$ \\
\hline 107 & & $70-125$ \\
\hline 87 & & $60-140$ \\
\hline 108 & & $70-130$ \\
\hline
\end{tabular}




\section{Method: SOM02.2/VOA_Tr - Trace Water (Continued)}

Lab Sample ID: MB 200-117409/6

Matrix: Water

Analysis Batch: 117409

\begin{tabular}{|c|c|c|c|}
\hline Surrogate & $\begin{array}{r}M B \\
\% R e c o v e r y\end{array}$ & $\begin{array}{l}\text { MB } \\
\text { Qualifier }\end{array}$ & Limits \\
\hline trans-1,3-Dichloropropene-d4 & 112 & & $55-130$ \\
\hline 2-Hexanone-d5 & 136 & * & $45-130$ \\
\hline $1,1,2,2$-Tetrachloroethane-d2 & 101 & & $65-120$ \\
\hline 1,2-Dichlorobenzene-d4 & 108 & & $80-120$ \\
\hline
\end{tabular}

Client Sample ID: Method Blank Prep Type: Total/NA

\begin{tabular}{|c|c|c|}
\hline Prepared & Analyzed & Dil Fac \\
\hline & $\overline{06 / 08 / 1716: 19}$ & $\overline{1}$ \\
\hline & 06/08/17 16:19 & 1 \\
\hline & 06/08/17 16:19 & 1 \\
\hline & $06 / 08 / 1716: 19$ & 1 \\
\hline
\end{tabular}




\section{QC Association Summary}

Client: Argonne National Laboratory

\section{GC/MS VOA}

\section{Analysis Batch: 117296}

\begin{tabular}{|c|c|c|c|c|c|}
\hline Lab Sample ID & Client Sample ID & Prep Type & Matrix & Method & Prep Batch \\
\hline $200-38792-1$ & MRMW5S-W-39794 & Total/NA & Water & $\begin{array}{l}\text { SOM02.2/VOA } \\
\mathrm{Tr}\end{array}$ & \\
\hline 200-38792-2 & MRMW10S-W-39799 & Total/NA & Water & $\begin{array}{l}\text { SOM02.2/VOA } \\
\mathrm{Tr}\end{array}$ & \\
\hline 200-38792-3 & MRMW4S-W-39793 & Total/NA & Water & $\begin{array}{l}\text { SOM02.2/VOA } \\
\mathrm{Tr}\end{array}$ & \\
\hline $200-38792-4$ & MRQCTB-W-39813 & Total/NA & Water & $\begin{array}{l}\text { SOM02.2/VOA } \\
\text { Tr }\end{array}$ & \\
\hline $200-38792-5$ & VHBLK01 & Total/NA & Water & $\begin{array}{l}\text { SOM02.2/VOA } \\
\mathrm{Tr}\end{array}$ & \\
\hline MB 200-117296/7 & Method Blank & Total/NA & Water & $\begin{array}{l}\text { SOM02.2/VOA_ } \\
\text { Tr }\end{array}$ & \\
\hline \multicolumn{6}{|c|}{ Analysis Batch: 117409} \\
\hline Lab Sample ID & Client Sample ID & Prep Type & Matrix & Method & Prep Batch \\
\hline $200-38792-4$ & MRQCTB-W-39813 & Total/NA & Water & $\begin{array}{l}\text { SOM02.2/VOA_ } \\
\mathrm{Tr}\end{array}$ & \\
\hline MB 200-117409/6 & Method Blank & Total/NA & Water & $\begin{array}{l}\text { SOM02.2/VOA } \\
\mathrm{Tr}\end{array}$ & \\
\hline
\end{tabular}




\section{Lab Chronicle}

Client Sample ID: MRMW5S-W-39794

Lab Sample ID: 200-38792-1

Date Collected: 06/01/17 15:00

Matrix: Water

Date Received: 06/03/17 09:20

\begin{tabular}{|c|c|c|c|c|c|c|c|c|}
\hline Prep Type & $\begin{array}{l}\text { Batch } \\
\text { Type }\end{array}$ & $\begin{array}{l}\text { Batch } \\
\text { Method }\end{array}$ & Run & $\begin{array}{r}\text { Dilution } \\
\text { Factor }\end{array}$ & $\begin{array}{r}\text { Batch } \\
\text { Number }\end{array}$ & $\begin{array}{c}\text { Prepared } \\
\text { or Analyzed }\end{array}$ & Analyst & Lab \\
\hline Total/NA & Analysis & SOM02.2/VOA_Tr & & $\overline{1}$ & 117296 & $\overline{06 / 06 / 17 ~ 17: 59}$ & $\overline{\mathrm{K} 1 \mathrm{P}}$ & TAL BUR \\
\hline
\end{tabular}

\section{Client Sample ID: MRMW10S-W-39799}

Lab Sample ID: 200-38792-2

Date Collected: 06/01/17 17:15

Matrix: Water

Date Received: 06/03/17 09:20

\begin{tabular}{|c|c|c|c|c|c|c|c|c|}
\hline Prep Type & $\begin{array}{l}\text { Batch } \\
\text { Type }\end{array}$ & $\begin{array}{l}\text { Batch } \\
\text { Method }\end{array}$ & Run & $\begin{array}{r}\text { Dilution } \\
\text { Factor }\end{array}$ & $\begin{array}{r}\text { Batch } \\
\text { Number }\end{array}$ & $\begin{array}{l}\text { Prepared } \\
\text { or Analyzed }\end{array}$ & Analyst & Lab \\
\hline otal/NA & Analysis & SOM02.2/VOA_Tr & & $\overline{1}$ & 117296 & $\overline{06 / 06 / 1718: 25}$ & $\overline{\mathrm{K} 1 \mathrm{P}}$ & $\overline{\text { TAL BUR }}$ \\
\hline
\end{tabular}

Client Sample ID: MRMW4S-W-39793

Date Collected: 06/02/17 10:50

Lab Sample ID: 200-38792-3

Date Received: 06/03/17 09:20

\begin{tabular}{|c|c|c|c|c|c|c|c|c|}
\hline Prep Type & $\begin{array}{l}\text { Batch } \\
\text { Type }\end{array}$ & $\begin{array}{l}\text { Batch } \\
\text { Method }\end{array}$ & Run & $\begin{array}{r}\text { Dilution } \\
\text { Factor }\end{array}$ & $\begin{array}{r}\text { Batch } \\
\text { Number }\end{array}$ & $\begin{array}{c}\text { Prepared } \\
\text { or Analyzed }\end{array}$ & Analyst & Lab \\
\hline Total/NA & Analysis & SOM02.2/VOA_Tr & & $\overline{1}$ & 117296 & $\overline{06 / 06 / 1718: 51}$ & $\overline{\mathrm{K} 1 \mathrm{P}}$ & $\overline{T A L ~ B U R}$ \\
\hline
\end{tabular}

\section{Client Sample ID: MRQCTB-W-39813}

Lab Sample ID: 200-38792-4

Date Collected: 06/02/17 10:00

Matrix: Water

Date Received: 06/03/17 09:20

\begin{tabular}{|c|c|c|c|c|c|c|c|c|}
\hline Prep Type & $\begin{array}{l}\text { Batch } \\
\text { Type }\end{array}$ & $\begin{array}{l}\text { Batch } \\
\text { Method }\end{array}$ & Run & $\begin{array}{l}\text { Dilution } \\
\text { Factor }\end{array}$ & $\begin{array}{r}\text { Batch } \\
\text { Number }\end{array}$ & $\begin{array}{c}\text { Prepared } \\
\text { or Analyzed }\end{array}$ & Analyst & Lab \\
\hline Total/NA & Analysis & SOM02.2/VOA_Tr & & $\overline{1}$ & 117296 & $\overline{06 / 06 / 17 ~ 19: 17}$ & $\overline{\mathrm{K} 1 \mathrm{P}}$ & $\overline{T A L}$ BUR \\
\hline Total/NA & Analysis & SOM02.2NOA_Tr & & 1 & 117409 & 06/08/17 22:50 & $\mathrm{P} 1 \mathrm{M}$ & TAL BUR \\
\hline
\end{tabular}

\section{Client Sample ID: VHBLK01}

Lab Sample ID: 200-38792-5

Date Collected: 06/03/17 11:08

Matrix: Water

Date Received: 06/03/17 09:20

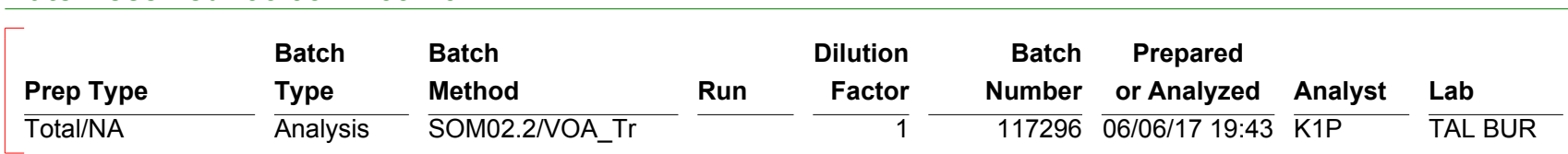

Laboratory References:

TAL BUR = TestAmerica Burlington, 30 Community Drive, Suite 11, South Burlington, VT 05403, TEL (802)660-1990 


\section{Accreditation/Certification Summary}

Client: Argonne National Laboratory

TestAmerica Job ID: 200-38792-1

Project/Site: Morrill, KS (200-38792)

SDG: $200-38792-1$

\section{Laboratory: TestAmerica Burlington}

All accreditations/certifications held by this laboratory are listed. Not all accreditations/certifications are applicable to this report.

\begin{tabular}{|c|c|c|c|c|}
\hline Authority & Program & EPA Region & Identification Number & Expiration Date \\
\hline Connecticut & State Program & $\overline{1}$ & $\overline{\mathrm{PH}}-0751$ & $09-30-17$ \\
\hline DE Haz. Subst. Cleanup Act (HSCA) & State Program & 3 & NA & $02-02-18$ \\
\hline Florida & NELAP & 4 & E87467 & $06-30-17$ * \\
\hline$L-A-B$ & DoD ELAP & & L2336 & $02-25-20$ \\
\hline Maine & State Program & 1 & VT00008 & 04-17-19 \\
\hline Minnesota & NELAP & 5 & $050-999-436$ & $12-31-17$ \\
\hline New Hampshire & NELAP & 1 & 2006 & $12-18-17$ \\
\hline New Jersey & NELAP & 2 & VT972 & $06-30-17^{*}$ \\
\hline New York & NELAP & 2 & 10391 & 04-01-18 \\
\hline Pennsylvania & NELAP & 3 & $68-00489$ & $04-30-18$ \\
\hline Rhode Island & State Program & 1 & LAO00298 & $12-30-17$ \\
\hline US Fish \& Wildlife & Federal & & LE-058448-0 & $10-31-17$ \\
\hline USDA & Federal & & P330-11-00093 & $12-05-19$ \\
\hline Vermont & State Program & 1 & VT-4000 & $12-31-17$ \\
\hline Virginia & NELAP & 3 & 460209 & $12-14-17$ \\
\hline
\end{tabular}

${ }^{*}$ Accreditation/Certification renewal pending - accreditation/certification considered valid 


\section{Method Summary}

Client: Argonne National Laboratory

TestAmerica Job ID: 200-38792-1

Project/Site: Morrill, KS (200-38792)

Method Description

SOM02.2/VOA_Tr Trace Water

Protocol References:

SOM02.2 $=$ SOM02.2

Laboratory References:

TAL BUR = TestAmerica Burlington, 30 Community Drive, Suite 11, South Burlington, VT 05403, TEL (802)660-1990
Laboratory

Protocol

TAL BUR 


\section{Sample Summary}

Client: Argonne National Laboratory

TestAmerica Job ID: 200-38792-1 Project/Site: Morrill, KS (200-38792)

Lab Sample ID 200-38792-1 200-38792-2

200-38792-3

200-38792-4

200-38792-5
Client Sample ID MRMW5S-W-39794 MRMW10S-W-39799 MRMW4S-W-39793

MRQCTB-W-39813

VHBLK01

\begin{tabular}{l} 
Matrix \\
\hline Water \\
Water \\
Water \\
Water \\
Water
\end{tabular}

Collected

Received 06/01/17 15:00 06/03/17 09:20 06/01/17 17:15 06/03/17 09:20 06/02/17 10:50 06/03/17 09:20 06/02/17 10:00 06/03/17 09:20 06/03/17 11:08 06/03/17 09:20 
Lab Name: TestAmerica Burlington SDG No.: 200-38792-1

Instrument ID: CHD.i

Lab Sample ID: CCVC 200-117409/22

Date Analyzed: 06/08/17 23:16

\begin{tabular}{|l|c|c|c|c|}
\hline \multicolumn{1}{|c|}{ COMPOUND NAME } & RETENTION & \multicolumn{2}{|c|}{ MANUAL INTEGRATION } \\
\cline { 3 - 5 } & TIME & REASON & ANALYST & DATE \\
\hline Bromomethane & 2.03 & Incomplete Integration & $\mathrm{kirchnerb}$ & $06 / 18 / 17$ 19:55 \\
\hline
\end{tabular}

Job No.: 200-38792-1

Analysis Batch Number: 117409

Client Sample ID:

Lab File ID: $2540522 . \mathrm{D}$
GC Column: DB-624

ID $: 0.2(\mathrm{~mm})$ 
SDG No.: 200-38792-1

\begin{tabular}{|c|c|c|c|c|c|c|c|c|}
\hline \multirow[b]{2}{*}{ Reagent ID } & \multirow[b]{2}{*}{$\begin{array}{l}\text { Exp } \\
\text { Date }\end{array}$} & \multirow[b]{2}{*}{$\begin{array}{l}\text { Prep } \\
\text { Date }\end{array}$} & \multirow[b]{2}{*}{$\begin{array}{l}\text { Dilutant } \\
\text { Used }\end{array}$} & \multirow{2}{*}{$\begin{array}{l}\text { Reagent } \\
\text { Final } \\
\text { Volume }\end{array}$} & \multicolumn{2}{|c|}{ Parent Reagent } & \multirow[b]{2}{*}{ Analyte } & \multirow[b]{2}{*}{ Concentration } \\
\hline & & & & & Reagent ID & $\begin{array}{l}\text { Volume } \\
\text { Added }\end{array}$ & & \\
\hline \multirow[t]{47}{*}{ VMSOMTRCALw_00125 } & \multirow[t]{47}{*}{$06 / 22 / 17$} & \multirow[t]{47}{*}{$05 / 22 / 17$} & \multirow[t]{47}{*}{ METHANOL, Lot 157266} & \multirow[t]{47}{*}{$2000 \mathrm{uL}$} & \multirow[t]{6}{*}{ VM8260CALbs_00220 } & \multirow[t]{6}{*}{$21 \mathrm{uL}$} & Bromomethane & $21 \mathrm{ug} / \mathrm{mL}$ \\
\hline & & & & & & & Chloroethane & $21 \mathrm{ug} / \mathrm{mL}$ \\
\hline & & & & & & & Chloromethane & $21 \mathrm{ug} / \mathrm{mL}$ \\
\hline & & & & & & & Dichlorodifluoromethane & $21 \mathrm{ug} / \mathrm{mL}$ \\
\hline & & & & & & & Trichlorofluoromethane & $21 \mathrm{ug} / \mathrm{mL}$ \\
\hline & & & & & & & Vinyl chloride & $21 \mathrm{ug} / \mathrm{mL}$ \\
\hline & & & & & \multirow{41}{*}{ VMSOMCALas_00036 } & \multirow[t]{41}{*}{$21 \mathrm{uL}$} & 1,1,1-Trichloroethane & $21 \mathrm{ug} / \mathrm{mL}$ \\
\hline & & & & & & & $1,1,2,2$-Tetrachloroethane & $21 \mathrm{ug} / \mathrm{mL}$ \\
\hline & & & & & & & $\begin{array}{l}1,1,2 \text {-Trichloro-1,2,2-trifluor } \\
\text { oethane }\end{array}$ & $21 \mathrm{ug} / \mathrm{mL}$ \\
\hline & & & & & & & 1,1,2-Trichloroethane & $21 \mathrm{ug} / \mathrm{mL}$ \\
\hline & & & & & & & 1,1-Dichloroethane & $21 \mathrm{ug} / \mathrm{mL}$ \\
\hline & & & & & & & 1,1-Dichloroethene & $21 \mathrm{ug} / \mathrm{mL}$ \\
\hline & & & & & & & 1,2,3-Trichlorobenzene & $21 \mathrm{ug} / \mathrm{mL}$ \\
\hline & & & & & & & 1,2,4-Trichlorobenzene & $21 \mathrm{ug} / \mathrm{mL}$ \\
\hline & & & & & & & 1,2-Dibromo-3-chloropropane & $21 \mathrm{ug} / \mathrm{mL}$ \\
\hline & & & & & & & 1,2-Dibromoethane & $21 \mathrm{ug} / \mathrm{mL}$ \\
\hline & & & & & & & 1,2-Dichlorobenzene & $21 \mathrm{ug} / \mathrm{mL}$ \\
\hline & & & & & & & 1,2-Dichloroethane & $21 \mathrm{ug} / \mathrm{mL}$ \\
\hline & & & & & & & 1,2-Dichloropropane & $21 \mathrm{ug} / \mathrm{mL}$ \\
\hline & & & & & & & 1,3-Dichlorobenzene & $21 \mathrm{ug} / \mathrm{mL}$ \\
\hline & & & & & & & 1,4-Dichlorobenzene & $21 \mathrm{ug} / \mathrm{mL}$ \\
\hline & & & & & & & Benzene & $21 \mathrm{ug} / \mathrm{mL}$ \\
\hline & & & & & & & Bromochloromethane & $21 \mathrm{ug} / \mathrm{mL}$ \\
\hline & & & & & & & Bromodichloromethane & $21 \mathrm{ug} / \mathrm{mL}$ \\
\hline & & & & & & & Bromoform & $21 \mathrm{ug} / \mathrm{mL}$ \\
\hline & & & & & & & Carbon disulfide & $21 \mathrm{ug} / \mathrm{mL}$ \\
\hline & & & & & & & Carbon tetrachloride & $21 \mathrm{ug} / \mathrm{mL}$ \\
\hline & & & & & & & Chlorobenzene & $21 \mathrm{ug} / \mathrm{mL}$ \\
\hline & & & & & & & Chloroform & $21 \mathrm{ug} / \mathrm{mL}$ \\
\hline & & & & & & & cis-1,2-Dichloroethene & $21 \mathrm{ug} / \mathrm{mL}$ \\
\hline & & & & & & & cis-1,3-Dichloropropene & $21 \mathrm{ug} / \mathrm{mL}$ \\
\hline & & & & & & & Cyclohexane & $21 \mathrm{ug} / \mathrm{mL}$ \\
\hline & & & & & & & Dibromochloromethane & $21 \mathrm{ug} / \mathrm{mL}$ \\
\hline & & & & & & & Ethylbenzene & $21 \mathrm{ug} / \mathrm{mL}$ \\
\hline & & & & & & & Isopropylbenzene & $21 \mathrm{ug} / \mathrm{mL}$ \\
\hline & & & & & & & $\mathrm{m}, \mathrm{p}$-Xylene & $21 \mathrm{ug} / \mathrm{mL}$ \\
\hline & & & & & & & Methyl acetate & $21 \mathrm{ug} / \mathrm{mL}$ \\
\hline & & & & & & & Methyl tert-butyl ether & $21 \mathrm{ug} / \mathrm{mL}$ \\
\hline & & & & & & & Methylcyclohexane & $21 \mathrm{ug} / \mathrm{mL}$ \\
\hline & & & & & & & Methylene Chloride & $21 \mathrm{ug} / \mathrm{mL}$ \\
\hline & & & & & & & o-Xylene & $21 \mathrm{ug} / \mathrm{mL}$ \\
\hline & & & & & & & Styrene & $21 \mathrm{ug} / \mathrm{mL}$ \\
\hline & & & & & & & Tetrachloroethene & $21 \mathrm{ug} / \mathrm{mL}$ \\
\hline & & & & & & & Toluene & $21 \mathrm{ug} / \mathrm{mL}$ \\
\hline & & & & & & & trans-1,2-Dichloroethene & $21 \mathrm{ug} / \mathrm{mL}$ \\
\hline & & & & & & & trans-1,3-Dichloropropene & $21 \mathrm{ug} / \mathrm{mL}$ \\
\hline & & & & & & & Trichloroethene & $21 \mathrm{ug} / \mathrm{mL}$ \\
\hline
\end{tabular}


SDG No.: 200-38792-1

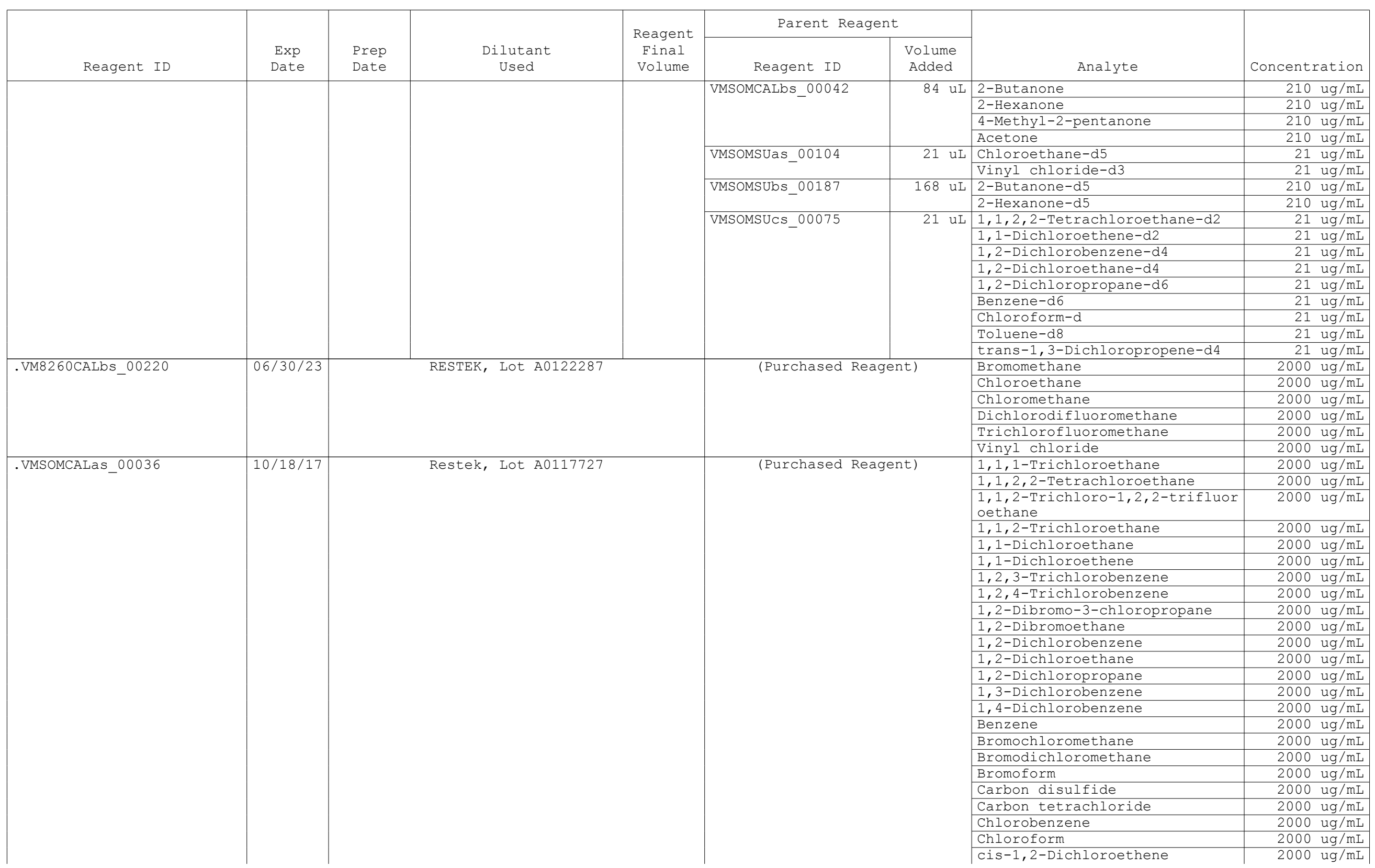


SDG No.: 200-38792-1

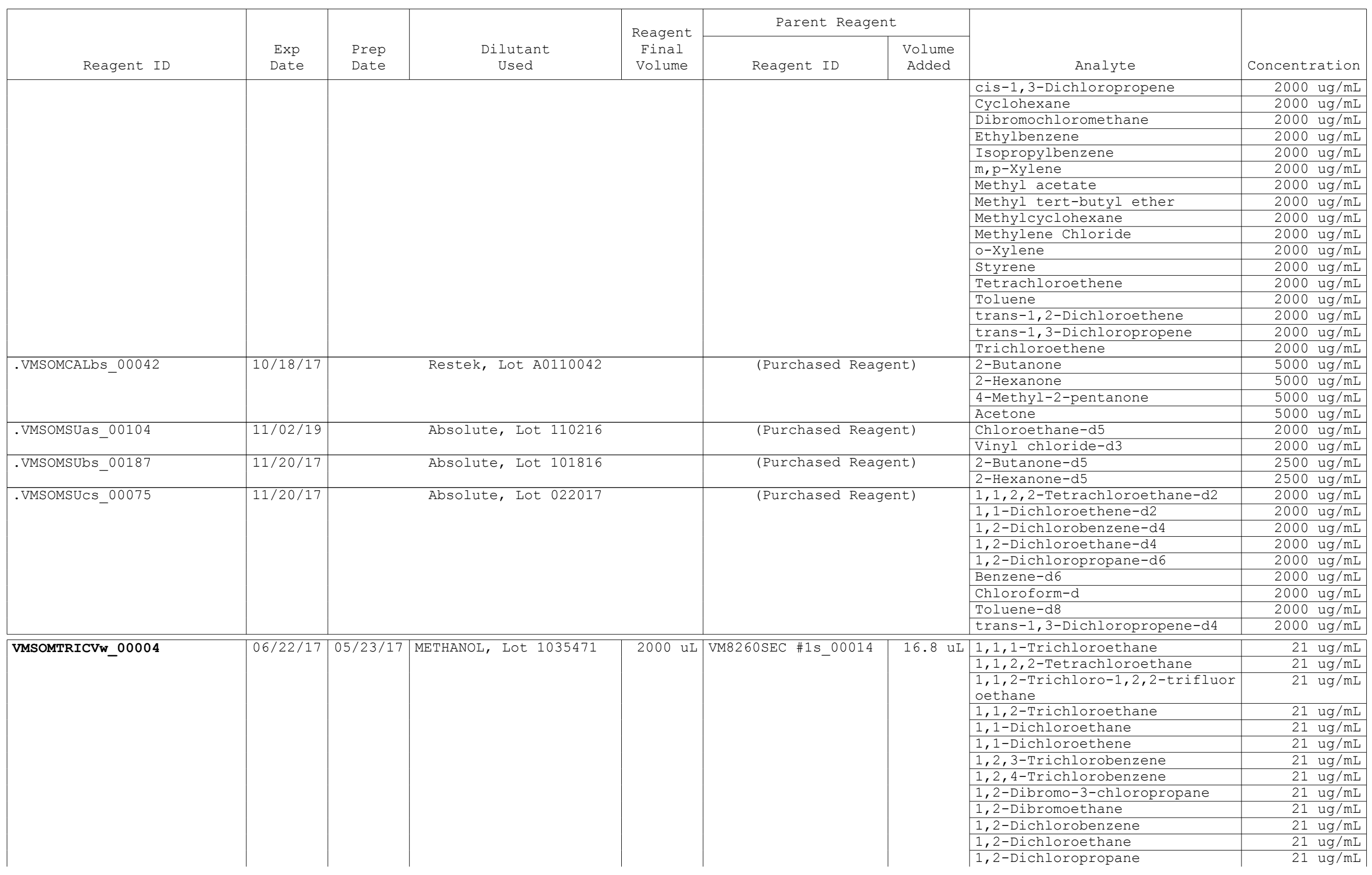


SDG No.: 200-38792-1

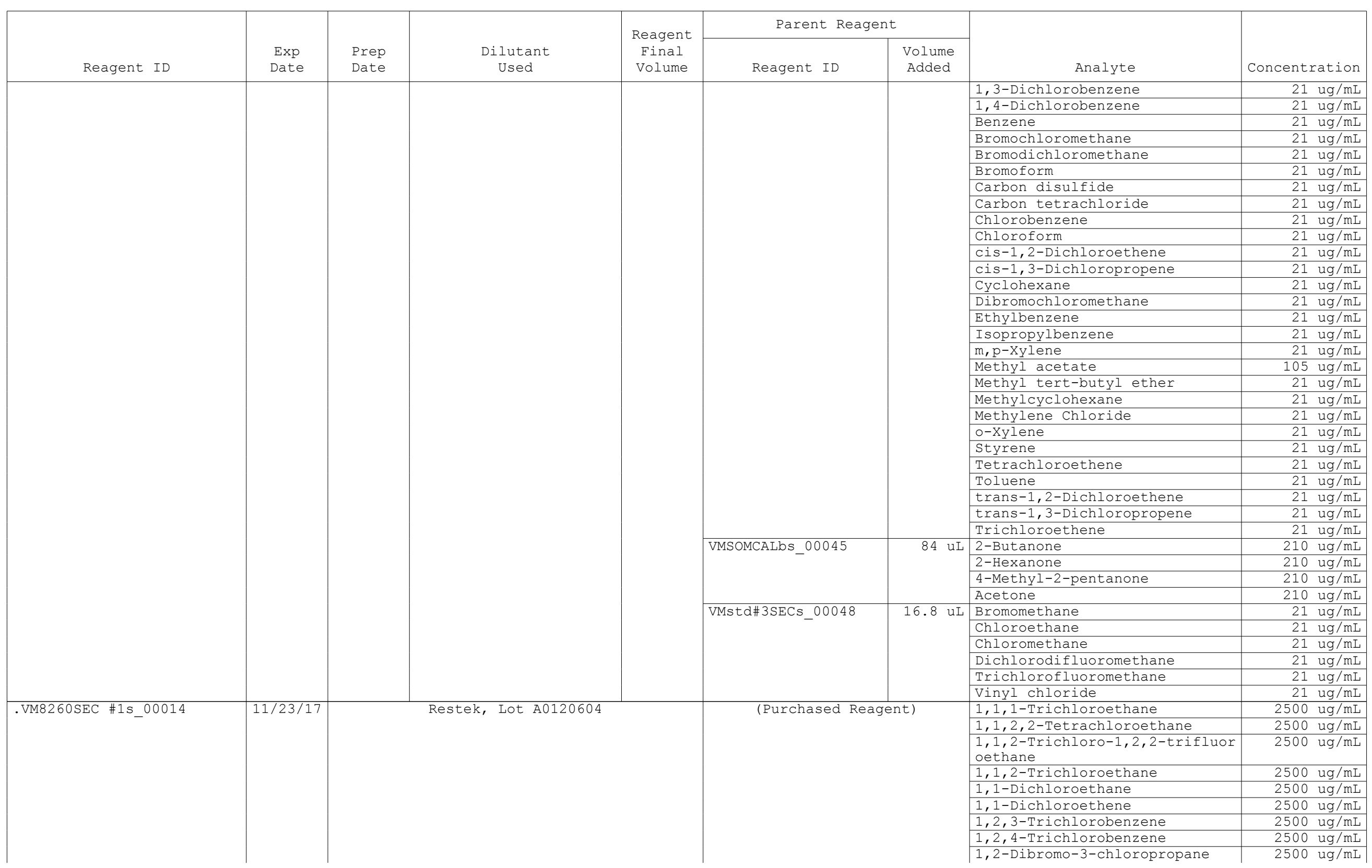


SDG No.: 200-38792-1

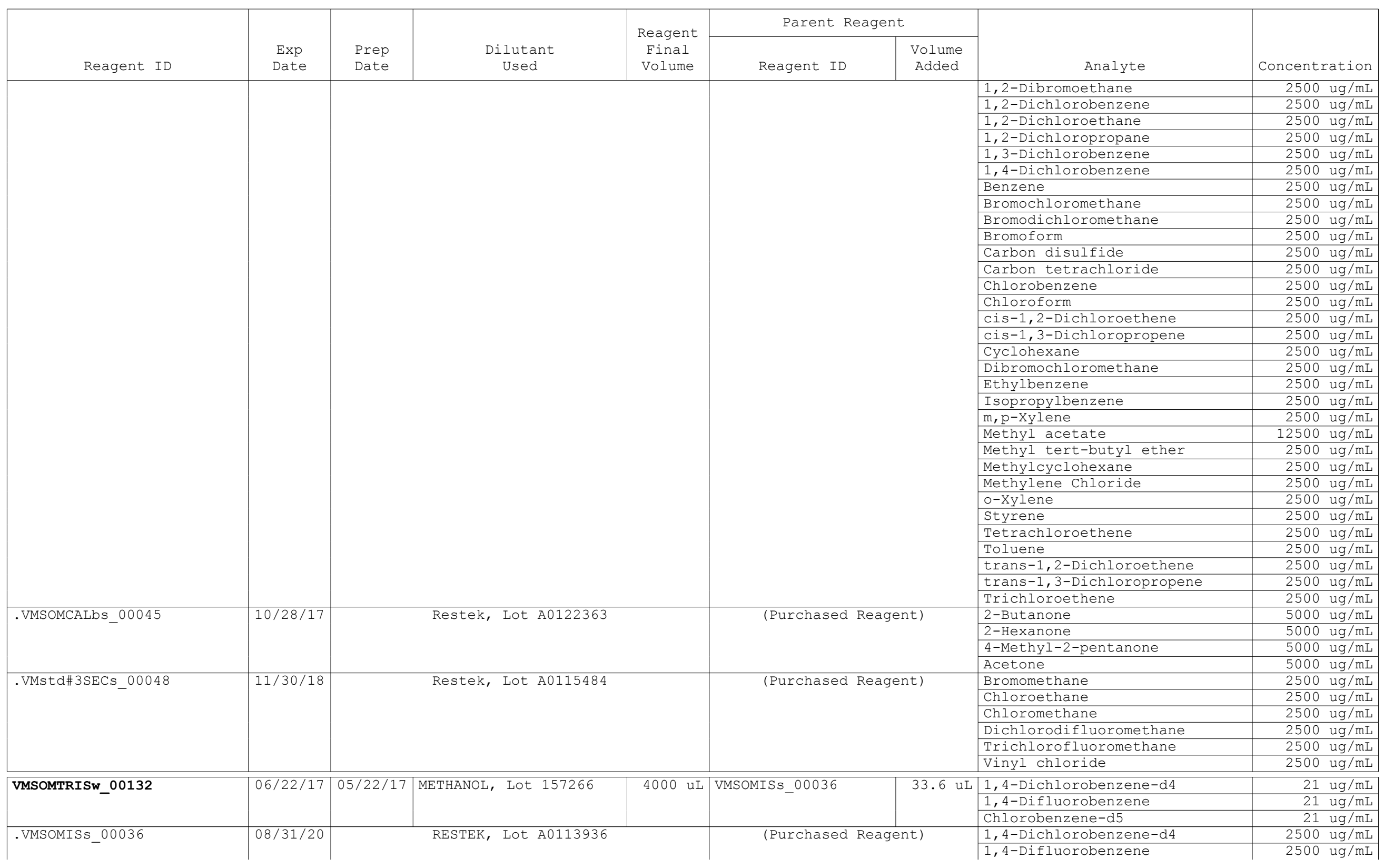


SDG No.: 200-38792-1

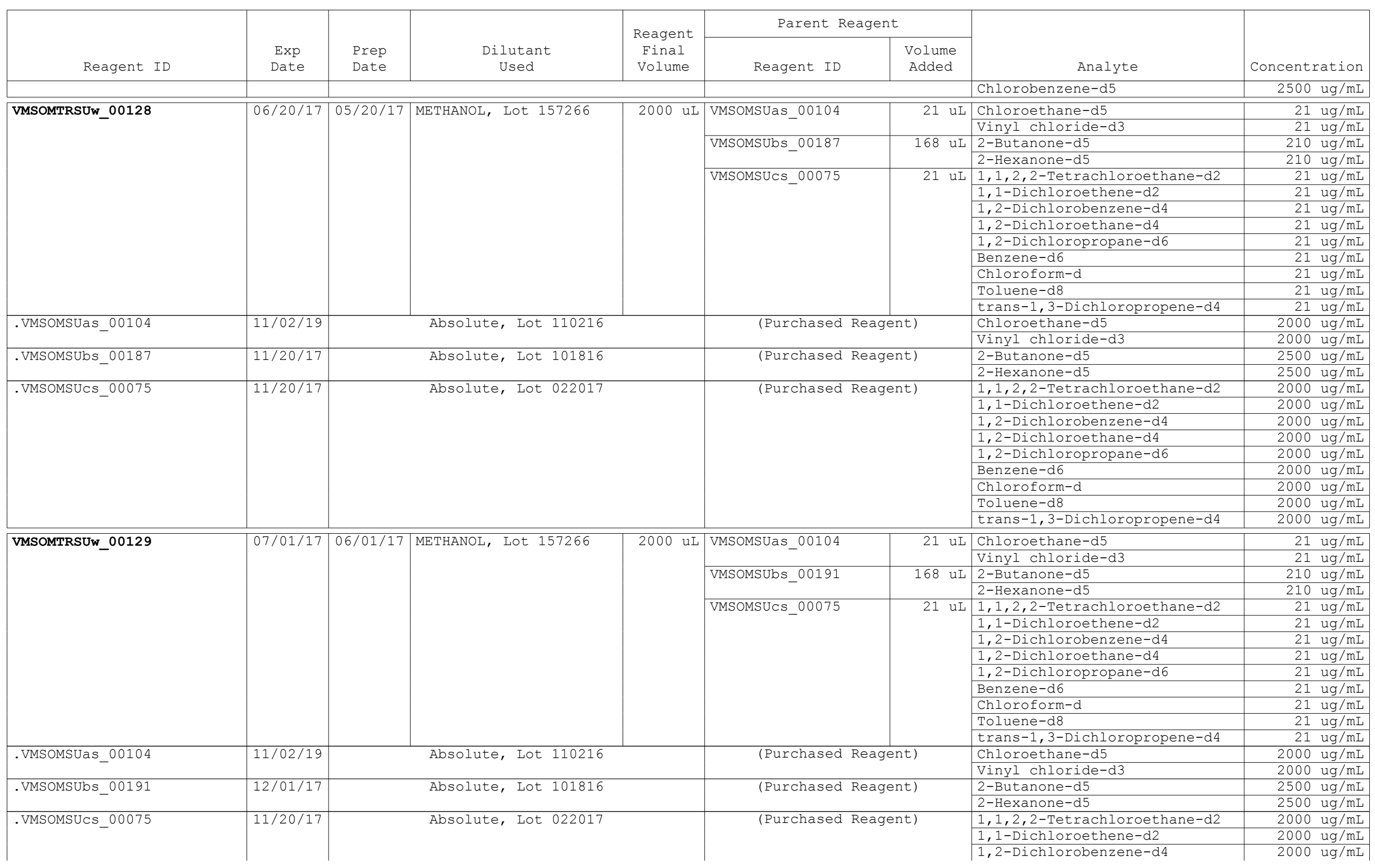


SDG No.: 200-38792-1

\begin{tabular}{|c|c|c|c|c|c|c|c|c|}
\hline \multirow[b]{2}{*}{ Reagent ID } & \multirow[b]{2}{*}{$\begin{array}{l}\text { Exp } \\
\text { Date }\end{array}$} & \multirow[b]{2}{*}{$\begin{array}{l}\text { Prep } \\
\text { Date }\end{array}$} & \multirow[b]{2}{*}{$\begin{array}{l}\text { Dilutant } \\
\text { Used }\end{array}$} & \multirow{2}{*}{$\begin{array}{c}\text { Reagent } \\
\text { Final } \\
\text { Volume }\end{array}$} & \multicolumn{2}{|c|}{ Parent Reagent } & \multirow[b]{2}{*}{ Analyte } & \multirow[b]{2}{*}{ Concentration } \\
\hline & & & & & Reagent ID & $\begin{array}{l}\text { Volume } \\
\text { Added }\end{array}$ & & \\
\hline & & & & & & & 1,2-Dichloroethane-d4 & $2000 \mathrm{ug} / \mathrm{mL}$ \\
\hline & & & & & & & 1,2-Dichloropropane-d6 & $2000 \mathrm{ug} / \mathrm{mL}$ \\
\hline & & & & & & & Benzene-d6 & $2000 \mathrm{ug} / \mathrm{mL}$ \\
\hline & & & & & & & Chloroform-d & $2000 \mathrm{ug} / \mathrm{mL}$ \\
\hline & & & & & & & Toluene-d8 & $2000 \mathrm{ug} / \mathrm{mL}$ \\
\hline & & & & & & & trans-1,3-Dichloropropene-d4 & $2000 \mathrm{ug} / \mathrm{mL}$ \\
\hline
\end{tabular}




\section{Method SOM Vol Tr}

\section{Trace Volatile Organic Compounds (GC/MS) by CLP SOM}


FORM II

GC/MS VOA SURROGATE RECOVERY

Lab Name: TestAmerica Burlington

Job No.: 200-38792-1

SDG No.: 200-38792-1

Matrix: Water

Level: Trace

GC Column (1): DB-624

ID: $0.2(\mathrm{~mm})$

\begin{tabular}{|c|c|c|c|c|c|c|c|c|c|}
\hline Client Sample ID & Lab Sample ID & $\mathrm{VCL} \quad \#$ & CLA \# & $\mathrm{DCE} \quad \#$ & BUT & CLF & $\mathrm{DCA}$ & BEN & $\mathrm{DPA}$ \\
\hline MRMW5S-W-39794 & $200-38792-1$ & 106 & 124 & 100 & 150 & 121 & 137 & 118 & 95 \\
\hline MRMW10S-W-39799 & $200-38792-2$ & 103 & 122 & 96 & 149 & 124 & 139 & 117 & 96 \\
\hline MRMW 4S-W-39793 & $200-38792-3$ & 103 & 121 & 94 & 152 & 123 & 139 & 117 & 95 \\
\hline MRQCTB-W-39813 & $200-38792-4$ & 108 & 125 & 96 & 155 & 127 & 142 & 120 & 97 \\
\hline MRQCTB-W-39813 & $200-38792-4$ & 78 & 109 & 89 & 124 & 117 & 137 & 112 & 89 \\
\hline \multirow[t]{3}{*}{ VHBLK01 } & $200-38792-5$ & 102 & 118 & 93 & 123 & 122 & 137 & 119 & 96 \\
\hline & MB 200-117296/7 & 100 & 116 & 93 & 129 & 121 & 136 & 114 & 93 \\
\hline & MB 200-117409/6 & 95 & 100 & 75 & 113 & 100 & 107 & 107 & 87 \\
\hline
\end{tabular}

VCL $=$ Vinyl chloride-d3

$\mathrm{CLA}=$ Chloroethane-d5

$\mathrm{DCE}=1,1-\mathrm{Dichloroethene- \textrm {d } 2}$

BUT $=2$-Butanone-d5

$\mathrm{CLF}=$ Chloroform-d

$\mathrm{DCA}=1,2-\mathrm{Dichloroethane- \textrm {d } 4}$

$\mathrm{BEN}=$ Benzene-d6

$\mathrm{DPA}=1,2$-Dichloropropane-d 6
QC LIMITS

$40-130$

$65-130$

$60-125$

$40-130$

$70-125$

$70-130$

$70-125$

$60-140$

\# Column to be used to flag recovery values

FORM II SOM02.2/VOA_Tr 
FORM II

GC/MS VOA SURROGATE RECOVERY

Lab Name: TestAmerica Burlington

Job No.: 200-38792-1

SDG No.: 200-38792-1

Matrix: Water

Level: Trace

GC Column (1): DB-624

ID: $0.2(\mathrm{~mm})$

\begin{tabular}{|c|c|c|c|c|c|c|}
\hline Client Sample ID & Lab Sample ID & TOL \# & TDP \# & HEX & $\mathrm{TCA} \quad \#$ & DCZ \\
\hline MRMW5S-W-39794 & $200-38792-1$ & 117 & 110 & 145 & 110 & 116 \\
\hline MRMW10S-W-39799 & $200-38792-2$ & 117 & 112 & 148 & 110 & 119 \\
\hline MRMW 4S-W-39793 & $200-38792-3$ & 116 & 111 & 150 & 113 & 120 \\
\hline MRQCTB-W-39813 & $200-38792-4$ & 119 & 115 & 158 & 115 & 119 \\
\hline MRQCTB-W-39813 & $200-38792-4$ & 112 & 110 & 132 & 109 & 117 \\
\hline \multirow[t]{3}{*}{ VHBLK01 } & $200-38792-5$ & 116 & 108 & 125 & 110 & 121 \\
\hline & MB 200-117296/7 & 113 & 113 & 127 & 109 & 113 \\
\hline & MB 200-117409/6 & 108 & 112 & 136 & 101 & 108 \\
\hline
\end{tabular}

TOL $=$ Toluene-d8

$\mathrm{TDP}=$ trans-1,3-Dichloropropene-d4

$\mathrm{HEX}=2-$ Hexanone-d5

$\mathrm{TCA}=1,1,2,2-$ Tetrachloroethane-d2

$\mathrm{DCZ}=1,2-\mathrm{Dichlorobenzene- \textrm {d } 4}$
QC LIMITS

$70-130$

$55-130$

$45-130$

$65-120$

$80-120$

\# Column to be used to flag recovery values

FORM II SOM02.2/VOA_Tr 
FORM IV

GC/MS VOA METHOD BLANK SUMMARY

Lab Name: TestAmerica Burlington

Job No. : 200-38792-1

SDG No.: 200-38792-1

Lab File ID: 25366_07.D

Matrix: Water

Instrument ID: CHD.i

GC Column: DB-624
Lab Sample ID: MB 200-117296/7

Heated Purge: (Y/N) N

Date Analyzed: 06/06/2017 15:43

THIS METHOD BLANK APPLIES TO THE FOLLOWING SAMPLES:

\begin{tabular}{|c|c|c|c|}
\hline CLIENT SAMPLE ID & LAB SAMPLE ID & $\begin{array}{c}\text { LAB } \\
\text { FILE ID }\end{array}$ & DATE ANALYZED \\
\hline MRMW5S-W-39794 & $200-38792-1$ & $2536608 . \mathrm{D}$ & $06 / 06 / 2017$ \\
\hline MRMW10S-W-39799 & $200-38792-2$ & $2536609 . \mathrm{D}$ & $06 / 06 / 2017$ \\
\hline MRMW 4S-W-39793 & $200-38792-3$ & $2536610 . \mathrm{D}$ & $06 / 06 / 2017$ \\
\hline MRQCTB-W-39813 & $200-38792-4$ & $2536611 . \mathrm{D}$ & $06 / 06 / 2017$ \\
\hline VHBLK01 & $200-38792-5$ & $2536612 . D$ & $06 / 06 / 2017$ \\
\hline
\end{tabular}


FORM IV

GC/MS VOA METHOD BLANK SUMMARY

Lab Name: TestAmerica Burlington

Job No.: 200-38792-1

SDG No.: 200-38792-1

Lab File ID: 25405_06.D

Matrix: Water

Instrument ID: CHD.i

GC Column: DB-624
Lab Sample ID: MB 200-117409/6

Heated Purge: (Y/N) N

Date Analyzed: 06/08/2017 16:19

THIS METHOD BLANK APPLIES TO THE FOLLOWING SAMPLES:

\begin{tabular}{|c|c|c|c}
\hline & & LAB & FILE ID \\
CLIENT SAMPLE ID & LAB SAMPLE ID & 25405 DATE ANALYZED \\
\hline MRQCTB-W-39813 & $200-38792-4$ & $06 / 08 / 2017$ & $22: 50$ \\
\hline
\end{tabular}


FORM V

GC/MS VOA INSTRUMENT PERFORMANCE CHECK

BROMOFLUOROBENZENE (BFB)

Lab Name: TestAmerica Burlington

Job No : 200-38792-1

SDG No.: 200-38792-1

Lab File ID: 25203_04.D

Instrument ID: CHD.i

BFB Injection Date: 05/23/2017

BFB Injection Time: 16:12

Analysis Batch No.: 116931

\begin{tabular}{|c|c|c|c|c|}
\hline $\mathrm{M} / \mathrm{E}$ & ION ABUNDANCE CRITERIA & \multicolumn{3}{|c|}{$\begin{array}{l}\text { \% RELATIVE } \\
\text { ABUNDANCE }\end{array}$} \\
\hline 50 & $15.0-40.0 \%$ of mass 95 & 17.4 & & \\
\hline 75 & $30.0-80.0 \%$ of mass 95 & 46.0 & & \\
\hline 95 & Base peak, $100 \%$ relative abundance & 100.0 & & \\
\hline 96 & $5.0-9.0 \%$ of mass 95 & 6.9 & & \\
\hline 173 & Less than 2.0\% of mass 174 & 0.6 & $(0.6)$ & 1 \\
\hline 174 & $50.0-120 \%$ of mass 95 & 99.7 & & \\
\hline 175 & $5.0-9.0 \%$ of mass 174 & 7.0 & $(7.0)$ & 1 \\
\hline 176 & $95.0-101 \%$ of mass 174 & 96.0 & $(96.3)$ & 1 \\
\hline 177 & $5.0-9.0 \%$ of mass 176 & 6.3 & $(6.6)$ & 2 \\
\hline
\end{tabular}

THIS CHECK APPLIES TO THE FOLLOWING SAMPLES, MS, MSD, BLANKS AND STANDARDS:

\begin{tabular}{|l|l|l|c|}
\hline CLIENT SAMPLE ID & LAB SAMPLE ID & LAB & DATE \\
FILE ID & TIME \\
ANALYZD ANALYED
\end{tabular}


FORM V

GC/MS VOA INSTRUMENT PERFORMANCE CHECK

BROMOFLUOROBENZENE (BFB)

Lab Name: TestAmerica Burlington

Job No : 200-38792-1

SDG No.: 200-38792-1

Lab File ID: 25366_03.D

Instrument ID: CHD.i

BFB Injection Date: 06/06/2017

BFB Injection Time: 13:46

Analysis Batch No.: 117296

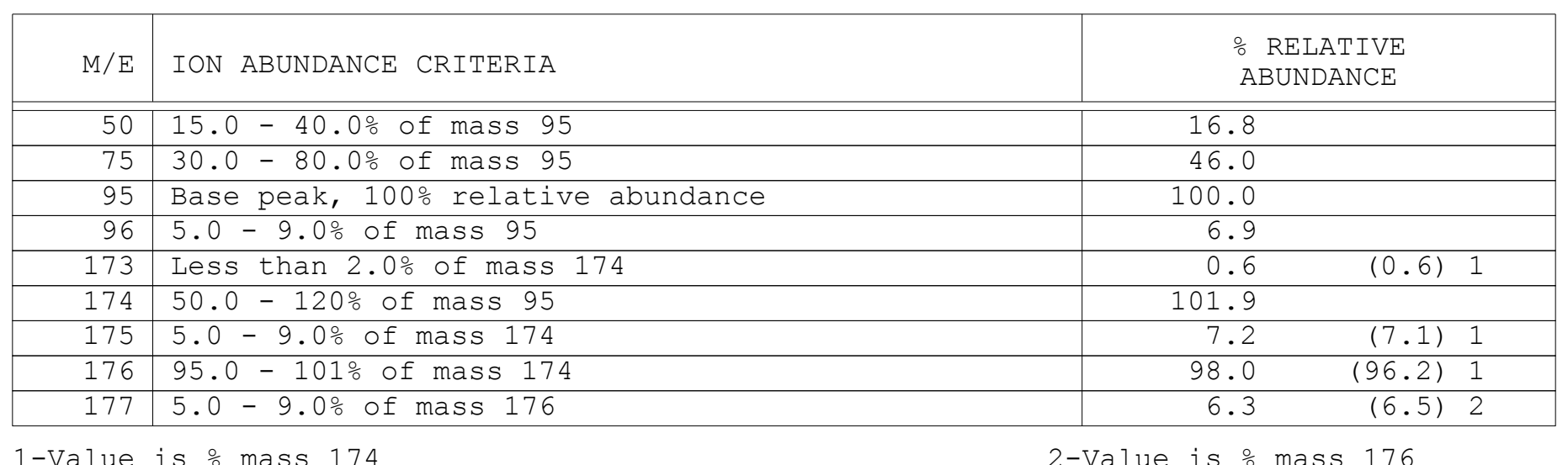

THIS CHECK APPLIES TO THE FOLLOWING SAMPLES, MS, MSD, BLANKS AND STANDARDS:

\begin{tabular}{|c|c|c|c|c|}
\hline CLIENT SAMPLE ID & LAB SAMPLE ID & $\begin{array}{l}\text { LAB } \\
\text { FILE ID }\end{array}$ & $\begin{array}{c}\text { DATE } \\
\text { ANALYZED }\end{array}$ & $\begin{array}{c}\text { TIME } \\
\text { ANALYZED }\end{array}$ \\
\hline & CCVIS 200-117296/6 & $2536606 . \mathrm{D}$ & $06 / 06 / 2017$ & $15: 17$ \\
\hline & MB 200-117296/7 & $2536607 . \mathrm{D}$ & $06 / 06 / 2017$ & $15: 43$ \\
\hline MRMW5S-W-39794 & $200-38792-1$ & $2536608 . \mathrm{D}$ & $06 / 06 / 2017$ & $17: 59$ \\
\hline MRMW10S-W-39799 & $200-38792-2$ & $2536609 . D$ & $06 / 06 / 2017$ & $18: 25$ \\
\hline MRMW 4S-W-39793 & $200-38792-3$ & $2536610 . D$ & $06 / 06 / 2017$ & $18: 51$ \\
\hline MRQCTB-W-39813 & $200-38792-4$ & $2536611 . \mathrm{D}$ & $06 / 06 / 2017$ & $19: 17$ \\
\hline \multirow[t]{3}{*}{ VHBLK01 } & $200-38792-5$ & $25366 \_12 . D$ & $06 / 06 / 2017$ & $19: 43$ \\
\hline & CCVC 200-117296/22 & $2536622 . D$ & $06 / 07 / 2017$ & $00: 03$ \\
\hline & CCVC 200-117296/23 & $2536623 . D$ & $06 / 07 / 2017$ & $00: 28$ \\
\hline
\end{tabular}


FORM V

GC/MS VOA INSTRUMENT PERFORMANCE CHECK

BROMOFLUOROBENZENE (BFB)

Lab Name: TestAmerica Burlington

Job No : 200-38792-1

SDG No.: 200-38792-1

Lab File ID: 25405_01.D

Instrument ID: CHD.i

BFB Injection Date: 06/08/2017

BFB Injection Time: 14:03

Analysis Batch No.: 117409

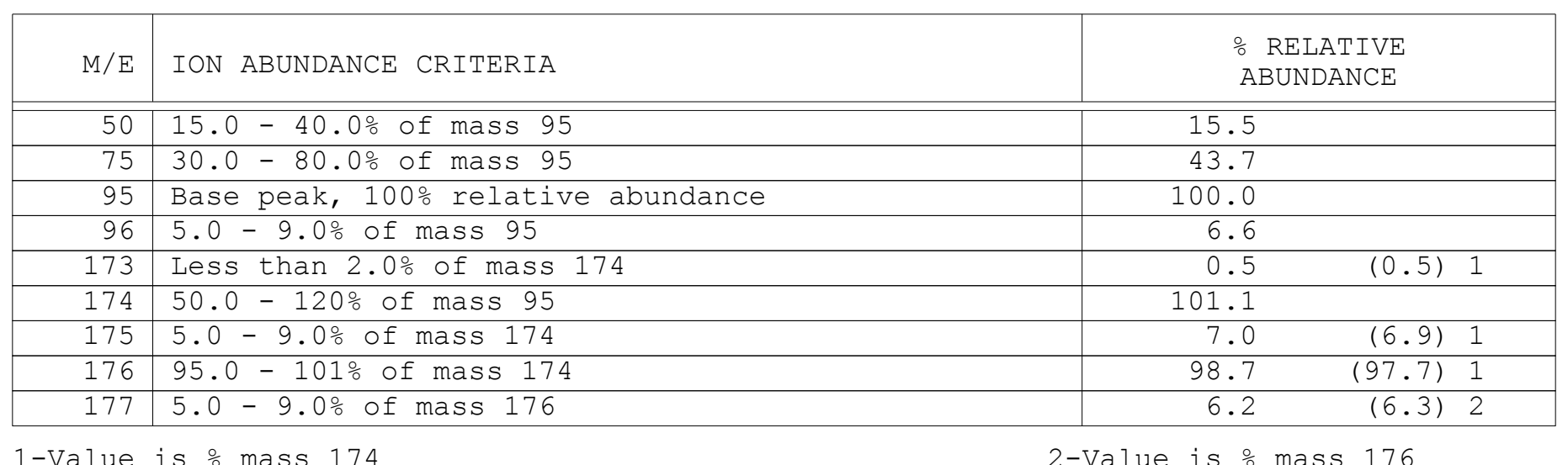

THIS CHECK APPLIES TO THE FOLLOWING SAMPLES, MS, MSD, BLANKS AND STANDARDS:

\begin{tabular}{|c|c|c|c|c|}
\hline CLIENT SAMPLE ID & LAB SAMPLE ID & $\begin{array}{l}\text { LAB } \\
\text { FILE ID }\end{array}$ & $\begin{array}{c}\text { DATE } \\
\text { ANALYZED }\end{array}$ & $\begin{array}{c}\text { TIME } \\
\text { ANALYZED }\end{array}$ \\
\hline & CCVIS 200-117409/5 & $2540505 . \mathrm{D}$ & $06 / 08 / 2017$ & $15: 53$ \\
\hline & MB 200-117409/6 & $2540506 . \mathrm{D}$ & $06 / 08 / 2017$ & $16: 19$ \\
\hline MRQCTB-W-39813 & $200-38792-4$ & $2540521 . \mathrm{D}$ & $06 / 08 / 2017$ & $22: 50$ \\
\hline & CCVC 200-117409/22 & $2540522 . D$ & $06 / 08 / 2017$ & $23: 16$ \\
\hline & CCVC 200-117409/23 & $2540523 . D$ & $06 / 08 / 2017$ & $23: 42$ \\
\hline
\end{tabular}


FORM VIII

GC/MS VOA INTERNAL STANDARD AREA AND RETENTION TIME SUMMARY

Lab Name: TestAmerica Burlington

Job No. : 200-38792-1

SDG No.: 200-38792-1

Sample No.: ICIS 200-116931/28

Instrument ID: CHD.i

Lab File ID (Standard): 25203_09.D

Date Analyzed: 05/23/2017 18:24

Calibration ID: 37252

GC Column: DB-624

ID $: 0.2(\mathrm{~mm})$

Heated Purge: (Y/N) N

\begin{tabular}{|c|c|c|c|c|c|c|c|}
\hline & & \multicolumn{2}{|c|}{ DFBZ } & \multicolumn{2}{|c|}{ CBNZd5 } & \multicolumn{2}{|c|}{$\mathrm{DCBd} 4$} \\
\hline & & AREA \# & RT \# & AREA \# & RT \# & AREA \# & RT \# \\
\hline INITIAL CALIBRATI & POINT & 2166328 & 5.95 & 1703044 & 9.31 & 818048 & 12.12 \\
\hline UPPER LIMIT & & 4332656 & 6.12 & 3406088 & 9.47 & 1636096 & 12.29 \\
\hline LOWER LIMIT & & 1083164 & 5.79 & 851522 & 9.14 & 409024 & 11.96 \\
\hline LAB SAMPLE ID & CLIENT SAMPLE ID & & & & & & \\
\hline ICV 200-116931/31 & & 2239915 & 5.95 & 1651922 & 9.31 & 943101 & 12.12 \\
\hline
\end{tabular}

DFBZ = 1,4-Difluorobenzene

CBNZd5 = Chlorobenzene-d5

DCBd4 = 1,4-Dichlorobenzene-d4

Area Limit $=50 \%-200 \%$ of internal standard area

$\mathrm{RT}$ Limit $= \pm 0.1667$ minutes of internal standard RT

\# Column used to flag values outside QC limits

FORM VIII SOM02.2/VOA_TR 
FORM VIII

GC/MS VOA INTERNAL STANDARD AREA AND RETENTION TIME SUMMARY

Lab Name: TestAmerica Burlington

SDG No.: 200-38792-1

Sample No.: CCVIS 200-117296/6

Instrument ID: CHD.i

Lab File ID (Standard): 25366_06.D

Calibration ID: 37252
Job No.: 200-38792-1

Date Analyzed: 06/06/2017 15:17

GC Column: DB-624

ID $: 0.2(\mathrm{~mm})$

Heated Purge: (Y/N) N

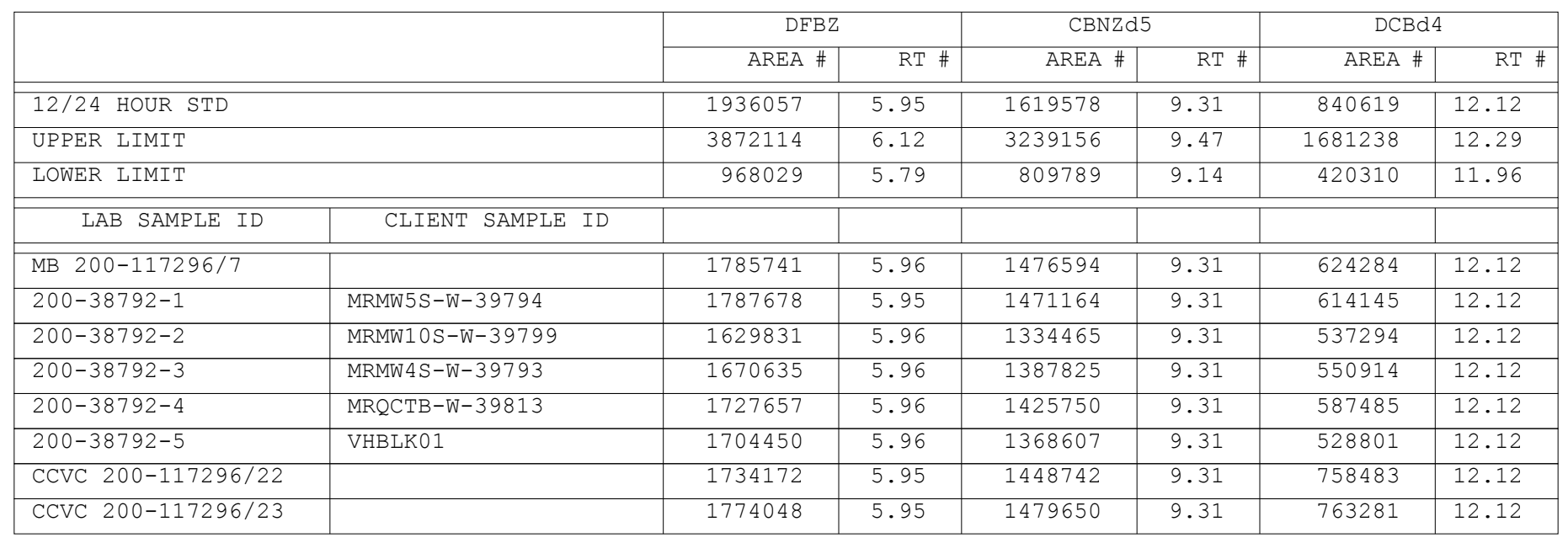

DFBZ = 1,4-Difluorobenzene

CBNZd5 = Chlorobenzene-d5

DCBd4 = 1,4-Dichlorobenzene-d4

Area Limit $=50 \%-200 \%$ of internal standard area

$\mathrm{RT}$ Limit $= \pm 0.1667$ minutes of internal standard RT

\# Column used to flag values outside QC limits

FORM VIII SOM02.2/VOA_TR 
FORM VIII

GC/MS VOA INTERNAL STANDARD AREA AND RETENTION TIME SUMMARY

Lab Name: TestAmerica Burlington

Job No. : 200-38792-1

SDG No.: 200-38792-1

Sample No.: CCVIS 200-117409/5

Instrument ID: CHD.i

Lab File ID (Standard): 25405_05.D

Date Analyzed: 06/08/2017 15:53

Calibration ID: 37252

GC Column: DB-624

Heated Purge: (Y/N) N

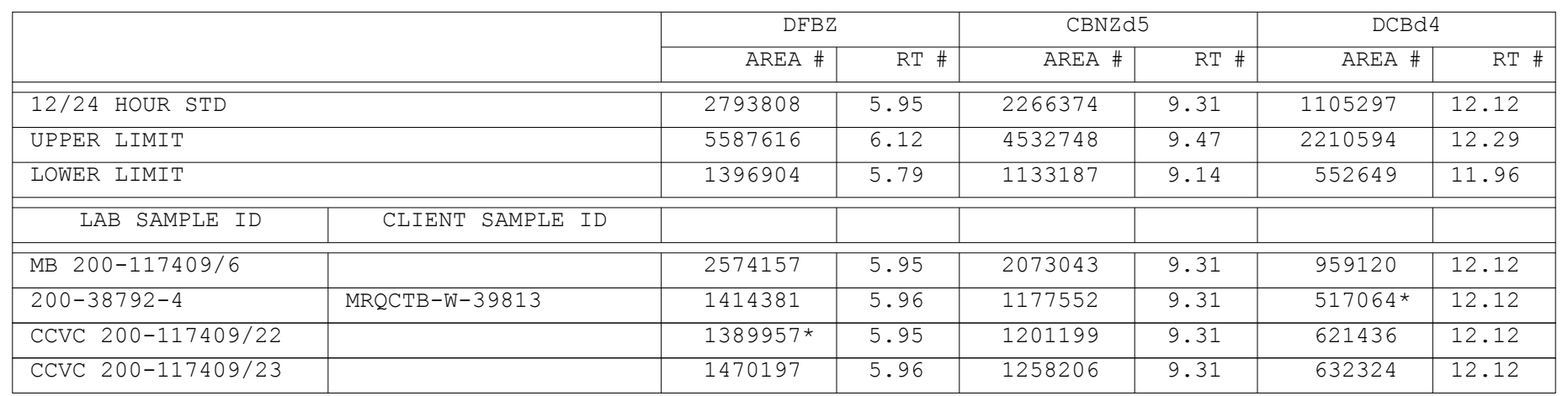

DFBZ = 1,4-Difluorobenzene

CBNZd5 = Chlorobenzene-d5

DCBd4 = 1,4-Dichlorobenzene-d4

Area Limit $=50 \%-200 \%$ of internal standard area

$\mathrm{RT}$ Limit $= \pm 0.1667$ minutes of internal standard RT

\# Column used to flag values outside QC limits

FORM VIII SOM02.2/VOA_TR 
FORM I

GC/MS VOA ORGANICS ANALYSIS DATA SHEET

Lab Name: TestAmerica Burlington

SDG No.: 200-38792-1

Client Sample ID: MRMW5S-W-39794

Matrix: Water

Analysis Method: SOM02.2/VOA_Tr

Sample wt/vol: 25 (mL)

Soil Aliquot Vol:

Soil Extract Vol.:

응 Moisture:

Analysis Batch No.: 117296
Job No. : 200-38792-1

Lab Sample ID: 200-38792-1

Lab File ID: 25366_08.D

Date Collected: 06/01/2017 15:00

Date Analyzed: 06/06/2017 17:59

Dilution Factor: 1

GC Column: DB-624

ID: $0.2(\mathrm{~mm})$

Level: (low/med) Trace

Units: ug/L

\begin{tabular}{|c|c|c|c|c|}
\hline CAS NO. & COMPOUND NAME & RESULT & $Q$ & $\mathrm{RL}$ \\
\hline $75-71-8$ & Dichlorodifluoromethane & 0.50 & $\mathrm{U}$ & 0.50 \\
\hline $74-87-3$ & Chloromethane & 0.50 & $\mathrm{U}$ & 0.50 \\
\hline $75-01-4$ & Vinyl chloride & 0.50 & $\mathrm{U}$ & 0.50 \\
\hline $74-83-9$ & Bromomethane & 0.50 & $\mathrm{U}$ & 0.50 \\
\hline $75-00-3$ & Chloroethane & 0.50 & $\mathrm{U}$ & 0.50 \\
\hline $75-69-4$ & Trichlorofluoromethane & 0.50 & $\mathrm{U}$ & 0.50 \\
\hline $75-35-4$ & 1,1-Dichloroethene & 0.50 & $\mathrm{U}$ & 0.50 \\
\hline $76-13-1$ & $\begin{array}{l}\text { 1, 1,2-Trichloro-1,2,2-trifluoroethan } \\
\text { e }\end{array}$ & 0.50 & $\mathrm{U}$ & 0.50 \\
\hline $67-64-1$ & Acetone & 5.0 & $\mathrm{U}$ & 5.0 \\
\hline $75-15-0$ & Carbon disulfide & 0.15 & $\mathrm{~J} B$ & 0.50 \\
\hline $79-20-9$ & Methyl acetate & 0.50 & $\mathrm{U}$ & 0.50 \\
\hline $75-09-2$ & Methylene Chloride & 0.50 & $\mathrm{U}$ & 0.50 \\
\hline $156-60-5$ & trans-1,2-Dichloroethene & 0.50 & $\mathrm{U}$ & 0.50 \\
\hline $1634-04-4$ & Methyl tert-butyl ether & 0.50 & $\mathrm{U}$ & 0.50 \\
\hline $75-34-3$ & 1,1-Dichloroethane & 0.50 & $\mathrm{U}$ & 0.50 \\
\hline $156-59-2$ & cis-1,2-Dichloroethene & 0.50 & $\mathrm{U}$ & 0.50 \\
\hline $78-93-3$ & 2-Butanone & 5.0 & $\mathrm{U}$ & 5.0 \\
\hline $74-97-5$ & Bromochloromethane & 0.50 & $\mathrm{U}$ & 0.50 \\
\hline $67-66-3$ & Chloroform & 0.50 & $\mathrm{U}$ & 0.50 \\
\hline $71-55-6$ & $1,1,1$-Trichloroethane & 0.50 & $\mathrm{U}$ & 0.50 \\
\hline $110-82-7$ & Cyclohexane & 0.50 & $\mathrm{U}$ & 0.50 \\
\hline $56-23-5$ & Carbon tetrachloride & 0.50 & $\mathrm{U}$ & 0.50 \\
\hline $71-43-2$ & Benzene & 0.50 & $\mathrm{U}$ & 0.50 \\
\hline $107-06-2$ & 1,2-Dichloroethane & 0.50 & $\mathrm{U}$ & 0.50 \\
\hline $79-01-6$ & Trichloroethene & 0.50 & $\mathrm{U}$ & 0.50 \\
\hline $108-87-2$ & Methylcyclohexane & 0.50 & $\mathrm{U}$ & 0.50 \\
\hline $78-87-5$ & 1,2-Dichloropropane & 0.50 & $\mathrm{U}$ & 0.50 \\
\hline $75-27-4$ & Bromodichloromethane & 0.50 & $\mathrm{U}$ & 0.50 \\
\hline $10061-01-5$ & cis-1,3-Dichloropropene & 0.50 & $\mathrm{U}$ & 0.50 \\
\hline $108-10-1$ & 4-Methyl-2-pentanone & 5.0 & $\mathrm{U}$ & 5.0 \\
\hline $108-88-3$ & Toluene & 0.50 & $\mathrm{U}$ & 0.50 \\
\hline $10061-02-6$ & trans-1,3-Dichloropropene & 0.50 & $\mathrm{U}$ & 0.50 \\
\hline $79-00-5$ & 1,1,2-Trichloroethane & 0.50 & $\mathrm{U}$ & 0.50 \\
\hline $127-18-4$ & Tetrachloroethene & 0.50 & $\mathrm{U}$ & 0.50 \\
\hline $591-78-6$ & 2-Hexanone & 5.0 & $\mathrm{U}$ & 5.0 \\
\hline
\end{tabular}


FORM I

GC/MS VOA ORGANICS ANALYSIS DATA SHEET

Lab Name: TestAmerica Burlington

SDG No.: 200-38792-1

Client Sample ID: MRMW5S-W-39794

Matrix: Water

Analysis Method: SOM02.2/VOA_Tr

Sample wt/vol: 25 (mL)

Soil Aliquot Vol:

Soil Extract Vol.:

응 Moisture:

Analysis Batch No.: 117296
Job No. : 200-38792-1

Lab Sample ID: 200-38792-1

Lab File ID: 25366_08.D

Date Collected: 06/01/2017 15:00

Date Analyzed: 06/06/2017 17:59

Dilution Factor: 1

GC Column: DB-624

ID: $0.2(\mathrm{~mm})$

Level: (low/med) Trace

Units: ug/L

\begin{tabular}{|c|c|c|c|c|}
\hline CAS NO. & COMPOUND NAME & RESULT & $Q$ & RL \\
\hline $124-48-1$ & Dibromochloromethane & 0.50 & $\mathrm{U}$ & 0.50 \\
\hline $106-93-4$ & 1,2-Dibromoethane & 0.50 & $\mathrm{U}$ & 0.50 \\
\hline $108-90-7$ & Chlorobenzene & 0.50 & $\mathrm{U}$ & 0.50 \\
\hline $100-41-4$ & Ethylbenzene & 0.50 & $\mathrm{U}$ & 0.50 \\
\hline $95-47-6$ & o-Xylene & 0.50 & $\mathrm{U}$ & 0.50 \\
\hline $179601-23-1$ & m,p-Xylene & 0.50 & $\mathrm{U}$ & 0.50 \\
\hline $100-42-5$ & Styrene & 0.50 & $\mathrm{U}$ & 0.50 \\
\hline $75-25-2$ & Bromoform & 0.50 & $\mathrm{U}$ & 0.50 \\
\hline $98-82-8$ & Isopropylbenzene & 0.50 & $\mathrm{U}$ & 0.50 \\
\hline $79-34-5$ & $1,1,2,2$-Tetrachloroethane & 0.50 & $\mathrm{U}$ & 0.50 \\
\hline $541-73-1$ & 1,3-Dichlorobenzene & 0.50 & $\mathrm{U}$ & 0.50 \\
\hline $106-46-7$ & 1,4-Dichlorobenzene & 0.50 & $\mathrm{U}$ & 0.50 \\
\hline $95-50-1$ & 1,2-Dichlorobenzene & 0.50 & $\mathrm{U}$ & 0.50 \\
\hline $96-12-8$ & 1,2-Dibromo-3-chloropropane & 0.50 & $\mathrm{U}$ & 0.50 \\
\hline $120-82-1$ & $1,2,4$-Trichlorobenzene & 0.068 & $\mathrm{~J} B$ & 0.50 \\
\hline $87-61-6$ & $1,2,3$-Trichlorobenzene & 0.068 & & 0.50 \\
\hline
\end{tabular}

\begin{tabular}{|c|c|c|c|c|}
\hline CAS NO. & SURROGATE & $\because \mathrm{REC}$ & $Q$ & LIMITS \\
\hline $6745-35-3$ & Vinyl chloride-d3 & 106 & & $40-130$ \\
\hline $19199-91-8$ & Chloroethane-d5 & 124 & & $65-130$ \\
\hline $22280-73-5$ & 1,1-Dichloroethene-d2 & 100 & & $60-125$ \\
\hline $24313-50-6$ & 2-Butanone-d5 & 150 & * & $40-130$ \\
\hline $865-49-6$ & Chloroform-d & 121 & & $70-125$ \\
\hline $17060-07-0$ & 1,2-Dichloroethane-d4 & 137 & * & $70-130$ \\
\hline $1076-43-3$ & Benzene-d6 & 118 & & $70-125$ \\
\hline $93952-08-0$ & 1,2-Dichloropropane-d6 & 95 & & $60-140$ \\
\hline $2037-26-5$ & Toluene-d8 & 117 & & $70-130$ \\
\hline $93951-86-1$ & trans-1,3-Dichloropropene-d4 & 110 & & $55-130$ \\
\hline $4840-82-8$ & 2-Hexanone-d5 & 145 & * & $45-130$ \\
\hline $33685-54-0$ & $1,1,2,2$-Tetrachloroethane-d2 & 110 & & $65-120$ \\
\hline $2199-69-1$ & 1,2 -Dichlorobenzene-d4 & 116 & & $80-120$ \\
\hline
\end{tabular}


FORM I

GC/MS VOA ORGANICS ANALYSIS DATA SHEET

TENTATIVELY IDENTIFIED COMPOUNDS

Lab Name: TestAmerica Burlington

SDG No.: 200-38792-1

Client Sample ID: MRMW5S-W-39794

Matrix: Water

Analysis Method: SOM02.2/VOA_Tr

Sample wt/vol: $25(\mathrm{~mL})$

Soil Aliquot Vol:

Soil Extract Vol.:

\% Moisture:

Analysis Batch No.: 117296

Number TICS Found: 4
Job No.: 200-38792-1

Lab Sample ID: 200-38792-1

Lab File ID: 25366 08.D

Date Collected: 06/01/2017 15:00

Date Analyzed: 06/06/2017 17:59

Dilution Factor: 1

GC Column: DB-624

ID $: 0.2(\mathrm{~mm})$

Level: (low/med) Trace

Units: ug/L

TIC Result Total: 6.11

\begin{tabular}{|c|c|c|c|c|c|}
\hline CAS NO. & COMPOUND NAME & $\mathrm{RT}$ & RESULT & $Q$ & $\begin{array}{c}\text { MATCH } \\
\text { QUALITY }\end{array}$ \\
\hline $78-79-5$ & Isoprene & 2.83 & 0.57 & $\mathrm{~J} \mathrm{~N}$ & $95 \%$ \\
\hline & Unknown & 7.28 & 3.1 & $\mathrm{~J} B \mathrm{X}$ & \\
\hline $541-05-9$ & Cyclotrisiloxane, hexamethyl- & 8.18 & 0.54 & $\mathrm{~J} \mathrm{~N}$ & $91 \%$ \\
\hline $556-67-2$ & Cyclotetrasiloxane, octamethyl- & 11.01 & 1.9 & $\mathrm{~J} \mathrm{~N}$ & $91 \%$ \\
\hline
\end{tabular}


FORM I

GC/MS VOA ORGANICS ANALYSIS DATA SHEET

TARGETED TENATIVELY IDENTIFIED COMPOUNDS

Lab Name: TestAmerica Burlington

SDG No.: 200-38792-1

Client Sample ID: MRMW5S-W-39794

Matrix: Water

Analysis Method: SOM02.2/VOA_Tr

Sample wt/vol: 25 (mL)

Soil Aliquot Vol:

Soil Extract Vol.:

․ Moisture:

Analysis Batch No.: 117296
Job No.: 200-38792-1

Lab Sample ID: 200-38792-1

Lab File ID: $2536608 . \mathrm{D}$

Date Collected: 06/01/2017 15:00

Date Analyzed: 06/06/2017 17:59

Dilution Factor: 1

GC Column: DB-624

ID $: 0.2(\mathrm{~mm})$

Level: (low/med) Trace

Units: ug/L

\begin{tabular}{|c|c|c|c|c|}
\hline CAS NO. & COMPOUND NAME & RT & RESULT & Q \\
\hline QUALITY
\end{tabular}


FORM I

GC/MS VOA ORGANICS ANALYSIS DATA SHEET

Lab Name: TestAmerica Burlington

SDG No.: 200-38792-1

Client Sample ID: MRMW10S-W-39799

Matrix: Water

Analysis Method: SOM02.2/VOA_Tr

Sample wt/vol: 25 (mL)

Soil Aliquot Vol:

Soil Extract Vol.:

응 Moisture:

Analysis Batch No.: 117296
Job No. : 200-38792-1

Lab Sample ID: 200-38792-2

Lab File ID: 25366_09.D

Date Collected: 06/01/2017 17:15

Date Analyzed: 06/06/2017 18:25

Dilution Factor: 1

GC Column: DB-624

ID: $0.2(\mathrm{~mm})$

Level: (low/med) Trace

Units: ug/L

\begin{tabular}{|c|c|c|c|c|}
\hline CAS NO. & COMPOUND NAME & RESULT & $Q$ & $\mathrm{RL}$ \\
\hline $75-71-8$ & Dichlorodifluoromethane & 0.50 & $\mathrm{U}$ & 0.50 \\
\hline $74-87-3$ & Chloromethane & 0.50 & $\mathrm{U}$ & 0.50 \\
\hline $75-01-4$ & Vinyl chloride & 0.50 & $\mathrm{U}$ & 0.50 \\
\hline $74-83-9$ & Bromomethane & 0.50 & $\mathrm{U}$ & 0.50 \\
\hline $75-00-3$ & Chloroethane & 0.50 & $\mathrm{U}$ & 0.50 \\
\hline $75-69-4$ & Trichlorofluoromethane & 0.50 & $\mathrm{U}$ & 0.50 \\
\hline $75-35-4$ & 1,1-Dichloroethene & 0.50 & $\mathrm{U}$ & 0.50 \\
\hline $76-13-1$ & $\begin{array}{l}\text { 1, 1,2-Trichloro-1,2,2-trifluoroethan } \\
\text { e }\end{array}$ & 0.50 & $\mathrm{U}$ & 0.50 \\
\hline $67-64-1$ & Acetone & 5.0 & $\mathrm{U}$ & 5.0 \\
\hline $75-15-0$ & Carbon disulfide & 0.11 & $\mathrm{~J} B$ & 0.50 \\
\hline $79-20-9$ & Methyl acetate & 0.50 & $\mathrm{U}$ & 0.50 \\
\hline $75-09-2$ & Methylene Chloride & 0.50 & $\mathrm{U}$ & 0.50 \\
\hline $156-60-5$ & trans-1,2-Dichloroethene & 0.50 & $\mathrm{U}$ & 0.50 \\
\hline $1634-04-4$ & Methyl tert-butyl ether & 0.50 & $\mathrm{U}$ & 0.50 \\
\hline $75-34-3$ & 1,1-Dichloroethane & 0.50 & $\mathrm{U}$ & 0.50 \\
\hline $156-59-2$ & cis-1,2-Dichloroethene & 0.50 & $\mathrm{U}$ & 0.50 \\
\hline $78-93-3$ & 2-Butanone & 5.0 & $\mathrm{U}$ & 5.0 \\
\hline $74-97-5$ & Bromochloromethane & 0.50 & $\mathrm{U}$ & 0.50 \\
\hline $67-66-3$ & Chloroform & 2.6 & & 0.50 \\
\hline $71-55-6$ & $1,1,1$-Trichloroethane & 0.50 & $\mathrm{U}$ & 0.50 \\
\hline $110-82-7$ & Cyclohexane & 0.50 & $\mathrm{U}$ & 0.50 \\
\hline $56-23-5$ & Carbon tetrachloride & 0.31 & $\mathrm{~J}$ & 0.50 \\
\hline $71-43-2$ & Benzene & 0.50 & $\mathrm{U}$ & 0.50 \\
\hline $107-06-2$ & 1,2-Dichloroethane & 0.50 & $\mathrm{U}$ & 0.50 \\
\hline $79-01-6$ & Trichloroethene & 0.50 & $\mathrm{U}$ & 0.50 \\
\hline $108-87-2$ & Methylcyclohexane & 0.50 & $\mathrm{U}$ & 0.50 \\
\hline $78-87-5$ & 1,2-Dichloropropane & 0.50 & $\mathrm{U}$ & 0.50 \\
\hline $75-27-4$ & Bromodichloromethane & 0.50 & $\mathrm{U}$ & 0.50 \\
\hline $10061-01-5$ & cis-1,3-Dichloropropene & 0.50 & $\mathrm{U}$ & 0.50 \\
\hline $108-10-1$ & 4-Methyl-2-pentanone & 5.0 & $\mathrm{U}$ & 5.0 \\
\hline $108-88-3$ & Toluene & 0.50 & $\mathrm{U}$ & 0.50 \\
\hline $10061-02-6$ & trans-1,3-Dichloropropene & 0.50 & $\mathrm{U}$ & 0.50 \\
\hline $79-00-5$ & 1,1,2-Trichloroethane & 0.50 & $\mathrm{U}$ & 0.50 \\
\hline $127-18-4$ & Tetrachloroethene & 0.50 & $\mathrm{U}$ & 0.50 \\
\hline $591-78-6$ & 2-Hexanone & 5.0 & $\mathrm{U}$ & 5.0 \\
\hline
\end{tabular}

FORM I SOM02.2/VOA_Tr 
FORM I

GC/MS VOA ORGANICS ANALYSIS DATA SHEET

Lab Name: TestAmerica Burlington

SDG No.: 200-38792-1

Client Sample ID: MRMW10S-W-39799

Matrix: Water

Analysis Method: SOM02.2/VOA_Tr

Sample wt/vol: 25 (mL)

Soil Aliquot Vol:

Soil Extract Vol.:

응 Moisture:

Analysis Batch No.: 117296
Job No. : 200-38792-1

Lab Sample ID: 200-38792-2

Lab File ID: 25366_09.D

Date Collected: 06/01/2017 17:15

Date Analyzed: 06/06/2017 18:25

Dilution Factor: 1

GC Column: DB-624

ID: $0.2(\mathrm{~mm})$

Level: (low/med) Trace

Units: ug/L

\begin{tabular}{|c|c|c|c|c|}
\hline CAS NO. & COMPOUND NAME & RESULT & $Q$ & RL \\
\hline $124-48-1$ & Dibromochloromethane & 0.50 & $\mathrm{U}$ & 0.50 \\
\hline $106-93-4$ & 1,2-Dibromoethane & 0.50 & $\mathrm{U}$ & 0.50 \\
\hline $108-90-7$ & Chlorobenzene & 0.50 & $\mathrm{U}$ & 0.50 \\
\hline $100-41-4$ & Ethylbenzene & 0.50 & $\mathrm{U}$ & 0.50 \\
\hline $95-47-6$ & o-Xylene & 0.50 & $\mathrm{U}$ & 0.50 \\
\hline $179601-23-1$ & m,p-Xylene & 0.50 & $\mathrm{U}$ & 0.50 \\
\hline $100-42-5$ & Styrene & 0.50 & $\mathrm{U}$ & 0.50 \\
\hline $75-25-2$ & Bromoform & 0.50 & $\mathrm{U}$ & 0.50 \\
\hline $98-82-8$ & Isopropylbenzene & 0.50 & $\mathrm{U}$ & 0.50 \\
\hline $79-34-5$ & $1,1,2,2$-Tetrachloroethane & 0.50 & $\mathrm{U}$ & 0.50 \\
\hline $541-73-1$ & 1,3-Dichlorobenzene & 0.50 & $\mathrm{U}$ & 0.50 \\
\hline $106-46-7$ & 1,4-Dichlorobenzene & 0.50 & $\mathrm{U}$ & 0.50 \\
\hline $95-50-1$ & 1,2-Dichlorobenzene & 0.50 & $\mathrm{U}$ & 0.50 \\
\hline $96-12-8$ & 1,2-Dibromo-3-chloropropane & 0.50 & $\mathrm{U}$ & 0.50 \\
\hline $120-82-1$ & $1,2,4$-Trichlorobenzene & 0.50 & $\mathrm{U}$ & 0.50 \\
\hline $87-61-6$ & $1,2,3$-Trichlorobenzene & 0.50 & $\mathrm{U}$ & 0.50 \\
\hline
\end{tabular}

\begin{tabular}{|c|c|c|c|c|}
\hline CAS NO. & SURROGATE & $\because \mathrm{REC}$ & $Q$ & LIMITS \\
\hline $6745-35-3$ & Vinyl chloride-d3 & 103 & & $40-130$ \\
\hline $19199-91-8$ & Chloroethane-d5 & 122 & & $65-130$ \\
\hline $22280-73-5$ & 1,1-Dichloroethene-d2 & 96 & & $60-125$ \\
\hline $24313-50-6$ & 2-Butanone-d5 & 149 & * & $40-130$ \\
\hline $865-49-6$ & Chloroform-d & 124 & & $70-125$ \\
\hline $17060-07-0$ & 1,2-Dichloroethane-d4 & 139 & * & $70-130$ \\
\hline $1076-43-3$ & Benzene-d6 & 117 & & $70-125$ \\
\hline $93952-08-0$ & 1,2-Dichloropropane-d6 & 96 & & $60-140$ \\
\hline $2037-26-5$ & Toluene-d8 & 117 & & $70-130$ \\
\hline $93951-86-1$ & trans-1,3-Dichloropropene-d4 & 112 & & $55-130$ \\
\hline $4840-82-8$ & 2-Hexanone-d5 & 148 & * & $45-130$ \\
\hline $33685-54-0$ & $1,1,2,2$-Tetrachloroethane-d2 & 110 & & $65-120$ \\
\hline $2199-69-1$ & 1,2 -Dichlorobenzene-d4 & 119 & & $80-120$ \\
\hline
\end{tabular}


FORM I

GC/MS VOA ORGANICS ANALYSIS DATA SHEET

TENTATIVELY IDENTIFIED COMPOUNDS

Lab Name: TestAmerica Burlington

SDG No.: 200-38792-1

Client Sample ID: MRMW10S-W-39799

Matrix: Water

Analysis Method: SOM02.2/VOA_Tr

Sample wt/vol: $25(\mathrm{~mL})$

Soil Aliquot Vol:

Soil Extract Vol.:

\% Moisture:

Analysis Batch No.: 117296

Number TICs Found: 1

\begin{tabular}{|c|c|r|r|r|}
\hline CAS NO. & COMPOUND NAME & RT & RESULT & Q \\
\hline QUALITY \\
\hline \hline
\end{tabular}

Job No.: 200-38792-1

Lab Sample ID: 200-38792-2

Lab File ID: 25366_09.D

Date Collected: 06/01/2017 17:15

Date Analyzed: 06/06/2017 18:25

Dilution Factor: 1

GC Column: DB-624

ID $: 0.2(\mathrm{~mm})$

Level: (low/med) Trace

Units: ug/L

TIC Result Total: 3 
FORM I

GC/MS VOA ORGANICS ANALYSIS DATA SHEET

TARGETED TENATIVELY IDENTIFIED COMPOUNDS

Lab Name: TestAmerica Burlington

SDG No.: 200-38792-1

Client Sample ID: MRMW10S-W-39799

Matrix: Water

Analysis Method: SOM02.2/VOA_Tr

Sample wt/vol: 25 (mL)

Soil Aliquot Vol:

Soil Extract Vol.:

․ Moisture:

Analysis Batch No.: 117296
Job No.: 200-38792-1

Lab Sample ID: 200-38792-2

Lab File ID: 25366 09.D

Date Collected: 06/01/2017 17:15

Date Analyzed: 06/06/2017 18:25

Dilution Factor: 1

GC Column: DB-624

ID $: 0.2(\mathrm{~mm})$

Level: (low/med) Trace

Units: ug/L

\begin{tabular}{|c|c|c|c|c|}
\hline CAS NO. & COMPOUND NAME & RT & RESULT & Q \\
\hline QUALITY
\end{tabular}


FORM I

GC/MS VOA ORGANICS ANALYSIS DATA SHEET

Lab Name: TestAmerica Burlington

SDG No.: 200-38792-1

Client Sample ID: MRMW4S-W-39793

Matrix: Water

Analysis Method: SOM02.2/VOA_Tr

Sample wt/vol: 25 (mL)

Soil Aliquot Vol:

Soil Extract Vol.:

응 Moisture:

Analysis Batch No.: 117296
Job No. : 200-38792-1

Lab Sample ID: 200-38792-3

Lab File ID: 25366_10.D

Date Collected: 06/02/2017 10:50

Date Analyzed: 06/06/2017 18:51

Dilution Factor: 1

GC Column: DB-624

ID: $0.2(\mathrm{~mm})$

Level: (low/med) Trace

Units: ug/L

\begin{tabular}{|c|c|c|c|c|}
\hline CAS NO. & COMPOUND NAME & RESULT & $Q$ & RL \\
\hline $75-71-8$ & Dichlorodifluoromethane & 0.50 & $\mathrm{U}$ & 0.50 \\
\hline $74-87-3$ & Chloromethane & 0.50 & $\mathrm{U}$ & 0.50 \\
\hline $75-01-4$ & Vinyl chloride & 0.50 & $\mathrm{U}$ & 0.50 \\
\hline $74-83-9$ & Bromomethane & 0.50 & $\mathrm{U}$ & 0.50 \\
\hline $75-00-3$ & Chloroethane & 0.50 & $\mathrm{U}$ & 0.50 \\
\hline $75-69-4$ & Trichlorofluoromethane & 0.50 & $\mathrm{U}$ & 0.50 \\
\hline $75-35-4$ & 1,1-Dichloroethene & 0.50 & $\mathrm{U}$ & 0.50 \\
\hline $76-13-1$ & $\begin{array}{l}\text { 1, 1,2-Trichloro-1,2,2-trifluoroethan } \\
\text { e }\end{array}$ & 0.50 & $\mathrm{U}$ & 0.50 \\
\hline $67-64-1$ & Acetone & 5.0 & $\mathrm{U}$ & 5.0 \\
\hline $75-15-0$ & Carbon disulfide & 0.11 & $\mathrm{~J} B$ & 0.50 \\
\hline $79-20-9$ & Methyl acetate & 0.50 & $\mathrm{U}$ & 0.50 \\
\hline $75-09-2$ & Methylene Chloride & 0.50 & $\mathrm{U}$ & 0.50 \\
\hline $156-60-5$ & trans-1,2-Dichloroethene & 0.50 & $\mathrm{U}$ & 0.50 \\
\hline $1634-04-4$ & Methyl tert-butyl ether & 0.50 & $\mathrm{U}$ & 0.50 \\
\hline $75-34-3$ & 1,1-Dichloroethane & 0.50 & $\mathrm{U}$ & 0.50 \\
\hline $156-59-2$ & cis-1,2-Dichloroethene & 0.50 & $\mathrm{U}$ & 0.50 \\
\hline $78-93-3$ & 2-Butanone & 5.0 & $\mathrm{U}$ & 5.0 \\
\hline $74-97-5$ & Bromochloromethane & 0.50 & $\mathrm{U}$ & 0.50 \\
\hline $67-66-3$ & Chloroform & 0.50 & $\mathrm{U}$ & 0.50 \\
\hline $71-55-6$ & $1,1,1$-Trichloroethane & 0.50 & $\mathrm{U}$ & 0.50 \\
\hline $110-82-7$ & Cyclohexane & 0.50 & $\mathrm{U}$ & 0.50 \\
\hline $56-23-5$ & Carbon tetrachloride & 0.11 & $\mathrm{~J}$ & 0.50 \\
\hline $71-43-2$ & Benzene & 0.50 & $\mathrm{U}$ & 0.50 \\
\hline $107-06-2$ & 1,2-Dichloroethane & 0.50 & $\mathrm{U}$ & 0.50 \\
\hline $79-01-6$ & Trichloroethene & 0.50 & $\mathrm{U}$ & 0.50 \\
\hline $108-87-2$ & Methylcyclohexane & 0.50 & $\mathrm{U}$ & 0.50 \\
\hline $78-87-5$ & 1,2-Dichloropropane & 0.50 & $\mathrm{U}$ & 0.50 \\
\hline $75-27-4$ & Bromodichloromethane & 0.50 & $\mathrm{U}$ & 0.50 \\
\hline $10061-01-5$ & cis-1,3-Dichloropropene & 0.50 & $\mathrm{U}$ & 0.50 \\
\hline $108-10-1$ & 4-Methyl-2-pentanone & 5.0 & $\mathrm{U}$ & 5.0 \\
\hline $108-88-3$ & Toluene & 0.50 & $\mathrm{U}$ & 0.50 \\
\hline $10061-02-6$ & trans-1,3-Dichloropropene & 0.50 & $\mathrm{U}$ & 0.50 \\
\hline $79-00-5$ & $1,1,2$-Trichloroethane & 0.50 & $\mathrm{U}$ & 0.50 \\
\hline $127-18-4$ & Tetrachloroethene & 0.50 & $\mathrm{U}$ & 0.50 \\
\hline $591-78-6$ & 2-Hexanone & 5.0 & $\mathrm{U}$ & 5.0 \\
\hline
\end{tabular}


FORM I

GC/MS VOA ORGANICS ANALYSIS DATA SHEET

Lab Name: TestAmerica Burlington

SDG No.: 200-38792-1

Client Sample ID: MRMW4S-W-39793

Matrix: Water

Analysis Method: SOM02.2/VOA_Tr

Sample wt/vol: 25 (mL)

Soil Aliquot Vol:

Soil Extract Vol.:

응 Moisture:

Analysis Batch No.: 117296
Job No. : 200-38792-1

Lab Sample ID: 200-38792-3

Lab File ID: 25366 10.D

Date Collected: 06/02/2017 10:50

Date Analyzed: 06/06/2017 18:51

Dilution Factor: 1

GC Column: DB-624

ID: $0.2(\mathrm{~mm})$

Level: (low/med) Trace

Units: ug/L

\begin{tabular}{|c|c|c|c|c|}
\hline CAS NO. & COMPOUND NAME & RESULT & $Q$ & RL \\
\hline $124-48-1$ & Dibromochloromethane & 0.50 & $\mathrm{U}$ & 0.50 \\
\hline $106-93-4$ & 1,2-Dibromoethane & 0.50 & $\mathrm{U}$ & 0.50 \\
\hline $108-90-7$ & Chlorobenzene & 0.50 & $\mathrm{U}$ & 0.50 \\
\hline $100-41-4$ & Ethylbenzene & 0.50 & $\mathrm{U}$ & 0.50 \\
\hline $95-47-6$ & o-Xylene & 0.50 & $\mathrm{U}$ & 0.50 \\
\hline $179601-23-1$ & m,p-Xylene & 0.50 & $\mathrm{U}$ & 0.50 \\
\hline $100-42-5$ & Styrene & 0.50 & $\mathrm{U}$ & 0.50 \\
\hline $75-25-2$ & Bromoform & 0.50 & $\mathrm{U}$ & 0.50 \\
\hline $98-82-8$ & Isopropylbenzene & 0.50 & $\mathrm{U}$ & 0.50 \\
\hline $79-34-5$ & $1,1,2,2$-Tetrachloroethane & 0.50 & $\mathrm{U}$ & 0.50 \\
\hline $541-73-1$ & 1,3-Dichlorobenzene & 0.50 & $\mathrm{U}$ & 0.50 \\
\hline $106-46-7$ & 1,4-Dichlorobenzene & 0.50 & $\mathrm{U}$ & 0.50 \\
\hline $95-50-1$ & 1,2-Dichlorobenzene & 0.50 & $\mathrm{U}$ & 0.50 \\
\hline $96-12-8$ & 1,2-Dibromo-3-chloropropane & 0.50 & $\mathrm{U}$ & 0.50 \\
\hline $120-82-1$ & $1,2,4$-Trichlorobenzene & 0.50 & $\mathrm{U}$ & 0.50 \\
\hline $87-61-6$ & $1,2,3$-Trichlorobenzene & 0.50 & $\mathrm{U}$ & 0.50 \\
\hline
\end{tabular}

\begin{tabular}{|c|c|c|c|c|}
\hline CAS NO. & SURROGATE & $\because \mathrm{REC}$ & $Q$ & LIMITS \\
\hline $6745-35-3$ & Vinyl chloride-d3 & 103 & & $40-130$ \\
\hline $19199-91-8$ & Chloroethane-d5 & 121 & & $65-130$ \\
\hline $22280-73-5$ & 1,1-Dichloroethene-d2 & 94 & & $60-125$ \\
\hline $24313-50-6$ & 2-Butanone-d5 & 152 & * & $40-130$ \\
\hline $865-49-6$ & Chloroform-d & 123 & & $70-125$ \\
\hline $17060-07-0$ & 1,2-Dichloroethane-d4 & 139 & * & $70-130$ \\
\hline $1076-43-3$ & Benzene-d6 & 117 & & $70-125$ \\
\hline $93952-08-0$ & 1,2-Dichloropropane-d6 & 95 & & $60-140$ \\
\hline $2037-26-5$ & Toluene-d8 & 116 & & $70-130$ \\
\hline $93951-86-1$ & trans-1,3-Dichloropropene-d4 & 111 & & $55-130$ \\
\hline $4840-82-8$ & 2-Hexanone-d5 & 150 & * & $45-130$ \\
\hline $33685-54-0$ & $1,1,2,2$-Tetrachloroethane-d2 & 113 & & $65-120$ \\
\hline $2199-69-1$ & 1,2 -Dichlorobenzene-d4 & 120 & & $80-120$ \\
\hline
\end{tabular}


FORM I

GC/MS VOA ORGANICS ANALYSIS DATA SHEET

TENTATIVELY IDENTIFIED COMPOUNDS

Lab Name: TestAmerica Burlington

SDG No.: 200-38792-1

Client Sample ID: MRMW4S-W-39793

Matrix: Water

Analysis Method: SOM02.2/VOA_Tr

Sample wt/vol: $25(\mathrm{~mL})$

Soil Aliquot Vol:

Soil Extract Vol.:

\% Moisture:

Analysis Batch No.: 117296

Number TICs Found: 2

\begin{tabular}{|c|l|r|r|r|r|}
\hline CAS NO. & COMPOUND NAME & RT & RESULT & Q & $\begin{array}{c}\text { MATCH } \\
\text { QUAITY }\end{array}$ \\
\hline \hline $78-79-5$ & Isoprene & 2.83 & 0.51 & $\mathrm{~J} \mathrm{~N}$ & $92 \%$ \\
\hline & Unknown & 7.28 & 3.0 & $\mathrm{~J} \mathrm{~B}$ & \\
\hline
\end{tabular}

Job No.: 200-38792-1

Lab Sample ID: 200-38792-3

Lab File ID: 25366_10.D

Date Collected: 06/02/2017 10:50

Date Analyzed: 06/06/2017 18:51

Dilution Factor: 1

GC Column: DB-624

ID $: 0.2(\mathrm{~mm})$

Level: (low/med) Trace

Units: ug/L

TIC Result Total: 3.51 
FORM I

GC/MS VOA ORGANICS ANALYSIS DATA SHEET

TARGETED TENATIVELY IDENTIFIED COMPOUNDS

Lab Name: TestAmerica Burlington

Job No.: 200-38792-1

SDG No.: 200-38792-1

Client Sample ID: MRMW4S-W-39793

Matrix: Water

Analysis Method: SOM02.2/VOA_Tr

Sample wt/vol: 25 (mL)

Soil Aliquot Vol:

Lab Sample ID: 200-38792-3

Lab File ID: 25366 10.D

Date Collected: 06/02/2017 10:50

Date Analyzed: 06/06/2017 18:51

Soil Extract Vol.:

Dilution Factor: 1

\% Moisture:

GC Column: DB-624

ID: $0.2(\mathrm{~mm})$

Analysis Batch No.: 117296

Level: (low/med) Trace

Units: ug/L

\begin{tabular}{|c|c|c|c|c|c|}
\hline CAS NO. & COMPOUND NAME & $\mathrm{RT}$ & RESULT & Q & $\begin{array}{c}\text { MATCH } \\
\text { QUALITY }\end{array}$ \\
\hline STL00989 & Total Alkanes & & & & \\
\hline
\end{tabular}


FORM I

GC/MS VOA ORGANICS ANALYSIS DATA SHEET

Lab Name: TestAmerica Burlington

SDG No.: 200-38792-1

Client Sample ID: MRQCTB-W-39813

Matrix: Water

Analysis Method: SOM02.2/VOA_Tr

Sample wt/vol: 25 (mL)

Soil Aliquot Vol:

Soil Extract Vol.:

응 Moisture:

Analysis Batch No.: 117296
Job No.: 200-38792-1

Lab Sample ID: 200-38792-4

Lab File ID: 25366_11.D

Date Collected: 06/02/2017 10:00

Date Analyzed: 06/06/2017 19:17

Dilution Factor: 1

GC Column: DB-624

ID: $0.2(\mathrm{~mm})$

Level: (low/med) Trace

Units: ug/L

\begin{tabular}{|c|c|c|c|c|}
\hline CAS NO. & COMPOUND NAME & RESULT & $Q$ & $\mathrm{RL}$ \\
\hline $75-71-8$ & Dichlorodifluoromethane & 0.50 & $\mathrm{U}$ & 0.50 \\
\hline $74-87-3$ & Chloromethane & 0.50 & $\mathrm{U}$ & 0.50 \\
\hline $75-01-4$ & Vinyl chloride & 0.50 & $\mathrm{U}$ & 0.50 \\
\hline $74-83-9$ & Bromomethane & 0.50 & $\mathrm{U}$ & 0.50 \\
\hline $75-00-3$ & Chloroethane & 0.50 & $\mathrm{U}$ & 0.50 \\
\hline $75-69-4$ & Trichlorofluoromethane & 0.50 & $\mathrm{U}$ & 0.50 \\
\hline $75-35-4$ & 1,1-Dichloroethene & 0.50 & $\mathrm{U}$ & 0.50 \\
\hline $76-13-1$ & $\begin{array}{l}\text { 1, 1,2-Trichloro-1,2,2-trifluoroethan } \\
\text { e }\end{array}$ & 0.50 & $\mathrm{U}$ & 0.50 \\
\hline $67-64-1$ & Acetone & 5.1 & B & 5.0 \\
\hline $75-15-0$ & Carbon disulfide & 0.10 & $\mathrm{~J} B$ & 0.50 \\
\hline $79-20-9$ & Methyl acetate & 0.50 & $\mathrm{U}$ & 0.50 \\
\hline $75-09-2$ & Methylene Chloride & 0.50 & $\mathrm{U}$ & 0.50 \\
\hline $156-60-5$ & trans-1,2-Dichloroethene & 0.50 & $\mathrm{U}$ & 0.50 \\
\hline $1634-04-4$ & Methyl tert-butyl ether & 0.50 & $\mathrm{U}$ & 0.50 \\
\hline $75-34-3$ & 1,1-Dichloroethane & 0.50 & $\mathrm{U}$ & 0.50 \\
\hline $156-59-2$ & cis-1,2-Dichloroethene & 0.50 & $\mathrm{U}$ & 0.50 \\
\hline $78-93-3$ & 2-Butanone & 5.0 & $\mathrm{U}$ & 5.0 \\
\hline $74-97-5$ & Bromochloromethane & 0.50 & $\mathrm{U}$ & 0.50 \\
\hline $67-66-3$ & Chloroform & 0.21 & $\mathrm{~J}$ & 0.50 \\
\hline $71-55-6$ & $1,1,1$-Trichloroethane & 0.50 & $\mathrm{U}$ & 0.50 \\
\hline $110-82-7$ & Cyclohexane & 0.50 & $\mathrm{U}$ & 0.50 \\
\hline $56-23-5$ & Carbon tetrachloride & 0.50 & $\mathrm{U}$ & 0.50 \\
\hline $71-43-2$ & Benzene & 0.23 & $\mathrm{~J}$ & 0.50 \\
\hline $107-06-2$ & 1,2-Dichloroethane & 0.50 & $\mathrm{U}$ & 0.50 \\
\hline $79-01-6$ & Trichloroethene & 0.50 & $\mathrm{U}$ & 0.50 \\
\hline $108-87-2$ & Methylcyclohexane & 0.50 & $\mathrm{U}$ & 0.50 \\
\hline $78-87-5$ & 1,2-Dichloropropane & 0.50 & $\mathrm{U}$ & 0.50 \\
\hline $75-27-4$ & Bromodichloromethane & 0.50 & $\mathrm{U}$ & 0.50 \\
\hline $10061-01-5$ & cis-1,3-Dichloropropene & 0.50 & $\mathrm{U}$ & 0.50 \\
\hline $108-10-1$ & 4-Methyl-2-pentanone & 5.0 & $\mathrm{U}$ & 5.0 \\
\hline $108-88-3$ & Toluene & 1.2 & & 0.50 \\
\hline $10061-02-6$ & trans-1,3-Dichloropropene & 0.50 & $\mathrm{U}$ & 0.50 \\
\hline $79-00-5$ & 1,1,2-Trichloroethane & 0.50 & $\mathrm{U}$ & 0.50 \\
\hline $127-18-4$ & Tetrachloroethene & 0.50 & $\mathrm{U}$ & 0.50 \\
\hline $591-78-6$ & 2-Hexanone & 5.0 & $\mathrm{U}$ & 5.0 \\
\hline
\end{tabular}


FORM I

GC/MS VOA ORGANICS ANALYSIS DATA SHEET

Lab Name: TestAmerica Burlington

SDG No.: 200-38792-1

Client Sample ID: MRQCTB-W-39813

Matrix: Water

Analysis Method: SOM02.2/VOA_Tr

Sample wt/vol: 25 (mL)

Soil Aliquot Vol:

Soil Extract Vol.:

응 Moisture:

Analysis Batch No.: 117296
Job No.: 200-38792-1

Lab Sample ID: 200-38792-4

Lab File ID: 25366 11.D

Date Collected: 06/02/2017 10:00

Date Analyzed: 06/06/2017 19:17

Dilution Factor: 1

GC Column: DB-624

ID: $0.2(\mathrm{~mm})$

Level: (low/med) Trace

Units: ug/L

\begin{tabular}{|c|c|c|c|c|}
\hline CAS NO. & COMPOUND NAME & RESULT & $Q$ & RL \\
\hline $124-48-1$ & Dibromochloromethane & 0.50 & $\mathrm{U}$ & 0.50 \\
\hline $106-93-4$ & 1,2-Dibromoethane & 0.50 & $\mathrm{U}$ & 0.50 \\
\hline $108-90-7$ & Chlorobenzene & 0.50 & $\mathrm{U}$ & 0.50 \\
\hline $100-41-4$ & Ethylbenzene & 0.079 & $\mathrm{~J}$ & 0.50 \\
\hline $95-47-6$ & o-Xylene & 0.12 & $\mathrm{~J}$ & 0.50 \\
\hline $179601-23-1$ & m,p-Xylene & 0.31 & $\mathrm{~J}$ & 0.50 \\
\hline $100-42-5$ & Styrene & 0.50 & $\mathrm{U}$ & 0.50 \\
\hline $75-25-2$ & Bromoform & 0.50 & $\mathrm{U}$ & 0.50 \\
\hline $98-82-8$ & Isopropylbenzene & 0.50 & $\mathrm{U}$ & 0.50 \\
\hline $79-34-5$ & $1,1,2,2$-Tetrachloroethane & 0.50 & $\mathrm{U}$ & 0.50 \\
\hline $541-73-1$ & 1,3-Dichlorobenzene & 0.50 & $\mathrm{U}$ & 0.50 \\
\hline $106-46-7$ & 1,4-Dichlorobenzene & 0.50 & $\mathrm{U}$ & 0.50 \\
\hline $95-50-1$ & 1,2-Dichlorobenzene & 0.50 & $\mathrm{U}$ & 0.50 \\
\hline $96-12-8$ & 1,2-Dibromo-3-chloropropane & 0.50 & $\mathrm{U}$ & 0.50 \\
\hline $120-82-1$ & $1,2,4$-Trichlorobenzene & 0.50 & $\mathrm{U}$ & 0.50 \\
\hline $87-61-6$ & $1,2,3$-Trichlorobenzene & 0.50 & $\mathrm{U}$ & 0.50 \\
\hline
\end{tabular}

\begin{tabular}{|c|c|c|c|c|}
\hline CAS NO. & SURROGATE & $\because \mathrm{REC}$ & Q & LIMITS \\
\hline $6745-35-3$ & Vinyl chloride-d3 & 108 & & $40-130$ \\
\hline $19199-91-8$ & Chloroethane-d5 & 125 & & $65-130$ \\
\hline $22280-73-5$ & 1,1-Dichloroethene-d2 & 96 & & $60-125$ \\
\hline $24313-50-6$ & 2-Butanone-d5 & 155 & * & $40-130$ \\
\hline $865-49-6$ & Chloroform-d & 127 & * & $70-125$ \\
\hline $17060-07-0$ & 1,2-Dichloroethane-d4 & 142 & * & $70-130$ \\
\hline $1076-43-3$ & Benzene-d6 & 120 & & $70-125$ \\
\hline $93952-08-0$ & 1,2-Dichloropropane-d6 & 97 & & $60-140$ \\
\hline $2037-26-5$ & Toluene-d8 & 119 & & $70-130$ \\
\hline $93951-86-1$ & trans-1,3-Dichloropropene-d4 & 115 & & $55-130$ \\
\hline $4840-82-8$ & 2-Hexanone-d5 & 158 & * & $45-130$ \\
\hline $33685-54-0$ & $1,1,2,2$-Tetrachloroethane-d2 & 115 & & $65-120$ \\
\hline $2199-69-1$ & 1,2-Dichlorobenzene-d4 & 119 & & $80-120$ \\
\hline
\end{tabular}


FORM I

GC/MS VOA ORGANICS ANALYSIS DATA SHEET

TENTATIVELY IDENTIFIED COMPOUNDS

Lab Name: TestAmerica Burlington

SDG No.: 200-38792-1

Client Sample ID: MRQCTB-W-39813

Matrix: Water

Analysis Method: SOM02.2/VOA_Tr

Sample wt/vol: $25(\mathrm{~mL})$

Soil Aliquot Vol:

Soil Extract Vol.:

\% Moisture:

Analysis Batch No.: 117296

Number TICS Found: 2
Job No.: 200-38792-1

Lab Sample ID: 200-38792-4

Lab File ID: 25366_11.D

Date Collected: 06/02/2017 10:00

Date Analyzed: 06/06/2017 19:17

Dilution Factor: 1

GC Column: DB-624

ID: $0.2(\mathrm{~mm})$

Level: (low/med) Trace

Units: ug/L

TIC Result Total: 5.1

\begin{tabular}{|c|c|c|c|c|c|}
\hline CAS NO. & COMPOUND NAME & $\mathrm{RT}$ & RESULT & Q & $\begin{array}{c}\text { MATCH } \\
\text { QUALITY }\end{array}$ \\
\hline $109-67-1$ & 1-Pentene & 3.30 & 2.0 & $\mathrm{~J} \mathrm{~N}$ & $74 \%$ \\
\hline & Unknown & 7.28 & 3.1 & $\mathrm{JX}$ & \\
\hline
\end{tabular}


FORM I

GC/MS VOA ORGANICS ANALYSIS DATA SHEET

TARGETED TENATIVELY IDENTIFIED COMPOUNDS

Lab Name: TestAmerica Burlington

SDG No.: 200-38792-1

Client Sample ID: MRQCTB-W-39813

Matrix: Water

Analysis Method: SOM02.2/VOA_Tr

Sample wt/vol: 25 (mL)

Soil Aliquot Vol:

Soil Extract Vol.:

․ Moisture:

Analysis Batch No.: 117296
Job No.: 200-38792-1

Lab Sample ID: 200-38792-4

Lab File ID: 25366 11.D

Date Collected: 06/02/2017 10:00

Date Analyzed: 06/06/2017 19:17

Dilution Factor: 1

GC Column: DB-624

ID: $0.2(\mathrm{~mm})$

Level: (low/med) Trace

Units: ug/L

\begin{tabular}{|c|c|c|c|c|}
\hline CAS NO. & COMPOUND NAME & RT & RESULT & Q \\
\hline QUALITY
\end{tabular}


FORM I

GC/MS VOA ORGANICS ANALYSIS DATA SHEET

Lab Name: TestAmerica Burlington

SDG No.: 200-38792-1

Client Sample ID: MRQCTB-W-39813

Matrix: Water

Analysis Method: SOM02.2/VOA_Tr

Sample wt/vol: 2 (uL)

Soil Aliquot Vol:

Soil Extract Vol.:

응 Moisture:

Analysis Batch No.: 117409
Job No.: 200-38792-1

Lab Sample ID: 200-38792-4

Lab File ID: 25405_21.D

Date Collected: 06/02/2017 10:00

Date Analyzed: 06/08/2017 22:50

Dilution Factor: 1

GC Column: DB-624

ID: $0.2(\mathrm{~mm})$

Level: (low/med) Trace

Units: ug/L

\begin{tabular}{|c|c|c|c|c|c|}
\hline CAS NO. & COMPOUND NAME & RESULT & $Q$ & RL & \\
\hline $75-71-8$ & Dichlorodifluoromethane & 0.50 & $U$ & 0.50 & \\
\hline $74-87-3$ & Chloromethane & 0.50 & $U$ & 0.50 & \\
\hline $75-01-4$ & Vinyl chloride & 0.50 & $U$ & 0.50 & \\
\hline $74-83-9$ & Bromomethane & 0.50 & $U$ & 0.50 & \\
\hline $75-00-3$ & Chloroethane & 0.50 & $U$ & 0.50 & \\
\hline $75-69-4$ & Trichlorofluoromethane & 0.50 & $U$ & 0.50 & \\
\hline $75-35-4$ & 1,1-Dichloroethene & 0.50 & $U$ & 0.50 & \\
\hline $76-13-1$ & $\begin{array}{l}\text { 1,1,2-Trichloro-1,2,2-trifluoroethan } \\
e\end{array}$ & 0.50 & $U$ & 0.50 & \\
\hline $67-64-1$ & Acetone & 4.9 & $J B$ & 5.0 & \\
\hline $75-15-0$ & Carbon disulfide & 0.15 & $J B$ & 0.50 & \\
\hline $79-20-9$ & Methyl acetate & 0.50 & $U$ & 0.50 & \\
\hline $75-09-2$ & Methylene Chloride & 0.50 & $U$ & 0.50 & \\
\hline $156-60-5$ & trans-1,2-Dichloroethene & 0.50 & $U$ & 0.50 & \\
\hline $1634-04-4$ & Methyl tert-butyl ether & 0.50 & $U$ & 0.50 & \\
\hline $75-34-3$ & 1,1-Dichloroethane & 0.50 & $U$ & 0.50 & \\
\hline $156-59-2$ & cis-1,2-Dichloroethene & 0.50 & $U$ & 0.50 & \\
\hline $78-93-3$ & 2-Butanone & 5.0 & $U$ & 5.0 & \\
\hline $74-97-5$ & Bromochloromethane & 0.50 & $U$ & 0.50 & \\
\hline $67-66-3$ & Chloroform & 0.50 & $U$ & 0.50 & \\
\hline $71-55-6$ & 1,1,1-Trichloroethane & 0.50 & $U$ & 0.50 & \\
\hline $110-82-7$ & Cyclohexane & 0.50 & $U$ & 0.50 & \\
\hline $56-23-5$ & Carbon tetrachloride & 0.50 & $U$ & 0.50 & \\
\hline $71-43-2$ & Benzene & 0.23 & $\mathrm{~J}$ & 0.50 & \\
\hline $107-06-2$ & 1,2-Dichloroethane & 0.50 & $U$ & 0.50 & \\
\hline $79-01-6$ & Trichloroethene & 0.50 & $U$ & 0.50 & \\
\hline $108-87-2$ & Methylcyclohexane & 0.50 & $U$ & 0.50 & \\
\hline $78-87-5$ & 1,2-Dichloropropane & 0.50 & $U$ & 0.50 & \\
\hline $75-27-4$ & Bromodichloromethane & 0.50 & $U$ & 0.50 & \\
\hline $10061-01-5$ & cis-1,3-Dichloropropene & 0.50 & $U$ & 0.50 & \\
\hline $108-10-1$ & 4-Methyl-2-pentanone & 5.0 & $U$ & 5.0 & \\
\hline $108-88-3$ & Toluene & 1.2 & & 0.50 & \\
\hline $10061-02-6$ & trans-1,3-Dichloropropene & 0.50 & $U$ & 0.50 & \\
\hline $79-00-5$ & 1,1,2-Trichloroethane & 0.50 & $U$ & 0.50 & \\
\hline $127-18-4$ & Tetrachloroethene & 0.50 & $U$ & 0.50 & \\
\hline $591-78-6$ & 2-Hexanone & 5.0 & $U$ & 5.0 & \\
\hline
\end{tabular}

FORM I SOM02.2/VOA_Tr 
FORM I

GC/MS VOA ORGANICS ANALYSIS DATA SHEET

Lab Name: TestAmerica Burlington

SDG No.: 200-38792-1

Client Sample ID: MRQCTB-W-39813

Matrix: Water

Analysis Method: SOM02.2/VOA_Tr

Sample wt/vol: 2 (uL)

Soil Aliquot Vol:

Soil Extract Vol.:

응 Moisture:

Analysis Batch No.: 117409
Job No. : 200-38792-1

Lab Sample ID: 200-38792-4

Lab File ID: 25405_21.D

Date Collected: 06/02/2017 10:00

Date Analyzed: 06/08/2017 22:50

Dilution Factor: 1

GC Column: DB-624

ID: $0.2(\mathrm{~mm})$

Level: (low/med) Trace

Units: ug/L

\begin{tabular}{|c|c|c|c|c|}
\hline CAS NO. & COMPOUND NAME & RESULT & $Q$ & RL \\
\hline $124-48-1$ & Dibromochloromethane & 0.50 & $U$ & 0.50 \\
\hline $106-93-4$ & 1,2-Dibromoethane & 0.50 & $U$ & 0.50 \\
\hline $108-90-7$ & Chlorobenzene & 0.50 & $U$ & 0.50 \\
\hline $100-41-4$ & Ethylbenzene & 0.087 & $\mathrm{~J}$ & 0.50 \\
\hline $95-47-6$ & o-Xylene & 0.12 & J & 0.50 \\
\hline $179601-23-1$ & $m, p-X y l e n e$ & 0.35 & J & 0.50 \\
\hline $100-42-5$ & Styrene & 0.50 & $U$ & 0.50 \\
\hline $75-25-2$ & Bromoform & 0.50 & $U$ & 0.50 \\
\hline $98-82-8$ & Isopropylbenzene & 0.50 & $U$ & 0.50 \\
\hline $79-34-5$ & $1,1,2,2$-Tetrachloroethane & 0.50 & $U$ & 0.50 \\
\hline $541-73-1$ & 1,3-Dichlorobenzene & 0.50 & $U$ & 0.50 \\
\hline $106-46-7$ & 1,4-Dichlorobenzene & 0.50 & $U$ & 0.50 \\
\hline $95-50-1$ & 1,2-Dichlorobenzene & 0.50 & $U$ & 0.50 \\
\hline $96-12-8$ & 1,2-Dibromo-3-chloropropane & 0.50 & $U$ & 0.50 \\
\hline $120-82-1$ & $1,2,4-$ Trichlorobenzene & 0.50 & $U$ & 0.50 \\
\hline $87-61-6$ & $1,2,3-$ Trichlorobenzene & 0.50 & U & 0.50 \\
\hline
\end{tabular}

\begin{tabular}{|c|c|c|c|c|}
\hline CAS NO. & SURROGATE & $\because \mathrm{REC}$ & Q & LIMITS \\
\hline $6745-35-3$ & Vinyl chloride-d3 & 78 & & $40-130$ \\
\hline $19199-91-8$ & Chloroethane-d5 & 109 & & $65-130$ \\
\hline $22280-73-5$ & 1,1-Dichloroethene-d2 & 89 & & $60-125$ \\
\hline $24313-50-6$ & 2-Butanone-d5 & 124 & & $40-130$ \\
\hline $865-49-6$ & Chloroform-d & 117 & & $70-125$ \\
\hline $17060-07-0$ & $1,2-$ Dichloroethane-d4 & 137 & * & $70-130$ \\
\hline $1076-43-3$ & Benzene-d6 & 112 & & $70-125$ \\
\hline $93952-08-0$ & 1,2-Dichloropropane-d6 & 89 & & $60-140$ \\
\hline $2037-26-5$ & Toluene-d8 & 112 & & $70-130$ \\
\hline $93951-86-1$ & trans-1,3-Dichloropropene-d4 & 110 & & $55-130$ \\
\hline $4840-82-8$ & 2-Hexanone-d5 & 132 & * & $45-130$ \\
\hline $33685-54-0$ & $1,1,2,2$-Tetrachloroethane-d2 & 109 & & $65-120$ \\
\hline $2199-69-1$ & 1,2-Dichlorobenzene-d4 & 117 & & $80-120$ \\
\hline
\end{tabular}


FORM I

GC/MS VOA ORGANICS ANALYSIS DATA SHEET

TENTATIVELY IDENTIFIED COMPOUNDS

Lab Name: TestAmerica Burlington

SDG No.: 200-38792-1

Client Sample ID: MRQCTB-W-39813

Matrix: Water

Analysis Method: SOM02.2/VOA_Tr

Sample wt/vol: 2 (uL)

Soil Aliquot Vol:

Soil Extract Vol.:

\% Moisture:

Analysis Batch No.: 117409

Number TICs Found: 2
Job No.: 200-38792-1

Lab Sample ID: 200-38792-4

Lab File ID: 25405 21.D

Date Collected: 06/02/2017 10:00

Date Analyzed: 06/08/2017 22:50

Dilution Factor: 1

GC Column: DB-624

ID $: 0.2(\mathrm{~mm})$

Level: (low/med) Trace

Units: ug/L

TIC Result Total: 3.41

\begin{tabular}{|c|c|c|c|c|c|}
\hline CAS NO. & COMPOUND NAME & $\mathrm{RT}$ & RESULT & $Q$ & $\begin{array}{c}\text { MATCH } \\
\text { QUALITY }\end{array}$ \\
\hline & Unknown & 1.64 & 0.51 & $\mathrm{~J}$ & \\
\hline & Unknown & 7.28 & 2.9 & $\mathrm{~J} X$ & \\
\hline
\end{tabular}


FORM I

GC/MS VOA ORGANICS ANALYSIS DATA SHEET

TARGETED TENATIVELY IDENTIFIED COMPOUNDS

Lab Name: TestAmerica Burlington

SDG No.: 200-38792-1

Client Sample ID: MRQCTB-W-39813

Matrix: Water

Analysis Method: SOM02.2/VOA_Tr

Sample wt/vol: 2 (uL)

Soil Aliquot Vol:

Soil Extract Vol.:

응 Moisture:

Analysis Batch No.: 117409
Job No.: 200-38792-1

Lab Sample ID: 200-38792-4

Lab File ID: 25405 21.D

Date Collected: 06/02/2017 10:00

Date Analyzed: 06/08/2017 22:50

Dilution Factor: 1

GC Column: DB-624

ID: $0.2(\mathrm{~mm})$

Level: (low/med) Trace

Units: ug/L

\begin{tabular}{|c|c|c|c|c|}
\hline CAS NO. & COMPOUND NAME & RT & RESULT & Q \\
\hline QUALITY
\end{tabular}


FORM I

GC/MS VOA ORGANICS ANALYSIS DATA SHEET

Lab Name: TestAmerica Burlington

Job No : : 200-38792-1

SDG No.: 200-38792-1

client sample ID: VHBLK01

Matrix: Water

Analysis Method: SOM02.2/VOA_Tr

Sample wt/vol: 25 (mL)

Soil Aliquot Vol:

Soil Extract Vol.:

․ Moisture:

Analysis Batch No.: 117296

Lab Sample ID: 200-38792-5

Lab File ID: 25366_12.D

Date Collected: 06/03/2017 11:08

Date Analyzed: 06/06/2017 19:43

Dilution Factor: 1

GC Column: DB-624

ID: $0.2(\mathrm{~mm})$

Level: (low/med) Trace

Units: ug/L

\begin{tabular}{|c|c|c|c|c|}
\hline CAS NO. & COMPOUND NAME & RESULT & $Q$ & RL \\
\hline $75-71-8$ & Dichlorodifluoromethane & 0.50 & $\mathrm{U}$ & 0.50 \\
\hline $74-87-3$ & Chloromethane & 0.50 & $\mathrm{U}$ & 0.50 \\
\hline $75-01-4$ & Vinyl chloride & 0.50 & $\mathrm{U}$ & 0.50 \\
\hline $74-83-9$ & Bromomethane & 0.50 & $\mathrm{U}$ & 0.50 \\
\hline $75-00-3$ & Chloroethane & 0.50 & $\mathrm{U}$ & 0.50 \\
\hline $75-69-4$ & Trichlorofluoromethane & 0.50 & $\mathrm{U}$ & 0.50 \\
\hline $75-35-4$ & 1,1-Dichloroethene & 0.50 & $\mathrm{U}$ & 0.50 \\
\hline $76-13-1$ & $\begin{array}{l}\text { 1, 1,2-Trichloro-1,2,2-trifluoroethan } \\
\text { e }\end{array}$ & 0.50 & $\mathrm{U}$ & 0.50 \\
\hline $67-64-1$ & Acetone & 2.9 & $\mathrm{~J} B$ & 5.0 \\
\hline $75-15-0$ & Carbon disulfide & 0.10 & $\mathrm{~J} B$ & 0.50 \\
\hline $79-20-9$ & Methyl acetate & 0.50 & $\mathrm{U}$ & 0.50 \\
\hline $75-09-2$ & Methylene Chloride & 0.50 & $\mathrm{U}$ & 0.50 \\
\hline $156-60-5$ & trans-1,2-Dichloroethene & 0.50 & $\mathrm{U}$ & 0.50 \\
\hline $1634-04-4$ & Methyl tert-butyl ether & 0.50 & $\mathrm{U}$ & 0.50 \\
\hline $75-34-3$ & 1,1-Dichloroethane & 0.50 & $\mathrm{U}$ & 0.50 \\
\hline $156-59-2$ & cis-1,2-Dichloroethene & 0.50 & $\mathrm{U}$ & 0.50 \\
\hline $78-93-3$ & 2-Butanone & 5.0 & $\mathrm{U}$ & 5.0 \\
\hline $74-97-5$ & Bromochloromethane & 0.50 & $\mathrm{U}$ & 0.50 \\
\hline $67-66-3$ & Chloroform & 0.50 & $\mathrm{U}$ & 0.50 \\
\hline $71-55-6$ & $1,1,1$-Trichloroethane & 0.50 & $\mathrm{U}$ & 0.50 \\
\hline $110-82-7$ & Cyclohexane & 0.50 & $\mathrm{U}$ & 0.50 \\
\hline $56-23-5$ & Carbon tetrachloride & 0.50 & $\mathrm{U}$ & 0.50 \\
\hline $71-43-2$ & Benzene & 0.50 & $\mathrm{U}$ & 0.50 \\
\hline $107-06-2$ & 1,2-Dichloroethane & 0.50 & $\mathrm{U}$ & 0.50 \\
\hline $79-01-6$ & Trichloroethene & 0.50 & $\mathrm{U}$ & 0.50 \\
\hline $108-87-2$ & Methylcyclohexane & 0.50 & $\mathrm{U}$ & 0.50 \\
\hline $78-87-5$ & 1,2-Dichloropropane & 0.50 & $\mathrm{U}$ & 0.50 \\
\hline $75-27-4$ & Bromodichloromethane & 0.50 & $\mathrm{U}$ & 0.50 \\
\hline $10061-01-5$ & cis-1,3-Dichloropropene & 0.50 & $\mathrm{U}$ & 0.50 \\
\hline $108-10-1$ & 4-Methyl-2-pentanone & 5.0 & $\mathrm{U}$ & 5.0 \\
\hline $108-88-3$ & Toluene & 0.50 & $\mathrm{U}$ & 0.50 \\
\hline $10061-02-6$ & trans-1,3-Dichloropropene & 0.50 & $\mathrm{U}$ & 0.50 \\
\hline $79-00-5$ & 1,1,2-Trichloroethane & 0.50 & $\mathrm{U}$ & 0.50 \\
\hline $127-18-4$ & Tetrachloroethene & 0.50 & $\mathrm{U}$ & 0.50 \\
\hline $591-78-6$ & 2-Hexanone & 5.0 & $\mathrm{U}$ & 5.0 \\
\hline
\end{tabular}

FORM I SOM02.2/VOA_Tr 
FORM I

GC/MS VOA ORGANICS ANALYSIS DATA SHEET

Lab Name: TestAmerica Burlington

SDG No.: 200-38792-1

Client Sample ID: VHBLKO1

Matrix: Water

Analysis Method: SOM02.2/VOA_Tr

Sample wt/vol: 25 (mL)

Soil Aliquot Vol:

Soil Extract Vol.:

응 Moisture:

Analysis Batch No.: 117296
Job No.: 200-38792-1

Lab Sample ID: 200-38792-5

Lab File ID: 25366_12.D

Date Collected: 06/03/2017 11:08

Date Analyzed: 06/06/2017 19:43

Dilution Factor: 1

GC Column: DB-624

ID: $0.2(\mathrm{~mm})$

Level: (low/med) Trace

Units: ug/L

\begin{tabular}{|c|c|c|c|c|}
\hline CAS NO. & COMPOUND NAME & RESULT & $Q$ & RL \\
\hline $124-48-1$ & Dibromochloromethane & 0.50 & $\mathrm{U}$ & 0.50 \\
\hline $106-93-4$ & 1,2-Dibromoethane & 0.50 & $\mathrm{U}$ & 0.50 \\
\hline $108-90-7$ & Chlorobenzene & 0.50 & $\mathrm{U}$ & 0.50 \\
\hline $100-41-4$ & Ethylbenzene & 0.50 & $\mathrm{U}$ & 0.50 \\
\hline $95-47-6$ & o-Xylene & 0.50 & $\mathrm{U}$ & 0.50 \\
\hline $179601-23-1$ & m,p-Xylene & 0.50 & $\mathrm{U}$ & 0.50 \\
\hline $100-42-5$ & Styrene & 0.50 & $\mathrm{U}$ & 0.50 \\
\hline $75-25-2$ & Bromoform & 0.50 & $\mathrm{U}$ & 0.50 \\
\hline $98-82-8$ & Isopropylbenzene & 0.50 & $\mathrm{U}$ & 0.50 \\
\hline $79-34-5$ & $1,1,2,2$-Tetrachloroethane & 0.50 & $\mathrm{U}$ & 0.50 \\
\hline $541-73-1$ & 1,3-Dichlorobenzene & 0.50 & $\mathrm{U}$ & 0.50 \\
\hline $106-46-7$ & 1,4-Dichlorobenzene & 0.50 & $\mathrm{U}$ & 0.50 \\
\hline $95-50-1$ & 1,2-Dichlorobenzene & 0.50 & $\mathrm{U}$ & 0.50 \\
\hline $96-12-8$ & 1,2-Dibromo-3-chloropropane & 0.50 & $\mathrm{U}$ & 0.50 \\
\hline $120-82-1$ & $1,2,4$-Trichlorobenzene & 0.50 & $\mathrm{U}$ & 0.50 \\
\hline $87-61-6$ & $1,2,3$-Trichlorobenzene & 0.50 & $\mathrm{U}$ & 0.50 \\
\hline
\end{tabular}

\begin{tabular}{|c|c|c|c|c|}
\hline CAS NO. & SURROGATE & $\because \mathrm{REC}$ & Q & LIMITS \\
\hline $6745-35-3$ & Vinyl chloride-d3 & 102 & & $40-130$ \\
\hline $19199-91-8$ & Chloroethane-d5 & 118 & & $65-130$ \\
\hline $22280-73-5$ & 1,1-Dichloroethene-d2 & 93 & & $60-125$ \\
\hline $24313-50-6$ & 2-Butanone-d5 & 123 & & $40-130$ \\
\hline $865-49-6$ & Chloroform-d & 122 & & $70-125$ \\
\hline $17060-07-0$ & 1,2-Dichloroethane-d4 & 137 & * & $70-130$ \\
\hline $1076-43-3$ & Benzene-d6 & 119 & & $70-125$ \\
\hline $93952-08-0$ & 1,2-Dichloropropane-d6 & 96 & & $60-140$ \\
\hline $2037-26-5$ & Toluene-d8 & 116 & & $70-130$ \\
\hline $93951-86-1$ & trans-1,3-Dichloropropene-d4 & 108 & & $55-130$ \\
\hline $4840-82-8$ & 2-Hexanone-d5 & 125 & & $45-130$ \\
\hline $33685-54-0$ & $1,1,2,2$-Tetrachloroethane-d2 & 110 & & $65-120$ \\
\hline $2199-69-1$ & 1,2 -Dichlorobenzene-d4 & 121 & * & $80-120$ \\
\hline
\end{tabular}


FORM I

GC/MS VOA ORGANICS ANALYSIS DATA SHEET

TENTATIVELY IDENTIFIED COMPOUNDS

Lab Name: TestAmerica Burlington

SDG No.: 200-38792-1

Client Sample ID: VHBLKO1

Matrix: Water

Analysis Method: SOM02.2/VOA_Tr

Sample wt/vol: $25(\mathrm{~mL})$

Soil Aliquot Vol:

Soil Extract Vol.:

\% Moisture:

Analysis Batch No.: 117296

Number TICs Found: 1

\begin{tabular}{|c|c|c|c|c|}
\hline CAS NO. & COMPOUND NAME & RT & RESULT & Q \\
QUATCHTY \\
\hline \hline
\end{tabular}

Job No.: 200-38792-1

Lab Sample ID: 200-38792-5

Lab File ID: 25366_12.D

Date Collected: 06/03/2017 11:08

Date Analyzed: 06/06/2017 19:43

Dilution Factor: 1

GC Column: DB-624

ID $: 0.2(\mathrm{~mm})$

Level: (low/med) Trace

Units: ug/L

TIC Result Total: 2.9 
FORM I

GC/MS VOA ORGANICS ANALYSIS DATA SHEET

TARGETED TENATIVELY IDENTIFIED COMPOUNDS

Lab Name: TestAmerica Burlington

SDG No.: 200-38792-1

Client Sample ID: VHBLK01

Matrix: Water

Analysis Method: SOM02.2/VOA_Tr

Sample wt/vol: 25 (mL)

Soil Aliquot Vol:

Soil Extract Vol.:

\% Moisture:

Analysis Batch No.: 117296
Job No.: 200-38792-1

Lab Sample ID: 200-38792-5

Lab File ID: 25366 12.D

Date Collected: 06/03/2017 11:08

Date Analyzed: 06/06/2017 19:43

Dilution Factor: 1

GC Column: DB-624

ID $: 0.2(\mathrm{~mm})$

Level: (low/med) Trace

Units: ug/L

\begin{tabular}{|c|c|c|c|c|}
\hline CAS NO. & COMPOUND NAME & RT & RESULT & Q \\
\hline QUALITY
\end{tabular}


FORM VI

GC/MS VOA BY INTERNAL STANDARD - INITIAL CALIBRATION DATA

CURVE EVALUATION

Lab Name: TestAmerica Burlington

Job No.: 200-38792-1

Analy Batch No.: 116931

SDG No.: 200-38792-1

Instrument ID: CHD.i

GC Column: DB-624

ID $: 0.2(\mathrm{~mm})$

Heated Purge: (Y/N) N

Calibration Start Date: 05/23/2017 17:32

Calibration End Date: 05/23/2017 19:16

Calibration ID: 37252

Calibration Files:

\begin{tabular}{|llll|}
\hline LEVEL: & LAB SAMPLE ID: & EPA SAMPLE NO: & LAB FILE ID: \\
Level 1 & IC 200-116931/30 & VSTDO.5EA & 25203_11.D \\
Level 2 & IC 200-116931/29 & VSTD001EA & 25203_10.D \\
Level 3 & ICIS 200-116931/28 & VSTD005EA & 25203_09.D \\
Level 4 & IC 200-116931/27 & VSTD010EA & 25203_08.D \\
Level 5 & IC 200-116931/26 & VSTD020EA & 25203_07.D \\
\hline
\end{tabular}

\begin{tabular}{|c|c|c|c|c|c|c|c|c|c|c|c|c|c|c|c|c|c|}
\hline \multirow[t]{2}{*}{ ANALYTE } & \multicolumn{5}{|c|}{$\mathrm{RRF}$} & \multirow{2}{*}{\begin{tabular}{|l|} 
CURVE \\
TYPE
\end{tabular}} & \multicolumn{3}{|c|}{ COEFFICIENT } & \multirow[t]{2}{*}{$\#$} & \multirow[t]{2}{*}{ MIN RRF } & \multirow[t]{2}{*}{$\div$ RSD } & \multirow[t]{2}{*}{$\#$} & \multirow{2}{*}{\begin{tabular}{|c|} 
MAX \\
$\circ R S D$
\end{tabular}} & \multirow{2}{*}{\begin{tabular}{|c|}
$R^{\wedge} 2$ \\
$O R \quad C O D$
\end{tabular}} & \multirow[t]{2}{*}{$\#$} & \multirow{2}{*}{$\begin{array}{ccc}\text { MIN } & R^{\wedge} 2 \\
\text { OR } & \text { COD }\end{array}$} \\
\hline & LVL 1 & LVL 2 & LVL 3 & LVL 4 & LVL 5 & & $\mathrm{~B}$ & M1 & M2 & & & & & & & & \\
\hline Dichlorodifluoromethane & 0.2956 & 0.3196 & 0.3169 & 0.3352 & 0.3021 & Ave & & 0.3139 & & & 0.0100 & 5.0 & & 30.0 & & & \\
\hline Chloromethane & 0.2639 & 0.2607 & 0.2244 & 0.2431 & 0.2157 & Ave & & 0.2416 & & & 0.0100 & 8.8 & & 30.0 & & & \\
\hline Vinyl chloride & 0.2201 & 0.2321 & 0.2264 & 0.2450 & 0.2209 & Ave & & 0.2289 & & & 0.0100 & 4.5 & & 30.0 & & & \\
\hline Bromomethane & 0.1007 & 0.0968 & 0.0936 & 0.1022 & 0.0892 & Ave & & 0.0965 & & & 0.0100 & 5.5 & & 40.0 & & & \\
\hline Chloroethane & 0.1458 & 0.1368 & 0.1305 & 0.1368 & 0.1197 & Ave & & 0.1339 & & & 0.0100 & 7.2 & & 30.0 & & & \\
\hline Trichlorofluoromethane & 0.4857 & 0.5069 & 0.4886 & 0.5098 & 0.4673 & Ave & & 0.4917 & & & 0.0100 & 3.5 & & 30.0 & & & \\
\hline 1,1,2-Trichloro-1,2,2-trifluoroethane & 0.2264 & 0.2711 & 0.2519 & 0.2567 & 0.2307 & Ave & & 0.2474 & & & 0.0100 & 7.5 & & 30.0 & & & \\
\hline 1,1-Dichloroethene & 0.2105 & 0.2217 & 0.2092 & 0.2186 & 0.1957 & Ave & & 0.2111 & & & 0.0200 & 4.8 & & 30.0 & & & \\
\hline Acetone & 0.0124 & 0.0124 & 0.0100 & 0.0100 & 0.0092 & Ave & & 0.0108 & & & 0.0100 & 13.8 & & 40.0 & & & \\
\hline Carbon disulfide & 0.7855 & 0.6594 & 0.7311 & 0.5944 & 0.5415 & Ave & & 0.6624 & & & 0.0100 & 14.9 & & 20.0 & & & \\
\hline Methyl acetate & 0.0410 & 0.0416 & 0.0397 & 0.0386 & 0.0370 & Ave & & 0.0396 & & & 0.0100 & 4.6 & & 40.0 & & & \\
\hline Methylene Chloride & 0.2254 & 0.2243 & 0.2133 & 0.2165 & 0.1951 & Ave & & 0.2149 & & & 0.0100 & 5.7 & & 40.0 & & & \\
\hline Methyl tert-butyl ether & 0.2309 & 0.2517 & 0.2783 & 0.2885 & 0.2700 & Ave & & 0.2639 & & & 0.0100 & 8.7 & & 30.0 & & & \\
\hline trans-1,2-Dichloroethene & 0.3025 & 0.3169 & 0.3078 & 0.3250 & 0.2984 & Ave & & 0.3101 & & & 0.0700 & 3.5 & & 20.0 & & & \\
\hline 1,1-Dichloroethane & 0.5253 & 0.5151 & 0.5076 & 0.5271 & 0.4979 & Ave & & 0.5146 & & & 0.1000 & 2.4 & & 20.0 & & & \\
\hline cis-1,2-Dichloroethene & 0.2766 & 0.2808 & 0.2971 & 0.3103 & 0.2970 & Ave & & 0.2924 & & & 0.1000 & 4.7 & & 20.0 & & & \\
\hline 2-Butanone & 0.0212 & 0.0212 & 0.0234 & 0.0237 & 0.0220 & Ave & & 0.0223 & & & 0.0100 & 5.3 & & 40.0 & & & \\
\hline Bromochloromethane & 0.0992 & 0.0973 & 0.0980 & 0.0971 & 0.0925 & Ave & & 0.0968 & & & 0.0200 & 2.6 & & 20.0 & & & \\
\hline Chloroform & 0.5161 & 0.5022 & 0.4901 & 0.4971 & 0.4655 & Ave & & 0.4942 & & & 0.0400 & 3.8 & & 20.0 & & & \\
\hline 1,1,1-Trichloroethane & 0.7797 & 0.7611 & 0.7214 & 0.7538 & 0.7312 & Ave & & 0.7494 & & & 0.0500 & 3.1 & & 30.0 & & & \\
\hline Cyclohexane & 0.5772 & 0.6457 & 0.7093 & 0.7385 & 0.7338 & Ave & & 0.6809 & & & 0.1000 & 10.1 & & 30.0 & & & \\
\hline Carbon tetrachloride & 0.7512 & 0.7318 & 0.6965 & 0.7190 & 0.7100 & Ave & & 0.7217 & & & 0.0200 & 2.9 & & 20.0 & & & \\
\hline Benzene & 1.4678 & 1.4492 & 1.4851 & 1.5456 & 1.5004 & Ave & & 1.4896 & & & 0.3000 & 2.5 & & 20.0 & & & \\
\hline 1,2-Dichloroethane & 0.2219 & 0.2062 & 0.2145 & 0.2167 & 0.2006 & Ave & & 0.2120 & & & 0.0100 & 4.0 & & 20.0 & & & \\
\hline Trichloroethene & 0.4165 & 0.4213 & 0.4180 & 0.4319 & 0.4293 & Ave & & 0.4234 & & & 0.1000 & 1.6 & & 20.0 & & & \\
\hline Methylcyclohexane & 0.4697 & 0.5672 & 0.6186 & 0.6251 & 0.6088 & Ave & & 0.5779 & & & 0.2000 & 11.2 & & 30.0 & & & \\
\hline 1,2-Dichloropropane & 0.2911 & 0.2762 & 0.2865 & 0.2913 & 0.2903 & Ave & & 0.2871 & & & 0.1000 & $\frac{2.2}{2.2}$ & & 20.0 & & & \\
\hline Bromodichloromethane & 0.3738 & 0.3582 & 0.3531 & 0.3563 & 0.3516 & Ave & & 0.3586 & & & 0.0900 & 2.5 & & 20.0 & & & \\
\hline cis-1,3-Dichloropropene & 0.3243 & 0.3357 & 0.3898 & 0.4025 & 0.4002 & Ave & & 0.3705 & & & 0.1000 & 10.1 & & 20.0 & & & \\
\hline 4-Methyl-2-pentanone & 0.0587 & 0.0601 & 0.0701 & 0.0700 & 0.0670 & Ave & & 0.0652 & & & 0.0100 & 8.4 & & 30.0 & & & \\
\hline Toluene & 1.4137 & 1.5610 & 1.7069 & 1.7879 & 1.7088 & Ave & & 1.6357 & & & 0.4000 & 9.1 & & 20.0 & & & \\
\hline trans-1,3-Dichloropropene & 0.2311 & 0.2368 & 0.2737 & 0.2898 & 0.2892 & Ave & & 0.2641 & & & 0.0100 & 10.7 & & 30.0 & & & \\
\hline
\end{tabular}

Note: The MI coefficient is the same as Ave RRF for an Ave curve type.

FORM VI SOM02.2/VOA_Tr

Page 72 of 104 
FORM VI

GC/MS VOA BY INTERNAL STANDARD - INITIAL CALIBRATION DATA

CURVE EVALUATION

Lab Name: TestAmerica Burlington

Job No.: 200-38792-1

Analy Batch No.: 116931

SDG No.: 200-38792-1

Instrument ID: CHD.i

GC Column: DB-624

ID: $0.2(\mathrm{~mm})$

Heated Purge: (Y/N) N

Calibration Start Date: 05/23/2017 17:32

Calibration End Date: 05/23/2017 19:16

Calibration ID: 37252

\begin{tabular}{|c|c|c|c|c|c|c|c|c|c|c|c|c|c|c|c|c|c|}
\hline \multirow[t]{2}{*}{ ANALYTE } & \multicolumn{5}{|c|}{$\mathrm{RRF}$} & \multirow{2}{*}{$\begin{array}{l}\text { CURVE } \\
\text { TYPE }\end{array}$} & \multicolumn{3}{|c|}{ COEFFICIENT } & \multirow[t]{2}{*}{ \# } & \multirow[t]{2}{*}{ MIN RRF } & \multirow[t]{2}{*}{$\because \mathrm{RSD}$} & \multirow[t]{2}{*}{ \# } & \multirow{2}{*}{$\begin{array}{c}\text { MAX } \\
\because \mathrm{RSD}\end{array}$} & \multirow{2}{*}{$\begin{array}{c}R^{\wedge} 2 \\
O R \quad C O D\end{array}$} & \multirow[t]{2}{*}{ \# } & \multirow{2}{*}{$\begin{array}{ccc}\text { MIN } & R^{\wedge} 2 \\
\text { OR } & \text { COD }\end{array}$} \\
\hline & LVL 1 & LVL 2 & LVL 3 & LVL 4 & LVL 5 & & $\mathrm{~B}$ & M1 & M2 & & & & & & & & \\
\hline 1,1,2-Trichloroethane & 0.1549 & 0.1454 & 0.1452 & 0.1455 & 0.1463 & Ave & & 0.1475 & & & 0.0400 & 2.8 & & 20.0 & & & \\
\hline Tetrachloroethene & 0.4049 & 0.4469 & 0.4426 & 0.4660 & 0.4641 & Ave & & 0.4449 & & & 0.1000 & 5.5 & & 20.0 & & & \\
\hline 2-Hexanone & 0.0375 & 0.0423 & 0.0478 & 0.0482 & 0.0470 & Ave & & 0.0446 & & & 0.0100 & 10.3 & & 40.0 & & & \\
\hline Dibromochloromethane & 0.2073 & 0.2119 & 0.2209 & 0.2243 & 0.2283 & Ave & & 0.2185 & & & 0.0500 & 4.0 & & 20.0 & & & \\
\hline 1,2-Dibromoethane & 0.1227 & 0.1262 & 0.1295 & 0.1327 & 0.1347 & Ave & & 0.1292 & & & 0.0100 & 3.7 & & 20.0 & & & \\
\hline Chlorobenzene & 1.0578 & 1.0613 & 1.0665 & 1.0980 & 1.0702 & Ave & & 1.0707 & & & 0.4000 & 1.5 & & 20.0 & & & \\
\hline Ethylbenzene & 1.4947 & 1.7201 & 2.0033 & 2.1407 & 2.0712 & Ave & & 1.8860 & & & 0.5000 & 14.4 & & 20.0 & & & \\
\hline $\mathrm{m}, \mathrm{p}$-Xylene & 0.5785 & 0.6997 & 0.8212 & 0.8795 & 0.8501 & Ave & & 0.7658 & & & 0.2000 & 16.3 & & 20.0 & & & \\
\hline o-Xylene & 0.4978 & 0.5944 & 0.7343 & 0.7835 & 0.7566 & Ave & & 0.6733 & & & 0.3000 & 18.2 & & 30.0 & & & \\
\hline Styrene & 0.7553 & 0.9572 & 1.1189 & 1.1399 & 1.0857 & Ave & & 1.0114 & & & 0.2000 & 15.8 & & 30.0 & & & \\
\hline Bromoform & 0.2357 & 0.2370 & 0.2273 & 0.2321 & 0.2321 & Ave & & 0.2328 & & & 0.0100 & 1.6 & & 30.0 & & & \\
\hline Isopropylbenzene & 1.4003 & 1.7588 & 2.2312 & 2.4131 & 2.3137 & Ave & & 2.0234 & & & 0.7000 & 21.2 & & 30.0 & & & \\
\hline $1,1,2,2$-Tetrachloroethane & 0.1199 & 0.1243 & 0.1307 & 0.1323 & 0.1335 & Ave & & 0.1281 & & & 0.0500 & 4.5 & & 20.0 & & & \\
\hline 1,3-Dichlorobenzene & 1.5037 & 1.6899 & 1.7329 & 1.7806 & 1.7661 & Ave & & 1.6946 & & & 0.5000 & 6.6 & & 20.0 & & & \\
\hline 1,4-Dichlorobenzene & 1.8129 & 1.8842 & 1.7650 & 1.7319 & 1.6850 & Ave & & 1.7758 & & & 0.7000 & 4.3 & & 20.0 & & & \\
\hline 1,2-Dichlorobenzene & 1.2644 & 1.3583 & 1.3956 & 1.3788 & 1.3207 & Ave & & 1.3436 & & & 0.4000 & 3.9 & & 20.0 & & & \\
\hline 1,2-Dibromo-3-chloropropane & 0.0396 & 0.0380 & 0.0358 & 0.0353 & 0.0349 & Ave & & 0.0367 & & & 0.0100 & 5.4 & & 40.0 & & & \\
\hline 1,2,4-Trichlorobenzene & 0.6599 & 0.8382 & 0.7886 & 0.7901 & 0.7748 & Ave & & 0.7703 & & & 0.3000 & 8.6 & & 30.0 & & & \\
\hline 1,2,3-Trichlorobenzene & 0.4739 & 0.6165 & 0.5305 & 0.5208 & 0.4812 & Ave & & 0.5246 & & & 0.2000 & 10.8 & & 30.0 & & & \\
\hline Vinyl chloride-d3 & 0.1812 & 0.1897 & 0.1830 & 0.1994 & 0.1776 & Ave & & 0.1862 & & & 0.0100 & 4.6 & & 30.0 & & & \\
\hline Chloroethane-d5 & 0.1529 & 0.1477 & 0.1359 & 0.1367 & 0.1196 & Ave & & 0.1386 & & & 0.0100 & 9.3 & & 30.0 & & & \\
\hline 1,1-Dichloroethene-d2 & 0.4478 & 0.4855 & 0.4554 & 0.4679 & 0.4227 & Ave & & 0.4559 & & & 0.0100 & 5.1 & & 30.0 & & & \\
\hline 2-Butanone-d5 & 0.0221 & 0.0224 & 0.0236 & 0.0237 & 0.0223 & Ave & & 0.0228 & & & 0.0100 & 3.4 & & 40.0 & & & \\
\hline Chloroform-d & 0.5394 & 0.5317 & 0.5183 & 0.5256 & 0.4917 & Ave & & 0.5213 & & & 0.0100 & 3.5 & & 20.0 & & & \\
\hline 1,2-Dichloroethane-d4 & 0.1679 & 0.1638 & 0.1627 & 0.1639 & 0.1530 & Ave & & 0.1622 & & & 0.0100 & 3.4 & & 20.0 & & & \\
\hline Benzene-d6 & 1.4046 & 1.4263 & 1.4522 & 1.5093 & 1.4798 & Ave & & 1.4544 & & & 0.0300 & 2.9 & & 20.0 & & & \\
\hline 1,2-Dichloropropane-d6 & 0.3835 & 0.3975 & 0.3768 & 0.3660 & 0.3526 & Ave & & 0.3753 & & & 0.1000 & 4.5 & & 20.0 & & & \\
\hline Toluene-d8 & 1.2434 & 1.3295 & 1.4628 & 1.5376 & 1.4818 & Ave & & 1.4110 & & & 0.2000 & 8.6 & & 20.0 & & & \\
\hline trans-1,3-Dichloropropene-d4 & 0.2007 & 0.2044 & 0.2290 & 0.2484 & 0.2513 & Ave & & 0.2267 & & & 0.0100 & 10.5 & & 30.0 & & & \\
\hline 2-Hexanone-d5 & 0.0152 & 0.0173 & 0.0236 & 0.0252 & 0.0257 & Ave & & 0.0214 & & & 0.0100 & 22.6 & & 40.0 & & & \\
\hline $1,1,2,2$-Tetrachloroethane-d2 & 0.1292 & 0.1254 & 0.1300 & 0.1313 & 0.1315 & Ave & & 0.1295 & & & 0.0100 & 1.9 & & 20.0 & & & \\
\hline 1,2-Dichlorobenzene-d4 & 0.7910 & 0.8283 & 0.8270 & 0.8281 & 0.8024 & Ave & & 0.8154 & & & 0.0600 & 2.1 & & 20.0 & & & \\
\hline
\end{tabular}

Note: The M1 coefficient is the same as Ave RRF for an Ave curve type.

FORM VI SOM02.2/VOA_Tr

Page 73 of 104

06/30/2017 
FORM VI

GC/MS VOA BY INTERNAL STANDARD - INITIAL CALIBRATION DATA

RESPONSE AND CONCENTRATION

Lab Name: TestAmerica Burlington

Job No.: 200-38792-1

Analy Batch No.: 116931

SDG No.: 200-38792-1

Instrument ID: CHD.i

GC Column: DB-624

ID $: 0.2(\mathrm{~mm})$

Heated Purge: (Y/N) N

Calibration Start Date: 05/23/2017 17:32

Calibration End Date: 05/23/2017 19:16

Calibration ID: 37252

Calibration Files:
\begin{tabular}{|lll|}
\hline LEVEL: & LAB SAMPLE ID: & LAB FILE ID: \\
Level 1 & IC 200-116931/30 & 25203 11.D \\
Level 2 & IC 200-116931/29 & 25203 10.D \\
Level 3 & ICIS 200-116931/28 & 25203_09.D \\
Level 4 & IC 200-116931/27 & 25203_08.D \\
Level 5 & IC 200-116931/26 & 25203-07.D
\end{tabular}

Level 5 IC 200-116931/26 25203-07.D

\begin{tabular}{|c|c|c|c|c|c|c|c|c|c|c|c|c|}
\hline \multirow[t]{2}{*}{ ANALYTE } & \multirow{2}{*}{$\begin{array}{c}\text { IS } \\
\text { REF }\end{array}$} & \multirow{2}{*}{$\begin{array}{l}\text { CURVE } \\
\text { TYPE }\end{array}$} & \multicolumn{5}{|c|}{ RESPONSE } & \multicolumn{5}{|c|}{ CONCENTRATION (NG) } \\
\hline & & & LVL 1 & LVL 2 & LVL 3 & LVL 4 & LVL 5 & LVL 1 & LVL 2 & LVL 3 & LVL 4 & LVL 5 \\
\hline Dichlorodifluoromethane & DFBZ & Ave & 57634 & 124883 & 686599 & 1462880 & 2877321 & 12.5 & 25.0 & 125 & 250 & 500 \\
\hline Chloromethane & DFBZ & Ave & 51459 & 101847 & 486232 & 1060897 & 2054625 & 12.5 & 25.0 & 125 & 250 & 500 \\
\hline Vinyl chloride & DFBZ & Ave & 42926 & 90686 & 490522 & 1068986 & 2103582 & 12.5 & 25.0 & 125 & 250 & 500 \\
\hline Bromomethane & DFBZ & Ave & 19639 & 37820 & 202841 & 445933 & 849320 & 12.5 & 25.0 & 125 & 250 & 500 \\
\hline Chloroethane & DFBZ & Ave & 28430 & 53457 & 282653 & 597080 & 1140594 & 12.5 & 25.0 & 125 & 250 & 500 \\
\hline Trichlorofluoromethane & DFBZ & Ave & 94699 & 198075 & 1058461 & 2224907 & 4451094 & 12.5 & 25.0 & 125 & 250 & 500 \\
\hline $\begin{array}{l}\text { 1,1,2-Trichloro-1,2,2-trifluoroetha } \\
\text { ne }\end{array}$ & DFBZ & Ave & 44146 & 105934 & 545802 & 1120406 & 2197483 & 12.5 & 25.0 & 125 & 250 & 500 \\
\hline 1,1-Dichloroethene & DFBZ & Ave & 41042 & 86623 & 453129 & 954141 & 1863889 & 12.5 & 25.0 & 125 & 250 & 500 \\
\hline Acetone & DFBZ & Ave & 24094 & 48644 & 217369 & 437414 & 874615 & 125 & 250 & 1250 & 2499 & 4998 \\
\hline Carbon disulfide & DFBZ & Ave & 153169 & 257635 & 1583858 & 2593987 & 5157898 & 12.5 & 25.0 & 125 & 250 & 500 \\
\hline Methyl acetate & DFBZ & Ave & 7985 & 16240 & 86000 & 168518 & 352439 & 12.5 & 25.0 & 125 & 250 & 500 \\
\hline Methylene Chloride & DFBZ & Ave & 43942 & 87643 & 462126 & 944958 & 1858337 & 12.5 & 25.0 & 125 & 250 & 500 \\
\hline Methyl tert-butyl ether & DFBZ & Ave & 45018 & 98337 & 602822 & 1258874 & 2572172 & 12.5 & 25.0 & 125 & 250 & 500 \\
\hline trans-1,2-Dichloroethene & DFBZ & Ave & 58976 & 123809 & 666692 & 1418479 & 2842676 & 12.5 & 25.0 & 125 & 250 & 500 \\
\hline 1,1-Dichloroethane & DFBZ & Ave & 102433 & 201287 & 1099593 & 2300411 & 4742846 & 12.5 & 25.0 & 125 & 250 & 500 \\
\hline cis-1,2-Dichloroethene & DFBZ & Ave & 53940 & 109700 & 643547 & 1354226 & 2828871 & 12.5 & 25.0 & 125 & 250 & 500 \\
\hline 2-Butanone & DFBZ & Ave & 41365 & 82803 & 507396 & 1033103 & 2098434 & 125 & 250 & 1250 & 2499 & 4998 \\
\hline Bromochloromethane & DFBZ & Ave & 19336 & 38015 & 212204 & 423593 & 881268 & 12.5 & 25.0 & 125 & 250 & 500 \\
\hline Chloroform & DFBZ & Ave & 100639 & 196226 & 1061678 & 2169218 & 4434137 & 12.5 & 25.0 & 125 & 250 & 500 \\
\hline 1,1,1-Trichloroethane & CBNZd & Ave & 112909 & 229158 & 1228544 & 2621521 & 5418710 & 12.5 & 25.0 & 125 & 250 & 500 \\
\hline Cyclohexane & CBNZd & Ave & 83575 & 194413 & 1208029 & 2568285 & 5437754 & 12.5 & 25.0 & 125 & 250 & 500 \\
\hline Carbon tetrachloride & CBNZd & Ave & 108777 & 220339 & 1186237 & 2500463 & 5261594 & 12.5 & 25.0 & 125 & 250 & 500 \\
\hline Benzene & CBNZd & Ave & 212541 & 436354 & 2529209 & 5375362 & 11118807 & 12.5 & 25.0 & 125 & 250 & 500 \\
\hline 1,2-Dichloroethane & DFBZ & Ave & 43263 & 80577 & 464650 & 945606 & 1911142 & 12.5 & 25.0 & 125 & 250 & 500 \\
\hline Trichloroethene & CBNZd & Ave & 60305 & 126855 & 711918 & 1502147 & 3181258 & 12.5 & 25.0 & 125 & 250 & 500 \\
\hline Methylcyclohexane & CBNZd & Ave & 68019 & 170788 & 1053463 & 2173991 & 4511913 & 12.5 & 25.0 & 125 & 250 & 500 \\
\hline 1,2-Dichloropropane & CBNZd & Ave & 42150 & 83177 & 487872 & 1012998 & 2151195 & 12.5 & 25.0 & 125 & 250 & 500 \\
\hline Bromodichloromethane & CBNZd & Ave & 54124 & 107860 & 601365 & 1239271 & 2605524 & 12.5 & 25.0 & 125 & 250 & 500 \\
\hline cis-1,3-Dichloropropene & CBNZd & Ave & 46954 & 101090 & 663862 & 1399701 & 2965752 & 12.5 & 25.0 & 125 & 250 & 500 \\
\hline 4-Methyl-2-pentanone & CBNZd & Ave & 84969 & 180956 & 1193194 & 2434244 & 4968598 & 125 & 250 & 1250 & 2499 & 4998 \\
\hline Toluene & CBNZd & Ave & 204707 & 470020 & 2906868 & 6217956 & 12663711 & 12.5 & 25.0 & 125 & 250 & 500 \\
\hline trans-1,3-Dichloropropene & CBNZd & Ave & 33457 & 71298 & 466198 & 1007971 & 2142892 & 12.5 & 25.0 & 125 & 250 & 500 \\
\hline 1,1,2-Trichloroethane & CBNZd & Ave & 22425 & 43781 & 247232 & 506027 & 1084498 & 12.5 & 25.0 & 125 & 250 & 500 \\
\hline
\end{tabular}


FORM VI

GC/MS VOA BY INTERNAL STANDARD - INITIAL CALIBRATION DATA

RESPONSE AND CONCENTRATION

Lab Name: TestAmerica Burlington

Job No.: 200-38792-1

Analy Batch No.: 116931

SDG No.: 200-38792-1

Instrument ID: CHD.i

GC Column: DB-624

ID: $0.2(\mathrm{~mm})$

Heated Purge: (Y/N) N

Calibration Start Date: 05/23/2017 17:32

Calibration End Date: 05/23/2017 19:16

Calibration ID: 37252

\begin{tabular}{|c|c|c|c|c|c|c|c|c|c|c|c|c|}
\hline \multirow[t]{2}{*}{ ANALYTE } & \multirow{2}{*}{$\begin{array}{c}\text { IS } \\
\text { REF }\end{array}$} & \multirow{2}{*}{$\begin{array}{l}\text { CURVE } \\
\text { TYPE }\end{array}$} & \multicolumn{5}{|c|}{ RESPONSE } & \multicolumn{5}{|c|}{ CONCENTRATION (NG) } \\
\hline & & & LVL 1 & LVL 2 & LVL 3 & LVL 4 & LVL 5 & LVL 1 & LVL 2 & LVL 3 & LVL 4 & LVL 5 \\
\hline Tetrachloroethene & CBNZd & Ave & 58633 & 134557 & 753708 & 1620604 & 3439198 & 12.5 & 25.0 & 125 & 250 & 500 \\
\hline 2-Hexanone & CBNZd & Ave & 54323 & 127341 & 813459 & 1676963 & 3482711 & 125 & 250 & 1250 & 2499 & 4998 \\
\hline Dibromochloromethane & CBNZd & Ave & 30014 & 63799 & 376181 & 780061 & 1692129 & 12.5 & 25.0 & 125 & 250 & 500 \\
\hline 1,2-Dibromoethane & CBNZd & Ave & 17774 & 37992 & 220618 & 461387 & 997918 & 12.5 & 25.0 & 125 & 250 & 500 \\
\hline Chlorobenzene & CBNZd & Ave & 153171 & 319540 & 1816219 & 3818780 & 7930922 & 12.5 & 25.0 & 125 & 250 & 500 \\
\hline Ethylbenzene & CBNZd & Ave & 216443 & 517907 & 3411734 & 7444910 & 15349320 & 12.5 & 25.0 & 125 & 250 & 500 \\
\hline m,p-Xylene & CBNZd & Ave & 83770 & 210685 & 1398467 & 3058666 & 6299841 & 12.5 & 25.0 & 125 & 250 & 500 \\
\hline o-Xylene & CBNZd & Ave & 72088 & 178967 & 1250568 & 2724872 & 5606651 & 12.5 & 25.0 & 125 & 250 & 500 \\
\hline Styrene & CBNZd & Ave & 109371 & 288212 & 1905521 & 3964400 & 8046243 & 12.5 & 25.0 & 125 & 250 & 500 \\
\hline Bromoform & DCBd4 & Ave & 14545 & 31435 & 185943 & 407080 & 875820 & 12.5 & 25.0 & 125 & 250 & 500 \\
\hline Isopropylbenzene & CBNZd & Ave & 202769 & 529579 & 3799890 & 8392182 & 17146042 & 12.5 & 25.0 & 125 & 250 & 500 \\
\hline $1,1,2,2$-Tetrachloroethane & CBNZd & Ave & 17355 & 37433 & 222561 & 459988 & 989656 & 12.5 & 25.0 & 125 & 250 & 500 \\
\hline 1,3-Dichlorobenzene & DCBd4 & Ave & 92797 & 224144 & 1417560 & 3122441 & 6663707 & 12.5 & 25.0 & 125 & 250 & 500 \\
\hline 1,4-Dichlorobenzene & DCBd4 & Ave & 111879 & 249908 & 1443872 & 3037086 & 6357557 & 12.5 & 25.0 & 125 & 250 & 500 \\
\hline 1,2-Dichlorobenzene & DCBd4 & Ave & 78033 & 180159 & 1141663 & 2417944 & 4983299 & 12.5 & 25.0 & 125 & 250 & 500 \\
\hline 1,2-Dibromo-3-chloropropane & DCBd4 & Ave & 2445 & 5035 & 29323 & 61926 & 131733 & 12.5 & 25.0 & 125 & 250 & 500 \\
\hline 1,2,4-Trichlorobenzene & DCBd4 & Ave & 40727 & 111170 & 645152 & 1385442 & 2923279 & 12.5 & 25.0 & 125 & 250 & 500 \\
\hline 1,2,3-Trichlorobenzene & DCBd4 & Ave & 29244 & 81772 & 433951 & 913282 & 1815726 & 12.5 & 25.0 & 125 & 250 & 500 \\
\hline Vinyl chloride-d3 & DFBZ & Ave & 35337 & 74132 & 396467 & 870286 & 1691603 & 12.5 & 25.0 & 125 & 250 & 500 \\
\hline Chloroethane-d5 & DFBZ & Ave & 29812 & 57724 & 294382 & 596534 & 1138793 & 12.5 & 25.0 & 125 & 250 & 500 \\
\hline 1,1-Dichloroethene-d2 & DFBZ & Ave & 87317 & 189701 & 986550 & 2041886 & 4026296 & 12.5 & 25.0 & 125 & 250 & 500 \\
\hline 2-Butanone-d5 & DFBZ & Ave & 43121 & 87438 & 510872 & 1035798 & 2126763 & 125 & 250 & 1250 & 2499 & 4998 \\
\hline Chloroform-d & DFBZ & Ave & 105177 & 207761 & 1122856 & 2293637 & 4683779 & 12.5 & 25.0 & 125 & 250 & 500 \\
\hline 1,2-Dichloroethane-d4 & DFBZ & Ave & 32739 & 64001 & 352432 & 715186 & 1457034 & 12.5 & 25.0 & 125 & 250 & 500 \\
\hline Benzene-d6 & CBNZd & Ave & 203395 & 429457 & 2473159 & 5249113 & 10966290 & 12.5 & 25.0 & 125 & 250 & 500 \\
\hline 1,2-Dichloropropane-d6 & CBNZd & Ave & 55537 & 119677 & 641718 & 1272716 & 2613161 & 12.5 & 25.0 & 125 & 250 & 500 \\
\hline Toluene-d8 & CBNZd & Ave & 180051 & 400296 & 2491150 & 5347415 & 10981321 & 12.5 & 25.0 & 125 & 250 & 500 \\
\hline trans-1,3-Dichloropropene-d4 & CBNZd & Ave & 29063 & 61548 & 389973 & 863783 & 1862005 & 12.5 & 25.0 & 125 & 250 & 500 \\
\hline 2-Hexanone-d5 & CBNZd & Ave & 21997 & 52100 & 401406 & 878130 & 1906833 & 125 & 250 & 1250 & 2499 & 4998 \\
\hline $1,1,2,2$-Tetrachloroethane-d2 & CBNZd & Ave & 18705 & 37760 & 221440 & 456691 & 974885 & 12.5 & 25.0 & 125 & 250 & 500 \\
\hline 1,2-Dichlorobenzene-d4 & DCBd4 & Ave & 48819 & 109863 & 676513 & 1452166 & 3027550 & 12.5 & 25.0 & 125 & 250 & 500 \\
\hline
\end{tabular}

Curve Type Legend:

Ave = Average ISTD 
FORM VII

GC/MS VOA CONTINUING CALIBRATION DATA

Lab Name: TestAmerica Burlington

SDG No.: 200-38792-1

Lab Sample ID: ICV 200-116931/31

Instrument ID: CHD.i

GC Column: DB-624

ID $: 0.20(\mathrm{~mm})$

Lab File ID: 25203_12.D

EPA Sample No.: VICVEA
Job No.: 200-38792-1

Calibration Date: 05/23/2017 19:42

Calib Start Date: 05/23/2017 17:32

Calib End Date: 05/23/2017 19:16

Conc. Units: ug/L Heated Purge: (Y/N) N

\begin{tabular}{|c|c|c|c|c|c|c|c|c|}
\hline ANALYTE & $\begin{array}{c}\text { CURVE } \\
\text { TYPE }\end{array}$ & AVE RRF & $\mathrm{RRF}$ & MIN RRF & $\begin{array}{c}\text { CALC } \\
\text { AMOUNT }\end{array}$ & $\begin{array}{c}\text { SPIKE } \\
\text { AMOUNT }\end{array}$ & $\because \mathrm{D}$ & $\begin{array}{l}\mathrm{MAX} \\
\frac{\circ}{\circ}\end{array}$ \\
\hline Dichlorodifluoromethane & Ave & 0.3139 & 0.2080 & 0.0100 & 3.30 & 5.00 & -33.7 & 40.0 \\
\hline Chloromethane & Ave & 0.2416 & 0.1762 & 0.0100 & 3.60 & 5.00 & -27.1 & 30.0 \\
\hline Vinyl chloride & Ave & 0.2289 & 0.1880 & 0.0100 & 4.10 & 5.00 & -17.9 & 30.0 \\
\hline Bromomethane & Ave & 0.0965 & 0.1107 & 0.0100 & 5.70 & 5.00 & 14.7 & 30.0 \\
\hline Chloroethane & Ave & 0.1339 & 0.1210 & 0.0100 & 4.50 & 5.00 & -9.7 & 30.0 \\
\hline Trichlorofluoromethane & Ave & 0.4917 & 0.4553 & 0.0100 & 4.60 & 5.00 & -7.4 & 30.0 \\
\hline $\begin{array}{l}1,1,2 \text {-Trichloro-1,2,2-triflu } \\
\text { oroethane }\end{array}$ & Ave & 0.2474 & 0.2481 & 0.0100 & 5.00 & 5.00 & 0.3 & 30.0 \\
\hline 1,1-Dichloroethene & Ave & 0.2111 & 0.2124 & 0.0200 & 5.00 & 5.00 & 0.6 & 20.0 \\
\hline Acetone & Ave & 0.0108 & $0.0097 *$ & 0.0100 & 45.0 & 50.0 & -10.1 & 40.0 \\
\hline Carbon disulfide & Ave & 0.6624 & 0.6143 & 0.0100 & 4.60 & 5.00 & -7.3 & 25.0 \\
\hline Methyl acetate & Ave & 0.0396 & 0.0389 & 0.0100 & 25.0 & 25.0 & -1.7 & 40.0 \\
\hline Methylene Chloride & Ave & 0.2149 & 0.2049 & 0.0100 & 4.80 & 5.00 & -4.6 & 30.0 \\
\hline Methyl tert-butyl ether & Ave & 0.2639 & 0.2623 & 0.0100 & 5.00 & 5.00 & -0.6 & 30.0 \\
\hline trans-1,2-Dichloroethene & Ave & 0.3101 & 0.3046 & 0.0700 & 4.90 & 5.00 & -1.8 & 20.0 \\
\hline 1,1-Dichloroethane & Ave & 0.5146 & 0.4937 & 0.1000 & 4.80 & 5.00 & -4.1 & 20.0 \\
\hline cis-1,2-Dichloroethene & Ave & 0.2924 & 0.2891 & 0.1000 & 4.90 & 5.00 & -1.1 & 20.0 \\
\hline 2-Butanone & Ave & 0.0223 & 0.0231 & 0.0100 & 52.0 & 50.0 & 3.6 & 40.0 \\
\hline Bromochloromethane & Ave & 0.0968 & 0.0888 & 0.0200 & 4.60 & 5.00 & -8.2 & 20.0 \\
\hline Chloroform & Ave & 0.4942 & 0.4676 & 0.0400 & 4.70 & 5.00 & -5.4 & 20.0 \\
\hline $1,1,1$-Trichloroethane & Ave & 0.7494 & 0.7567 & 0.0500 & 5.00 & 5.00 & 1.0 & 20.0 \\
\hline Cyclohexane & Ave & 0.6809 & 0.7365 & 0.1000 & 5.40 & 5.00 & 8.2 & 25.0 \\
\hline Carbon tetrachloride & Ave & 0.7217 & 0.7304 & 0.0200 & 5.10 & 5.00 & 1.2 & 25.0 \\
\hline Benzene & Ave & 1.490 & 1.609 & 0.3000 & 5.40 & 5.00 & 8.0 & 20.0 \\
\hline 1,2-Dichloroethane & Ave & 0.2120 & 0.2009 & 0.0100 & 4.70 & 5.00 & -5.2 & 25.0 \\
\hline Trichloroethene & Ave & 0.4234 & 0.4221 & 0.1000 & 5.00 & 5.00 & -0.3 & 20.0 \\
\hline Methylcyclohexane & Ave & 0.5779 & 0.6086 & 0.2000 & 5.30 & 5.00 & 5.3 & 25.0 \\
\hline 1,2-Dichloropropane & Ave & 0.2871 & 0.2902 & 0.1000 & 5.10 & 5.00 & 1.1 & 20.0 \\
\hline Bromodichloromethane & Ave & 0.3586 & 0.3565 & 0.0900 & 5.00 & 5.00 & -0.6 & 20.0 \\
\hline cis-1,3-Dichloropropene & Ave & 0.3705 & 0.3852 & 0.1000 & 5.20 & 5.00 & 4.0 & 20.0 \\
\hline 4-Methyl-2-pentanone & Ave & 0.0652 & 0.0726 & 0.0100 & 56.0 & 50.0 & 11.4 & 30.0 \\
\hline Toluene & Ave & 1.636 & 1.791 & 0.4000 & 5.50 & 5.00 & 9.5 & 20.0 \\
\hline trans-1,3-Dichloropropene & Ave & 0.2641 & 0.2649 & 0.0100 & 5.00 & 5.00 & 0.3 & 20.0 \\
\hline $1,1,2$-Trichloroethane & Ave & 0.1475 & 0.1505 & 0.0400 & 5.10 & 5.00 & 2.0 & 20.0 \\
\hline Tetrachloroethene & Ave & 0.4449 & 0.4449 & 0.1000 & 5.00 & 5.00 & 0.0 & 20.0 \\
\hline 2-Hexanone & Ave & 0.0446 & 0.0498 & 0.0100 & 56.0 & 50.0 & 11.7 & 40.0 \\
\hline Dibromochloromethane & Ave & 0.2185 & 0.2183 & 0.0500 & 5.00 & 5.00 & -0.1 & 20.0 \\
\hline 1,2-Dibromoethane & Ave & 0.1292 & 0.1293 & 0.0100 & 5.00 & 5.00 & 0.1 & 20.0 \\
\hline Chlorobenzene & Ave & 1.071 & 1.072 & 0.4000 & 5.00 & 5.00 & 0.1 & 20.0 \\
\hline Ethylbenzene & Ave & 1.886 & 2.060 & 0.5000 & 5.50 & 5.00 & 9.2 & 20.0 \\
\hline
\end{tabular}

FORM VII SOM02.2/VOA_Tr 
FORM VII

GC/MS VOA CONTINUING CALIBRATION DATA

Lab Name: TestAmerica Burlington

SDG No.: 200-38792-1

Lab Sample ID: ICV 200-116931/31

Instrument ID: CHD.i

GC Column: DB-624

Lab File ID: 25203 12.D

EPA Sample No.: VICVEA
Job No.: 200-38792-1

Calibration Date: 05/23/2017 19:42

Calib Start Date: 05/23/2017 17:32

Calib End Date: 05/23/2017 19:16

Conc. Units: ug/L Heated Purge: (Y/N) N

\begin{tabular}{|c|c|c|c|c|c|c|c|c|}
\hline ANALYTE & $\begin{array}{c}\text { CURVE } \\
\text { TYPE }\end{array}$ & AVE RRF & $\mathrm{RRF}$ & MIN RRF & $\begin{array}{c}\text { CALC } \\
\text { AMOUNT }\end{array}$ & $\begin{array}{c}\text { SPIKE } \\
\text { AMOUNT }\end{array}$ & $\div D$ & $\begin{array}{l}\text { MAX } \\
\because D\end{array}$ \\
\hline $\mathrm{m}, \mathrm{p}$-Xylene & Ave & 0.7658 & 0.8445 & 0.2000 & 5.50 & 5.00 & 10.3 & 20.0 \\
\hline o-Xylene & Ave & 0.6733 & 0.7558 & 0.3000 & 5.60 & 5.00 & 12.2 & 20.0 \\
\hline Styrene & Ave & 1.011 & 1.131 & 0.2000 & 5.60 & 5.00 & 11.8 & 20.0 \\
\hline Bromoform & Ave & 0.2328 & 0.1899 & 0.0100 & 4.10 & 5.00 & -18.5 & 30.0 \\
\hline Isopropylbenzene & Ave & 2.023 & 2.287 & 0.7000 & 5.60 & 5.00 & 13.0 & 25.0 \\
\hline $1,1,2,2$-Tetrachloroethane & Ave & 0.1281 & 0.1384 & 0.0500 & 5.40 & 5.00 & 8.0 & 25.0 \\
\hline 1,3-Dichlorobenzene & Ave & 1.695 & 1.642 & 0.5000 & 4.80 & 5.00 & -3.1 & 20.0 \\
\hline 1,4-Dichlorobenzene & Ave & 1.776 & 1.607 & 0.7000 & 4.50 & 5.00 & -9.5 & 20.0 \\
\hline 1,2-Dichlorobenzene & Ave & 1.344 & 1.292 & 0.4000 & 4.80 & 5.00 & -3.9 & 20.0 \\
\hline 1,2-Dibromo-3-chloropropane & Ave & 0.0367 & 0.0337 & 0.0100 & 4.60 & 5.00 & -8.3 & 40.0 \\
\hline 1,2,4-Trichlorobenzene & Ave & 0.7703 & 0.6470 & 0.3000 & 4.20 & 5.00 & -16.0 & 30.0 \\
\hline 1,2,3-Trichlorobenzene & Ave & 0.5246 & 0.4285 & 0.2000 & 4.10 & 5.00 & -18.3 & 40.0 \\
\hline Vinyl chloride-d3 & Ave & 0.1862 & 0.1772 & 0.0100 & 4.80 & 5.00 & -4.8 & 30.0 \\
\hline Chloroethane-d5 & Ave & 0.1386 & 0.1338 & 0.0100 & 4.80 & 5.00 & -3.4 & 30.0 \\
\hline 1,1-Dichloroethene-d2 & Ave & 0.4559 & 0.4417 & 0.0100 & 4.80 & 5.00 & -3.1 & 25.0 \\
\hline 2-Butanone-d5 & Ave & 0.0228 & 0.0223 & 0.0100 & 49.0 & 50.0 & -2.3 & 40.0 \\
\hline Chloroform-d & Ave & 0.5213 & 0.5046 & 0.0100 & 4.80 & 5.00 & -3.2 & 20.0 \\
\hline 1,2-Dichloroethane-d4 & Ave & 0.1622 & 0.1620 & 0.0100 & 5.00 & 5.00 & -0.2 & 25.0 \\
\hline Benzene-d6 & Ave & 1.454 & 1.597 & 0.0300 & 5.50 & 5.00 & 9.8 & 20.0 \\
\hline 1,2-Dichloropropane-d6 & Ave & 0.3753 & 0.3879 & 0.1000 & 5.20 & 5.00 & 3.4 & 20.0 \\
\hline Toluene-d8 & Ave & 1.411 & 1.545 & 0.2000 & 5.50 & 5.00 & 9.5 & 20.0 \\
\hline trans-1,3-Dichloropropene-d4 & Ave & 0.2267 & 0.2312 & 0.0100 & 5.10 & 5.00 & 2.0 & 25.0 \\
\hline 2-Hexanone-d5 & Ave & 0.0214 & 0.0253 & 0.0100 & 59.0 & 50.0 & 18.2 & 40.0 \\
\hline $1,1,2,2$-Tetrachloroethane-d2 & Ave & 0.1295 & 0.1358 & 0.0100 & 5.20 & 5.00 & 4.9 & 25.0 \\
\hline 1,2-Dichlorobenzene-d4 & Ave & 0.8154 & 0.7982 & 0.0600 & 4.90 & 5.00 & -2.1 & 20.0 \\
\hline
\end{tabular}


FORM VII

GC/MS VOA CONTINUING CALIBRATION DATA

Lab Name: TestAmerica Burlington

SDG No.: 200-38792-1

Lab Sample ID: CCVIS 200-117296/6

Instrument ID: CHD.i

GC Column: DB-624

ID: $0.20(\mathrm{~mm})$

Lab File ID: 25366_06.D

EPA Sample No.: VSTD005ET
Job No.: 200-38792-1

Calibration Date: 06/06/2017 15:17

Calib Start Date: 05/23/2017 17:32

Calib End Date: 05/23/2017 19:16

Conc. Units: ug/L Heated Purge: (Y/N) N

\begin{tabular}{|c|c|c|c|c|c|c|c|c|}
\hline ANALYTE & $\begin{array}{c}\text { CURVE } \\
\text { TYPE }\end{array}$ & AVE RRF & $\mathrm{RRF}$ & MIN RRF & $\begin{array}{c}\text { CALC } \\
\text { AMOUNT }\end{array}$ & $\begin{array}{c}\text { SPIKE } \\
\text { AMOUNT }\end{array}$ & $\div D$ & $\begin{array}{l}\mathrm{MAX} \\
\frac{\circ}{\circ}\end{array}$ \\
\hline Dichlorodifluoromethane & Ave & 0.3139 & 0.2658 & 0.0100 & 4.20 & 5.00 & -15.3 & 40.0 \\
\hline Chloromethane & Ave & 0.2416 & 0.2330 & 0.0100 & 4.80 & 5.00 & -3.5 & 30.0 \\
\hline Vinyl chloride & Ave & 0.2289 & 0.2376 & 0.0100 & 5.20 & 5.00 & 3.8 & 30.0 \\
\hline Bromomethane & Ave & 0.0965 & 0.0904 & 0.0100 & 4.70 & 5.00 & -6.3 & 30.0 \\
\hline Chloroethane & Ave & 0.1339 & 0.1529 & 0.0100 & 5.70 & 5.00 & 14.2 & 30.0 \\
\hline Trichlorofluoromethane & Ave & 0.4917 & 0.5481 & 0.0100 & 5.60 & 5.00 & 11.5 & 30.0 \\
\hline $\begin{array}{l}1,1,2 \text {-Trichloro-1,2,2-triflu } \\
\text { oroethane }\end{array}$ & Ave & 0.2474 & 0.2843 & 0.0100 & 5.70 & 5.00 & 14.9 & 30.0 \\
\hline 1,1-Dichloroethene & Ave & 0.2111 & 0.2354 & 0.0200 & 5.60 & 5.00 & 11.5 & 20.0 \\
\hline Acetone & Ave & 0.0108 & 0.0116 & 0.0100 & 53.0 & 50.0 & 6.9 & 40.0 \\
\hline Carbon disulfide & Ave & 0.6624 & 0.5996 & 0.0100 & 4.50 & 5.00 & -9.5 & 25.0 \\
\hline Methyl acetate & Ave & 0.0396 & 0.0368 & 0.0100 & 4.60 & 5.00 & -7.1 & 40.0 \\
\hline Methylene Chloride & Ave & 0.2149 & 0.2385 & 0.0100 & 5.50 & 5.00 & 11.0 & 30.0 \\
\hline Methyl tert-butyl ether & Ave & 0.2639 & 0.3005 & 0.0100 & 5.70 & 5.00 & 13.9 & 30.0 \\
\hline trans-1,2-Dichloroethene & Ave & 0.3101 & 0.3341 & 0.0700 & 5.40 & 5.00 & 7.7 & 20.0 \\
\hline 1,1-Dichloroethane & Ave & 0.5146 & 0.5786 & 0.1000 & 5.60 & 5.00 & 12.4 & 20.0 \\
\hline cis-1,2-Dichloroethene & Ave & 0.2924 & 0.3286 & 0.1000 & 5.60 & 5.00 & 12.4 & 20.0 \\
\hline 2-Butanone & Ave & 0.0223 & 0.0252 & 0.0100 & 56.0 & 50.0 & 13.0 & 40.0 \\
\hline Bromochloromethane & Ave & 0.0968 & 0.1093 & 0.0200 & 5.60 & 5.00 & 12.9 & 20.0 \\
\hline Chloroform & Ave & 0.4942 & 0.5686 & 0.0400 & 5.80 & 5.00 & 15.1 & 20.0 \\
\hline $1,1,1$-Trichloroethane & Ave & 0.7494 & 0.7802 & 0.0500 & 5.20 & 5.00 & 4.1 & 20.0 \\
\hline Cyclohexane & Ave & 0.6809 & 0.7062 & 0.1000 & 5.20 & 5.00 & 3.7 & 25.0 \\
\hline Carbon tetrachloride & Ave & 0.7217 & 0.7535 & 0.0200 & 5.20 & 5.00 & 4.4 & 25.0 \\
\hline Benzene & Ave & 1.490 & 1.603 & 0.3000 & 5.40 & 5.00 & 7.6 & 20.0 \\
\hline 1,2-Dichloroethane & Ave & 0.2120 & 0.2589 & 0.0100 & 6.10 & 5.00 & 22.1 & 25.0 \\
\hline Trichloroethene & Ave & 0.4234 & 0.4266 & 0.1000 & 5.00 & 5.00 & 0.8 & 20.0 \\
\hline Methylcyclohexane & Ave & 0.5779 & 0.6335 & 0.2000 & 5.50 & 5.00 & 9.6 & 25.0 \\
\hline 1,2-Dichloropropane & Ave & 0.2871 & 0.3129 & 0.1000 & 5.40 & 5.00 & 9.0 & 20.0 \\
\hline Bromodichloromethane & Ave & 0.3586 & 0.3887 & 0.0900 & 5.40 & 5.00 & 8.4 & 20.0 \\
\hline cis-1,3-Dichloropropene & Ave & 0.3705 & 0.4240 & 0.1000 & 5.70 & 5.00 & 14.4 & 20.0 \\
\hline 4-Methyl-2-pentanone & Ave & 0.0652 & 0.0758 & 0.0100 & 58.0 & 50.0 & 16.2 & 30.0 \\
\hline Toluene & Ave & 1.636 & 1.856 & 0.4000 & 5.70 & 5.00 & 13.4 & 20.0 \\
\hline trans-1,3-Dichloropropene & Ave & 0.2641 & 0.2998 & 0.0100 & 5.70 & 5.00 & 13.5 & 20.0 \\
\hline $1,1,2$-Trichloroethane & Ave & 0.1475 & 0.1587 & 0.0400 & 5.40 & 5.00 & 7.6 & 20.0 \\
\hline Tetrachloroethene & Ave & 0.4449 & 0.4563 & 0.1000 & 5.10 & 5.00 & 2.6 & 20.0 \\
\hline 2-Hexanone & Ave & 0.0446 & 0.0517 & 0.0100 & 58.0 & 50.0 & 16.1 & 40.0 \\
\hline Dibromochloromethane & Ave & 0.2185 & 0.2418 & 0.0500 & 5.50 & 5.00 & 10.6 & 20.0 \\
\hline 1,2-Dibromoethane & Ave & 0.1292 & 0.1405 & 0.0100 & 5.40 & 5.00 & 8.8 & 20.0 \\
\hline Chlorobenzene & Ave & 1.071 & 1.156 & 0.4000 & 5.40 & 5.00 & 7.9 & 20.0 \\
\hline Ethylbenzene & Ave & 1.886 & 2.158 & 0.5000 & 5.70 & 5.00 & 14.4 & 20.0 \\
\hline
\end{tabular}

FORM VII SOM02.2/VOA_Tr 
FORM VII

GC/MS VOA CONTINUING CALIBRATION DATA

Lab Name: TestAmerica Burlington

SDG No.: 200-38792-1

Lab Sample ID: CCVIS 200-117296/6

Instrument ID: CHD.i

GC Column: DB-624

ID $: 0.20(\mathrm{~mm})$

Lab File ID: 25366 06.D

EPA Sample No.: VSTD005ET
Job No.: 200-38792-1

Calibration Date: 06/06/2017 15:17

Calib Start Date: 05/23/2017 17:32

Calib End Date: 05/23/2017 19:16

Conc. Units: ug/L Heated Purge: (Y/N) N

\begin{tabular}{|c|c|c|c|c|c|c|c|c|}
\hline ANALYTE & $\begin{array}{l}\text { CURVE } \\
\text { TYPE }\end{array}$ & AVE RRF & RRF & MIN RRF & $\begin{array}{c}\text { CALC } \\
\text { AMOUNT }\end{array}$ & $\begin{array}{c}\text { SPIKE } \\
\text { AMOUNT }\end{array}$ & $\div D$ & $\begin{array}{l}\mathrm{MAX} \\
\div \mathrm{D}\end{array}$ \\
\hline $\mathrm{m}, \mathrm{p}$-Xylene & Ave & 0.7658 & 0.8887 & 0.2000 & 5.80 & 5.00 & 16.0 & 20.0 \\
\hline o-Xylene & Ave & 0.6733 & 0.7944 & 0.3000 & 5.90 & 5.00 & 18.0 & 20.0 \\
\hline Styrene & Ave & 1.011 & 1.218 & 0.2000 & 6.00 & 5.00 & $20.4 *$ & 20.0 \\
\hline Bromoform & Ave & 0.2328 & 0.2355 & 0.0100 & 5.10 & 5.00 & 1.1 & 30.0 \\
\hline Isopropylbenzene & Ave & 2.023 & 2.377 & 0.7000 & 5.90 & 5.00 & 17.5 & 25.0 \\
\hline $1,1,2,2$-Tetrachloroethane & Ave & 0.1281 & 0.1441 & 0.0500 & 5.60 & 5.00 & 12.4 & 25.0 \\
\hline 1,3-Dichlorobenzene & Ave & 1.695 & 1.641 & 0.5000 & 4.80 & 5.00 & -3.1 & 20.0 \\
\hline 1,4-Dichlorobenzene & Ave & 1.776 & 1.722 & 0.7000 & 4.80 & 5.00 & -3.0 & 20.0 \\
\hline 1,2-Dichlorobenzene & Ave & 1.344 & 1.359 & 0.4000 & 5.10 & 5.00 & 1.1 & 20.0 \\
\hline 1,2-Dibromo-3-chloropropane & Ave & 0.0367 & 0.0346 & 0.0100 & 4.70 & 5.00 & -5.8 & 40.0 \\
\hline 1,2,4-Trichlorobenzene & Ave & 0.7703 & 0.6182 & 0.3000 & 4.00 & 5.00 & -19.7 & 30.0 \\
\hline 1,2,3-Trichlorobenzene & Ave & 0.5246 & 0.3852 & 0.2000 & 3.70 & 5.00 & -26.6 & 40.0 \\
\hline Vinyl chloride-d3 & Ave & 0.1862 & 0.1933 & 0.0100 & 5.20 & 5.00 & 3.8 & 30.0 \\
\hline Chloroethane-d5 & Ave & 0.1386 & 0.1533 & 0.0100 & 5.50 & 5.00 & 10.7 & 30.0 \\
\hline 1,1-Dichloroethene-d2 & Ave & 0.4559 & 0.5189 & 0.0100 & 5.70 & 5.00 & 13.8 & 25.0 \\
\hline 2-Butanone-d5 & Ave & 0.0228 & 0.0260 & 0.0100 & 57.0 & 50.0 & 13.9 & 40.0 \\
\hline Chloroform-d & Ave & 0.5213 & 0.6021 & 0.0100 & 5.80 & 5.00 & 15.5 & 20.0 \\
\hline 1,2-Dichloroethane-d4 & Ave & 0.1622 & 0.1959 & 0.0100 & 6.00 & 5.00 & 20.7 & 25.0 \\
\hline Benzene-d6 & Ave & 1.454 & 1.546 & 0.0300 & 5.30 & 5.00 & 6.3 & 20.0 \\
\hline 1,2-Dichloropropane-d6 & Ave & 0.3753 & 0.3977 & 0.1000 & 5.30 & 5.00 & 6.0 & 20.0 \\
\hline Toluene-d8 & Ave & 1.411 & 1.571 & 0.2000 & 5.60 & 5.00 & 11.4 & 20.0 \\
\hline trans-1,3-Dichloropropene-d4 & Ave & 0.2267 & 0.2456 & 0.0100 & 5.40 & 5.00 & 8.3 & 25.0 \\
\hline 2-Hexanone-d5 & Ave & 0.0214 & 0.0248 & 0.0100 & 58.0 & 50.0 & 15.7 & 40.0 \\
\hline $1,1,2,2$-Tetrachloroethane-d2 & Ave & 0.1295 & 0.1476 & 0.0100 & 5.70 & 5.00 & 14.0 & 25.0 \\
\hline 1,2-Dichlorobenzene-d4 & Ave & 0.8154 & 0.7984 & 0.0600 & 4.90 & 5.00 & $-2 \cdot 1$ & 20.0 \\
\hline
\end{tabular}


FORM VII

GC/MS VOA CONTINUING CALIBRATION DATA

Lab Name: TestAmerica Burlington

SDG No.: 200-38792-1

Lab Sample ID: CCVC 200-117296/22

Instrument ID: CHD.i

GC Column: DB-624

ID: $0.20(\mathrm{~mm})$

Lab File ID: 25366_22.D

EPA Sample No.: VSTD005EU
Job No.: 200-38792-1

Calibration Date: 06/07/2017 00:03

Calib Start Date: 05/23/2017 17:32

Calib End Date: 05/23/2017 19:16

Conc. Units: ug/L Heated Purge: (Y/N) N

\begin{tabular}{|c|c|c|c|c|c|c|c|c|}
\hline ANALYTE & $\begin{array}{l}\text { CURVE } \\
\text { TYPE }\end{array}$ & AVE RRF & $\mathrm{RRF}$ & MIN RRF & $\begin{array}{c}\text { CALC } \\
\text { AMOUNT }\end{array}$ & $\begin{array}{c}\text { SPIKE } \\
\text { AMOUNT }\end{array}$ & $\circ \mathrm{D}$ & $\begin{array}{l}\text { MAX } \\
\div D\end{array}$ \\
\hline Dichlorodifluoromethane & Ave & 0.3139 & 0.2703 & 0.0100 & 4.30 & 5.00 & -13.9 & 50.0 \\
\hline Chloromethane & Ave & 0.2416 & 0.2382 & 0.0100 & 4.90 & 5.00 & -1.4 & 50.0 \\
\hline Vinyl chloride & Ave & 0.2289 & 0.2353 & 0.0100 & 5.10 & 5.00 & 2.8 & 50.0 \\
\hline Bromomethane & Ave & 0.0965 & 0.0458 & 0.0100 & 2.40 & 5.00 & $-52.5 *$ & 50.0 \\
\hline Chloroethane & Ave & 0.1339 & 0.1603 & 0.0100 & 6.00 & 5.00 & 19.7 & 50.0 \\
\hline Trichlorofluoromethane & Ave & 0.4917 & 0.5472 & 0.0100 & 5.60 & 5.00 & 11.3 & 50.0 \\
\hline $\begin{array}{l}1,1,2 \text {-Trichloro-1,2,2-triflu } \\
\text { oroethane }\end{array}$ & Ave & 0.2474 & 0.2804 & 0.0100 & 5.70 & 5.00 & 13.3 & 50.0 \\
\hline 1,1-Dichloroethene & Ave & 0.2111 & 0.2399 & 0.0200 & 5.70 & 5.00 & 13.6 & 25.0 \\
\hline Acetone & Ave & 0.0108 & 0.0117 & 0.0100 & 54.0 & 50.0 & 7.9 & 50.0 \\
\hline Carbon disulfide & Ave & 0.6624 & 0.6297 & 0.0100 & 4.80 & 5.00 & -4.9 & 25.0 \\
\hline Methyl acetate & Ave & 0.0396 & 0.0301 & 0.0100 & 3.80 & 5.00 & -23.8 & 50.0 \\
\hline Methylene Chloride & Ave & 0.2149 & 0.2149 & 0.0100 & 5.00 & 5.00 & -0.0 & 50.0 \\
\hline Methyl tert-butyl ether & Ave & 0.2639 & 0.2892 & 0.0100 & 5.50 & 5.00 & 9.6 & 50.0 \\
\hline trans-1,2-Dichloroethene & Ave & 0.3101 & 0.3324 & 0.0700 & 5.40 & 5.00 & 7.2 & 25.0 \\
\hline 1,1-Dichloroethane & Ave & 0.5146 & 0.5719 & 0.1000 & 5.60 & 5.00 & 11.1 & 25.0 \\
\hline cis-1,2-Dichloroethene & Ave & 0.2924 & 0.3255 & 0.1000 & 5.60 & 5.00 & 11.3 & 25.0 \\
\hline 2-Butanone & Ave & 0.0223 & 0.0269 & 0.0100 & 60.0 & 50.0 & 20.7 & 50.0 \\
\hline Bromochloromethane & Ave & 0.0968 & 0.1091 & 0.0200 & 5.60 & 5.00 & 12.7 & 25.0 \\
\hline Chloroform & Ave & 0.4942 & 0.5685 & 0.0400 & 5.70 & 5.00 & 15.0 & 25.0 \\
\hline $1,1,1$-Trichloroethane & Ave & 0.7494 & 0.7759 & 0.0500 & 5.20 & 5.00 & 3.5 & 25.0 \\
\hline Cyclohexane & Ave & 0.6809 & 0.7171 & 0.1000 & 5.30 & 5.00 & 5.3 & 50.0 \\
\hline Carbon tetrachloride & Ave & 0.7217 & 0.7562 & 0.0200 & 5.20 & 5.00 & 4.8 & 50.0 \\
\hline Benzene & Ave & 1.490 & 1.618 & 0.3000 & 5.40 & 5.00 & 8.6 & 25.0 \\
\hline 1,2-Dichloroethane & Ave & 0.2120 & 0.2532 & 0.0100 & 6.00 & 5.00 & 19.4 & 50.0 \\
\hline Trichloroethene & Ave & 0.4234 & 0.4250 & 0.1000 & 5.00 & 5.00 & 0.4 & 25.0 \\
\hline Methylcyclohexane & Ave & 0.5779 & 0.6451 & 0.2000 & 5.60 & 5.00 & 11.6 & 50.0 \\
\hline 1,2-Dichloropropane & Ave & 0.2871 & 0.3231 & 0.1000 & 5.60 & 5.00 & 12.5 & 25.0 \\
\hline Bromodichloromethane & Ave & 0.3586 & 0.3892 & 0.0900 & 5.40 & 5.00 & 8.5 & 25.0 \\
\hline cis-1,3-Dichloropropene & Ave & 0.3705 & 0.4086 & 0.1000 & 5.50 & 5.00 & 10.3 & 25.0 \\
\hline 4-Methyl-2-pentanone & Ave & 0.0652 & 0.0790 & 0.0100 & 61.0 & 50.0 & 21.2 & 50.0 \\
\hline Toluene & Ave & 1.636 & 1.904 & 0.4000 & 5.80 & 5.00 & 16.4 & 25.0 \\
\hline trans-1,3-Dichloropropene & Ave & 0.2641 & 0.2837 & 0.0100 & 5.40 & 5.00 & 7.4 & 25.0 \\
\hline 1,1,2-Trichloroethane & Ave & 0.1475 & 0.1664 & 0.0400 & 5.60 & 5.00 & 12.8 & 25.0 \\
\hline Tetrachloroethene & Ave & 0.4449 & 0.4592 & 0.1000 & 5.20 & 5.00 & 3.2 & 25.0 \\
\hline 2-Hexanone & Ave & 0.0446 & 0.0535 & 0.0100 & 60.0 & 50.0 & 20.0 & 50.0 \\
\hline Dibromochloromethane & Ave & 0.2185 & 0.2461 & 0.0500 & 5.60 & 5.00 & 12.6 & 25.0 \\
\hline 1,2-Dibromoethane & Ave & 0.1292 & 0.1389 & 0.0100 & 5.40 & 5.00 & 7.5 & 25.0 \\
\hline Chlorobenzene & Ave & 1.071 & 1.139 & 0.4000 & 5.30 & 5.00 & 6.4 & 25.0 \\
\hline Ethylbenzene & Ave & 1.886 & 2.123 & 0.5000 & 5.60 & 5.00 & 12.6 & 25.0 \\
\hline
\end{tabular}

FORM VII SOM02.2/VOA_Tr 
FORM VII

GC/MS VOA CONTINUING CALIBRATION DATA

Lab Name: TestAmerica Burlington

SDG No.: 200-38792-1

Lab Sample ID: CCVC 200-117296/22

Instrument ID: CHD.i

GC Column: DB-624

Lab File ID: 25366 22.D

EPA Sample No.: VSTD005EU
Job No.: 200-38792-1

Calibration Date: 06/07/2017 00:03

Calib Start Date: 05/23/2017 17:32

Calib End Date: 05/23/2017 19:16

Conc. Units: ug/L Heated Purge: (Y/N) N

\begin{tabular}{|c|c|c|c|c|c|c|c|c|}
\hline ANALYTE & $\begin{array}{c}\text { CURVE } \\
\text { TYPE }\end{array}$ & AVE RRF & RRF & MIN RRF & $\begin{array}{c}\text { CALC } \\
\text { AMOUNT }\end{array}$ & $\begin{array}{c}\text { SPIKE } \\
\text { AMOUNT }\end{array}$ & $\div D$ & $\begin{array}{l}\mathrm{MAX} \\
\div \mathrm{D}\end{array}$ \\
\hline $\mathrm{m}, \mathrm{p}$-Xylene & Ave & 0.7658 & 0.8694 & 0.2000 & 5.70 & 5.00 & 13.5 & 25.0 \\
\hline o-Xylene & Ave & 0.6733 & 0.7808 & 0.3000 & 5.80 & 5.00 & 16.0 & 25.0 \\
\hline Styrene & Ave & 1.011 & 1.216 & 0.2000 & 6.00 & 5.00 & 20.2 & 25.0 \\
\hline Bromoform & Ave & 0.2328 & 0.2233 & 0.0100 & 4.80 & 5.00 & -4.1 & 50.0 \\
\hline Isopropylbenzene & Ave & 2.023 & 2.326 & 0.7000 & 5.70 & 5.00 & 14.9 & 25.0 \\
\hline $1,1,2,2$-Tetrachloroethane & Ave & 0.1281 & 0.1461 & 0.0500 & 5.70 & 5.00 & 14.0 & 25.0 \\
\hline 1,3-Dichlorobenzene & Ave & 1.695 & 1.583 & 0.5000 & 4.70 & 5.00 & -6.6 & 25.0 \\
\hline 1,4-Dichlorobenzene & Ave & 1.776 & 1.696 & 0.7000 & 4.80 & 5.00 & -4.5 & 25.0 \\
\hline 1,2-Dichlorobenzene & Ave & 1.344 & 1.346 & 0.4000 & 5.00 & 5.00 & 0.1 & 25.0 \\
\hline 1,2-Dibromo-3-chloropropane & Ave & 0.0367 & 0.0354 & 0.0100 & 4.80 & 5.00 & -3.5 & 50.0 \\
\hline 1,2,4-Trichlorobenzene & Ave & 0.7703 & 0.6122 & 0.3000 & 4.00 & 5.00 & -20.5 & 50.0 \\
\hline 1,2,3-Trichlorobenzene & Ave & 0.5246 & 0.3951 & 0.2000 & 3.80 & 5.00 & $-24 \cdot 7$ & 50.0 \\
\hline Vinyl chloride-d3 & Ave & 0.1862 & 0.1916 & 0.0100 & 5.10 & 5.00 & 2.9 & 50.0 \\
\hline Chloroethane-d5 & Ave & 0.1386 & 0.1608 & 0.0100 & 5.80 & 5.00 & 16.0 & 50.0 \\
\hline 1,1-Dichloroethene-d2 & Ave & 0.4559 & 0.5128 & 0.0100 & 5.60 & 5.00 & 12.5 & 25.0 \\
\hline 2-Butanone-d5 & Ave & 0.0228 & 0.0253 & 0.0100 & 55.0 & 50.0 & 10.7 & 50.0 \\
\hline Chloroform-d & Ave & 0.5213 & 0.6090 & 0.0100 & 5.80 & 5.00 & 16.8 & 25.0 \\
\hline 1,2-Dichloroethane-d4 & Ave & 0.1622 & 0.1971 & 0.0100 & 6.10 & 5.00 & 21.5 & 25.0 \\
\hline Benzene-d6 & Ave & 1.454 & 1.559 & 0.0300 & 5.40 & 5.00 & 7.2 & 25.0 \\
\hline 1,2-Dichloropropane-d6 & Ave & 0.3753 & 0.4223 & 0.1000 & 5.60 & 5.00 & 12.5 & 25.0 \\
\hline Toluene-d8 & Ave & 1.411 & 1.596 & 0.2000 & 5.70 & 5.00 & 13.1 & 25.0 \\
\hline trans-1,3-Dichloropropene-d4 & Ave & 0.2267 & 0.2324 & 0.0100 & 5.10 & 5.00 & 2.5 & 25.0 \\
\hline 2-Hexanone-d5 & Ave & 0.0214 & 0.0260 & 0.0100 & 61.0 & 50.0 & 21.5 & 50.0 \\
\hline $1,1,2,2$-Tetrachloroethane-d2 & Ave & 0.1295 & 0.1480 & 0.0100 & 5.70 & 5.00 & 14.3 & 25.0 \\
\hline 1,2-Dichlorobenzene-d4 & Ave & 0.8154 & 0.7779 & 0.0600 & 4.80 & 5.00 & -4.6 & 25.0 \\
\hline
\end{tabular}


FORM VII

GC/MS VOA CONTINUING CALIBRATION DATA

Lab Name: TestAmerica Burlington

SDG No.: 200-38792-1

Lab Sample ID: CCVC 200-117296/23

Instrument ID: CHD.i

GC Column: DB-624

ID: $0.20(\mathrm{~mm})$

Lab File ID: 25366_23.D

EPA Sample No.: VSTD005EV
Job No.: 200-38792-1

Calibration Date: 06/07/2017 00:28

Calib Start Date: 05/23/2017 17:32

Calib End Date: 05/23/2017 19:16

Conc. Units: ug/L Heated Purge: (Y/N) N

\begin{tabular}{|c|c|c|c|c|c|c|c|c|}
\hline ANALYTE & $\begin{array}{l}\text { CURVE } \\
\text { TYPE }\end{array}$ & AVE RRF & $\mathrm{RRF}$ & MIN RRF & $\begin{array}{c}\text { CALC } \\
\text { AMOUNT }\end{array}$ & $\begin{array}{c}\text { SPIKE } \\
\text { AMOUNT }\end{array}$ & $\circ \mathrm{D}$ & $\begin{array}{l}\text { MAX } \\
\div D\end{array}$ \\
\hline Dichlorodifluoromethane & Ave & 0.3139 & 0.2697 & 0.0100 & 4.30 & 5.00 & -14.1 & 50.0 \\
\hline Chloromethane & Ave & 0.2416 & 0.2371 & 0.0100 & 4.90 & 5.00 & -1.8 & 50.0 \\
\hline Vinyl chloride & Ave & 0.2289 & 0.2386 & 0.0100 & 5.20 & 5.00 & 4.2 & 50.0 \\
\hline Bromomethane & Ave & 0.0965 & 0.0681 & 0.0100 & 3.50 & 5.00 & -29.5 & 50.0 \\
\hline Chloroethane & Ave & 0.1339 & 0.1583 & 0.0100 & 5.90 & 5.00 & 18.2 & 50.0 \\
\hline Trichlorofluoromethane & Ave & 0.4917 & 0.5399 & 0.0100 & 5.50 & 5.00 & 9.8 & 50.0 \\
\hline $\begin{array}{l}1,1,2 \text {-Trichloro-1,2,2-triflu } \\
\text { oroethane }\end{array}$ & Ave & 0.2474 & 0.2768 & 0.0100 & 5.60 & 5.00 & 11.9 & 50.0 \\
\hline 1,1-Dichloroethene & Ave & 0.2111 & 0.2412 & 0.0200 & 5.70 & 5.00 & 14.2 & 25.0 \\
\hline Acetone & Ave & 0.0108 & 0.0120 & 0.0100 & 55.0 & 50.0 & 10.7 & 50.0 \\
\hline Carbon disulfide & Ave & 0.6624 & 0.6228 & 0.0100 & 4.70 & 5.00 & -6.0 & 25.0 \\
\hline Methyl acetate & Ave & 0.0396 & 0.0336 & 0.0100 & 4.20 & 5.00 & -15.0 & 50.0 \\
\hline Methylene Chloride & Ave & 0.2149 & 0.2417 & 0.0100 & 5.60 & 5.00 & 12.5 & 50.0 \\
\hline Methyl tert-butyl ether & Ave & 0.2639 & 0.2953 & 0.0100 & 5.60 & 5.00 & 11.9 & 50.0 \\
\hline trans-1,2-Dichloroethene & Ave & 0.3101 & 0.3363 & 0.0700 & 5.40 & 5.00 & 8.4 & 25.0 \\
\hline 1,1-Dichloroethane & Ave & 0.5146 & 0.5724 & 0.1000 & 5.60 & 5.00 & 11.2 & 25.0 \\
\hline cis-1,2-Dichloroethene & Ave & 0.2924 & 0.3303 & 0.1000 & 5.60 & 5.00 & 13.0 & 25.0 \\
\hline 2-Butanone & Ave & 0.0223 & 0.0274 & 0.0100 & 61.0 & 50.0 & 22.8 & 50.0 \\
\hline Bromochloromethane & Ave & 0.0968 & 0.1100 & 0.0200 & 5.70 & 5.00 & 13.6 & 25.0 \\
\hline Chloroform & Ave & 0.4942 & 0.5690 & 0.0400 & 5.80 & 5.00 & 15.1 & 25.0 \\
\hline $1,1,1$-Trichloroethane & Ave & 0.7494 & 0.7766 & 0.0500 & 5.20 & 5.00 & 3.6 & 25.0 \\
\hline Cyclohexane & Ave & 0.6809 & 0.7234 & 0.1000 & 5.30 & 5.00 & 6.2 & 50.0 \\
\hline Carbon tetrachloride & Ave & 0.7217 & 0.7462 & 0.0200 & 5.20 & 5.00 & 3.4 & 50.0 \\
\hline Benzene & Ave & 1.490 & 1.627 & 0.3000 & 5.50 & 5.00 & 9.2 & 25.0 \\
\hline 1,2-Dichloroethane & Ave & 0.2120 & 0.2537 & 0.0100 & 6.00 & 5.00 & 19.7 & 50.0 \\
\hline Trichloroethene & Ave & 0.4234 & 0.4245 & 0.1000 & 5.00 & 5.00 & 0.2 & 25.0 \\
\hline Methylcyclohexane & Ave & 0.5779 & 0.6418 & 0.2000 & 5.60 & 5.00 & 11.1 & 50.0 \\
\hline 1,2-Dichloropropane & Ave & 0.2871 & 0.3187 & 0.1000 & 5.50 & 5.00 & 11.0 & 25.0 \\
\hline Bromodichloromethane & Ave & 0.3586 & 0.3955 & 0.0900 & 5.50 & 5.00 & 10.3 & 25.0 \\
\hline cis-1,3-Dichloropropene & Ave & 0.3705 & 0.4093 & 0.1000 & 5.50 & 5.00 & 10.5 & 25.0 \\
\hline 4-Methyl-2-pentanone & Ave & 0.0652 & 0.0782 & 0.0100 & 60.0 & 50.0 & 19.9 & 50.0 \\
\hline Toluene & Ave & 1.636 & 1.887 & 0.4000 & 5.80 & 5.00 & 15.3 & 25.0 \\
\hline trans-1,3-Dichloropropene & Ave & 0.2641 & 0.2866 & 0.0100 & 5.40 & 5.00 & 8.5 & 25.0 \\
\hline 1,1,2-Trichloroethane & Ave & 0.1475 & 0.1679 & 0.0400 & 5.70 & 5.00 & 13.9 & 25.0 \\
\hline Tetrachloroethene & Ave & 0.4449 & 0.4584 & 0.1000 & 5.10 & 5.00 & 3.0 & 25.0 \\
\hline 2-Hexanone & Ave & 0.0446 & 0.0545 & 0.0100 & 61.0 & 50.0 & 22.3 & 50.0 \\
\hline Dibromochloromethane & Ave & 0.2185 & 0.2449 & 0.0500 & 5.60 & 5.00 & 12.1 & 25.0 \\
\hline 1,2-Dibromoethane & Ave & 0.1292 & 0.1410 & 0.0100 & 5.50 & 5.00 & 9.2 & 25.0 \\
\hline Chlorobenzene & Ave & 1.071 & 1.143 & 0.4000 & 5.30 & 5.00 & 6.8 & 25.0 \\
\hline Ethylbenzene & Ave & 1.886 & 2.137 & 0.5000 & 5.70 & 5.00 & 13.3 & 25.0 \\
\hline
\end{tabular}

FORM VII SOM02.2/VOA_Tr 
FORM VII

GC/MS VOA CONTINUING CALIBRATION DATA

Lab Name: TestAmerica Burlington

SDG No.: 200-38792-1

Lab Sample ID: CCVC 200-117296/23

Instrument ID: CHD.i

GC Column: DB-624

ID $: 0.20(\mathrm{~mm})$

Lab File ID: 25366 23.D

EPA Sample No.: VSTD005EV
Job No.: 200-38792-1

Calibration Date: 06/07/2017 00:28

Calib Start Date: 05/23/2017 17:32

Calib End Date: 05/23/2017 19:16

Conc. Units: ug/L Heated Purge: (Y/N) N

\begin{tabular}{|c|c|c|c|c|c|c|c|c|}
\hline ANALYTE & $\begin{array}{c}\text { CURVE } \\
\text { TYPE }\end{array}$ & AVE RRF & RRF & MIN RRF & $\begin{array}{c}\text { CALC } \\
\text { AMOUNT }\end{array}$ & $\begin{array}{c}\text { SPIKE } \\
\text { AMOUNT }\end{array}$ & $\div D$ & $\begin{array}{l}\mathrm{MAX} \\
\div \mathrm{D}\end{array}$ \\
\hline $\mathrm{m}, \mathrm{p}$-Xylene & Ave & 0.7658 & 0.8696 & 0.2000 & 5.70 & 5.00 & 13.6 & 25.0 \\
\hline o-Xylene & Ave & 0.6733 & 0.7859 & 0.3000 & 5.80 & 5.00 & 16.7 & 25.0 \\
\hline Styrene & Ave & 1.011 & 1.225 & 0.2000 & 6.10 & 5.00 & 21.2 & 25.0 \\
\hline Bromoform & Ave & 0.2328 & 0.2319 & 0.0100 & 5.00 & 5.00 & -0.4 & 50.0 \\
\hline Isopropylbenzene & Ave & 2.023 & 2.340 & 0.7000 & 5.80 & 5.00 & 15.6 & 25.0 \\
\hline $1,1,2,2$-Tetrachloroethane & Ave & 0.1281 & 0.1489 & 0.0500 & 5.80 & 5.00 & 16.2 & 25.0 \\
\hline 1,3-Dichlorobenzene & Ave & 1.695 & 1.635 & 0.5000 & 4.80 & 5.00 & -3.5 & 25.0 \\
\hline 1,4-Dichlorobenzene & Ave & 1.776 & 1.739 & 0.7000 & 4.90 & 5.00 & -2.0 & 25.0 \\
\hline 1,2-Dichlorobenzene & Ave & 1.344 & 1.384 & 0.4000 & 5.10 & 5.00 & 3.0 & 25.0 \\
\hline 1,2-Dibromo-3-chloropropane & Ave & 0.0367 & 0.0371 & 0.0100 & 5.00 & 5.00 & 1.0 & 50.0 \\
\hline 1,2,4-Trichlorobenzene & Ave & 0.7703 & 0.6503 & 0.3000 & 4.20 & 5.00 & $-15 \cdot 6$ & 50.0 \\
\hline 1,2,3-Trichlorobenzene & Ave & 0.5246 & 0.4341 & 0.2000 & 4.10 & 5.00 & -17.3 & 50.0 \\
\hline Vinyl chloride-d3 & Ave & 0.1862 & 0.1930 & 0.0100 & 5.20 & 5.00 & 3.7 & 50.0 \\
\hline Chloroethane-d5 & Ave & 0.1386 & 0.1597 & 0.0100 & 5.80 & 5.00 & 15.3 & 50.0 \\
\hline 1,1-Dichloroethene-d2 & Ave & 0.4559 & 0.5141 & 0.0100 & 5.60 & 5.00 & 12.8 & 25.0 \\
\hline 2-Butanone-d5 & Ave & 0.0228 & 0.0261 & 0.0100 & 57.0 & 50.0 & 14.4 & 50.0 \\
\hline Chloroform-d & Ave & 0.5213 & 0.6059 & 0.0100 & 5.80 & 5.00 & 16.2 & 25.0 \\
\hline 1,2-Dichloroethane-d4 & Ave & 0.1622 & 0.1963 & 0.0100 & 6.00 & 5.00 & 21.0 & 25.0 \\
\hline Benzene-d6 & Ave & 1.454 & 1.549 & 0.0300 & 5.30 & 5.00 & 6.5 & 25.0 \\
\hline 1,2-Dichloropropane-d6 & Ave & 0.3753 & 0.4149 & 0.1000 & 5.50 & 5.00 & 10.6 & 25.0 \\
\hline Toluene-d8 & Ave & 1.411 & 1.591 & 0.2000 & 5.60 & 5.00 & 12.7 & 25.0 \\
\hline trans-1,3-Dichloropropene-d4 & Ave & 0.2267 & 0.2343 & 0.0100 & 5.20 & 5.00 & 3.3 & 25.0 \\
\hline 2-Hexanone-d5 & Ave & 0.0214 & 0.0258 & 0.0100 & 60.0 & 50.0 & 20.6 & 50.0 \\
\hline $1,1,2,2$-Tetrachloroethane-d2 & Ave & 0.1295 & 0.1468 & 0.0100 & 5.70 & 5.00 & 13.3 & 25.0 \\
\hline 1,2-Dichlorobenzene-d4 & Ave & 0.8154 & 0.7896 & 0.0600 & 4.80 & 5.00 & -3.2 & 25.0 \\
\hline
\end{tabular}


FORM VII

GC/MS VOA CONTINUING CALIBRATION DATA

Lab Name: TestAmerica Burlington

SDG No.: 200-38792-1

Lab Sample ID: CCVIS 200-117409/5

Instrument ID: CHD.i

GC Column: DB-624

ID: $0.20(\mathrm{~mm})$

Lab File ID: 25405 05.D

EPA Sample No.: VSTD005EW
Job No.: 200-38792-1

Calibration Date: 06/08/2017 15:53

Calib Start Date: 05/23/2017 17:32

Calib End Date: 05/23/2017 19:16

Conc. Units: ug/L Heated Purge: (Y/N) N

\begin{tabular}{|c|c|c|c|c|c|c|c|c|}
\hline ANALYTE & $\begin{array}{c}\text { CURVE } \\
\text { TYPE }\end{array}$ & AVE $\quad R R F$ & $\mathrm{RRF}$ & MIN RRF & $\begin{array}{c}\text { CALC } \\
\text { AMOUNT }\end{array}$ & $\begin{array}{c}\text { SPIKE } \\
\text { AMOUNT }\end{array}$ & $\div D$ & $\begin{array}{l}\text { MAX } \\
\div D\end{array}$ \\
\hline Dichlorodifluoromethane & Ave & 0.3139 & 0.2440 & 0.0100 & 3.90 & 5.00 & -22.3 & 40.0 \\
\hline Chloromethane & Ave & 0.2416 & 0.1931 & 0.0100 & 4.00 & 5.00 & -20.1 & 30.0 \\
\hline Vinyl chloride & Ave & 0.2289 & 0.2202 & 0.0100 & 4.80 & 5.00 & -3.8 & 30.0 \\
\hline Bromomethane & Ave & 0.0965 & 0.0732 & 0.0100 & 3.80 & 5.00 & -24.2 & 30.0 \\
\hline Chloroethane & Ave & 0.1339 & 0.1386 & 0.0100 & 5.20 & 5.00 & 3.5 & 30.0 \\
\hline Trichlorofluoromethane & Ave & 0.4917 & 0.4082 & 0.0100 & 4.10 & 5.00 & -17.0 & 30.0 \\
\hline $\begin{array}{l}1,1,2 \text {-Trichloro-1,2,2-triflu } \\
\text { oroethane }\end{array}$ & Ave & 0.2474 & 0.2263 & 0.0100 & 4.60 & 5.00 & -8.5 & 30.0 \\
\hline 1,1-Dichloroethene & Ave & 0.2111 & 0.2081 & 0.0200 & 4.90 & 5.00 & -1.4 & 20.0 \\
\hline Acetone & Ave & 0.0108 & $0.0085 *$ & 0.0100 & 39.0 & 50.0 & -21.8 & 40.0 \\
\hline Carbon disulfide & Ave & 0.6624 & 0.5251 & 0.0100 & 4.00 & 5.00 & -20.7 & 25.0 \\
\hline Methyl acetate & Ave & 0.0396 & 0.0375 & 0.0100 & 4.70 & 5.00 & -5.1 & 40.0 \\
\hline Methylene Chloride & Ave & 0.2149 & 0.2180 & 0.0100 & 5.10 & 5.00 & 1.4 & 30.0 \\
\hline Methyl tert-butyl ether & Ave & 0.2639 & 0.3200 & 0.0100 & 6.10 & 5.00 & 21.3 & 30.0 \\
\hline trans-1,2-Dichloroethene & Ave & 0.3101 & 0.3146 & 0.0700 & 5.10 & 5.00 & 1.5 & 20.0 \\
\hline 1,1-Dichloroethane & Ave & 0.5146 & 0.4938 & 0.1000 & 4.80 & 5.00 & -4.0 & 20.0 \\
\hline cis-1,2-Dichloroethene & Ave & 0.2924 & 0.3163 & 0.1000 & 5.40 & 5.00 & 8.2 & 20.0 \\
\hline 2-Butanone & Ave & 0.0223 & 0.0224 & 0.0100 & 50.0 & 50.0 & 0.3 & 40.0 \\
\hline Bromochloromethane & Ave & 0.0968 & 0.1018 & 0.0200 & 5.30 & 5.00 & 5.2 & 20.0 \\
\hline Chloroform & Ave & 0.4942 & 0.4698 & 0.0400 & 4.80 & 5.00 & -4.9 & 20.0 \\
\hline $1,1,1$-Trichloroethane & Ave & 0.7494 & 0.6542 & 0.0500 & 4.40 & 5.00 & -12.7 & 20.0 \\
\hline Cyclohexane & Ave & 0.6809 & 0.6754 & 0.1000 & 5.00 & 5.00 & -0.8 & 25.0 \\
\hline Carbon tetrachloride & Ave & 0.7217 & 0.6029 & 0.0200 & 4.20 & 5.00 & -16.5 & 25.0 \\
\hline Benzene & Ave & 1.490 & 1.450 & 0.3000 & 4.90 & 5.00 & -2.7 & 20.0 \\
\hline 1,2-Dichloroethane & Ave & 0.2120 & 0.2001 & 0.0100 & 4.70 & 5.00 & -5.6 & 25.0 \\
\hline Trichloroethene & Ave & 0.4234 & 0.4090 & 0.1000 & 4.80 & 5.00 & -3.4 & 20.0 \\
\hline Methylcyclohexane & Ave & 0.5779 & 0.5395 & 0.2000 & 4.70 & 5.00 & -6.7 & 25.0 \\
\hline 1,2-Dichloropropane & Ave & 0.2871 & 0.2820 & 0.1000 & 4.90 & 5.00 & -1.8 & 20.0 \\
\hline Bromodichloromethane & Ave & 0.3586 & 0.3351 & 0.0900 & 4.70 & 5.00 & -6.6 & 20.0 \\
\hline cis-1,3-Dichloropropene & Ave & 0.3705 & 0.3993 & 0.1000 & 5.40 & 5.00 & 7.8 & 20.0 \\
\hline 4-Methyl-2-pentanone & Ave & 0.0652 & 0.0671 & 0.0100 & 51.0 & 50.0 & 3.0 & 30.0 \\
\hline Toluene & Ave & 1.636 & 1.662 & 0.4000 & 5.10 & 5.00 & 1.6 & 20.0 \\
\hline trans-1,3-Dichloropropene & Ave & 0.2641 & 0.2760 & 0.0100 & 5.20 & 5.00 & 4.5 & 20.0 \\
\hline 1,1,2-Trichloroethane & Ave & 0.1475 & 0.1478 & 0.0400 & 5.00 & 5.00 & 0.2 & 20.0 \\
\hline Tetrachloroethene & Ave & 0.4449 & 0.4332 & 0.1000 & 4.90 & 5.00 & -2.6 & 20.0 \\
\hline 2-Hexanone & Ave & 0.0446 & 0.0449 & 0.0100 & 50.0 & 50.0 & 0.8 & 40.0 \\
\hline Dibromochloromethane & Ave & 0.2185 & 0.2133 & 0.0500 & 4.90 & 5.00 & -2.4 & 20.0 \\
\hline 1,2-Dibromoethane & Ave & 0.1292 & 0.1385 & 0.0100 & 5.40 & 5.00 & 7.2 & 20.0 \\
\hline Chlorobenzene & Ave & 1.071 & 1.048 & 0.4000 & 4.90 & 5.00 & -2.1 & 20.0 \\
\hline Ethylbenzene & Ave & 1.886 & 1.930 & 0.5000 & 5.10 & 5.00 & 2.4 & 20.0 \\
\hline
\end{tabular}

FORM VII SOM02.2/VOA_Tr 
FORM VII

GC/MS VOA CONTINUING CALIBRATION DATA

Lab Name: TestAmerica Burlington

SDG No.: 200-38792-1

Lab Sample ID: CCVIS 200-117409/5

Instrument ID: CHD.i

GC Column: DB-624

Lab File ID: 25405_05.D

EPA Sample No.: VSTD005EW
Job No.: 200-38792-1

Calibration Date: 06/08/2017 15:53

Calib Start Date: 05/23/2017 17:32

Calib End Date: 05/23/2017 19:16

Conc. Units: ug/L Heated Purge: (Y/N) N

\begin{tabular}{|c|c|c|c|c|c|c|c|c|}
\hline ANALYTE & $\begin{array}{c}\text { CURVE } \\
\text { TYPE }\end{array}$ & AVE RRF & RRF & MIN RRF & $\begin{array}{c}\text { CALC } \\
\text { AMOUNT }\end{array}$ & $\begin{array}{c}\text { SPIKE } \\
\text { AMOUNT }\end{array}$ & $\div D$ & $\begin{array}{l}\mathrm{MAX} \\
\div \mathrm{D}\end{array}$ \\
\hline $\mathrm{m}, \mathrm{p}$-Xylene & Ave & 0.7658 & 0.8001 & 0.2000 & 5.20 & 5.00 & 4.5 & 20.0 \\
\hline o-Xylene & Ave & 0.6733 & 0.7222 & 0.3000 & 5.40 & 5.00 & 7.3 & 20.0 \\
\hline Styrene & Ave & 1.011 & 1.067 & 0.2000 & 5.30 & 5.00 & 5.5 & 20.0 \\
\hline Bromoform & Ave & 0.2328 & 0.2179 & 0.0100 & 4.70 & 5.00 & -6.4 & 30.0 \\
\hline Isopropylbenzene & Ave & 2.023 & 2.083 & 0.7000 & 5.10 & 5.00 & 2.9 & 25.0 \\
\hline $1,1,2,2$-Tetrachloroethane & Ave & 0.1281 & 0.1279 & 0.0500 & 5.00 & 5.00 & -0.1 & 25.0 \\
\hline 1,3-Dichlorobenzene & Ave & 1.695 & 1.733 & 0.5000 & 5.10 & 5.00 & 2.2 & 20.0 \\
\hline 1,4-Dichlorobenzene & Ave & 1.776 & 1.649 & 0.7000 & 4.60 & 5.00 & -7.1 & 20.0 \\
\hline 1,2-Dichlorobenzene & Ave & 1.344 & 1.345 & 0.4000 & 5.00 & 5.00 & 0.0 & 20.0 \\
\hline 1,2-Dibromo-3-chloropropane & Ave & 0.0367 & 0.0331 & 0.0100 & 4.50 & 5.00 & -10.0 & 40.0 \\
\hline 1,2,4-Trichlorobenzene & Ave & 0.7703 & 0.7895 & 0.3000 & 5.10 & 5.00 & 2.5 & 30.0 \\
\hline 1,2,3-Trichlorobenzene & Ave & 0.5246 & 0.5420 & 0.2000 & 5.20 & 5.00 & 3.3 & 40.0 \\
\hline Vinyl chloride-d3 & Ave & 0.1862 & 0.1819 & 0.0100 & 4.90 & 5.00 & -2.3 & 30.0 \\
\hline Chloroethane-d5 & Ave & 0.1386 & 0.1335 & 0.0100 & 4.80 & 5.00 & -3.7 & 30.0 \\
\hline 1,1-Dichloroethene-d2 & Ave & 0.4559 & 0.4147 & 0.0100 & 4.50 & 5.00 & -9.0 & 25.0 \\
\hline 2-Butanone-d5 & Ave & 0.0228 & 0.0236 & 0.0100 & 52.0 & 50.0 & 3.4 & 40.0 \\
\hline Chloroform-d & Ave & 0.5213 & 0.4998 & 0.0100 & 4.80 & 5.00 & -4.1 & 20.0 \\
\hline 1,2-Dichloroethane-d4 & Ave & 0.1622 & 0.1515 & 0.0100 & 4.70 & 5.00 & -6.7 & 25.0 \\
\hline Benzene-d6 & Ave & 1.454 & 1.449 & 0.0300 & 5.00 & 5.00 & -0.4 & 20.0 \\
\hline 1,2-Dichloropropane-d6 & Ave & 0.3753 & 0.3568 & 0.1000 & 4.80 & 5.00 & -4.9 & 20.0 \\
\hline Toluene-d8 & Ave & 1.411 & 1.442 & 0.2000 & 5.10 & 5.00 & 2.2 & 20.0 \\
\hline trans-1,3-Dichloropropene-d4 & Ave & 0.2267 & 0.2374 & 0.0100 & 5.20 & 5.00 & 4.7 & 25.0 \\
\hline 2-Hexanone-d5 & Ave & 0.0214 & 0.0266 & 0.0100 & 62.0 & 50.0 & 24.2 & 40.0 \\
\hline $1,1,2,2$-Tetrachloroethane-d2 & Ave & 0.1295 & 0.1267 & 0.0100 & 4.90 & 5.00 & -2.2 & 25.0 \\
\hline 1,2-Dichlorobenzene-d4 & Ave & 0.8154 & 0.8206 & 0.0600 & 5.00 & 5.00 & 0.6 & 20.0 \\
\hline
\end{tabular}


FORM VII

GC/MS VOA CONTINUING CALIBRATION DATA

Lab Name: TestAmerica Burlington

SDG No.: 200-38792-1

Lab Sample ID: CCVC 200-117409/22

Instrument ID: CHD.i

GC Column: DB-624

ID: $0.20(\mathrm{~mm})$

Lab File ID: 25405_22.D

EPA Sample No.: VSTD005EX
Job No.: 200-38792-1

Calibration Date: 06/08/2017 23:16

Calib Start Date: 05/23/2017 17:32

Calib End Date: 05/23/2017 19:16

Conc. Units: ug/L Heated Purge: (Y/N) N

\begin{tabular}{|c|c|c|c|c|c|c|c|c|}
\hline ANALYTE & $\begin{array}{l}\text { CURVE } \\
\text { TYPE }\end{array}$ & AVE RRF & $\mathrm{RRF}$ & MIN RRF & $\begin{array}{c}\text { CALC } \\
\text { AMOUNT }\end{array}$ & $\begin{array}{c}\text { SPIKE } \\
\text { AMOUNT }\end{array}$ & $\circ \mathrm{D}$ & $\begin{array}{l}\text { MAX } \\
\div D\end{array}$ \\
\hline Dichlorodifluoromethane & Ave & 0.3139 & 0.2845 & 0.0100 & 4.50 & 5.00 & -9.4 & 50.0 \\
\hline Chloromethane & Ave & 0.2416 & 0.1570 & 0.0100 & 3.20 & 5.00 & -35.0 & 50.0 \\
\hline Vinyl chloride & Ave & 0.2289 & 0.1787 & 0.0100 & 3.90 & 5.00 & -21.9 & 50.0 \\
\hline Bromomethane & Ave & 0.0965 & 0.0125 & 0.0100 & 0.650 & 5.00 & $-87.1 *$ & 50.0 \\
\hline Chloroethane & Ave & 0.1339 & 0.1422 & 0.0100 & 5.30 & 5.00 & 6.2 & 50.0 \\
\hline Trichlorofluoromethane & Ave & 0.4917 & 0.5487 & 0.0100 & 5.60 & 5.00 & 11.6 & 50.0 \\
\hline $\begin{array}{l}1,1,2 \text {-Trichloro-1,2,2-triflu } \\
\text { oroethane }\end{array}$ & Ave & 0.2474 & 0.2601 & 0.0100 & 5.30 & 5.00 & 5.1 & 50.0 \\
\hline 1,1-Dichloroethene & Ave & 0.2111 & 0.2234 & 0.0200 & 5.30 & 5.00 & 5.8 & 25.0 \\
\hline Acetone & Ave & 0.0108 & 0.0117 & 0.0100 & 54.0 & 50.0 & 7.8 & 50.0 \\
\hline Carbon disulfide & Ave & 0.6624 & 0.5752 & 0.0100 & 4.30 & 5.00 & -13.2 & 25.0 \\
\hline Methyl acetate & Ave & 0.0396 & 0.0405 & 0.0100 & 5.10 & 5.00 & 2.4 & 50.0 \\
\hline Methylene Chloride & Ave & 0.2149 & 0.2339 & 0.0100 & 5.40 & 5.00 & 8.8 & 50.0 \\
\hline Methyl tert-butyl ether & Ave & 0.2639 & 0.3086 & 0.0100 & 5.80 & 5.00 & 16.9 & 50.0 \\
\hline trans-1,2-Dichloroethene & Ave & 0.3101 & 0.3209 & 0.0700 & 5.20 & 5.00 & 3.5 & 25.0 \\
\hline 1,1-Dichloroethane & Ave & 0.5146 & 0.5493 & 0.1000 & 5.30 & 5.00 & 6.7 & 25.0 \\
\hline cis-1,2-Dichloroethene & Ave & 0.2924 & 0.3257 & 0.1000 & 5.60 & 5.00 & 11.4 & 25.0 \\
\hline 2-Butanone & Ave & 0.0223 & 0.0249 & 0.0100 & 56.0 & 50.0 & 11.5 & 50.0 \\
\hline Bromochloromethane & Ave & 0.0968 & 0.1098 & 0.0200 & 5.70 & 5.00 & 13.4 & 25.0 \\
\hline Chloroform & Ave & 0.4942 & 0.5666 & 0.0400 & 5.70 & 5.00 & 14.7 & 25.0 \\
\hline $1,1,1$-Trichloroethane & Ave & 0.7494 & 0.7639 & 0.0500 & 5.10 & 5.00 & 1.9 & 25.0 \\
\hline Cyclohexane & Ave & 0.6809 & 0.6372 & 0.1000 & 4.70 & 5.00 & -6.4 & 50.0 \\
\hline Carbon tetrachloride & Ave & 0.7217 & 0.7224 & 0.0200 & 5.00 & 5.00 & 0.0 & 50.0 \\
\hline Benzene & Ave & 1.490 & 1.505 & 0.3000 & 5.10 & 5.00 & 1.1 & 25.0 \\
\hline 1,2-Dichloroethane & Ave & 0.2120 & 0.2626 & 0.0100 & 6.20 & 5.00 & 23.9 & 50.0 \\
\hline Trichloroethene & Ave & 0.4234 & 0.4044 & 0.1000 & 4.80 & 5.00 & -4.5 & 25.0 \\
\hline Methylcyclohexane & Ave & 0.5779 & 0.5565 & 0.2000 & 4.80 & 5.00 & -3.7 & 50.0 \\
\hline 1,2-Dichloropropane & Ave & 0.2871 & 0.2939 & 0.1000 & 5.10 & 5.00 & 2.4 & 25.0 \\
\hline Bromodichloromethane & Ave & 0.3586 & 0.3857 & 0.0900 & 5.40 & 5.00 & 7.6 & 25.0 \\
\hline cis-1,3-Dichloropropene & Ave & 0.3705 & 0.3872 & 0.1000 & 5.20 & 5.00 & 4.5 & 25.0 \\
\hline 4-Methyl-2-pentanone & Ave & 0.0652 & 0.0747 & 0.0100 & 57.0 & 50.0 & 14.6 & 50.0 \\
\hline Toluene & Ave & 1.636 & 1.766 & 0.4000 & 5.40 & 5.00 & 8.0 & 25.0 \\
\hline trans-1,3-Dichloropropene & Ave & 0.2641 & 0.2768 & 0.0100 & 5.20 & 5.00 & 4.8 & 25.0 \\
\hline 1,1,2-Trichloroethane & Ave & 0.1475 & 0.1606 & 0.0400 & 5.40 & 5.00 & 8.9 & 25.0 \\
\hline Tetrachloroethene & Ave & 0.4449 & 0.4281 & 0.1000 & 4.80 & 5.00 & -3.8 & 25.0 \\
\hline 2-Hexanone & Ave & 0.0446 & 0.0520 & 0.0100 & 58.0 & 50.0 & 16.7 & 50.0 \\
\hline Dibromochloromethane & Ave & 0.2185 & 0.2392 & 0.0500 & 5.50 & 5.00 & 9.4 & 25.0 \\
\hline 1,2-Dibromoethane & Ave & 0.1292 & 0.1380 & 0.0100 & 5.30 & 5.00 & 6.9 & 25.0 \\
\hline Chlorobenzene & Ave & 1.071 & 1.108 & 0.4000 & 5.20 & 5.00 & 3.5 & 25.0 \\
\hline Ethylbenzene & Ave & 1.886 & 2.014 & 0.5000 & 5.30 & 5.00 & 6.8 & 25.0 \\
\hline
\end{tabular}

FORM VII SOM02.2/VOA_Tr 
FORM VII

GC/MS VOA CONTINUING CALIBRATION DATA

Lab Name: TestAmerica Burlington

SDG No.: 200-38792-1

Lab Sample ID: CCVC 200-117409/22

Instrument ID: CHD.i

GC Column: DB-624

ID $: 0.20(\mathrm{~mm})$

Lab File ID: 25405 22.D

EPA Sample No.: VSTD005EX
Job No.: 200-38792-1

Calibration Date: 06/08/2017 23:16

Calib Start Date: 05/23/2017 17:32

Calib End Date: 05/23/2017 19:16

Conc. Units: ug/L Heated Purge: (Y/N) N

\begin{tabular}{|c|c|c|c|c|c|c|c|c|}
\hline ANALYTE & $\begin{array}{c}\text { CURVE } \\
\text { TYPE }\end{array}$ & AVE RRF & $\mathrm{RRF}$ & MIN RRF & $\begin{array}{c}\text { CALC } \\
\text { AMOUNT }\end{array}$ & $\begin{array}{c}\text { SPIKE } \\
\text { AMOUNT }\end{array}$ & $\div D$ & $\begin{array}{l}\text { MAX } \\
\because D\end{array}$ \\
\hline $\mathrm{m}, \mathrm{p}$-Xylene & Ave & 0.7658 & 0.8327 & 0.2000 & 5.40 & 5.00 & 8.7 & 25.0 \\
\hline o-Xylene & Ave & 0.6733 & 0.7523 & 0.3000 & 5.60 & 5.00 & 11.7 & 25.0 \\
\hline Styrene & Ave & 1.011 & 1.177 & 0.2000 & 5.80 & 5.00 & 16.4 & 25.0 \\
\hline Bromoform & Ave & 0.2328 & 0.2288 & 0.0100 & 4.90 & 5.00 & -1.7 & 50.0 \\
\hline Isopropylbenzene & Ave & 2.023 & 2.220 & 0.7000 & 5.50 & 5.00 & 9.7 & 25.0 \\
\hline $1,1,2,2$-Tetrachloroethane & Ave & 0.1281 & 0.1431 & 0.0500 & 5.60 & 5.00 & 11.7 & 25.0 \\
\hline 1,3-Dichlorobenzene & Ave & 1.695 & 1.560 & 0.5000 & 4.60 & 5.00 & -8.0 & 25.0 \\
\hline 1,4-Dichlorobenzene & Ave & 1.776 & 1.663 & 0.7000 & 4.70 & 5.00 & -6.4 & 25.0 \\
\hline 1,2-Dichlorobenzene & Ave & 1.344 & 1.348 & 0.4000 & 5.00 & 5.00 & 0.3 & 25.0 \\
\hline 1,2-Dibromo-3-chloropropane & Ave & 0.0367 & 0.0344 & 0.0100 & 4.70 & 5.00 & -6.4 & 50.0 \\
\hline 1,2,4-Trichlorobenzene & Ave & 0.7703 & 0.6477 & 0.3000 & 4.20 & 5.00 & -15.9 & 50.0 \\
\hline 1,2,3-Trichlorobenzene & Ave & 0.5246 & 0.4375 & 0.2000 & 4.20 & 5.00 & -16.6 & 50.0 \\
\hline Vinyl chloride-d3 & Ave & 0.1862 & 0.1451 & 0.0100 & 3.90 & 5.00 & -22.1 & 50.0 \\
\hline Chloroethane-d5 & Ave & 0.1386 & 0.1438 & 0.0100 & 5.20 & 5.00 & 3.8 & 50.0 \\
\hline 1,1-Dichloroethene-d2 & Ave & 0.4559 & 0.4845 & 0.0100 & 5.30 & 5.00 & 6.3 & 25.0 \\
\hline 2-Butanone-d5 & Ave & 0.0228 & 0.0254 & 0.0100 & 56.0 & 50.0 & 11.1 & 50.0 \\
\hline Chloroform-d & Ave & 0.5213 & 0.6011 & 0.0100 & 5.80 & 5.00 & 15.3 & 25.0 \\
\hline 1,2-Dichloroethane-d4 & Ave & 0.1622 & 0.2002 & 0.0100 & 6.20 & 5.00 & 23.4 & 25.0 \\
\hline Benzene-d6 & Ave & 1.454 & 1.472 & 0.0300 & 5.10 & 5.00 & 1.2 & 25.0 \\
\hline 1,2-Dichloropropane-d6 & Ave & 0.3753 & 0.3851 & 0.1000 & 5.10 & 5.00 & 2.6 & 25.0 \\
\hline Toluene-d8 & Ave & 1.411 & 1.520 & 0.2000 & 5.40 & 5.00 & 7.7 & 25.0 \\
\hline trans-1,3-Dichloropropene-d4 & Ave & 0.2267 & 0.2316 & 0.0100 & 5.10 & 5.00 & 2.2 & 25.0 \\
\hline 2-Hexanone-d5 & Ave & 0.0214 & 0.0254 & 0.0100 & 59.0 & 50.0 & 18.4 & 50.0 \\
\hline $1,1,2,2$-Tetrachloroethane-d2 & Ave & 0.1295 & 0.1434 & 0.0100 & 5.50 & 5.00 & 10.7 & 25.0 \\
\hline 1,2-Dichlorobenzene-d4 & Ave & 0.8154 & 0.7765 & 0.0600 & 4.80 & 5.00 & -4.8 & 25.0 \\
\hline
\end{tabular}


FORM VII

GC/MS VOA CONTINUING CALIBRATION DATA

Lab Name: TestAmerica Burlington

SDG No.: 200-38792-1

Lab Sample ID: CCVC 200-117409/23

Instrument ID: CHD.i

GC Column: DB-624

ID: $0.20(\mathrm{~mm})$

Lab File ID: 25405_23.D

EPA Sample No.: VSTD005EY
Job No.: 200-38792-1

Calibration Date: 06/08/2017 23:42

Calib Start Date: 05/23/2017 17:32

Calib End Date: 05/23/2017 19:16

Conc. Units: ug/L Heated Purge: (Y/N) N

\begin{tabular}{|c|c|c|c|c|c|c|c|c|}
\hline ANALYTE & $\begin{array}{l}\text { CURVE } \\
\text { TYPE }\end{array}$ & AVE RRF & $\mathrm{RRF}$ & MIN RRF & $\begin{array}{c}\text { CALC } \\
\text { AMOUNT }\end{array}$ & $\begin{array}{c}\text { SPIKE } \\
\text { AMOUNT }\end{array}$ & $\circ \mathrm{D}$ & $\begin{array}{l}\text { MAX } \\
\div D\end{array}$ \\
\hline Dichlorodifluoromethane & Ave & 0.3139 & 0.2700 & 0.0100 & 4.30 & 5.00 & -14.0 & 50.0 \\
\hline Chloromethane & Ave & 0.2416 & 0.1893 & 0.0100 & 3.90 & 5.00 & -21.6 & 50.0 \\
\hline Vinyl chloride & Ave & 0.2289 & 0.2020 & 0.0100 & 4.40 & 5.00 & -11.7 & 50.0 \\
\hline Bromomethane & Ave & 0.0965 & 0.0113 & 0.0100 & 0.580 & 5.00 & $-88.3 *$ & 50.0 \\
\hline Chloroethane & Ave & 0.1339 & 0.1448 & 0.0100 & 5.40 & 5.00 & 8.1 & 50.0 \\
\hline Trichlorofluoromethane & Ave & 0.4917 & 0.5248 & 0.0100 & 5.30 & 5.00 & 6.7 & 50.0 \\
\hline $\begin{array}{l}1,1,2 \text {-Trichloro-1,2,2-triflu } \\
\text { oroethane }\end{array}$ & Ave & 0.2474 & 0.2496 & 0.0100 & 5.00 & 5.00 & 0.9 & 50.0 \\
\hline 1,1-Dichloroethene & Ave & 0.2111 & 0.2205 & 0.0200 & 5.20 & 5.00 & 4.5 & 25.0 \\
\hline Acetone & Ave & 0.0108 & 0.0116 & 0.0100 & 53.0 & 50.0 & 6.9 & 50.0 \\
\hline Carbon disulfide & Ave & 0.6624 & 0.5703 & 0.0100 & 4.30 & 5.00 & -13.9 & 25.0 \\
\hline Methyl acetate & Ave & 0.0396 & 0.0399 & 0.0100 & 5.00 & 5.00 & 0.8 & 50.0 \\
\hline Methylene Chloride & Ave & 0.2149 & 0.2386 & 0.0100 & 5.50 & 5.00 & 11.0 & 50.0 \\
\hline Methyl tert-butyl ether & Ave & 0.2639 & 0.3108 & 0.0100 & 5.90 & 5.00 & 17.8 & 50.0 \\
\hline trans-1,2-Dichloroethene & Ave & 0.3101 & 0.3176 & 0.0700 & 5.10 & 5.00 & 2.4 & 25.0 \\
\hline 1,1-Dichloroethane & Ave & 0.5146 & 0.5395 & 0.1000 & 5.20 & 5.00 & 4.8 & 25.0 \\
\hline cis-1,2-Dichloroethene & Ave & 0.2924 & 0.3247 & 0.1000 & 5.60 & 5.00 & 11.1 & 25.0 \\
\hline 2-Butanone & Ave & 0.0223 & 0.0257 & 0.0100 & 58.0 & 50.0 & 15.2 & 50.0 \\
\hline Bromochloromethane & Ave & 0.0968 & 0.1115 & 0.0200 & 5.80 & 5.00 & 15.1 & 25.0 \\
\hline Chloroform & Ave & 0.4942 & 0.5570 & 0.0400 & 5.60 & 5.00 & 12.7 & 25.0 \\
\hline $1,1,1$-Trichloroethane & Ave & 0.7494 & 0.7543 & 0.0500 & 5.00 & 5.00 & 0.6 & 25.0 \\
\hline Cyclohexane & Ave & 0.6809 & 0.6234 & 0.1000 & 4.60 & 5.00 & -8.4 & 50.0 \\
\hline Carbon tetrachloride & Ave & 0.7217 & 0.7088 & 0.0200 & 4.90 & 5.00 & -1.8 & 50.0 \\
\hline Benzene & Ave & 1.490 & 1.490 & 0.3000 & 5.00 & 5.00 & 0.0 & 25.0 \\
\hline 1,2-Dichloroethane & Ave & 0.2120 & 0.2568 & 0.0100 & 6.10 & 5.00 & 21.1 & 50.0 \\
\hline Trichloroethene & Ave & 0.4234 & 0.3963 & 0.1000 & 4.70 & 5.00 & -6.4 & 25.0 \\
\hline Methylcyclohexane & Ave & 0.5779 & 0.5288 & 0.2000 & 4.60 & 5.00 & -8.5 & 50.0 \\
\hline 1,2-Dichloropropane & Ave & 0.2871 & 0.2911 & 0.1000 & 5.10 & 5.00 & 1.4 & 25.0 \\
\hline Bromodichloromethane & Ave & 0.3586 & 0.3785 & 0.0900 & 5.30 & 5.00 & 5.6 & 25.0 \\
\hline cis-1,3-Dichloropropene & Ave & 0.3705 & 0.3849 & 0.1000 & 5.20 & 5.00 & 3.9 & 25.0 \\
\hline 4-Methyl-2-pentanone & Ave & 0.0652 & 0.0745 & 0.0100 & 57.0 & 50.0 & 14.3 & 50.0 \\
\hline Toluene & Ave & 1.636 & 1.700 & 0.4000 & 5.20 & 5.00 & 3.9 & 25.0 \\
\hline trans-1,3-Dichloropropene & Ave & 0.2641 & 0.2754 & 0.0100 & 5.20 & 5.00 & 4.3 & 25.0 \\
\hline 1,1,2-Trichloroethane & Ave & 0.1475 & 0.1562 & 0.0400 & 5.30 & 5.00 & 5.9 & 25.0 \\
\hline Tetrachloroethene & Ave & 0.4449 & 0.4018 & 0.1000 & 4.50 & 5.00 & -9.7 & 25.0 \\
\hline 2-Hexanone & Ave & 0.0446 & 0.0514 & 0.0100 & 58.0 & 50.0 & 15.3 & 50.0 \\
\hline Dibromochloromethane & Ave & 0.2185 & 0.2334 & 0.0500 & 5.30 & 5.00 & 6.8 & 25.0 \\
\hline 1,2-Dibromoethane & Ave & 0.1292 & 0.1369 & 0.0100 & 5.30 & 5.00 & 6.0 & 25.0 \\
\hline Chlorobenzene & Ave & 1.071 & 1.088 & 0.4000 & 5.10 & 5.00 & 1.6 & 25.0 \\
\hline Ethylbenzene & Ave & 1.886 & 1.950 & 0.5000 & 5.20 & 5.00 & 3.4 & 25.0 \\
\hline
\end{tabular}

FORM VII SOM02.2/VOA_Tr 
FORM VII

GC/MS VOA CONTINUING CALIBRATION DATA

Lab Name: TestAmerica Burlington

SDG No.: 200-38792-1

Lab Sample ID: CCVC 200-117409/23

Instrument ID: CHD.i

GC Column: DB-624

ID $: 0.20(\mathrm{~mm})$

Lab File ID: 25405 23.D

EPA Sample No.: VSTD005EY
Job No.: 200-38792-1

Calibration Date: 06/08/2017 23:42

Calib Start Date: 05/23/2017 17:32

Calib End Date: 05/23/2017 19:16

Conc. Units: ug/L Heated Purge: (Y/N) N

\begin{tabular}{|c|c|c|c|c|c|c|c|c|}
\hline ANALYTE & $\begin{array}{c}\text { CURVE } \\
\text { TYPE }\end{array}$ & AVE RRF & RRF & MIN RRF & $\begin{array}{c}\text { CALC } \\
\text { AMOUNT }\end{array}$ & $\begin{array}{c}\text { SPIKE } \\
\text { AMOUNT }\end{array}$ & $\div D$ & $\begin{array}{l}\mathrm{MAX} \\
\div \mathrm{D}\end{array}$ \\
\hline $\mathrm{m}, \mathrm{p}$-Xylene & Ave & 0.7658 & 0.7993 & 0.2000 & 5.20 & 5.00 & 4.4 & 25.0 \\
\hline o-Xylene & Ave & 0.6733 & 0.7403 & 0.3000 & 5.50 & 5.00 & 9.9 & 25.0 \\
\hline Styrene & Ave & 1.011 & 1.151 & 0.2000 & 5.70 & 5.00 & 13.8 & 25.0 \\
\hline Bromoform & Ave & 0.2328 & 0.2340 & 0.0100 & 5.00 & 5.00 & 0.5 & 50.0 \\
\hline Isopropylbenzene & Ave & 2.023 & 2.161 & 0.7000 & 5.30 & 5.00 & 6.8 & 25.0 \\
\hline $1,1,2,2$-Tetrachloroethane & Ave & 0.1281 & 0.1397 & 0.0500 & 5.40 & 5.00 & 9.0 & 25.0 \\
\hline 1,3-Dichlorobenzene & Ave & 1.695 & 1.567 & 0.5000 & 4.60 & 5.00 & -7.5 & 25.0 \\
\hline 1,4-Dichlorobenzene & Ave & 1.776 & 1.668 & 0.7000 & 4.70 & 5.00 & -6.1 & 25.0 \\
\hline 1,2-Dichlorobenzene & Ave & 1.344 & 1.350 & 0.4000 & 5.00 & 5.00 & 0.5 & 25.0 \\
\hline 1,2-Dibromo-3-chloropropane & Ave & 0.0367 & 0.0368 & 0.0100 & 5.00 & 5.00 & 0.1 & 50.0 \\
\hline 1,2,4-Trichlorobenzene & Ave & 0.7703 & 0.6483 & 0.3000 & 4.20 & 5.00 & $-15 \cdot 8$ & 50.0 \\
\hline 1,2,3-Trichlorobenzene & Ave & 0.5246 & 0.4572 & 0.2000 & 4.40 & 5.00 & -12.8 & 50.0 \\
\hline Vinyl chloride-d3 & Ave & 0.1862 & 0.1644 & 0.0100 & 4.40 & 5.00 & -11.7 & 50.0 \\
\hline Chloroethane-d5 & Ave & 0.1386 & 0.1471 & 0.0100 & 5.30 & 5.00 & 6.2 & 50.0 \\
\hline 1,1-Dichloroethene-d2 & Ave & 0.4559 & 0.4788 & 0.0100 & 5.20 & 5.00 & 5.0 & 25.0 \\
\hline 2-Butanone-d5 & Ave & 0.0228 & 0.0254 & 0.0100 & 56.0 & 50.0 & 11.2 & 50.0 \\
\hline Chloroform-d & Ave & 0.5213 & 0.5885 & 0.0100 & 5.60 & 5.00 & 12.9 & 25.0 \\
\hline 1,2-Dichloroethane-d4 & Ave & 0.1622 & 0.1997 & 0.0100 & 6.20 & 5.00 & 23.1 & 25.0 \\
\hline Benzene-d6 & Ave & 1.454 & 1.465 & 0.0300 & 5.00 & 5.00 & 0.7 & 25.0 \\
\hline 1,2-Dichloropropane-d6 & Ave & 0.3753 & 0.3720 & 0.1000 & 5.00 & 5.00 & -0.9 & 25.0 \\
\hline Toluene-d8 & Ave & 1.411 & 1.456 & 0.2000 & 5.20 & 5.00 & 3.2 & 25.0 \\
\hline trans-1,3-Dichloropropene-d4 & Ave & 0.2267 & 0.2290 & 0.0100 & 5.00 & 5.00 & 1.0 & 25.0 \\
\hline 2-Hexanone-d5 & Ave & 0.0214 & 0.0254 & 0.0100 & 59.0 & 50.0 & 18.5 & 50.0 \\
\hline $1,1,2,2$-Tetrachloroethane-d2 & Ave & 0.1295 & 0.1419 & 0.0100 & 5.50 & 5.00 & 9.6 & 25.0 \\
\hline 1,2-Dichlorobenzene-d4 & Ave & 0.8154 & 0.7848 & 0.0600 & 4.80 & 5.00 & -3.8 & 25.0 \\
\hline
\end{tabular}


FORM I

GC/MS VOA ORGANICS ANALYSIS DATA SHEET

Lab Name: TestAmerica Burlington

Job No : : 200-38792-1

SDG No.: 200-38792-1

Client Sample ID:

Matrix: Water

Analysis Method: SOM02.2/VOA_Tr

Sample wt/vol: 25 (mL)

Soil Aliquot Vol:

Soil Extract Vol.:

․ Moisture:

Analysis Batch No.: 117296

Lab Sample ID: MB 200-117296/7

Lab File ID: 25366_07.D

Date Collected:

Date Analyzed: 06/06/2017 15:43

Dilution Factor: 1

GC Column: DB-624

ID: $0.2(\mathrm{~mm})$

Level: (low/med) Trace

Units: ug/L

\begin{tabular}{|c|c|c|c|c|}
\hline CAS NO. & COMPOUND NAME & RESULT & $Q$ & RL \\
\hline $75-71-8$ & Dichlorodifluoromethane & 0.50 & $\mathrm{U}$ & 0.50 \\
\hline $74-87-3$ & Chloromethane & 0.50 & $\mathrm{U}$ & 0.50 \\
\hline $75-01-4$ & Vinyl chloride & 0.50 & $\mathrm{U}$ & 0.50 \\
\hline $74-83-9$ & Bromomethane & 0.50 & $\mathrm{U}$ & 0.50 \\
\hline $75-00-3$ & Chloroethane & 0.50 & $\mathrm{U}$ & 0.50 \\
\hline $75-69-4$ & Trichlorofluoromethane & 0.50 & $\mathrm{U}$ & 0.50 \\
\hline $75-35-4$ & 1,1-Dichloroethene & 0.50 & $\mathrm{U}$ & 0.50 \\
\hline $76-13-1$ & $\begin{array}{l}\text { 1, 1,2-Trichloro-1,2,2-trifluoroethan } \\
\text { e }\end{array}$ & 0.50 & $\mathrm{U}$ & 0.50 \\
\hline $67-64-1$ & Acetone & 4.8 & $\mathrm{~J}$ & 5.0 \\
\hline $75-15-0$ & Carbon disulfide & 0.14 & $\mathrm{~J}$ & 0.50 \\
\hline $79-20-9$ & Methyl acetate & 0.50 & $\mathrm{U}$ & 0.50 \\
\hline $75-09-2$ & Methylene Chloride & 0.50 & $\mathrm{U}$ & 0.50 \\
\hline $156-60-5$ & trans-1,2-Dichloroethene & 0.50 & $\mathrm{U}$ & 0.50 \\
\hline $1634-04-4$ & Methyl tert-butyl ether & 0.50 & $\mathrm{U}$ & 0.50 \\
\hline $75-34-3$ & 1,1-Dichloroethane & 0.50 & $\mathrm{U}$ & 0.50 \\
\hline $156-59-2$ & cis-1,2-Dichloroethene & 0.50 & $\mathrm{U}$ & 0.50 \\
\hline $78-93-3$ & 2-Butanone & 1.0 & $\mathrm{~J}$ & 5.0 \\
\hline $74-97-5$ & Bromochloromethane & 0.50 & $\mathrm{U}$ & 0.50 \\
\hline $67-66-3$ & Chloroform & 0.50 & $\mathrm{U}$ & 0.50 \\
\hline $71-55-6$ & $1,1,1$-Trichloroethane & 0.50 & $\mathrm{U}$ & 0.50 \\
\hline $110-82-7$ & Cyclohexane & 0.50 & $\mathrm{U}$ & 0.50 \\
\hline $56-23-5$ & Carbon tetrachloride & 0.50 & $\mathrm{U}$ & 0.50 \\
\hline $71-43-2$ & Benzene & 0.50 & $\mathrm{U}$ & 0.50 \\
\hline $107-06-2$ & 1,2-Dichloroethane & 0.50 & $\mathrm{U}$ & 0.50 \\
\hline $79-01-6$ & Trichloroethene & 0.50 & $\mathrm{U}$ & 0.50 \\
\hline $108-87-2$ & Methylcyclohexane & 0.50 & $\mathrm{U}$ & 0.50 \\
\hline $78-87-5$ & 1,2-Dichloropropane & 0.50 & $\mathrm{U}$ & 0.50 \\
\hline $75-27-4$ & Bromodichloromethane & 0.50 & $\mathrm{U}$ & 0.50 \\
\hline $10061-01-5$ & cis-1,3-Dichloropropene & 0.50 & $\mathrm{U}$ & 0.50 \\
\hline $108-10-1$ & 4-Methyl-2-pentanone & 5.0 & $\mathrm{U}$ & 5.0 \\
\hline $108-88-3$ & Toluene & 0.50 & $\mathrm{U}$ & 0.50 \\
\hline $10061-02-6$ & trans-1,3-Dichloropropene & 0.50 & $\mathrm{U}$ & 0.50 \\
\hline $79-00-5$ & 1,1,2-Trichloroethane & 0.50 & $\mathrm{U}$ & 0.50 \\
\hline $127-18-4$ & Tetrachloroethene & 0.50 & $\mathrm{U}$ & 0.50 \\
\hline $591-78-6$ & 2-Hexanone & 5.0 & $\mathrm{U}$ & 5.0 \\
\hline
\end{tabular}

FORM I SOM02.2/VOA_Tr 
FORM I

GC/MS VOA ORGANICS ANALYSIS DATA SHEET

Lab Name: TestAmerica Burlington

SDG No.: 200-38792-1

Client Sample ID:

Matrix: Water

Analysis Method: SOM02.2/VOA_Tr

Sample wt/vol: 25 (mL)

Soil Aliquot Vol:

Soil Extract Vol.:

응 Moisture:

Analysis Batch No.: 117296
Job No.: 200-38792-1

Lab Sample ID: MB 200-117296/7

Lab File ID: 25366_07.D

Date Collected:

Date Analyzed: 06/06/2017 15:43

Dilution Factor: 1

GC Column: DB-624

ID: $0.2(\mathrm{~mm})$

Level: (low/med) Trace

Units: ug/L

\begin{tabular}{|c|c|c|c|c|}
\hline CAS NO. & COMPOUND NAME & RESULT & $Q$ & RL \\
\hline $124-48-1$ & Dibromochloromethane & 0.50 & $\mathrm{U}$ & 0.50 \\
\hline $106-93-4$ & 1,2-Dibromoethane & 0.50 & $\mathrm{U}$ & 0.50 \\
\hline $108-90-7$ & Chlorobenzene & 0.50 & $\mathrm{U}$ & 0.50 \\
\hline $100-41-4$ & Ethylbenzene & 0.50 & $\mathrm{U}$ & 0.50 \\
\hline $95-47-6$ & o-Xylene & 0.50 & $\mathrm{U}$ & 0.50 \\
\hline $179601-23-1$ & m,p-Xylene & 0.50 & $\mathrm{U}$ & 0.50 \\
\hline $100-42-5$ & Styrene & 0.50 & $\mathrm{U}$ & 0.50 \\
\hline $75-25-2$ & Bromoform & 0.50 & $\mathrm{U}$ & 0.50 \\
\hline $98-82-8$ & Isopropylbenzene & 0.50 & $\mathrm{U}$ & 0.50 \\
\hline $79-34-5$ & $1,1,2,2$-Tetrachloroethane & 0.50 & $\mathrm{U}$ & 0.50 \\
\hline $541-73-1$ & 1,3-Dichlorobenzene & 0.50 & $\mathrm{U}$ & 0.50 \\
\hline $106-46-7$ & 1,4-Dichlorobenzene & 0.50 & $\mathrm{U}$ & 0.50 \\
\hline $95-50-1$ & 1,2-Dichlorobenzene & 0.50 & $\mathrm{U}$ & 0.50 \\
\hline $96-12-8$ & 1,2-Dibromo-3-chloropropane & 0.50 & $\mathrm{U}$ & 0.50 \\
\hline $120-82-1$ & $1,2,4$-Trichlorobenzene & 0.16 & $\mathrm{~J}$ & 0.50 \\
\hline $87-61-6$ & $1,2,3$-Trichlorobenzene & 0.29 & $\mathrm{~J}$ & 0.50 \\
\hline
\end{tabular}

\begin{tabular}{|c|c|c|c|c|}
\hline CAS NO. & SURROGATE & $\because \mathrm{REC}$ & $Q$ & LIMITS \\
\hline $6745-35-3$ & Vinyl chloride-d3 & 100 & & $40-130$ \\
\hline $19199-91-8$ & Chloroethane-d5 & 116 & & $65-130$ \\
\hline $22280-73-5$ & 1,1-Dichloroethene-d2 & 93 & & $60-125$ \\
\hline $24313-50-6$ & 2-Butanone-d5 & 129 & & $40-130$ \\
\hline $865-49-6$ & Chloroform-d & 121 & & $70-125$ \\
\hline $17060-07-0$ & 1,2-Dichloroethane-d4 & 136 & * & $70-130$ \\
\hline $1076-43-3$ & Benzene-d6 & 114 & & $70-125$ \\
\hline $93952-08-0$ & 1,2-Dichloropropane-d6 & 93 & & $60-140$ \\
\hline $2037-26-5$ & Toluene-d8 & 113 & & $70-130$ \\
\hline $93951-86-1$ & trans-1,3-Dichloropropene-d4 & 113 & & $55-130$ \\
\hline $4840-82-8$ & 2-Hexanone-d5 & 127 & & $45-130$ \\
\hline $33685-54-0$ & $1,1,2,2$-Tetrachloroethane-d2 & 109 & & $65-120$ \\
\hline $2199-69-1$ & 1,2 -Dichlorobenzene-d4 & 113 & & $80-120$ \\
\hline
\end{tabular}


FORM I

GC/MS VOA ORGANICS ANALYSIS DATA SHEET

TENTATIVELY IDENTIFIED COMPOUNDS

Lab Name: TestAmerica Burlington

SDG No.: 200-38792-1

client Sample ID:

Matrix: Water

Analysis Method: SOM02.2/VOA_Tr

Sample wt/vol: $25(\mathrm{~mL})$

Soil Aliquot Vol:

Soil Extract Vol.:

\% Moisture:

Analysis Batch No.: 117296

Number TICs Found: 1

\begin{tabular}{|c|c|r|r|r|}
\hline CAS NO. & COMPOUND NAME & RT & RESULT & Q \\
QUATCHTY \\
\hline \hline
\end{tabular}

Job No.: 200-38792-1

Lab Sample ID: MB 200-117296/7

Lab File ID: 25366_07.D

Date Collected:

Date Analyzed: 06/06/2017 15:43

Dilution Factor: 1

GC Column: DB-624

ID: $0.2(\mathrm{~mm})$

Level: (low/med) Trace

Units: ug/L

TIC Result Total: 3.1 
FORM I

GC/MS VOA ORGANICS ANALYSIS DATA SHEET

TARGETED TENATIVELY IDENTIFIED COMPOUNDS

Lab Name: TestAmerica Burlington

SDG No.: 200-38792-1

client Sample ID:

Matrix: Water

Analysis Method: SOM02.2/VOA_Tr

Sample wt/vol: 25 (mL)

Soil Aliquot Vol:

Soil Extract Vol.:

․ Moisture:

Analysis Batch No.: 117296
Job No.: 200-38792-1

Lab Sample ID: MB 200-117296/7

Lab File ID: 25366 07.D

Date Collected:

Date Analyzed: 06/06/2017 15:43

Dilution Factor: 1

GC Column: DB-624

ID: $0.2(\mathrm{~mm})$

Level: (low/med) Trace

Units: ug/L

\begin{tabular}{|c|c|c|c|c|}
\hline CAS NO. & COMPOUND NAME & RT & RESULT & Q \\
\hline QUALITY
\end{tabular}


FORM I

GC/MS VOA ORGANICS ANALYSIS DATA SHEET

Lab Name: TestAmerica Burlington

Job No : : 200-38792-1

SDG No.: 200-38792-1

Client Sample ID:

Matrix: Water

Analysis Method: SOM02.2/VOA_Tr

Sample wt/vol: 25 (mL)

Soil Aliquot Vol:

Soil Extract Vol.:

․ Moisture:

Analysis Batch No.: 117409

Lab Sample ID: MB 200-117409/6

Lab File ID: 25405_06.D

Date Collected:

Date Analyzed: 06/08/2017 16:19

Dilution Factor: 1

GC Column: DB-624

ID: $0.2(\mathrm{~mm})$

Level: (low/med) Trace

Units: ug/L

\begin{tabular}{|c|c|c|c|c|}
\hline CAS NO. & COMPOUND NAME & RESULT & $Q$ & RL \\
\hline $75-71-8$ & Dichlorodifluoromethane & 0.50 & $\mathrm{U}$ & 0.50 \\
\hline $74-87-3$ & Chloromethane & 0.50 & $\mathrm{U}$ & 0.50 \\
\hline $75-01-4$ & Vinyl chloride & 0.50 & $\mathrm{U}$ & 0.50 \\
\hline $74-83-9$ & Bromomethane & 0.13 & $\mathrm{~J}$ & 0.50 \\
\hline $75-00-3$ & Chloroethane & 0.50 & $\mathrm{U}$ & 0.50 \\
\hline $75-69-4$ & Trichlorofluoromethane & 0.50 & $\mathrm{U}$ & 0.50 \\
\hline $75-35-4$ & 1,1-Dichloroethene & 0.50 & $\mathrm{U}$ & 0.50 \\
\hline $76-13-1$ & $\begin{array}{l}\text { 1, 1,2-Trichloro-1,2,2-trifluoroethan } \\
\text { e }\end{array}$ & 0.50 & $\mathrm{U}$ & 0.50 \\
\hline $67-64-1$ & Acetone & 2.5 & $\mathrm{~J}$ & 5.0 \\
\hline $75-15-0$ & Carbon disulfide & 0.13 & $\mathrm{~J}$ & 0.50 \\
\hline $79-20-9$ & Methyl acetate & 0.50 & $\mathrm{U}$ & 0.50 \\
\hline $75-09-2$ & Methylene Chloride & 0.50 & $\mathrm{U}$ & 0.50 \\
\hline $156-60-5$ & trans-1,2-Dichloroethene & 0.50 & $\mathrm{U}$ & 0.50 \\
\hline $1634-04-4$ & Methyl tert-butyl ether & 0.50 & $\mathrm{U}$ & 0.50 \\
\hline $75-34-3$ & 1,1-Dichloroethane & 0.50 & $\mathrm{U}$ & 0.50 \\
\hline $156-59-2$ & cis-1,2-Dichloroethene & 0.50 & $\mathrm{U}$ & 0.50 \\
\hline $78-93-3$ & 2-Butanone & 5.0 & $\mathrm{U}$ & 5.0 \\
\hline $74-97-5$ & Bromochloromethane & 0.50 & $\mathrm{U}$ & 0.50 \\
\hline $67-66-3$ & Chloroform & 0.50 & $\mathrm{U}$ & 0.50 \\
\hline $71-55-6$ & $1,1,1$-Trichloroethane & 0.50 & $\mathrm{U}$ & 0.50 \\
\hline $110-82-7$ & Cyclohexane & 0.50 & $\mathrm{U}$ & 0.50 \\
\hline $56-23-5$ & Carbon tetrachloride & 0.50 & $\mathrm{U}$ & 0.50 \\
\hline $71-43-2$ & Benzene & 0.50 & $\mathrm{U}$ & 0.50 \\
\hline $107-06-2$ & 1,2-Dichloroethane & 0.50 & $\mathrm{U}$ & 0.50 \\
\hline $79-01-6$ & Trichloroethene & 0.50 & $\mathrm{U}$ & 0.50 \\
\hline $108-87-2$ & Methylcyclohexane & 0.50 & $\mathrm{U}$ & 0.50 \\
\hline $78-87-5$ & 1,2-Dichloropropane & 0.50 & $\mathrm{U}$ & 0.50 \\
\hline $75-27-4$ & Bromodichloromethane & 0.50 & $\mathrm{U}$ & 0.50 \\
\hline $10061-01-5$ & cis-1,3-Dichloropropene & 0.50 & $\mathrm{U}$ & 0.50 \\
\hline $108-10-1$ & 4-Methyl-2-pentanone & 5.0 & $\mathrm{U}$ & 5.0 \\
\hline $108-88-3$ & Toluene & 0.50 & $\mathrm{U}$ & 0.50 \\
\hline $10061-02-6$ & trans-1,3-Dichloropropene & 0.50 & $\mathrm{U}$ & 0.50 \\
\hline $79-00-5$ & 1,1,2-Trichloroethane & 0.50 & $\mathrm{U}$ & 0.50 \\
\hline $127-18-4$ & Tetrachloroethene & 0.50 & $\mathrm{U}$ & 0.50 \\
\hline $591-78-6$ & 2-Hexanone & 5.0 & $\mathrm{U}$ & 5.0 \\
\hline
\end{tabular}

FORM I SOM02.2/VOA_Tr 
FORM I

GC/MS VOA ORGANICS ANALYSIS DATA SHEET

Lab Name: TestAmerica Burlington

SDG No.: 200-38792-1

Client Sample ID:

Matrix: Water

Analysis Method: SOM02.2/VOA_Tr

Sample wt/vol: 25 (mL)

Soil Aliquot Vol:

Soil Extract Vol.:

응 Moisture:

Analysis Batch No.: 117409
Job No. : 200-38792-1

Lab Sample ID: MB 200-117409/6

Lab File ID: 25405_06.D

Date Collected:

Date Analyzed: 06/08/2017 16:19

Dilution Factor: 1

GC Column: DB-624

ID: $0.2(\mathrm{~mm})$

Level: (low/med) Trace

Units: ug/L

\begin{tabular}{|c|c|c|c|c|}
\hline CAS NO. & COMPOUND NAME & RESULT & $Q$ & RL \\
\hline $124-48-1$ & Dibromochloromethane & 0.50 & $\mathrm{U}$ & 0.50 \\
\hline $106-93-4$ & 1,2-Dibromoethane & 0.50 & $\mathrm{U}$ & 0.50 \\
\hline $108-90-7$ & Chlorobenzene & 0.50 & $\mathrm{U}$ & 0.50 \\
\hline $100-41-4$ & Ethylbenzene & 0.50 & $\mathrm{U}$ & 0.50 \\
\hline $95-47-6$ & o-Xylene & 0.50 & $\mathrm{U}$ & 0.50 \\
\hline $179601-23-1$ & m,p-Xylene & 0.50 & $\mathrm{U}$ & 0.50 \\
\hline $100-42-5$ & Styrene & 0.50 & $\mathrm{U}$ & 0.50 \\
\hline $75-25-2$ & Bromoform & 0.50 & $\mathrm{U}$ & 0.50 \\
\hline $98-82-8$ & Isopropylbenzene & 0.50 & $\mathrm{U}$ & 0.50 \\
\hline $79-34-5$ & $1,1,2,2$-Tetrachloroethane & 0.50 & $\mathrm{U}$ & 0.50 \\
\hline $541-73-1$ & 1,3-Dichlorobenzene & 0.50 & $\mathrm{U}$ & 0.50 \\
\hline $106-46-7$ & 1,4-Dichlorobenzene & 0.078 & $\mathrm{~J}$ & 0.50 \\
\hline $95-50-1$ & 1,2-Dichlorobenzene & 0.50 & $\mathrm{U}$ & 0.50 \\
\hline $96-12-8$ & 1,2-Dibromo-3-chloropropane & 0.50 & $\mathrm{U}$ & 0.50 \\
\hline $120-82-1$ & $1,2,4$-Trichlorobenzene & 0.30 & $\mathrm{~J}$ & 0.50 \\
\hline $87-61-6$ & $1,2,3$-Trichlorobenzene & 0.48 & $\mathrm{~J}$ & 0.50 \\
\hline
\end{tabular}

\begin{tabular}{|c|c|c|c|c|}
\hline CAS NO. & SURROGATE & $\because \mathrm{REC}$ & $Q$ & LIMITS \\
\hline $6745-35-3$ & Vinyl chloride-d3 & 95 & & $40-130$ \\
\hline $19199-91-8$ & Chloroethane-d5 & 100 & & $65-130$ \\
\hline $22280-73-5$ & 1,1-Dichloroethene-d2 & 75 & & $60-125$ \\
\hline $24313-50-6$ & 2-Butanone-d5 & 113 & & $40-130$ \\
\hline $865-49-6$ & Chloroform-d & 100 & & $70-125$ \\
\hline $17060-07-0$ & 1,2-Dichloroethane-d4 & 107 & & $70-130$ \\
\hline $1076-43-3$ & Benzene-d6 & 107 & & $70-125$ \\
\hline $93952-08-0$ & 1,2-Dichloropropane-d6 & 87 & & $60-140$ \\
\hline $2037-26-5$ & Toluene-d8 & 108 & & $70-130$ \\
\hline $93951-86-1$ & trans-1,3-Dichloropropene-d4 & 112 & & $55-130$ \\
\hline $4840-82-8$ & 2-Hexanone-d5 & 136 & * & $45-130$ \\
\hline $33685-54-0$ & $1,1,2,2$-Tetrachloroethane-d2 & 101 & & $65-120$ \\
\hline $2199-69-1$ & 1,2-Dichlorobenzene-d4 & 108 & & $80-120$ \\
\hline
\end{tabular}


FORM I

GC/MS VOA ORGANICS ANALYSIS DATA SHEET

TENTATIVELY IDENTIFIED COMPOUNDS

Lab Name: TestAmerica Burlington

SDG No.: 200-38792-1

client Sample ID:

Matrix: Water

Analysis Method: SOM02.2/VOA_Tr

Sample wt/vol: 25 (mL)

Soil Aliquot Vol:

Soil Extract Vol.:

\% Moisture:

Analysis Batch No.: 117409

Number TICs Found: 1

\begin{tabular}{|c|c|c|c|c|}
\hline CAS NO. & COMPOUND NAME & RT & RESULT & Q \\
QUATCHTY \\
\hline \hline
\end{tabular}

Job No.: 200-38792-1

Lab Sample ID: MB 200-117409/6

Lab File ID: 25405_06.D

Date Collected:

Date Analyzed: 06/08/2017 16:19

Dilution Factor: 1

GC Column: DB-624

ID: $0.2(\mathrm{~mm})$

Level: (low/med) Trace

Units: ug/L

TIC Result Total: 3.1 
FORM I

GC/MS VOA ORGANICS ANALYSIS DATA SHEET

TARGETED TENATIVELY IDENTIFIED COMPOUNDS

Lab Name: TestAmerica Burlington

SDG No.: 200-38792-1

client Sample ID:

Matrix: Water

Analysis Method: SOM02.2/VOA_Tr

Sample wt/vol: 25 (mL)

Soil Aliquot Vol:

Soil Extract Vol.:

․ Moisture:

Analysis Batch No.: 117409
Job No.: 200-38792-1

Lab Sample ID: MB 200-117409/6

Lab File ID: 25405 06.D

Date Collected:

Date Analyzed: 06/08/2017 16:19

Dilution Factor: 1

GC Column: DB-624

ID: $0.2(\mathrm{~mm})$

Level: (low/med) Trace

Units: ug/L

\begin{tabular}{|c|c|c|c|c|}
\hline CAS NO. & COMPOUND NAME & RT & RESULT & Q \\
\hline QUALITY
\end{tabular}


GC/MS VOA ANALYSIS RUN LOG

Lab Name: TestAmerica Burlington

SDG No.: 200-38792-1

Instrument ID: CHD.i

Analysis Batch Number: 116931
Job No.: 200-38792-1

Start Date: 05/23/2017 13:58

End Date: 05/23/2017 19:42

\begin{tabular}{|c|c|c|c|c|c|c|}
\hline LAB SAMPLE ID & CLIENT SAMPLE ID & DATE ANA & $Y Z E D$ & DILUTION & LAB FILE ID & COLUMN ID \\
\hline ZZzZZ & & $05 / 23 / 2017$ & $13: 58$ & 1 & & DB-624 $0.2(\mathrm{~mm})$ \\
\hline ZZzZZ & & $05 / 23 / 2017$ & $14: 23$ & 1 & & DB-624 $0.2(\mathrm{~mm})$ \\
\hline ZZZZZ & & $05 / 23 / 2017$ & $14: 49$ & 1 & & DB-624 $0.2(\mathrm{~mm})$ \\
\hline BFB 200-116931/4 & & $05 / 23 / 2017$ & $16: 12$ & 1 & 25203_04.D & $\mathrm{DB}-6240.2(\mathrm{~mm})$ \\
\hline ZZZZZ & & $05 / 23 / 2017$ & $16: 41$ & 1 & & $\mathrm{DB}-6240.2(\mathrm{~mm})$ \\
\hline ZZZZZ & & $05 / 23 / 2017$ & $17: 06$ & 1 & & $\mathrm{DB}-624 \quad 0.2(\mathrm{~mm})$ \\
\hline IC $200-116931 / 26$ & & $05 / 23 / 2017$ & $17: 32$ & 1 & 25203_07.D & $\mathrm{DB}-6240.2(\mathrm{~mm})$ \\
\hline IC 200-116931/27 & & $05 / 23 / 2017$ & $17: 58$ & 1 & 25203_08.D & DB-624 $0.2(\mathrm{~mm})$ \\
\hline ICIS $200-116931 / 28$ & & $05 / 23 / 2017$ & $18: 24$ & 1 & 25203_09.D & DB-624 $0.2(\mathrm{~mm})$ \\
\hline IC $200-116931 / 29$ & & $05 / 23 / 2017$ & $18: 50$ & 1 & 25203_10.D & $\mathrm{DB}-6240.2(\mathrm{~mm})$ \\
\hline IC $200-116931 / 30$ & & $05 / 23 / 2017$ & $19: 16$ & 1 & 25203_11.D & $\mathrm{DB}-6240.2(\mathrm{~mm})$ \\
\hline ICV 200-116931/31 & & $05 / 23 / 2017$ & $19: 42$ & 1 & 25203_12.D & $\mathrm{DB}-624 \quad 0.2(\mathrm{~mm})$ \\
\hline
\end{tabular}


GC/MS VOA ANALYSIS RUN LOG

Lab Name: TestAmerica Burlington

SDG No.: 200-38792-1

Instrument ID: CHD.i

Analysis Batch Number: 117296
Job No.: 200-38792-1

Start Date: 06/06/2017 13:46

End Date: 06/07/2017 00:28

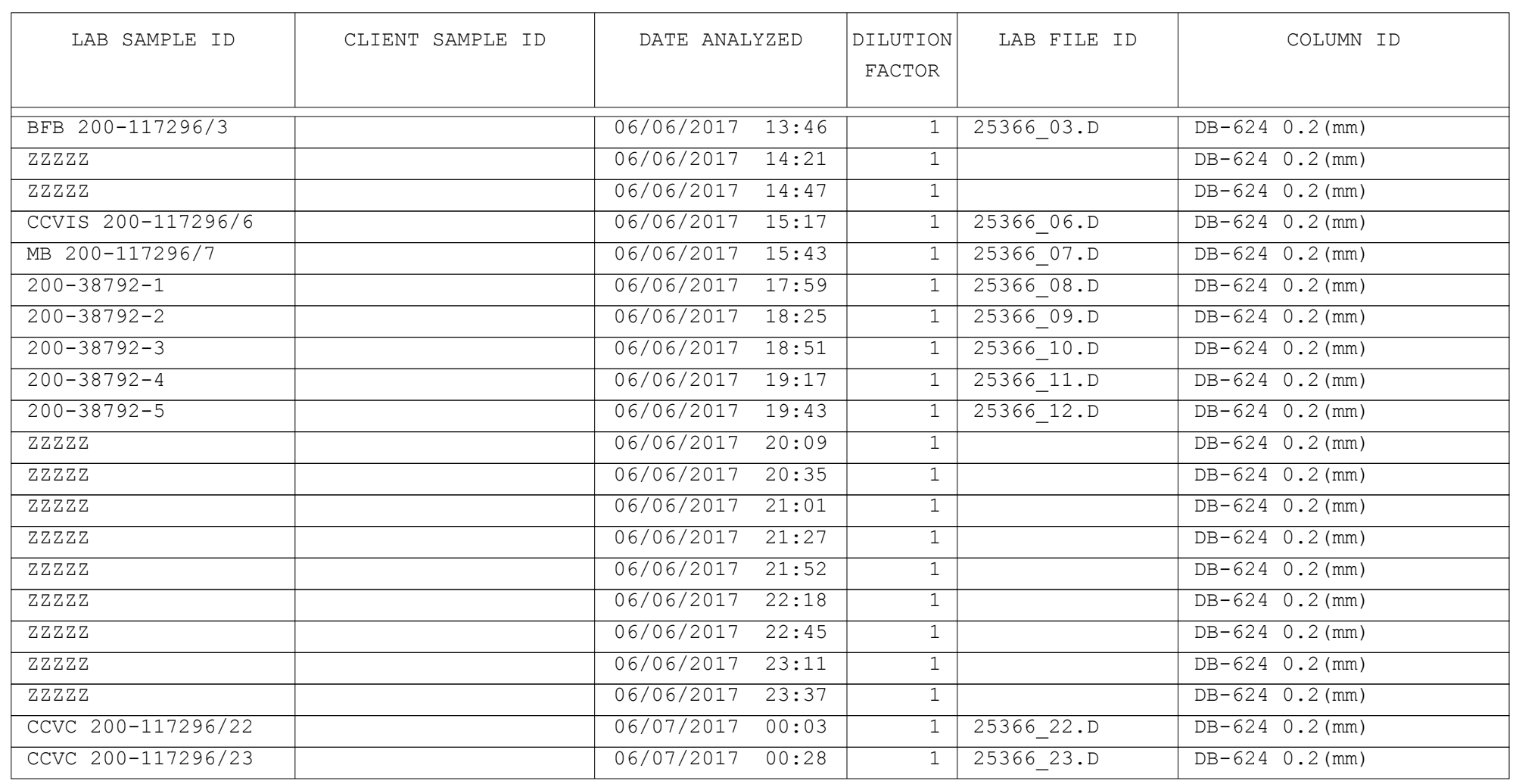


GC/MS VOA ANALYSIS RUN LOG

Lab Name: TestAmerica Burlington

SDG No.: 200-38792-1

Instrument ID: CHD.i

Analysis Batch Number: 117409
Job No.: 200-38792-1

Start Date: 06/08/2017 14:03

End Date: 06/08/2017 23:42

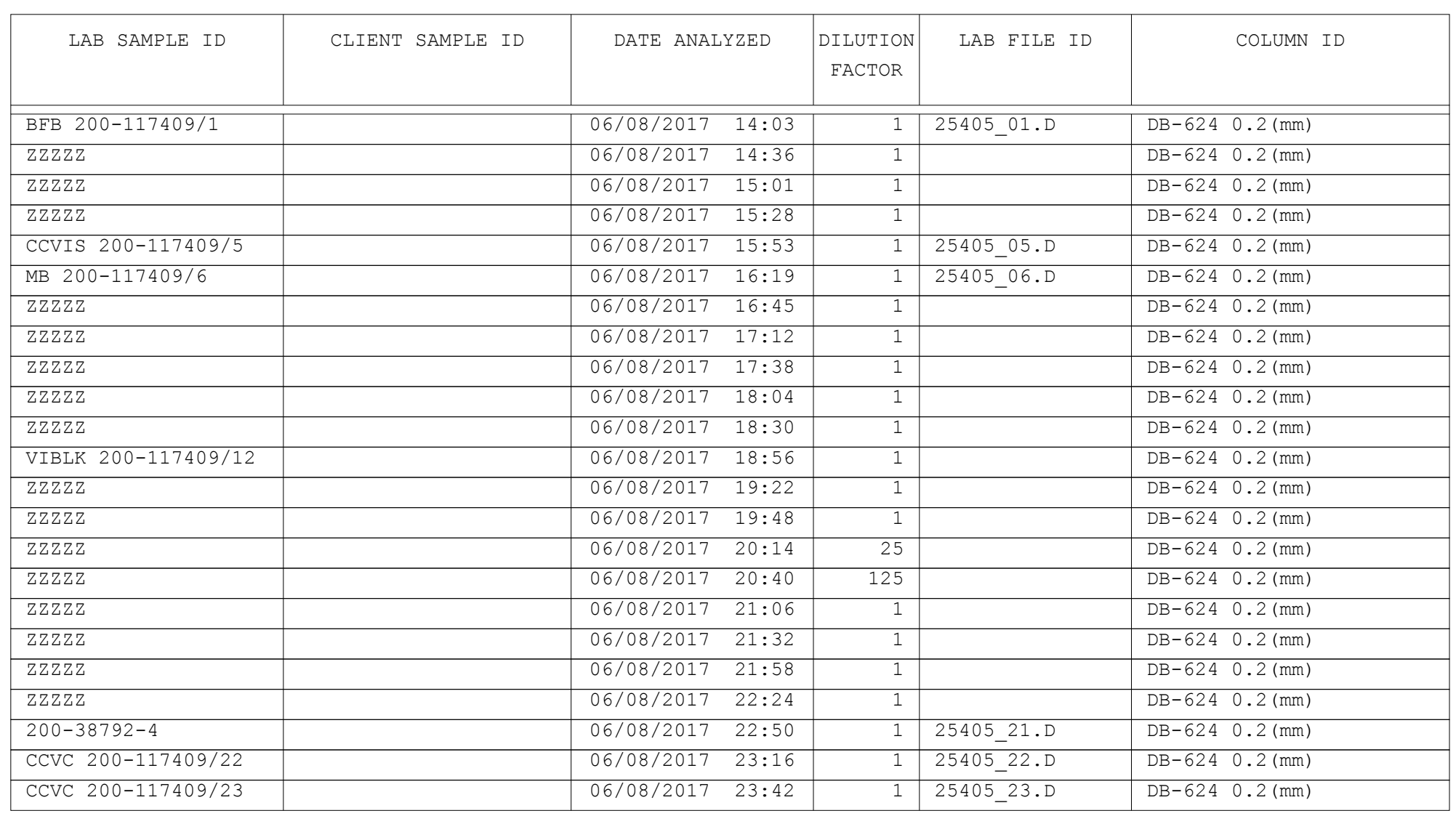




\section{Shipping and Receiving Documents}




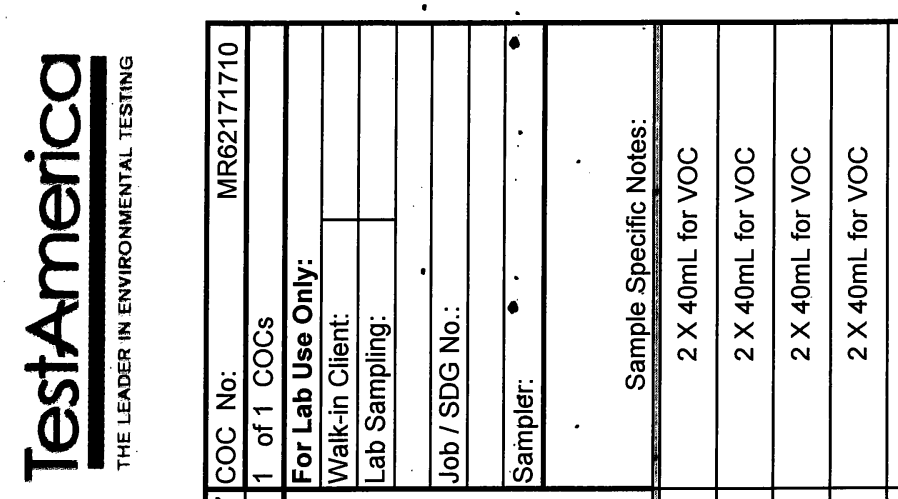




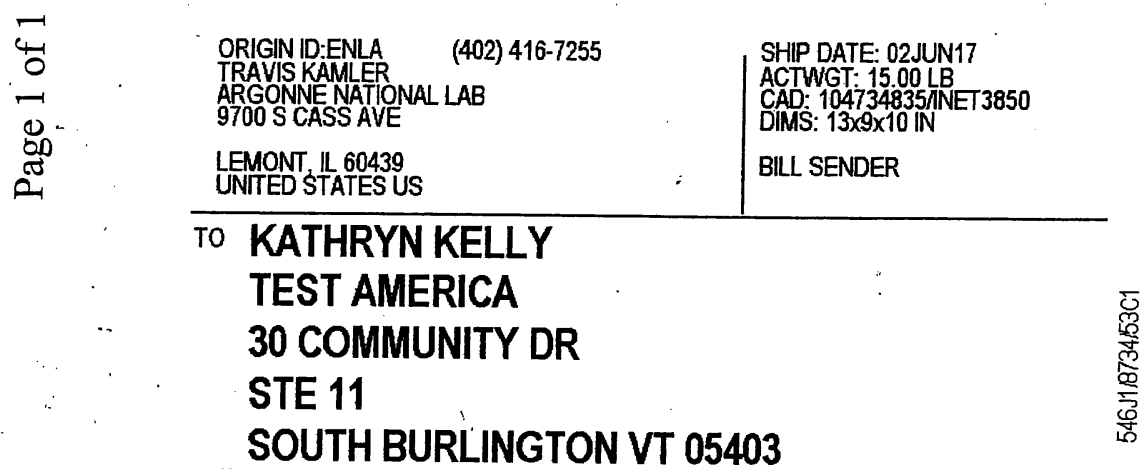

(802) 660-1990
RV:
PO: MORRLLL WATERS

|| ||||||||||||||||||||||||||||||||||||||||||||||||||||||||||
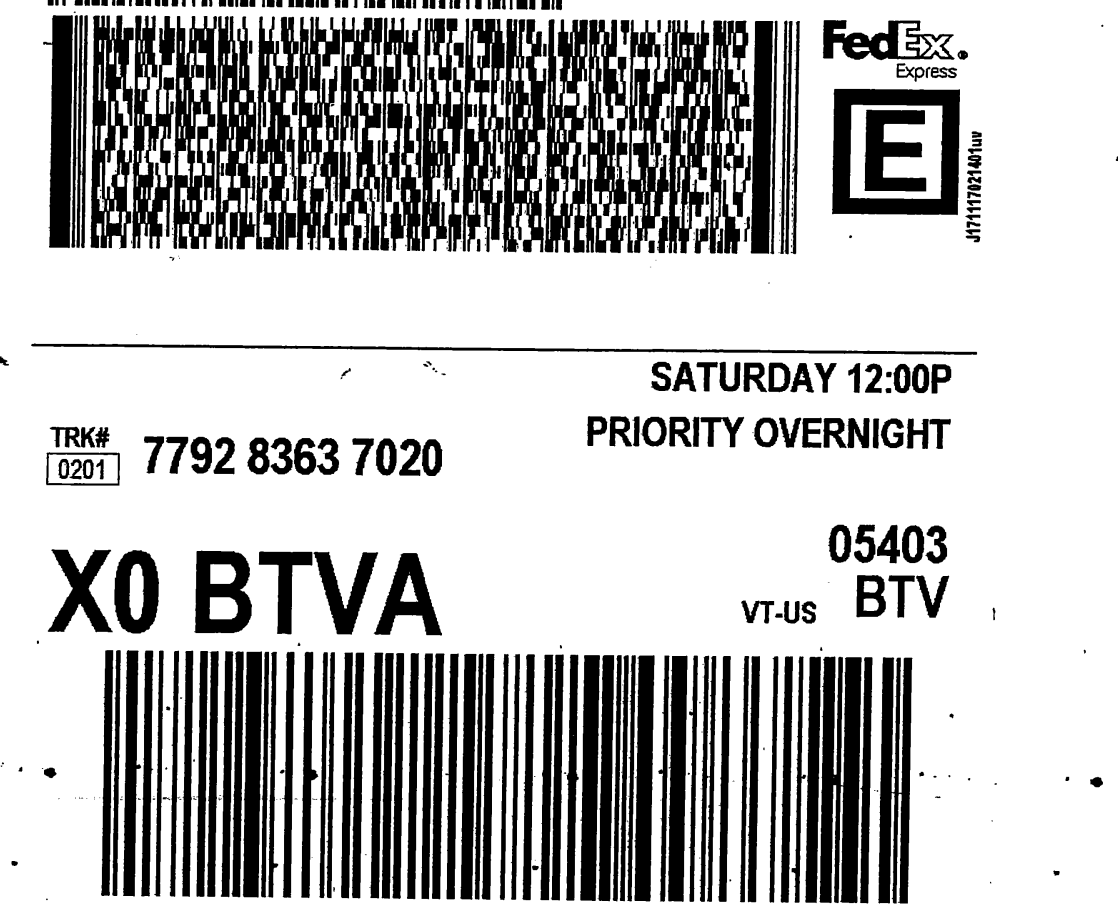


\section{Login Sample Receipt Checklist}

Client: Argonne National Laboratory

Job Number: 200-38792-1

SDG Number: 200-38792-1

Login Number: 38792

List Source: TestAmerica Burlington

List Number: 1

Creator: Johnson, Eleanor E

Question

Radioactivity wasn't checked or is $</=$ background as measured by a survey True meter.

The cooler's custody seal, if present, is intact.

Sample custody seals, if present, are intact.

The cooler or samples do not appear to have been compromised or tampered with.

Samples were received on ice.

Cooler Temperature is acceptable.

Cooler Temperature is recorded.

$\mathrm{COC}$ is present.

COC is filled out in ink and legible.

$\mathrm{COC}$ is filled out with all pertinent information.

Is the Field Sampler's name present on COC?

There are no discrepancies between the containers received and the COC.

Samples are received within Holding Time (excluding tests with immediate

HTs)

Sample containers have legible labels.

Containers are not broken or leaking.

Sample collection date/times are provided.

Appropriate sample containers are used.

Sample bottles are completely filled.

Sample Preservation Verified.

There is sufficient vol. for all requested analyses, incl. any requested MS/MSDs

Containers requiring zero headspace have no headspace or bubble is $<6 \mathrm{~mm}$ (1/4").

Multiphasic samples are not present.

Samples do not require splitting or compositing.

Residual Chlorine Checked.

Answer Comment

True

Lab does not accept radioactive samples.

Seal present with no number.

True

True

True

True

True

True

True

True

False

True

True

True

True

True

True

True

True

True

True

True

True

N/A
No analysis requiring residual chlorine check assigned. 


\section{AGEM Team at Argonne}

Lorraine M. LaFreniere, Ph.D.

Principal Geological Engineer

Program Manager

Jorge S. Alvarado, Ph.D.

Chemist

Laboratory Manager and Analyst

Esther E. Bowen, M.S.

Quality Assurance/Quality Control and Records

Data Management Specialist

Manager

Laurel A. Culbert, B.A.

Graphic Artist/Designer

Report Graphics and Maps (including GIS)

James Hansen, B.A.

Program Coordinator/Manager

Site Reconnaissance and Community Relations

Louis E. Martino, M.S.

Environmental Systems Engineer/Program

Manager

Technical Lead/Remediation Specialist (Cost Engineering)

Terri Patton, M.S.

Principal Geologist

Technical Editor

Candace M. Rose, B.S.

Scientific Associate Senior

Laboratory Analyst

Robert A. Sedivy, M.S.

Hydrogeologist

Technical Lead/Hydrogeologist

Eugene Y. Yan, Ph.D.

Earth Scientist

Technical Lead/Hydrogeologist 
Argonne

Environmental Science Division

Argonne National Laboratory

9700 South Cass Avenue, BIdg. 203

Argonne, IL 60439-4843

www.anl.gov 